\title{
Contribution to the knowledge of the Curculionoidea fauna of the Danube Delta and the Dobruja Region (Romania)
}

\author{
Irén Kocs, Jiří KrátKÝ, Attila Podlussány \& Lucian Alexandru TeOdoR
}

\begin{abstract}
Summary: Presently 724 species are known from the Danube Delta and Dobruja. From these, 270 in the Danube Delta and Dobruja, 50 to Romania and 2 to science are new (the description of the species is in progress). The species new to Romania are the following: Noxius curtirostris (Mulsant \& ReY, 1861); Bruchela cana (KüSTER, 1848); B. concolor (FÅHRAEus, 1839); B. parvula (Motschulsky, 1875); Perapion (Perapion) connexum (SCHILSKY, 1902); Ceratapion (Echinostroma) scalptum caviceps (DeSBrochers DES Loges, 1870); Metapion ermischi (Voss, 1969); Protapion angusticolle (Gyllenhal, 1833); Allomalia quadrivirgata (Costa, 1863); Corimalia fausti fausti (ReITter, 1890); Bagous (Macropelmus) claudicans BoHEMAN, 1845; B. (Macropelmus) dieckmanni Gratshev, 1993; B. (Macropelmus) lothari Caldara \& O’Brien, 1998; Brachycerus lutosus Gyllenhal, 1833; Stenopelmus rufinasus Gyllenhal, 1835; Tanysphyrus ater Blatchley, 1928; Aulacobaris kiesenwetteri (Faust, 1890); Labiaticola despicatus Faust, 1889; L. melas (Boheman, 1836); Ceutorhynchus levantinus Schultze, 1898; C. merkli Korotyaev, 2001; C. scrobicollis Neresheimer \& H. Wagner, 1924; C. talickyi Korotyaev, 1980; C. varius Rey, 1895; Microplontus millefolii (Schultze, 1897); Ranunculiphilus (Austroceutorhynchus) italicus (C. N. F. BRIsout De BARNeville, 1869); Thamiocolus roubali DiecKMANN, 1973; T. sinapis (Desbrochers des Loges, 1893); Gymnetron tibiellum Desbrochers Des Loges, 1900; Miarus hellenicus DieckmanN 1978; M. solarii SmreczyŃski, 1957; Philernus ponticus Korotyaev, 1979; Sibinia (Sibinia) femoralis Germar, 1823; S. (Sibinia) variata Gyllenhal, 1835; Tychius (Tychius) ochraceus Tournier, 1873; T. (Tychius) subsulcatus Tournier, 1874; Ptochus (Ptochus) porcellus Boheman, 1834; Omias murinus (Boheman, 1842); Otiorhynchus (Pseudocryphiphorus) zebei STIERLIN, 1861; Argoptochus (Argoptochus) markovensis Angelov, 1987; Polydrusus (Conocetus) baudii (FAust, 1889); Sitona ophthalmicus DeSBROCHERS DES Loges, 1869; Chlorophanus viridis balcanicus BeHNe, 1989; Rhabdorrhynchus echii BrAHM, 1790; Bangasternus planifrons (Brullé, 1832); Larinus (Larinomesius) atomarius CAPIOMONT, 1874; L. (Larinomesius) canescens GyllenHAL, $1835 ;$ L. (Larinomesius) syriacus Gyllenhal, 1835; L. (Phyllonomeus) filiformis Petri, 1907; Lixus (Epimeces) scolopax Boheman, 1835.
\end{abstract}

Key words: Curculionoidea, faunistic, new species, Danube Delta, Dobruja, Romania

\section{Introduction}

The Danube Delta is the second largest river delta in Europe, after the Volga Delta. It is bordered south-west by the highlands of Dobruja and by the Romanian-Ukainian border in the North. The Danube river flows into the Black Sea in the East. The Delta is part of the World Heritage since 1991. Dobruja is a historical region on the Balkan Peninsula. Today most of it belongs to Romania and a small part to Bulgaria. The Romanian part consists of two counties: Tulcea and Constanţa. It is bordered by the Danube river in the North, by Bulgaria in the South, and in the East by the Black Sea (https://hu.wikipedia.org/wiki/ Dobrudzsa).

The World Heritage Danube Delta and Dobruja with their spectacular Black Sea elements, rare species, western and mediterranean components, project irresistable attractiveness to naturalists. Before human interaction this area was a huge, forest steppe with lakes and lagunes. In some parts conspicuous rocks are found. The most typical examples can be found in the Măcin Mountains with their enormous and oddly shaped rocks. Other spectacular landscapes can be found at the Dobruja Canyon and the Gura Dobrogei, Canaraura Fetii and Hârşova rocky environments. Presently however this area is nothing else but an enormous agricultural land where nature is more or less protected in some smaller nature reserves, national parks and Natura 2000 areas. An acknowledgement and clearly defined boundaries of these areas are lacking. However, the future of biodiversity in the Măcin Mountains National Parks, is relatively secured because of the difficult access, the large forests and the lack of destructive mass tourism (Lengyel http://regithink.transindex.ro). However, care must be taken that in the future the area will not be affected by illegal deforestation, as is unfortunately the case in other areas of Romania. Intensive grazing can also be a threat to this area.

In this work, apart of the data from the literature, we are publishing the data of Curculionoidea in the collections of the Complexul Muzeal de Ştiinţele Naturii "Răsvan Angheluţă" Galaţi of Galaţi, the Muzeul Naţional de Istorie Naturală "Grigore Antipa" Bucureşti of Bucureşti and the Magyar 
Természettudományi Múzeum of Budapest and the processed material of numerous entomological collecting trips in the area by 26 entomologists.

\section{Material and methods}

The first published data from the literature originate from the end of the 19th Century. Several notable entomologists, such as Arnold Lucien MonTANDON, Maurice JAQUET collected in the Danube Delta and in Dobruja. The literature mentions 397 species from the Danube Delta and Dobruja, from which 8 are puted by us in square brackets, as they do not live here and it is presumed they were included only because of erroneous determination.

The Galaţi and the Bukarest museums' materials were determined by Irén Kocs and Attila PoDLussánY. In the material from Galaţi 59 and in the collection from Bukarest 258 species were confirmed. In the material of the Magyar Természettudományi Múzeum (Budapest) Attila Podlussány and Edmund ReITter confirmed 5 species. In the species list they indicated the collection data (locality, date, name) and the position of the specimens.

The material collected by the entomologists originates from 75 localities (fig. 1). These were

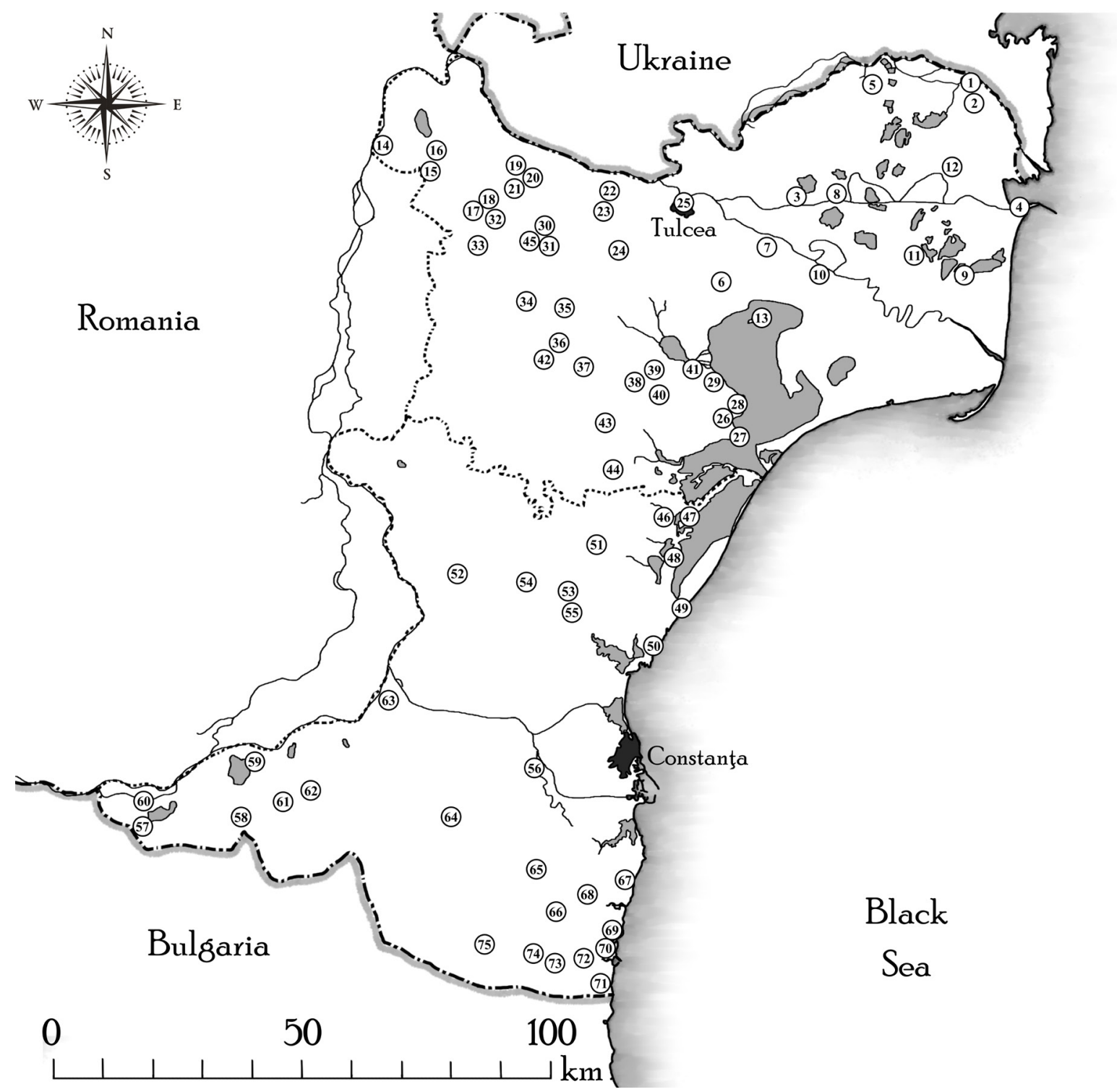

Fig. 1. The most important collecting sites of the Danube Delta and the Dobrogea Region

Tulcea County: 1 Periprava; 2 Pădurea Letea; 3 Maliuc, Mila 26; 4 Sulina; 5 Canalul Olguţa; 6 Agighiol; 7 Beştepe; 8 Gorgova; 9 Lacul Roşu; 10 Murighiol (Braţul Sfântu Gheorghe); 11 Caraorman; 12 Canalul Magearu; 13 Insula Popina (Rezervaţia faunistică Insula Popina); 14 Smârdan; 15 Măcin; 16 Jijila; 17 Greci; 18 Vârful Țuţuiatul, Munţii Măcin; 19 Luncaviţa; 20 Pricopan (Culmea Pricopanului); 21 Valea Fagilor; 22 Parcheş; 23 Somova; 24 Frecăţei; 25 Tulcea; 26 Jurilovaca, Sãlcioara; 27 Doloşman; 28 Iancina; 29 Dealul Taşburun; 30 Nifon; 31 Hamcearca; 32 Creasta Cardonului, Munţii Măcin; 33 Turcoaia; 34 General Praporgescu; 35 Horia; 36 Atmagea; 37 Ciucurova; 38 Slava Rusă; 39 Babadag; 40 Codru Monastery; 41 Enisala; 42 Topolog; 43 Stejaru; 44 Beidaud; 45 Hamcearca; Constanţa County: 46 Sinoie; 47 Sinoie, Grindul Lupilor; 48 Istria, Cetatea Histria; 49 Vadu, Grindul Chituc; 50 Corbu; 51 Tariverde; 52 Crucea; 53 Târguşor, Gura Dobrogei; 54 Cheia, Cheile Dobrogei; 55 Palazu Mic; 56 Murfatlar, Fântâniţa; 57 Pădurea Esechioi; 58 Canaraua Fetii; 59 Oltina; 60 Ostrov, Bugeac; 61 Negureni; 62 Ion Corvin; 63 Cochirleni; 64 Cobadin; 65 Amzacea; 66 Pecineaga; 67 Costineşti; 6823 August; 69 NeptunOlimp; 70 Mangalia, Saturn; 71 Vama Veche; 72 Balta Limanu; 73 Pădurea Hagieni; 74 Albeşti; 75 Negru Vodă. 
visited on several occasions in spring, summer and autumn. The collections were carried out by vegetation netting and beating, leaf litter sifting, soil and light trapping and single captures under stones. The authors identified 609 species.

For identification to species level different keys for weevil determination were used: ENDRőDI S. 1960, 1961, 1963, 1968, 1971; ANGelov 1976; AlonsoZarazaga 1989, 1990; Borovec 1992; Borovec and Pelletier 2009; Skuhrovec 2009; Skuhrovec et al. 2010, 2014; STÜBEN et al. 2011, 2013, 2014, 2015).

\section{The coordinates of the habitat:}

Tulcea County:

- Periprava (meadows), 45²4'28.45' N, 29³2'35.82'"E, 2 m;

Pădurea Letea (forest) $45^{\circ} 22^{\prime} 11.45^{\prime} \mathrm{N}$, 2932'53.47' E, 0 m;

- Maliuc, Mila 26, $45^{\circ} 11^{\prime} 02.55^{\prime \prime N}, 29^{\circ} 06^{\circ} 06.34^{\prime \prime} \mathrm{E}, 2 \mathrm{~m}$;

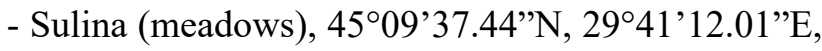
$1 \mathrm{~m}$;

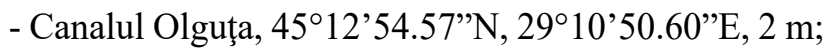
- Agighiol (reed areas and meadows with shrubs and

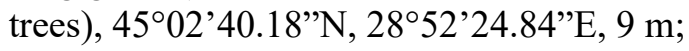

- Beştepe (meadows with shrubs), $44^{\circ} 05^{\prime} 31.42^{\prime} \mathrm{N}$, 2902'22.46'"E, $162 \mathrm{~m}$;

- Gorgova (meadows with shrubs), $45^{\circ} 11^{\prime} 05.10^{\prime \prime} \mathrm{N}$, $29^{\circ} 12^{\prime} 06.23$ 'E, 2 m;

- Caraorman, (meadows and forest) $45^{\circ} 04^{\prime} 44.59^{\prime} \mathrm{N}$, 2919'20.27' E, 0 m;

- Lacul Roşu (near the Red Lake), 4502'46.98'N, 29॰31'26.36'"E, 0 m;

- $1 \mathrm{~km}$ NW of Murighiol, Lacul Porculeţ (lake) $45^{\circ} 3^{\prime} 7.096$ 'N, 29॰9'0.266”'E, 2 m;

- Canalul Magearu (reed areas with shrubs and trees), 4514'25.31'N, 29³0'18.11'E, 2 m;

- Insula Popina, Rezervaţia faunistică Insula Popina (meadows with shrubs), $44^{\circ} 58^{\prime} 02.65^{\prime} \mathrm{N}$, $28^{\circ} 58^{\prime} 50.22^{\prime \prime} \mathrm{E}, 8 \mathrm{~m}$;

- Smârdan, Braţul Măcin (muddy bank of the

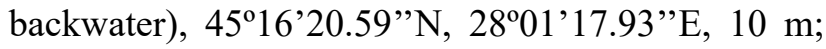
$45^{\circ} 17^{\prime} 51^{\prime \prime} \mathrm{N}, 28^{\circ} 00^{\prime} 21^{\prime \prime} \mathrm{E}$;

- Jijila, râul Jijila (flood catchment area),

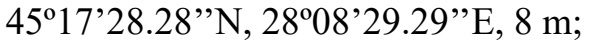

- M-ţii Măcin, to the Peak Ţuţuiatu (meadows with

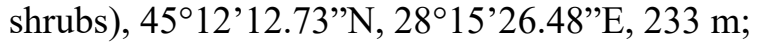

- Parcheş, $45^{\circ} 12^{\prime} 51^{\prime \prime} \mathrm{N}, 28^{\circ} 35^{\prime} 31^{\prime \prime} \mathrm{E}$.

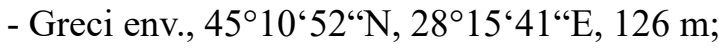

- Măcin, Rezervaţia de la Greci (mixted forest with oak and lime), $45^{\circ} 09^{\prime} 48.25^{\prime \prime} \mathrm{N}, 28^{\circ} 17^{\prime} 37.62^{\prime \prime} \mathrm{E}, 159 \mathrm{~m}$;

- Greci (village), $45^{\circ} 11^{\circ} 40.52^{\prime \prime N}, 28^{\circ} 14^{\prime} 23.22^{\prime \prime} \mathrm{E}, 42 \mathrm{~m}$; M-ţii Măcin, $4.5 \mathrm{~km}$ SE of Greci, $45^{\circ} 09^{\prime} 55^{\prime}$ 'N, $28^{\circ} 16^{\prime} 56^{\prime \prime} \mathrm{E}, 109 \mathrm{~m}$;

- $6 \mathrm{~km} \mathrm{~W}$ of Somova, 45 $10^{\prime} 41^{\prime \prime} \mathrm{N}, 28^{\circ} 34^{\prime} 57^{\prime \prime} \mathrm{E}, 117 \mathrm{~m}$;

- Sălcioara, Lacul Sălcioara (lakeside tall-herb

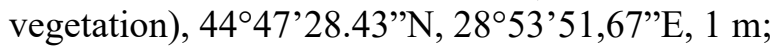

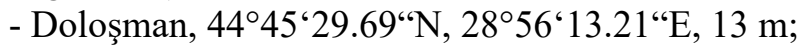

- Iancina (meadws with shrubs and trees),

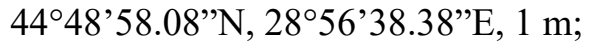

- Dealul Taşburun (meadows), 4451'59.31”N, 2853'08.54'E, $17 \mathrm{~m}$;

- Nifon, pârâul Pârlita valley, 4509'28.80”N, $28^{\circ} 24^{\prime} 08.40^{\prime \prime}$ 'E, $125 \mathrm{~m}$;

- $\mathrm{N}$ from Nifon (222A road, roadside weedy), $45^{\circ} 10^{\prime} 09.45^{\prime \prime} \mathrm{N}, 28^{\circ} 22^{\prime} 07.52^{\prime \prime} \mathrm{E}, 125 \mathrm{~m}$;

- M-ţii Măcin, Nifon (forest), 45¹2'17.30”N, 2820'14.90'"E, $156 \mathrm{~m}$;

- M-ţii Măcin, 2 km NW Nifon, pârâul Curături, $45^{\circ} 10^{\prime} 32^{\prime \prime} \mathrm{N}, 28^{\circ} 21^{\prime} 39^{\prime \prime} \mathrm{E}$;

- M-ţii Măcin, Hamcearca, Culmea Cardonului (the Peak Cardonului, oak forests with glades), $44^{\circ} 05^{\prime} 56.62^{\prime}$ 'N, 28²2'57.97’'E, $238 \mathrm{~m}$;

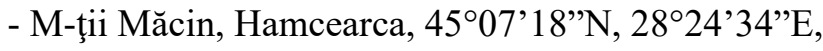
$191 \mathrm{~m}$;

- S from Hamcearca (222A road, wooded pasture), $45^{\circ} 06^{\prime} 10.29^{\prime}$ 'N, 28 24'27.53'"E, $107 \mathrm{~m}$;

- Turcoaia, $6 \mathrm{~km}$ from Cerna to NW (rockgrass),

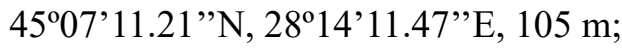

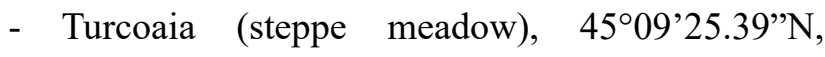
2811'57.03'"E, $35 \mathrm{~m}$;

- Horia, Praporgescu (oak forest and meadows), $45^{\circ} 00^{\prime} 59.17^{\prime \prime} \mathrm{N}, 28^{\circ} 23^{\prime} 40.38^{\prime \prime} \mathrm{E}, 176 \mathrm{~m}$;

- S of Horia, 44 ${ }^{\circ} 59^{\prime} 45.3^{\prime \prime} \mathrm{N}, 28^{\circ} 27^{\prime} 03.7^{\prime \prime} \mathrm{E}, 201 \mathrm{~m}$;

- $2,7 \mathrm{~km}$ from Horia to $\mathrm{S}$ (sikerts of the forest), $44^{\circ} 59^{\prime} 55.56^{\prime \prime} \mathrm{N}, 28^{\circ} 26^{\prime} 56,99^{\prime \prime} \mathrm{E}, 230 \mathrm{~m}$;

- 1,5 km N of Horia, Lacul Horia (lake), $45^{\circ} 03^{\prime} 11^{\prime \prime} \mathrm{N}$, $28^{\circ} 25^{\prime} 27^{\prime \prime} \mathrm{E}, 62 \mathrm{~m}$; 4503'22.2”N, 28`25'23.2”'E, $67 \mathrm{~m}$; - W of Horia, $45^{\circ} 01^{\prime} 05.3^{\prime \prime} \mathrm{N}, 28^{\circ} 25^{\prime} 19.6^{\prime \prime} \mathrm{E}, 89 \mathrm{~m}$;

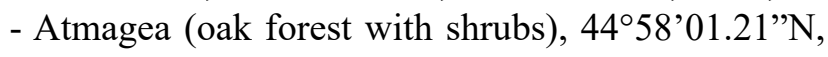
2825'31.52”'E, $227 \mathrm{~m}$;

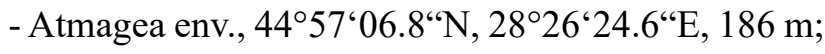
- Ciucurova env., $44^{\circ} 54^{\circ} 07^{\prime \prime} \mathrm{N}, 28^{\circ} 30^{\prime} 14^{\prime \prime} \mathrm{E}, 124 \mathrm{~m}$;

- NE of Topolog, 445' $12.1^{\prime \prime} \mathrm{N}, 28^{\circ} 25^{\prime} 18.7^{\prime \prime} \mathrm{E}, 127 \mathrm{~m}$; - Slava Rusă (mixed deciduous forest), $44^{\circ} 51^{\prime} \mathrm{N}$,

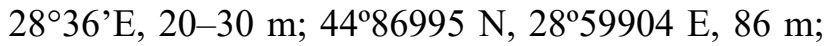
$44^{\circ} 51^{\prime} 47.2^{\prime \prime} \mathrm{N}, 28^{\circ} 38^{\prime} 13.2^{\prime \prime} \mathrm{E}, 114 \mathrm{~m}$; 4450'26.11'N, $28^{\circ} 36^{\prime} 01.78^{\prime \prime} \mathrm{E}, 117 \mathrm{~m}$;

- $2 \mathrm{~km}$ from Slava Rusă to NE (wooded pasture), $44^{\circ} 52^{\prime} 10.05^{\prime \prime} \mathrm{N}, 28^{\circ} 38^{\prime} 51.37^{\prime \prime} \mathrm{E}, 152 \mathrm{~m}$;

- Babadag (forest), 44⒌ $51^{\prime} 58.54^{\prime \prime} \mathrm{N}, 28^{\circ} 41^{\prime} 17.87^{\prime \prime} \mathrm{E}$, $70 \mathrm{~m} ; 44^{\circ} 53^{\circ} \mathrm{N}, 28^{\circ} 53^{\circ} \mathrm{E}, 100 \mathrm{~m} ; 44^{\circ} 49^{\circ} 09.03^{\prime \prime} \mathrm{N}$, $28^{\circ} 41^{\prime} 13.47^{\prime \prime} \mathrm{E}, 140 \mathrm{~m}$;

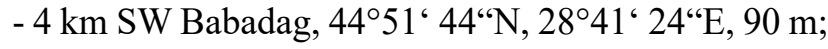
- Codru Monastery (dry open woodland), $44^{\circ} 53^{\circ} \mathrm{N}$, $28^{\circ} 42^{\circ} \mathrm{E}, 25-30 \mathrm{~m} ; 44^{\circ} 81474 \mathrm{~N}, 28^{\circ} 68818 \mathrm{E}, 120 \mathrm{~m}$; $44^{\circ} 48^{\prime} 59.31^{\prime \prime N} \mathrm{~N}, 28^{\circ} 41^{\prime} 21.84^{\prime \prime} \mathrm{E}, 120 \mathrm{~m}$;

- Codru env. (seecoast), $44^{\circ} 49^{\circ} 11^{\prime \prime} \mathrm{N}, 28^{\circ} 41^{\prime} 28^{\prime \prime} \mathrm{E}$, $140 \mathrm{~m}$;

- Enisala, $44^{\circ} 53^{\circ} \mathrm{N}, 28^{\circ} 50^{\circ} \mathrm{E}, 10-20 \mathrm{~m}$;

- Enisala (wooded pasture), $44^{\circ} 51^{\prime} 55.53{ }^{\prime \prime} \mathrm{N}$, $28^{\circ} 49^{\prime} 53,17^{\prime \prime} \mathrm{E}, \quad 60 \mathrm{~m}$; $\quad 44^{\circ} 51^{\prime} 39.50^{\prime \prime} \mathrm{N}$, 2849'57.70'”, 80 m;

- Enisala (castle), $44^{\circ} 53^{\circ} 04.32^{\prime \prime N} \mathrm{~N}, 28^{\circ} 50^{\circ} 11.25^{\prime \prime} \mathrm{E}$, $70 \mathrm{~m} ; 4^{\circ} 53007 \mathrm{~N}, 28^{\circ} 50061 \mathrm{E}, 80 \mathrm{~m}$;

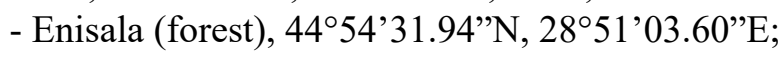

- Enisala, Cetatea Heraclea (arid grass), 44 ${ }^{\circ} 52^{\prime} 59.50^{\prime \prime} \mathrm{N}, 28^{\circ} 50^{\prime} 12.20^{\prime \prime} \mathrm{E}, 65 \mathrm{~m}$; 
- Caugagia, $44^{\circ} 87^{\prime} \mathrm{N}, 28^{\circ} 65^{\prime} \mathrm{E}, 168 \mathrm{~m}$;

- $2.5 \mathrm{~km}$ from Stejaru to NW (wooded pasture),

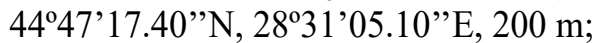

- Stejaru env., $44^{\circ} 47^{\circ} 06^{\prime \prime} \mathrm{N}, 28^{\circ} 32^{\prime} 23^{\prime \prime} \mathrm{E}, 230 \mathrm{~m}$;

- 1,5 km from Beidaud to SW (pasture), $44^{\circ} 42^{\prime} 07.10^{\prime \prime} \mathrm{N}$, 283'02.70'”, $145 \mathrm{~m}$.

Constanţa County:

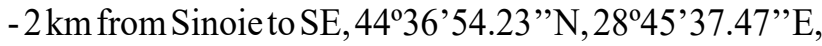
$0 \mathrm{~m}$;

- Sinoie, Grindul Lupilor (wet meadow with tall vegetation) $44^{\circ} 37^{\prime} 10.23^{\prime \prime} \mathrm{N}, 28^{\circ} 48^{\prime} 43.88^{\prime \prime} \mathrm{E}, 0 \mathrm{~m}$;

- Istria, Cetatea Histria (wet meadow with tall-herb vegetation), $44^{\circ} 32^{\circ} 44.07^{\prime \prime} \mathrm{N}, \quad 28^{\circ} 45^{\circ} 39.42^{\prime \prime} \mathrm{E}, 1 \mathrm{~m}$; $44^{\circ} 32^{\circ} 48.60^{\prime \prime} \mathrm{N}, \quad 28^{\circ} 45^{\circ} 38,40^{\prime \prime} \mathrm{E}, 1 \mathrm{~m} ; \quad 44^{\circ} 32.2^{\circ} \mathrm{N}$, $28^{\circ} 44.2^{\circ} \mathrm{E}, 5 \mathrm{~m}$; $44^{\circ} 32^{\circ} 42.14^{\prime \prime} \mathrm{N}, 28^{\circ} 46^{\circ} 02.65^{\circ} \mathrm{E}, 0 \mathrm{~m}$; $44^{\circ} 32^{\circ} 40^{\circ} \mathrm{N}, 28^{\circ} 46^{\circ} 15^{\prime \prime} \mathrm{E}, 0 \mathrm{~m}$; $44^{\circ} 54468 \mathrm{~N}, 28^{\circ} 76063 \mathrm{E}$, $4 \mathrm{~m}$;

- Istria env., Grindul Saiele, Cetatea Histria, 4432'36.33”'N, 2846'19.83”'E, $1 \mathrm{~m}$;

- $9 \mathrm{~km}$ E of Istria, Cetatea Histria, $44^{\circ} 32^{\prime} 42.14^{\prime} \mathrm{N}$, $28^{\circ} 46^{\prime} 02.65^{\prime \prime} \mathrm{E}, 0 \mathrm{~m}$;

- Vadu, Grindul Chituc (coast), 4426’35.2”N, $28^{\circ} 46^{\prime} 50.2^{\prime \prime}$ 'E, $1 \mathrm{~m}$;

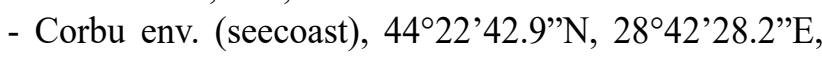
$11 \mathrm{~m}$;

- Tariverde env., $44^{\circ} 33^{\circ} 40.2^{\prime \prime} \mathrm{N}, 28^{\circ} 34^{\prime} 58.8^{\prime \prime} \mathrm{E}, 62 \mathrm{~m}$;

- $3 \mathrm{~km} \mathrm{~W}$ of Crucea, Allah Bair (Nature Reserve),

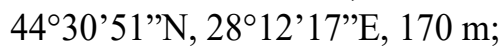

- 5 km SW Gura Dobrogei, above Peştera "La Adam", SW end of gorge, $44^{\circ} 27^{\prime} 52^{\prime \prime} \mathrm{N}, 28^{\circ} 28^{\prime} 17^{\prime \prime} \mathrm{E}, 40 \mathrm{~m}$; $44^{\circ} 27^{\prime} 51^{\prime \prime} \mathrm{N}, 28^{\circ} 28^{\prime} 17^{\prime \prime} \mathrm{E}, 50 \mathrm{~m}$;

- 4,5 km SW Gura Dobrogei, 0,6 km WSW Peştera Liliecilor, p. Târguşorul, bushy wall, $44^{\circ} 27^{\prime} 56^{\prime \prime} \mathrm{N}$, $28^{\circ} 28^{\prime} 28^{\prime \prime}$ 'E, $30 \mathrm{~m}$;

- Gura Dobrogei (rockgrass), $44^{\circ} 27^{\circ} 54.58^{\prime \prime} \mathrm{N}$, $28^{\circ} 29^{\prime} 15.12^{\prime \prime} \mathrm{E}, 65 \mathrm{~m}$; $44^{\circ} 28^{\circ} \mathrm{N}, 28^{\circ} 31^{\circ} \mathrm{E}, 100 \mathrm{~m}$;

- Cheia, Cheile Dobrogei (Dobrogea gorge), $44^{\circ} 95^{\circ} \mathrm{N}$, $28^{\circ} 44^{\circ} \mathrm{E}, 34 \mathrm{~m}$; $44^{\circ} 30^{\prime} 19.09^{\prime \prime} \mathrm{N}, 28^{\circ} 25^{\circ} 49.91^{\prime \prime} \mathrm{E}, 57 \mathrm{~m}$;

- Cheia env., Cheia Nature Reserve., $44^{\circ} 29^{\circ} 25.3^{\prime \prime} \mathrm{N}$, $28^{\circ} 26^{\circ} 14.6^{\prime \prime} \mathrm{E}, 132 \mathrm{~m}$;

- Cheia, Nature Reserve, $2 \mathrm{~km} \mathrm{~S}$ of Cheia (steppe), $44^{\circ} 29^{\prime} \mathrm{N}, 28^{\circ} 25^{\prime} \mathrm{E}$;

- Grădina env., 44³3 ‘33.5“N, 28²6‘39.1“E, 71 m;

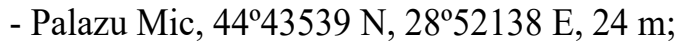

- Murfatlar, Fântâniţa (dry slope steppe with sparse woods), $44^{\circ} 16026 \mathrm{~N}, 28^{\circ} 52138 \mathrm{E}, 25 \mathrm{~m} ; 4^{\circ} 09^{\prime} 38.28^{\prime \prime} \mathrm{N}$, $28^{\circ} 23$ '26,32” E, $41 \mathrm{~m}$;

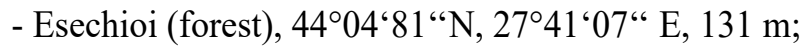

- Esechioi (semi-dry slope steppic grassland with shrub

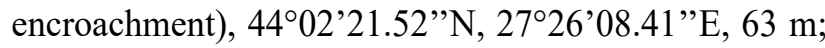

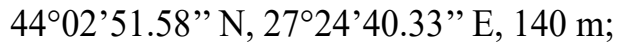

- Esechioi, Lacul Bugeac, towards the south Lake Bugeac (semi-dry slope steppic grassland with shrub),

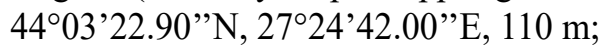

- Băneasa, Canaraua Fetii (quarry), $44^{\circ} 16^{\prime} 01^{\prime \prime N}$, 2838'80"'E, 44 m;

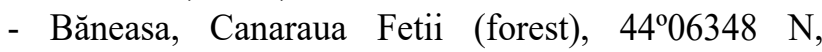
$27^{\circ} 65113$ E, $59 \mathrm{~m} ; 4^{\circ} 04^{\prime} 49.75^{\prime \prime} \mathrm{N}, 27^{\circ} 38^{\prime} 36.74^{\prime \prime} \mathrm{E}, 30$ m; 440' 14.51'”N, 27³8’32.39”'E, 30 m;

- Băneasa, Canaraua Fetii (forest edge), 4403'50.03”N, $27^{\circ} 38^{\prime} 41.77^{\prime \prime} \mathrm{E}, 39 \mathrm{~m}$;

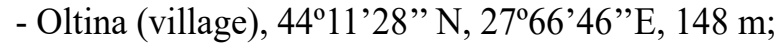

- Ostrov, Lacul Bugeac (lakeside tall-herb vegetation),

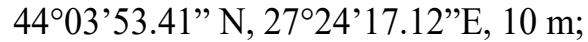

- Ostrov, Lacul Bugeac, northward Lake Bugeac (roadside tall-herb vegetation), $44^{\circ} 04^{\prime} 42.59^{\prime \prime} \mathrm{N}$, 2723'35.54'”, 45 m;

- Bugeac (under the evening street lights), $44^{\circ} 05^{\prime} 57.24^{\prime \prime} \mathrm{N}$, $27^{\circ} 25^{\prime} 55,73$ ”'E, $20 \mathrm{~m}$;

- Negureni, Pădurea Valea Cişmelelor (moufflon forest), $44^{\circ} 08802 \mathrm{~N}, 27^{\circ} 74871 \mathrm{E}, 95 \mathrm{~m}$;

- Ion Corvin (dry slope steppe with sparse woods), $44^{\circ} 16^{\prime} 27,50^{\prime \prime} \mathrm{N}, 27^{\circ} 51^{\prime} 02,60^{\prime \prime} \mathrm{E}, 120 \mathrm{~m}$;

- 3,5 km SW of Rasova, Lacul Baciului (lake), $44^{\circ} 13^{\prime} 03^{\prime}$ 'N, 275' $40^{\prime \prime} \mathrm{E}, 40 \mathrm{~m}$;

- Cochirleni, $44^{\circ} 28^{\circ} 48^{\prime \prime N} \mathrm{~N}, \quad 28^{\circ} 01^{\prime} 17^{\prime \prime} \mathrm{E}, 20 \mathrm{~m}$; $44^{\circ} 28^{\circ} 49^{\prime \prime} \mathrm{N}, 28^{\circ} 01^{\prime} 19^{\prime \prime} \mathrm{E}, 41 \mathrm{~m}$;

-Mangalia env., Camping Popas Zodiac, 4351‘32.933“N, $28^{\circ} 35^{\circ} 56.257^{\prime \prime} \mathrm{E}, 2 \mathrm{~m}$;

- Mangalia env., Camping Saturn, 4349'49.454”N, 2834'50.925'"E, 2 m;

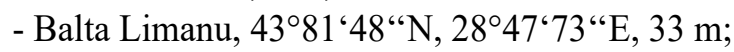

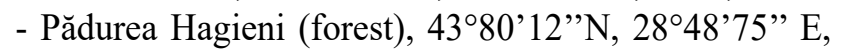
$24 \mathrm{~m}$.

\section{Abbreviations of the collectors' names:}

Ada Maria Andrei (AA), A. Bulumac (AB), Alin Constantinescu (AC), Anneliese KonnerthIONESCU (AKI), A. CARASTATE (ACa), Arnold-Lucien Montandon (ALM), Alexandru MarinesCu (AMa), Aurora Marcu (AM), Angela Petrescu (AP), Dr. Aurelian Popescu-GorJ (APG), Adrian RuiCĂNESCU (AR), A. STÃNeSCU (AS), Atena RoşCa (AtR), Adriana VÃraru (AV), Cristina BAN (CB), Roberto CALDARA (RC), Cristina Calefariu (CC), Roman Borovec (RB), Cristina Hoinic (CH), Carmen IrInCU (CI), Corneliu PÂrvu (CP), C. Roman (CR), Cecilia ŞERbAN (CŞ), Csősz Sándor (CsS), Dorel RuşTi (DR), Eduard Fleck (EF), Elena Iorgu (EI), Elena PISICĂ (EP), Fetykó Kinga (FK), Gabriela Andrei (GA), Gheorghe Boguleanu (GB), Gabriela CHIŞAMERA (GC), Dr. Gozmány László (GL), Gabriela Patriche (GP), Igor CeiANu (IC), Igor SienkiEwICZ (IS), Ioana Matache (IM), Ion DrăGhia (ID), Ionela Stanciu (IoS), Iorgu Petrescu (IP), Ionuţ Şt. Iorgu (IŞI), IzsÁK Zoltán (IZ), Jiří KRÁTKÝ (JK), Kocs Irén (KI), Kocs János (KJ), Michael KošŤál (MK), KresL Petr (KP), Liviu Moscaliuc (LM), ? (LS), Mircea ANDreI (MA), Margareta Cantoreanu (MaC), M. BarCon (MB), Mihaela Creţu (MC), Mariana Foaltin (MF), Makranczy György (MGy), Mihai Stănescu (MiS), Mihaela Minai (MM), Melanya Stan (MS), Matei Tălpeanu (MT), Medeea Weinberg (MW), Năstase RĂduleț (NR), NÉdli Judit (NJ), Nicolae GÂldEAN (NG), Dr. Nicolae Săvulescu (NS), Orosz András (OA), Podlussány Attila (PA), Pavel Filip (PF), 
P. Gherghel (PG), Pelikán Jan (PJ), RecueroGIL, E. (RE), Rozner István (RI), R. ŠKodA (RŠ), Sekerka LuKáš (SL), Rodica SERAFIM (RS), ŠKoda Richard (SR), Somai László (SoL), Şerban Procheş (ŞP), Ştefan Negru (ŞN), SzÉKely Kálmán (SzK), SzÉKely Levente (SzL), TallósI Béla (TB), Vladimir BrĂdescu (VB), Viorel GAVRILĂ (VG), Vörös Judit (VJ), Victoria Raica (VR), Xenia Palade (XP), Xenia Scobiola (XS), Cristian Sitar (CS), David Dradomir Cosmin (DDC), TeOdor Lucian Alexandru (TLA).

\section{Abbreviations of the museum collections:}

(Coll. Mus. R. AngheluŢă) - Complexul Muzeal de Ştiinţele Naturii „Răsvan Angheluţă” Galaţi („Răsvan ANGHeluță” Museum Complex of Natural Sciences Galaţi).

(Coll. Mus. G. AnTIPA) - Muzeul Naţional de Istorie Naturală „Grigore Antipa” Bucureşti ("Grigore

Antipa" National Museum of Natural History

Bucharest).

(Coll. Hung. Nat. Hist. Mus.) - Magyar

Természettudományi Múzeum Budapest (Hungarian Museum of Natural History Budapest).

\section{Results}

Combining the data from the literature, the collections and the collecting activities, presently 724 species in 5 families and in 234 genera are known from the Danube-Delta and Dobruja.

While we didn't succeed collecting all the 115 species listed in the literature, we managed to collect 270 species new to the Danube-Delta and Dobruja, 50 species new to the fauna of Romania (marked by an asterix $(*)$ in the species list) and 2 species new to science (the description of the species is in progress). A closer scrutiny will be needed in the case of 4 variants; the names of these appear unbolded on the species list.

The names of the species and their taxonomical classificaton following the Cooperative Catalogue of Palaearctic Coleoptera Curculionoidea (AlonsoZARAZAGA et al. 2017). We were also guided by the checklist of Romanian Curculionoidea (TEODOR and ANTONIE Vlad 2007).

COLEOPTERA

CURCULIONOIDEA

NEMONYCHIDAE BEDEL, 1882

NEMONYCHINAE BEDEL, 1882

Nemonyx lepturoides (FABRICIUS, 1801)

New data: Tulcea County: Enisala (wooded pasture), 24-25.V.2013, leg. PA, 4.VI.2018, leg. FK, KI, KJ PA; Constanţa County: Murfatlar, Fântâniţa, 4-5. VII.2014, leg. KI, OA, PA, SzL.

ANTHRIBIDAE BILlBERG, 1820

ANTHRIBINAE BILLBERG, 1820
Piesocorynini Valentine, 1960

Phaenotherium pulszkyi J. FRIVALDSZKY, 1877

New data: Tulcea County: Slava Rusă, 4.V.2009, leg. JK, 24-25.V.2013 leg. KI, PA, RI, SzL, 1-3.V.2014, leg. KI, IZ, PA, SzL; Codru env., 3.V.2009, leg. JK; NE of Topolog, 4.V.2009, leg. JK; Greci env.,6.V.2009, leg. JK; Babadag (forest), 30.IV.2014, leg. KI, IZ, PA, SzL; 2,5 km from Stejaru to NW, 25.VI.2017, leg. KI, KJ, PA, TB; 2,7 km from Horia to S, 28.VI.2017, leg. KI, KJ, PA, TB.

Platyrhinini IMHOFF, 1856

Platyrhinus resinosus (SCOPOLI, 1763)

New data: Tulcea County: M-ţii Măcin, Mănăstirea Cocoş, 9.VI.1973, leg. AC (Coll. Mus. G. AnTIPA) - Constanţa County: Băneasa, Canaraua Fetii, 23.V.2015, leg. PA.

Platystomini PIERCE, 1916

Platystomos albinus (LiNNÉ, 1758)

New data: Tulcea County: Babadag (forest), 22.IX.2015, leg. KI.

Zygaenodini LACORDAIRE, 1865

Dissoleucas niveirostris (FABRICIUS, 1798)

New data: Tulcea County: Slava Rusă, 4.V.2009, leg. JK, 1-3.V.2014, leg. KI, IZ, PA, SzL; Babadag (forest), 22.IX.2015, leg. KI, KJ, PA; 2,5 km from Stejaru to NW, 25.VI.2017, leg. TB; Constanța County: Esechioi (forest), 1-2.IV.2016, leg. FK, KI, PA, SzL; Băneasa, Canaraua Fetii (forest), 17.IX.2016, leg. KI, KJ, PA, TB.

*Noxius curtirostris (MULSANT \& REY, 1861)

New data: Tulcea County: Jijila, 19.VI.2019, leg. TB.

Rhaphitropis marchicus (HERBST, 1797)

New data: Constanţa County: Furnica, Rezervaţia Dumbrăveni, 13-14.V.1995, leg. CP (Coll. Mus. G. ANTIPA).

URODONTINAE C. G. ThOMSON, 1859

*Bruchela cana (KÜSTER, 1848) (fig. 2)

New data: Tulcea County: Enisala, 24-25.V.2013, leg. KI, PA, RI, SzL.

*Bruchela concolor (FÅHRAEUS, 1839)

New data: Tulcea County: Celic Dere, 20-24.V.1994, leg. CP, 20.V.1997, leg. RS, 20.V.1997, leg. CH (Coll. Mus. G. ANTIPA) - Enisala, 24-25.V.2013, leg. KI, PA, RI, SzL.

Bruchela conformis (SUFFRIAN, 1845)

Published data: RADUTA 2014

Bruchela kaszabi (STREJČEK, 1973) (= Urodon orientalis STREJČEK, 1982)

New data: Tulcea County: Babadag (forest), 2.IV.2005, leg. CsS, 30.IV.2014, leg. KI, IZ, PA, SzL; M-ţii Măcin, Valea Fagilor (quarry), 30.V.2005, leg. 


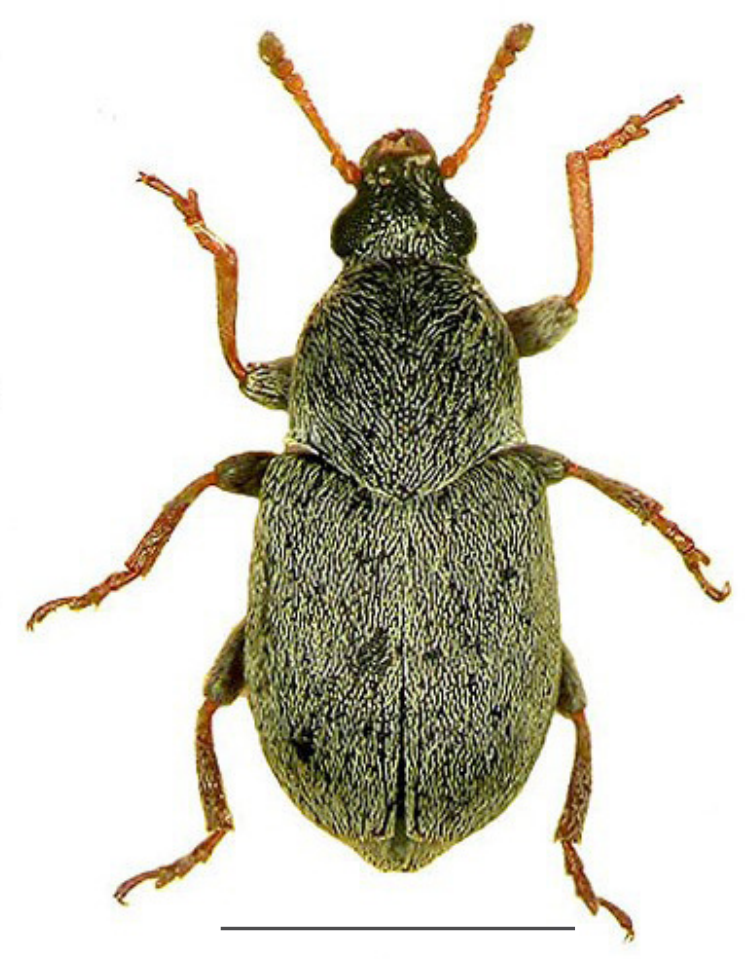

Fig. 2. Bruchela cana (KüSTER, 1848). Scale bar: $1 \mathrm{~mm}$. Photo Zoltán GYÖRGY

CsS; $3 \mathrm{~km}$ W of Crucea, Allah Bair (Nature Reserve), 1.V.2009, leg. JK; Enisala, 24-25.V.2013, leg. KI, PA, RI, SzL, 29.IV-3.V.2014, leg. KI, IZ, PA, SzL; Enisala (castle), 21.V.2015, leg. PA, RI, SzK, SzL, 10.V.2017, leg. FK, KI, KJ, PA; M-ţii Măcin, Greci, 7.V.2017, leg. FK, KI, KJ, PA, 8.V.2017, leg. FK, KI, KJ, PA; M-ţii Măcin, 5 km S Greci, 8.V.2017, leg. FK, KI, KJ, PA; Slava Rusă, Uspenia Monastery, 11.V.2017, leg. FK, KI, KJ, PA; Constanţa County: Gura Dobrogei, 26.V.2013, leg. KI, PA, RI, SzL, 30.IV-2.V.2014, leg. KI, IZ, PA, SzL; Istria, Cetatea Histria, 21.V.2015, leg. PA, RI, SzK, SzL, 11.V.2017, leg. FK, KI, KJ, PA; Palazu Mic, 22.V.2015, leg. PA, RI, SzK, SzL; Băneasa, Canaraua Fetii, 23.V.2015, leg. PA, RI, SzK, SzL; Cochirleni, 24.V.2015, leg. PA, RI, SzK, SzL; Sinoie, Grindul Lupilor, 5.VI.2018, leg. FK, KI, KJ, PA; Vadu, Grindul Chituc, 6.VI.2018, leg. FK, KI, KJ, PA.

\section{*Bruchela parvula (MOTSCHULSKY, 1875) (fig. 3)}

New data: Tulcea County: Enisala, 24-25.V.2013, leg. KI, PA, RI, SzL, 29.IV-3.V.2014, leg. KI, IZ, PA, SzL; Codru Monastery, 30.IV-3.V.2014, leg. KI, IZ, PA, SzL; Slava Rusă, 1-3.V.2014, leg. KI, IZ, PA, SzL; Enisala (castle), 10.V.2017, leg. FK, KI, KJ, PA; M-ţii Măcin, Greci, 8.V.2017, leg. FK, KI, KJ, PA; Constanţa County: Agigea, 27.V.1963, leg. XS (Coll. Mus. G. Antipa) - Cheile Dobrogei, 2.V.2014, leg. KI, IZ, PA, SzL; Palazu Mic, 22.V.2015, leg. PA, RI, SzK, SzL; Murfatlar, Fântânița 22.V.2015, leg. PA, RI, SzK, SzL; Băneasa, Canaraua Fetii, 23.V.2015, leg. PA, RI, SzK, SzL; Gura Dobrogei, 12.V.2017, leg. FK, KI, KJ, PA.

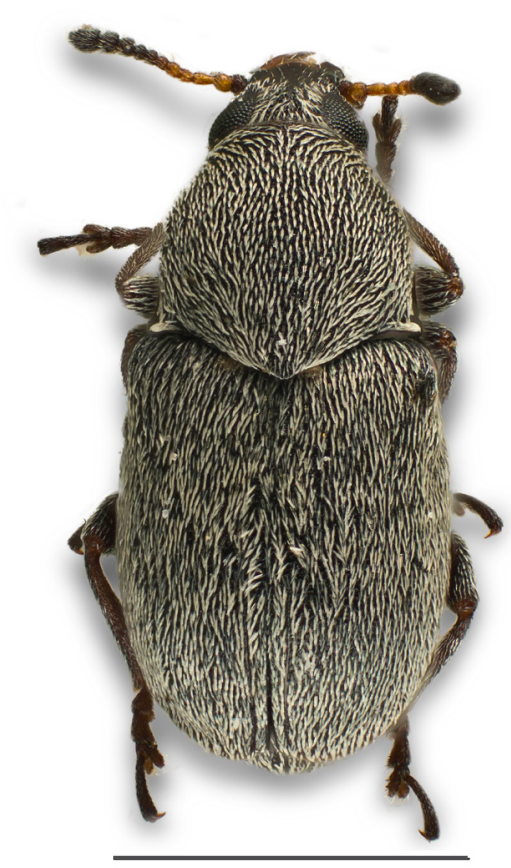

Fig. 3. Bruchela parvula (MotsChulsky, 1875). Scale bar: $1 \mathrm{~mm}$. Photo Zoltán CsATA

Bruchela rufipes rufipes (OLIVIER, 1790)

Published data: RADUTA 2014

New data: Tulcea County: Codru Monastery, 2325.V.2013, leg. KI, PA, RI, SzL, 24-25.VI.2017, leg. KI, KJ, PA, TB, 20.VI.2019, leg. KI, KJ, PA, TB; Enisala, 29.IV-3.V.2014, leg. KI, IZ, PA, SzL; Turcoaia, $6 \mathrm{~km}$ from Cerna to NW, 24.VI.2017, leg. KI, KJ, PA, TB; $1,5 \mathrm{~km}$ from Beidaud to SW, 25.VI.2017, leg. KI, KJ, PA, TB; Constanţa County: Agigea, 18.VII.1964, leg. MW (Coll. Mus. G. AnTIPA) - Gura Dobrogei, 30.IV-2.V.2014, leg. KI, IZ, PA, SzL, 7.VI.2018, leg. FK, KI, KJ PA.

\section{Bruchela schusteri (SCHILSKY, 1912)}

New data: Tulcea County: Codru Monastery, 2325.V.2013, leg. KI, PA, RI, SzL; Turcoaia, 6 km from Cerna to NW, 3.VI.2018, leg. FK, KI, KJ, PA; Constanţa County: Sinoie, Grindul Lupilor, 5.VI.2018, leg. FK, KI, KJ, PA.

\section{Bruchela suturalis (FABRICIUS, 1792)}

Published data: RADUTA 2014

New data: Tulcea County: Greci, 3.VI-7.VII.1964, leg. IS; Cerna, 26.V.2005, leg. IM; Măcin, Valea Suluc, 27.V.2005, leg. IM (Coll. Mus. G. ANTIPA) - M-ţii Măcin, Valea Fagilor (dried lake bed), 30.V.2005, leg. CsS; Codru Monastery, 23-25.V.2013.V, leg. KI, PA, RI, SzL, 30.IV-3.V.2014, leg. KI, IZ, PA, SzL, 19.V.2015, leg. PA, RI, SzK, SzL, 10-11.V.2017, leg. FK, KI, KJ, PA; Enisala, 24-25.V.2013, leg. KI, PA, RI, SzL, 29.IV-3.V.2014, leg. KI, IZ, PA, SzL; Slava Rusă, 24-25.V.2013, leg. KI, PA, RI, SzL, 1-3.V.2014, leg. KI, IZ, PA, SzL, 20.V.2015, leg. PA, RI, SzK, 
SzL; Babadag (forest), 30.IV.2014, leg. KI, IZ, PA, SzL; M-ţii Măcin, Greci, 7.V.2017, leg. FK, KI, KJ, PA, 8.V.2017, leg. FK, KI, KJ, PA; Enisala (castle), 20.VI.2019, leg. KI, KJ, PA, TB; Constanţa County: Agigea, 27.V.1963, leg. XS; Hagieni, 12.VII.1964, leg. IS; Băneasa, Canaraua Feti, 20.V.1993, leg. CP (Coll. Mus. G. ANTIPA) - Gura Dobrogei, 26.V.2013, leg. KI, PA, RI, SzL, 30.IV-2.V.2014, leg. KI, IZ, PA, SzL; Palazu Mic, 22.V.2015, leg. PA, RI, SzK, SzL; Cochirleni, 24.V.2015, leg. PA, RI, SzK, SzL; Sinoie, Grindul Lupilor, 5.VI.2018, leg. FK, KI, KJ, PA, 22.VI.2019, leg. KI, KJ, PA, TB; Crucea, 9.VI.2018, leg. FK, KI, KJ, PA.

ATTELABIDAE BILlBerg, 1820

ATTELABINAE BILLBERG, 1820

Apoderini JEKEL, 1860

Apoderina JEKEL, 1860

Apoderus coryli (LinNÉ, 1758)

Published data: FLECK 1905

Attelabini BiLlBerg, 1820

Attelabus nitens (SCOPOLI 1763)

Published data: JaQuet 1899b, Fleck 1905, Negru and RoşCa 1967, Raduta 2014

New data: Tulcea County: C. A. Rosetti, 07. 1950, leg. IC; Pădurea Babadag, 20.VI.1963, leg. XS, 20.VI.1965, leg. XS; M-ţii Măcin, Mănăstirea Cocoş, 12-17.V.1975, leg. ID (Coll. Mus. G. ANTIPA) - M-ții Măcin, 5 km S Greci, 8.V.2017, leg. FK, KI, KJ, PA; M-ţii Măcin, to the Peak Ţuţuiatu, 28.IV.2010, leg. AR; Constanţa County: Oltina, 26.VI.1956, leg. NS; Băneasa, Canaraua Fetii, 29.VI.1956, leg. NS, 11.IV.1964, leg. NS, 1.V.1965, leg. XS, 18.V.1993, leg. CP; Băneasa, 28.V.1962, leg. NS, 24.V.1964, leg. XS; Comorova, 2.V.1962, leg. NS (Coll. Mus. G. ANTIPA).

RHYNCHITINAE GISTEL, 1848

Byctiscini Voss, 1923

Byctiscina Voss, 1923

Byctiscus betulae (LINNÉ, 1758)

Published data: FLECK 1905

New data: Tulcea County: Valea Teilor, 22.V.1997, leg. CH; Celic Dere, 27.V.2005, leg. RS (Coll. Mus. G. Antipa); M-ții Măcin, Pădurea Căprioarei $(150$ m), 16.VI.2005, leg. GP (Coll. Mus. R. ANGHELUȚǍ) Măcin, Rezervaţia de la Greci, 23.IV.2009, leg. TLA; Babadag (forest), 13.IX.2016, leg. KI; $2 \mathrm{~km}$ from Slava Rusă to NE, 3.VI.2018, leg. FK, KI, KJ, PA.

Deporaini Voss, 1929

Deporaina Voss, 1929

Deporaus (Deporaus) betulae (LinNÉ, 1758)

Published data: FLECK 1905

Rhynchitini GISTEL, 1848

Involvulus (Teretriorhynchites) icosandriae icosandriae (SCOPOLI, 1763) (= caeruleus DEGEER, 1775)
Published data: FLeCK 1905

New data: Tulcea County: Slava Rusă, 25.V.2013, leg. KI.

\section{Involvulus (Teretriorhynchites) pubescens (FABRICIUS 1775) \\ Published data: JAQUET 1900b}

Lasiorhynchites (Coccygorrhynchites) sericeus (HERBst, 1797)

New data: Tulcea County: Măcin, leg. ALM (Coll. Mus. G. AntiPA); Constanţa County: Băneasa, Canaraua Fetii, 29.VI.1956, leg. NS (Coll. Mus. G. ANTIPA).

Lasiorhynchites (Nelasiorhynchites) comatus (Gyllenhal, 1833) (= olivaceus Gyllenhal, 1833) New data: Tulcea County: Codru Monastery, 30-31. III.2016, leg. FK, KI, PA, SzL.

Mecorhis (Mecorhis) ungarica (HERBST, 1783)

Published data: FLECK 1905, Negru and RoşCa 1967, RADUTA 2014

New data: Tulcea County: Beştepe, 14.VI.1993, leg. TLA; Constanţa County: Mangalia, Pădurea Comorova, 2.VI.1962, leg. APG; Mangalia, Păd. Hagieni, 5.VI.1962, leg. APG, 06. 06. 1965, leg. ŞN; Esechioi, 25.V.1962, leg. NS (Coll. Mus. G. ANTIPA) Gura Dobrogei, 26.V.2013, leg. RI, 30.IV-2.V.2014, leg. KI, IZ, PA, SzL, 25, 27.VI.2017, leg. TB.

Mecorhis (Pseudomechoris) aethiops (BACH, 1854) Published data: JAQUET 1899a, FLECK 1905

New data: Constanţa County: Hârşova, leg. ALM (Coll. Mus. G. ANTIPA) - Murfatlar, Fântâniţa, 7-8. VI.2018, leg. FK, KI, KJ, PA.

Neocoenorrhinus (Neocoenorhinidius) interpunctatus (STEPHENS, 1831)

New data: Tulcea County: Babadag, Visterna, 15.V.1963, leg. IC (Coll. Mus. G. ANTIPA) - M-ții Măcin, Valea Fagilor, 30.V.2005, leg. CsS; Codru Monastery, 30.IV-3.V.2014, leg. KI, IZ, PA, SzL, 30 31.III.2016, leg. FK, KI, PA, SzL; Constanța County: Gura Dobrogei, 30.IV-2.V.2014, leg. KI, IZ, PA, SzL; Esechioi (forest), 1-2.IV.2016, leg. FK, KI, PA. SzL.

Neocoenorrhinus (Neocoenorhinidius) pauxillus (GERMAR, 1823)

Published data: FLECK 1905

New data: Tulcea County: Babadag, Visterna, 16.V.1963, leg. IC (Coll. Mus. G. ANTIPA) - Stejaru env., 5.V.2009, leg. JK; Babadag (forest), 23.V.2013, leg. KI; Slava Rusă, 1-3.V.2014, leg. KI, IZ, PA, SzL; Constanţa County: Gura Dobrogei, 28-29.IV.2012, leg. KI, 30.IV-2.V.2014, leg. KI, IZ, PA, SzL.

Neocoenorrhinus (Neocoenorrhinus) germanicus (HERBST, 1797) 
Published data: JAQUET 1900a

New data: Tulcea County: M-ţii Măcin, Pricopan, 1.VI.2005, leg. CsS; Slava Rusă, 1-3.V.2014, leg. KI, IZ, PA, SzL; M-ții Măcin, Greci, 8.V.2017, leg. FK, KI, KJ, PA; N from Nifon, 23.VI.2017, leg. KI, KJ, PA, TB; Constanţa County: Ostrov, 2.IV.2016, leg. FK, KI, PA. SzL; Esechioi (forest), 1-2.IV.2016, leg. FK, KI, PA. SzL; Pecineaga (forest), 14.V.2017, leg. FK, KI, KJ, PA; Cobadin, 14.V.2017, leg. FK, KI, KJ, PA.

\section{Neocoenorrhinus (Schoenitemnus) minutus (Herbst, 1797) (= Coenorrhinus aeneovirens Marsham, 1802) \\ Published data: Negru 1957, Negru and RoşCa 1967, RADUTA 2014 \\ New data: Constanţa County: Furnica, Rez. Dumbrăveni, 13-14.V.1995, leg. CP (Coll. Mus. G. Antipa) - Esechioi (forest), 1-2.IV.2016, leg. FK, KI, PA. SzL; Băneasa, Canaraua Fetii (quarry), 2-3. IV.2016, leg. FK, KI, PA, SzL.}

Rhynchites (Epirhynchites) auratus (SCOPOLI, 1763) Published data: Montandon 1887, FleCK 1905, Negru 1957, Negru and RoşCa 1967, Teodor 1993, RADUTA 2014

New data: Constanţa County: Murfatlar, 6.V.1951, leg. NS; Băneasa, Canaraua Fetii, 27.V.1962, leg. NS; Hagieni, 29.V.1962, leg. NS, 21.V.1965, leg. NS, 19.V.1966, leg. NS; Mangalia, Pădurea Comorova, 2.VI.1962, leg. APG; Băneasa, 15.V.1963, leg. NS (Coll. Mus. G. Antipa) - Gura Dobrogei, 28-29. IV.2012, leg. KI; Murfatlar, Fântâniţa, 4-5.VII.2014, leg. KI, OA, PA, SzL, 7-8.VI.2018, leg. FK, KI, KJ, PA; Esechioi (forest), 7.VII.2014, leg. KI, OA, PA, SzL; Bugeac, 2.IV.2016, leg. FK, KI, PA, SzL.

\section{Rhynchites (Epirhynchites) giganteus (SCHÖNHERR, 1832)}

Published data: FleCK 1905, Montandon 1908

New data: Constanţa County: Oltina, Păd. Ciufâtu, 24.IV.1956, leg. APG; Băneasa, Canaraua Fetii, 27.V.1962, leg. NS (Coll. Mus. G. AntIPA) - Gura Dobrogei, 30.IV-2.V.2014, leg. KI, IZ, PA, SzL.

Rhynchites (Rhynchites) bacchus (LINNÉ, 1758) Published data: Montandon 1887, Fleck 1905, Negru 1957, Negru and RoşCa 1967, Raduta 2014 New data: Constanţa County: Pădurea Hagieni, 19.VI.1962, leg. AtR; Băneasa, 15.V.1963, leg. NS; Hagieni, 20.V.1963, leg. NS; Băneasa, C. Fetii, 30.V.1965, XS (Coll. Mus. G. AnTIPA) - Gura Dobrogei, 30.IV-2.V.2014, leg. KI, IZ, PA, SzL; Murfatlar, Fântâniţa 24-25.IX.2015, leg. KI, KJ, PA; Băneasa, Canaraua Fetii (quarry), 2-3.IV.2016, leg. FK, KI, PA, SzL; Bugeac, 2.IV.2016, leg. FK, KI, PA, SzL; Ostrov, 2.IV.2016, leg. FK, KI, PA, SzL; Esechioi (forest), 1-2.IV.2016, leg. FK, KI, PA. SzL.
Tatianaerhynchites aequatus (LINNÉ, 1767)

Published data: Fleck 1905, Negru 1957, Negru and RoşCa 1967, RADUTA 2014

New data: Tulcea County: Babadag, Visterna, 15.V.1963, leg. IC; Cerna, 26.V.2005, leg. CB (Coll. Mus. G. AntIPA) - Măcin, Rezervaţia de la Greci, 23.IV.2009, leg. TLA; M-ţii Măcin, to the Peak Ţuţuiatu, 28.IV.2010, leg. TLA; Atmagea, 23.IV.2009, leg. TLA; Horia, Praporgescu, 23.IV.2009, leg. TLA; Babadag (forest), 23.V.2013, leg. KI; Codru Monastery, 30.IV-3.V.2014, leg. KI, IZ, PA, SzL; Slava Rusă, 1-3.V.2014, leg. KI, IZ, PA, SzL; Constanţa County: Mangalia, Păd. Comorova, 2.VI.1962, leg. APG, 17.VI.1963, leg. NS; Cerna, 26.V.2005, leg. CB (Coll. Mus. G. ANTIPA) - Gura Dobrogei, 28-29.IV.2012, leg. KI, 30.IV-2.V.2014, leg. KI, IZ, PA, SzL; Murfatlar, Fântâniţa, 22.V.2015, leg. PA, RI, SzK, SzL, 7-8.VI.2018, leg. FK, KI, KJ, PA; Esechioi (forest), 1-2.IV.2016, leg. FK, KI, PA, SzL; Băneasa, Canaraua Fetii (quarry), 2-3.IV.2016, leg. FK, KI, PA, SzL; Bugeac, 2.IV.2016, leg. FK, KI, PA, SzL; Cochirleni, 3.IV.2016, leg. FK, KI, PA, SzL; Ostrov, 2.IV.2016, leg. FK, KI, PA, SzL; NeptunOlimp, Pădurea Comorova, 13.V.2017, leg. FK, KI, KJ, PA.

BRENTIDAE BILBERG, 1820

APIONINAE SCHÖNHERR, 1823

Apionini SCHÖNHERR, 1823

Apionina SCHÖNHERR, 1823

Apion frumentarium (LINNÉ, 1758) (= miniatum GERMAR, 1833)

Published data: Montandon 1887, Fleck 1905, Wagner 1910, Negru and Roşca 1967, TeOdor 1993, TeOdor and Manole 1996, Raduta 2014

New data: Tulcea County: Izvoarele, Valea râului Taiţa, 24.V.1997, leg. CP (Coll. Mus. G. ANTIPA) Gorgova, 16.VI.1993, leg. TLA; Canalul Magearu, 17.VI.1993, leg. TLA; Babadag (forest), 22.IX.2015, leg. KI, KJ, PA; Smârdan, Braţul Măcin, 7.V.2017, leg. FK, KI, KJ, PA, 22.VI.2017, leg. KI, KJ, PA, TB; Slava Rusă, Uspenia Monastery, 11.V.2017, leg. FK, KI, KJ, PA; Enisala, 20.VI.2019, leg. KI; Constanţa County: Agigea, 9.VIII.1962, leg. ID; Limanu, Păd. Hagieni, 22.V.1993, leg. CP (Coll. Mus. G. AntIPA) - Băneasa, Canaraua Fetii (quarry), 2-3.IV.2016, leg. FK, KI, PA. SzL; Băneasa, Canaraua Fetii (forest), 17.IX.2016, leg. KI, KJ, PA, TB; Cochirleni, 3.IV.2016, leg. FK, KI, PA. SzL.

Apion haematodes haematodes KIRBY, 1808

(= frumentarium PAYKULL, 1792)

Published data: Negru and RoşCa 1967, Raduta 2014

Aplemonina Kissinger, 1968

*Perapion (Perapion) connexum (SCHILSKY, 1902) (fig. 4)

New data: Tulcea County: 2 km from Slava Rusă to NE, 3.VI.2018, leg. KI. 


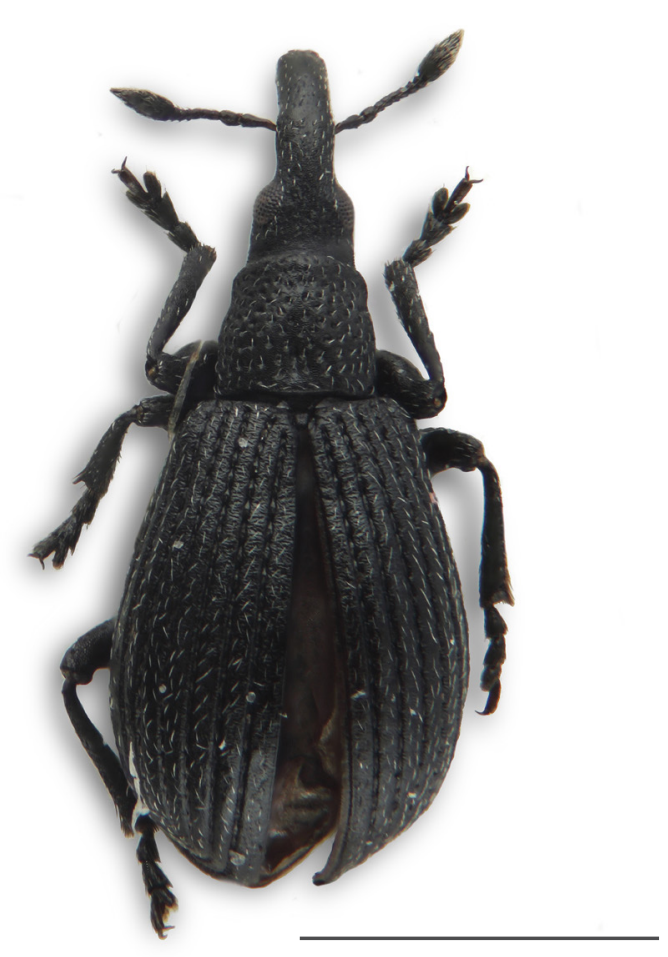

Fig. 4. Perapion (Perapion) connexum (SCHILSKY, 1902). Scale bar: $1 \mathrm{~mm}$. Photo Zoltán CsATA

Perapion (Perapion) curtirostre (GERMAR, 1817) Published data: Montandon 1887, Negru and RoșCa 1967, RADUTA 2014

Perapion (Perapion) marchicum (HERBST, 1797) Published data: Montandon 1887, Fleck 1905, Negru and RoşCA 1967

Perapion (Perapion) violaceum violaceum (KIRBY, 1808)

Published data: WAGNER 1910

New data: Constanţa County: Băneasa, Canaraua Fetii (quarry), 2-3.IV.2016, leg. FK, KI, PA, SzL; Cochirleni, 3.IV.2016, leg. FK, KI, PA, SzL.

Pseudaplemonus artemisiae (MoRAVITZ, 1861)

Published data: KIzUB and SLUTSKy 2018

New data: Constanţa County: Istria env., Cetatea Histria, 18.VII.2009, leg. MK, 20.IX.2010, leg. JK, KP.

Pseudaplemonus externepunctatus (DESBROCHERS des Loges, 1875)

Published data: MontANDON 1908, WAGNER 1910

Pseudoperapion brevirostre (HERBST, 1797)

New data: Tulcea County: Codru Monastery, 2325.V.2013, leg. KI, PA, RI, SzL; Turcoaia, 6 km from Cerna to NW, 24.VI.2017, leg. KI, KJ, PA, TB, 3.VI.2018, leg. KI; Constanța County: Gura Dobrogei, 25-27.VI.2017, leg. KI, KJ, PA, TB; Sinoie, Grindul Lupilor, 27.VI.2017, leg. KI, KJ, PA, TB, 5.VI.2018, leg. FK, KI, KJ, PA, 22.VI.2019, leg. KI, KJ, PA, TB; Crucea, 9.VI.2018, leg. FK, KI, KJ, PA.
Pseudostenapion simum (GERMar, 1817)

New data: Tulcea County: Slava Rusă, 9.V.2017, leg. FK, KI, KJ, PA.

Aspidapiina Alonso-ZaraZAGa, 1990

Alocentron (Alocentron) curvirostre (GYLLENHAL, 1833)

Published data: Montandon 1908, WaGner 1910

New data: Tulcea County: Slava Cercheză, Păd. Babadag, Fântâna Țiganului, 18.VIII.1992, leg. AP (Coll. Mus. G. AnTIPA).

Aspidapion (Aspidapion) radiolus (MARSHAM, 1802) Published data: Fleck 1905, Montandon 1908, Negru and RoşCa 1967, Raduta 2014

New data: Tulcea County: Gorgova, 16.VI.1993, leg. TLA; Pădurea Letea (forest), 17.VI.1993, leg. TLA; Greci (village), 7-8.V.2017, leg. FK, KI, KJ, PA, 22.VI.2017, leg. KI; Constanţa County: Gura Dobrogei, 30.IV-2.V.2014, leg. KI, IZ, PA, SzL; Albeşti, Pădurea Hagieni (forest), 12.V.2017, leg. FK, KI, KJ, PA; Limanu, 12.V.2017, leg. FK, KI, KJ, PA; 23 August, 13.V.2017, leg. FK, KI, KJ, PA; Hagieni, Pădurea Hagieni (forest), 13.V.2017, leg. FK, KI, KJ, PA.

Aspidapion (Aspidapion) validum (GERMAR, 1817) Published data: MONTANDON 1908, WAGNER 1910, TeOdor and Manole 1996, Raduta 2014

New data: Tulcea County: M-ţii Măcin, Mănăstirea Cocoş, 26.VII.1958; Periprava, 5-8.IX.1964., leg. NS; Maliuc, Mila 26, 8.VIII.1991, leg. IM (Coll. Mus. G. ANTIPA) - Periprava, 1.VII.2005, leg. DDC; Greci (village), 22.VI.2017, leg. KI; Constanța County: Băneasa, Canaraua Fetii, 20.VII.1958. leg IS (Coll. Mus. G. AnTIPA).

Aspidapion (Koestlinia) aeneum (FABRICIUS, 1775) Published data: IENIŞTEA 1968

New data: Tulcea County: M-ţii Măcin, to the Peak Ţuţuiatu, 28.IV.2010, leg. TLA; Constanţa County: Hagieni, Pădurea Hagieni (forest), 13.V.2017, leg. FK, KI, KJ, PA.

Catapiina Alonso-Zarazaga, 1990

Catapion jaffense (DESBRoChers des Loges, 1895) New data: Tulcea County: Enisala (wooded pasture), 13.IX.2016, leg. KI, KJ, PA, TB; Babadag (forest), 13.IX.2016, leg. KI, KJ, PA, TB.

Catapion meieri (DESBROCHERS des LOGES, 1895) New data: Tulcea County: Nifon, pârâul Pârlita valley, 23.VI.2017, leg. KI.

\section{Catapion pubescens (KIRBY, 1811)}

Published data: Montandon 1887, Fleck 1905, WaGner 1910, Negru and Roşca 1967, Raduta 2014 New data: Tulcea County: N from Nifon, 23.VI.2017, leg. KI, KJ, PA, TB; Constanţa County: Esechioi 
(forest), 7.VII.2014, leg. KI, OA, PA, SzL, Esechioi (semi-dry steppic grassland on loess), 16.IX.2016, leg. KI, KJ, PA, TB; Esechioi, Lacul Bugeac, towards the south Lake Bugeac, 16.IX.2016, leg. KI, KJ, PA, TB.

Catapion seniculus (KIRBY, 1808)

Published data: Montandon 1887, Fleck 1905

New data: Tulcea County: M-ţii Măcin, Valea Fagilor (dried lake bed) 30.V.2005, leg. CsS; Sulina, 4.VII.2005, leg. DDC; Atmagea env., 24.IX.2010, leg. JK; Babadag (forest), 13.IX.2016, leg. KI, KJ, PA, TB; Smârdan, Braţul Măcin, 22.VI.2017, leg. KI, KJ, PA, TB; Nifon, pârâul Pârlita valley, 23.VI.2017, leg. KI; Constanţa County: Băneasa, Canaraua Fetii (quarry), 7.VII.2014, leg. KI, OA, PA, SzL; Esechioi (forest), 7.VII.2014, leg. KI, OA, PA, SzL; Ostrov, Lacul Bugeac, 16.IX.2016, leg. TB.

Ceratapiina Alonso-Zarazaga, 1990

Ceratapion (Acanephodus) onopordi onopordi (KIRBY, 1808)

Published data: Montandon 1908, Wagner 1910

New data: Tulcea County: Valea Teilor, 22.V.1997, leg. CP, CH (Coll. Mus. G. Antipa) - Pădurea Letea (forest), 17.VI.1993, leg. TLA; M-ţii Măcin, Pricopan, 1.VI.2005, leg. CsS; Atmagea, 28.IV.2012, leg. KI; Slava Rusă, 24-25.V.2013, leg. KI, PA, RI, SzL, 20.V.2015, leg. PA, RI, SzK, SzL; Enisala (castle), 13.IX.2016, leg. KI, KJ, PA, TB; Nifon, pârâul Pârlita valley, 23.VI.2017, leg. KI, KJ, PA, TB; $2 \mathrm{~km}$ from Slava Rusă to NE, 3.VI.2018, leg. FK, KI, KJ, PA; Constanţa County: Furnica, Rez. Dumbrăveni, 1314.V.1995, leg. CP (Coll. Mus. G. Antipa) - Vadu env., Grindul Chituc, 21.IX.2010, leg. JK; Gura Dobrogei, 26.V.2013, leg. KI, PA, RI, SzL; Balta Limanu, 5-6. VII.2014, leg. KI, OA, PA, SzL; Pădurea Hagieni (forest), 6.VII.2014, leg. KI, OA, PA, SzL; Albeşti, Pădurea Hagieni (forest), 12.V.2017, leg. FK, KI, KJ, PA; Istria, Cetatea Histria, 21.V.2015, leg. PA, RI, SzK, SzL; Palazu Mic, 22.V.2015, leg. PA, RI, SzK, SzL; Murfatlar, Fântâniţa, 22.V.2015, leg. PA, RI, SzK, SzL; Negureni, Pădurea Valea Cişmelelor, 23.V.2015, leg. PA, RI, SzK, SzL; Negru Vodă (Nature Reserve) 25.IX.2015, leg. KI, KJ, PA; Băneasa, Canaraua Fetii (quarry), 2-3.IV.2016, leg. FK, KI, PA, SzL; Băneasa, Canaraua Fetii (forest), 15.IX.2016 and 17, leg. KI, KJ, PA, TB; Esechioi (semi-dry slope steppic grassland with shrub encroachment), 15.IX.2016, leg. KI, KJ, PA, TB; Ostrov, Lacul Bugeac, 16.IX.2016, leg. KI, KJ, PA, TB; 23 August, 13.V.2017, leg. FK, KI, KJ, PA; Limanu, 12.V.2017, leg. FK, KI, KJ, PA; Hagieni, Pădurea Hagieni (forest), 13.V.2017, leg. FK, KI, KJ, PA; Sinoie, Grindul Lupilor, 27.VI.2017, leg. KI, KJ, PA, TB, 5.VI.2018, leg. FK, KI, KJ, PA.

Ceratapion (Angustapion) beckeri (DESBROCHERS des Loges, 1875)

Published data: Wagner 1910, Altea and Osella 1991, WANAT 1995
New data: Constanţa County: Esechioi (forest), 7.VII.2014, leg. KI, OA, PA, SzL.

Ceratapion (Angustapion) cylindricolle (GYLLENHAL, 1839)

Published data: WANAT 1995

New data: Tulcea County: Codru Monastery 22.IX.2015, leg. KI, KJ, PA; Enisala (castle) 22.IX.2015, leg. KI, KJ, PA; Atmagea env., 24.IX.2010, leg. JK, KP; $1,5 \mathrm{~km}$ from Beidaud to SW, 25.VI.2017, leg. KI, KJ, PA, TB; Jurilovca, 23.VI.2019, leg. PA; Constanţa County: Hârşova, leg. ALM (Coll. Mus. G. ANTIPA) - Istria, Cetatea Histria, 14.IX.2016, leg. KI, KJ, PA, TB.

Ceratapion (Angustapion) decolor (DESBROCHERS des LOGES, 1875)

Published data: WANAT 1995

New data: Constanţa County:Esechioi (forest), 7.VII.2014, leg. KI, OA, PA, SzL.

Ceratapion (Angustapion) macrorrhynchum (Eppelsheim, 1888)

New data: Tulcea County: Enisala (castle) 22.IX.2015, leg. KI, KJ, PA; Codru Monastery, 24.VI.2017, leg. KI.

Ceratapion (Ceratapion) carduorum (KIRBY, 1808)

Published data: MonTANDON 1887, 1908; FleCK 1905; WAGNER 1910

New data: Tulcea County: M-ţii Măcin, to the Peak Ţuţuiatu, 28.IV.2010, leg. TLA.

Ceratapion (Ceratapion) gibbirostre (GYLLENHAL, 1813) Published data: WANAT 1995

New data: Tulcea County: Slava Rusă, 24-25.V.2013, leg. KI, PA, RI, SzL; Codru Monastery, 10-11.V.2017, leg. FK, KI, KJ, PA, 24-25.VI.2017, leg. KI, KJ, PA, TB; Nifon, pârâul Pârlita valley, 23.VI.2017, leg. KI, KJ, PA, TB; Enisala, Cetatea Heraclea, 25.VI.2017, leg. KI; 2 km from Slava Rusă to NE, 3.VI.2018, leg. FK, KI, KJ, PA; Enisala (wooded pasture), 4.VI.2018, leg. FK, KI, KJ, PA; Constanţa County: Furnica, Rez. Dumbrăveni, 13-14.V.1995, leg. CP (Coll. Mus. G. ANTIPA) - Gura Dobrogei, 26.V.2013, leg. KI, PA, RI, SzL; Balta Limanu, 5-6.VII.2014, leg. KI, OA, PA, SzL; Murfatlar, Fântâniţa, 24-25.IX.2015, leg. KI, KJ, PA, 14.IX.2016, leg. KI, KJ, PA, TB; Băneasa, Canaraua Fetii (forest), 17.IX.2016, leg. KI, KJ, PA, TB; Hagieni, Pădurea Hagieni (forest), 13.V.2017, leg. FK, KI, KJ, PA; $2 \mathrm{~km}$ from Sinoie to SE, 27.VI.2017, leg. KI, KJ, PA, TB.

Ceratapion (Clementiellus) orientale (GERSTÄCKER, 1854)

Published data: Wagner 1910, Teodor and Manole 1996, RAduTA 2014

New data: Tulcea County: M-ţii Măcin, Greci, 18-19. VI.2019, leg. KI, KJ, PA, TB. 
Ceratapion (Echinostroma) basicorne (Illiger, 1807)

Published data: MonTANDON 1887, 1908; FleCK 1905; WAGNER 1910

New data: Constanţa County: Esechioi (forest), 7.VII.2014, leg. KI, OA, PA, SzL.

\section{Ceratapion (Echinostroma) penetrans penetrans} (GERMAR, 1817)

Published data: JAQUET 1902a, FLeCK 1905, WAGNER 1910

New data: Tulcea County: Periprava, 13.X.1966, leg. ŞN; Pădurea Caraorman, 24.VIII.1992, leg. AP; Pădurea Letea, 22.IX.2010, leg. IŞI (Coll. Mus. G. ANTIPA) - Babadag (forest), 2.VI.2005, leg. CsS; Parcheş 23.IX.2015, leg. KI, KJ, PA; Enisala, Cetatea Heraclea, 25.VI.2017, leg. KI, KJ, PA, TB; M-ţii Măcin, Greci, 18-19.VI.2019, leg. KI, KJ, PA, TB; Enisala (castle), 20.VI.2019, leg. KI, KJ, PA, TB; Jurilovca, 23.VI.2019, leg. KI, KJ, PA, TB; Constanţa County: Sinoie, Grindul Lupilor, 27.VI.2017, leg. KI, KJ, PA, TB, 5.VI.2018, leg. FK, KI, KJ, PA, 22.VI.2019, leg. KI, KJ, PA, TB; Vadu, Grindul Chituc, 22.VI.2019, leg. KI, KJ, PA, TB.

*Ceratapion (Echinostroma) scalptum caviceps (Desbrochers des Loges, 1870)

New data: Tulcea County: Smârdan, Braţul Măcin, 18.VI.2019, leg. KI.

\section{Diplapion confluens (KIRBY, 1808)}

Published data: Montandon 1887, 1908; FleCK 1905; NEgRU and RoşCA 1967; RADUTA 2014

New data: Tulcea County: Smârdan, Braţul Măcin, 2.VI.2018, leg. FK, KI, KJ PA; Constanţa County: 2 $\mathrm{km}$ from Sinoie to SE, 27.VI.2017, leg. KI, 5.VI.2018, leg. FK, KI, KJ, PA; Istria, Cetatea Histria, 26-28. VI.2017, leg. KI; Gura Dobrogei, 7.VI.2018, leg. FK, KI, KJ, PA.

\section{Diplapion detritum (Mulsant \& REY, 1858)}

Published data: MontANDON 1908, WAGNER 1910, WANAT 1995

New data: Tulcea County: Enisala (castle), 10.V.2017, leg. FK, KI, KJ, PA; Nifon, pârâul Pârlita valley, 23.VI.2017, leg. KI, KJ, PA, TB; Enisala, Cetatea Heraclea, 25.VI.2017, leg. KI, KJ, PA, TB; Constanţa County: Grădina env., 19.IX.2010, leg. JK; Pecineaga (forest), 14.V.2017, leg. FK, KI, KJ, PA; Amzacea, 14.V.2017, leg. FK, KI, KJ, PA.

Diplapion sareptanum (DESBRochers des Loges, 1867)

Published data: Montandon 1908, WaGner 1910

New data: Constanţa County: Hârşova, leg. ALM (Coll. Mus. G. AntIPA).

\section{Diplapion stolidum (GERMAR, 1817)}

New data: Tulcea County: Smârdan, Braţul Măcin, 28.VI.2017, leg. KI, KJ, PA, TB, 2.VI.2018, leg.
FK, KI, KJ, PA, 18.VI.2019, leg. KI, KJ, PA, TB; Enisala, 20.VI.2019, leg. KI; Constanţa County: Murfatlar, Fântâniţa, 24-25.IX.2015, leg. KI, KJ, PA; Agigea, 25.IX.2015, leg. KI; Hagieni, Pădurea Hagieni (forest), 13.V.2017, leg. FK, KI, KJ, PA; Gura Dobrogei, 27.VI.2017, leg. KI, KJ, PA, TB; 2 $\mathrm{km}$ from Sinoie to SE, 27.VI.2017, leg. KI, KJ, PA, TB, 5.VI.2018, leg. FK, KI, KJ, PA; Sinoie, Grindul Lupilor, 27.VI.2017, leg. KI, KJ, PA, TB; Istria, Cetatea Histria, 26-28.VI.2017, leg. KI, 6.VI.2018, leg. KI; Vadu, Grindul Chituc, 6.VI.2018, leg. FK, KI, KJ, PA.

\section{Omphalapion hookerorum (KIRBY, 1808) (= hookeri KIRBY, 1808) \\ New data: Tulcea County: Nifon, pârâul Pârlita valley, 23.VI.2017, leg. KI, KJ, PA, TB.}

\section{Taphrotopium (Taphrotopium) sulcifrons (HERBST, 1797) \\ Published data: Teodor and Manole 1996, Raduta 2014}

New data: Tulcea County: Enisala (wooded pasture), 20.VI.2019, leg. KI; Constanţa County: Istria env., Cetatea Histria, 19.IX.2010, leg. JK, KP, 26, 28.VI.2017, leg. KI, KJ, PA, TB, 6.VI.2018, leg. FK, KI, KJ, PA, 21.VI.2019, leg. KI, KJ, PA, TB; 2 km from Sinoie to SE, 27.VI.2017, leg. KI, KJ, PA, TB, 21, 23.VI.2019, leg. KI, KJ, PA, TB; Sinoie, Grindul Lupilor, 27.VI.2017, leg. TB; Vadu, Grindul Chituc, 22.VI.2019, leg. KI.

Ixapiina Alonso-ZARAZAGA, 1990

Trichopterapion holosericeum (GYLLENHAL, 1833)

Published data: Fleck 1905, Montandon 1908, WAGNER 1910

New data: Tulcea County: Beştepe, 13-14.VI.1993, leg. IM; Celic Dere, 24.V.1997, leg. CP (Coll. Mus. G. ANTIPA) - Horia, Praporgescu, 23.IV.2009, leg. TLA; Codru env., 3.V.2009, leg. JK; M-ţii Măcin, to the Peak Ţuţuiatu, 28.IV.2010, leg. TLA; Atmagea, 28.IV.2012, leg. KI; Babadag (forest), 25.V.2013, leg. KI, 22.IX.2015, leg. KI, KJ, PA; Enisala (wooded pasture), 24-25.V.2013, leg. KI, PA, RI, SzL, 29.IV3.V.2014, leg. KI, IZ, PA, SzL, 13.IX.2016, leg. KI, KJ, PA, TB; Codru Monastery, 23-25.V.2013, leg. KI, PA, RI, SzL, 22.IX.2015, leg. KI, KJ, PA; Slava Rusă, 23.IV.2009, leg. TLA, 1-3.V.2014, leg. KI, IZ, PA, SzL, 20.V.2015, leg. PA, RI, SzK, SzL, 23.IX.2015, leg. KI, KJ, PA, 31.III.2016, leg. FK, KI, PA, SzL, 9.V.2017, leg. FK, KI, KJ, PA, 11.V.2017, leg. FK, KI, KJ, PA; Enisala (castle) 22.IX.2015, leg. KI, KJ, PA, 10.V.2017, leg. FK, KI, KJ, PA, 20.VI.2019, leg. KI; M-ţii Măcin, Greci 7.V.2017, leg. FK, KI, KJ, PA; M-ţii Măcin, 5 km S Greci, 8.V.2017, leg. FK, KI, KJ, PA; 2 km from Slava Rusă to NE, 3.VI.2018, leg. KI; Constanţa County: Furnica, Rez. Dumbrăveni, 13-14.V.1995, leg. CP, (Coll. Mus. G. ANTIPA) - Gura Dobrogei, 26.V.2013, leg. KI, PA, RI, SzL; Murfatlar, 
Fântâniţa，4-5.VII.2014， leg. KI，OA，PA，SzL; Esechioi (forest), 1-2.IV.2016, leg. FK, KI, PA, SzL; Băneasa, Canaraua Fetii (quarry), 2-3.IV.2016, leg. FK, KI, PA, SzL.

Kalcapiina Alonso-ZaraZaga, 1990

Kalcapion pallipes (KIRBY, 1808)

New data: Tulcea County: Enisala (castle), 21.V.2015, leg. PA, RI, SzK, SzL; Slava Rusă, 31.III.2016, leg. FK, KI, PA, SzL, 11.V.2017, leg. FK, KI, KJ, PA; Codru Monastery, 30-31.III.2016, leg. FK, KI, PA. SzL; Slava Rusă, Uspenia Monastery, 11.V.2017, leg. FK, KI, KJ, PA; $2,5 \mathrm{~km}$ from Stejaru to NW, 25.VI.2017, leg. KI, KJ, PA, TB; General Praporgescu, 3.VI.2018, leg. FK, KI, KJ, PA; Constanţa County: Murfatlar, Fântâniţa, 4-5.VII.2014, leg. KI, OA, PA, SzL; Gura Dobrogei, 1.IV.2016, leg. FK, KI, PA, SzL; Esechioi (forest), 1-2.IV.2016, leg. FK, KI, PA, SzL.

\section{Kalcapion semivittatum (GYLLENHAL, 1833) \\ Published data: WAGNER 1910}

Melanapion (Melanapion) minimum (HERBST, 1797) Published data: Montandon 1887, 1908; FleCK 1905; TeOdOR and Manole 1996; Raduta 2014

New data: Constanţa County: Furnica, Rez. Dumbrăveni, 13-14.V.1995, leg. CP (Coll. Mus. G. ANTIPA).

\section{Squamapion atomarium (KIRBY, 1808)}

Published data: Montandon 1887; Fleck 1905

New data: Tulcea County: Atmagea env., 24.IX.2010, leg. JK; N from Nifon, 23.VI.2017, leg. KI, KJ, PA, TB; $3 \mathrm{~km}$ from Slava Rusă to NE, 24.VI.2017, leg. KI, KJ, PA, TB; S from Hamcearca, 24.VI.2017, leg. KI, KJ, PA, TB.

\section{Squamapion flavimanum (GYLLENHAL, 1833)}

Published data: MonTANDOn 1908, WAGNer 1910

New data: Tulcea County: „Dobroudja, Măcin-Greč̄, A. L. MONTANDON", flavimanum v. torquatum WeNCK. WAGNER det." Coll. PA.

\section{Squamapion vicinum (KIRBY, 1808)}

Published data: Montandon 1908, Wagner 1910 New data: Constanţa County: Ostrov, Lacul Bugeac, 16.IX.2016, leg. KI.

\section{Taeniapion rufulum (WENCKER, 1864)}

Published data: MonTANDON 1908, Wagner 1910

New data: Tulcea County: M-ţii Măcin, 5 km S Greci, 8.V.2017, leg. FK, KI, KJ, PA.

Taeniapion urticarium urticarium (HERBST, 1784) Published data: MonTANDON 1908, WAGNER 1910 New data: Tulcea County: Luncaviţa, Valea Fagilor, 23.V.1997, leg. CH, (Coll. Mus. G. ANTIPA) Agighiol, 1.V.1993, 12.VI.1993, leg. TLA; Atmagea, 23.IV.2009, leg. TLA; M-ţii Măcin, to the Peak
Ţuţuiatu, 28.IV.2010, leg. TLA; Atmagea, 28.IV.2012, leg. KI; Slava Rusă, 24-25.V.2013, leg. KI, PA, RI, SzL, 20.V.2015, leg. PA, RI, SzK, SzL, 9.V.2017, leg. FK, KI, KJ, PA, 11.V.2017, leg. FK, KI, KJ, PA; Babadag (forest), 13.IX.2016, leg. KI, KJ, PA, TB; Slava Rusă, Uspenia Monastery, 11.V.2017, leg. FK, KI, KJ, PA; Nifon, pârâul Pârlita valley, 23.VI.2017, leg. KI, KJ, PA, TB; 2,5 km from Stejaru to NW, 25.VI.2017, leg. KI, KJ, PA, TB; Horea, 3.VI.2018, leg. KI; Enisala, 20.VI.2019, leg. KI; Constanţa County: Furnica, Rez. Dumbrăveni, 13-14.V.1995, leg. CP; Negureni, 25.VI.1995, leg. CP, 21.VI.1996, leg. CP (Coll. Mus. G. AntiPA) - Gura Dobrogei, 2829.IV.2012, leg. KI, 26.V.2013, leg. KI, PA, RI, SzL, 1.IV.2016, leg. FK, KI, PA, SzL, 12.V.2017, leg. FK, KI, KJ, PA, 7.VI.2018, leg. FK, KI, KJ, PA; Băneasa, Canaraua Fetii (quarry), 2-3.IV.2016, leg. FK, KI, PA, SzL; Băneasa, Canaraua Fetii (forest), 15-17. IX.2016, leg. KI, KJ, PA, TB, 17.IX.2016, leg. KI, KJ, PA, TB; Esechioi (semi-dry slope steppic grassland with shrub encroachment), 15.IX.2016, leg. KI, KJ, PA, TB; Ostrov, Lacul Bugeac, 16.IX.2016, leg. KI, KJ, PA, TB; Sălcioara, Lacul Sălcioara, 13.IX.2016, leg. KI, KJ, PA, TB; Hagieni, Pădurea Hagieni (forest), 13.V.2017, leg. FK, KI, KJ, PA; Pecineaga (forest), 14.V.2017, leg. FK, KI, KJ, PA; Murfatlar, Fântâniţa, 7-8.VI.2018, leg. FK, KI, KJ, PA.

Malvapiina Alonso-Zarazaga, 1990

Malvapion malvae (FABRICIUS, 1775)

Published data: Montandon 1908, Wagner 1910, TeOdOR and Manole 1996, Raduta 2014

New data: Tulcea County: Frecăţei, Celic Dere Monastery (hillside), 31.V.2005, leg. CsS; M-ţii Măcin, Greci, 8.V.2017, leg. FK, KI, KJ, PA.

\section{Pseudapion fulvirostre (GYLLENHAL, 1833)}

Published data: Montandon 1908, WaGner 1910, TEOdor and Manole 1996, Raduta 2014

New data: Tulcea County: Letea (forest), 12.VII.1962, leg. APG; Periprava, 15-18.VII.1964, leg. XS; Letea, C. A. Rosetii, 20.VII.1964, leg. ŞN; Maliuc, Mila 26, 7.VIII.1991, leg. IM, 8.VIII.1991, leg. IM, 9.VIII.1991, leg. RS; Dunavăţ, 31.V.1996, leg. CH (Coll. Mus. G. ANTIPA) - Canalul Magearu, 17.VI.1993, leg. TLA; Gorgova, 16.VI.1993, leg. TLA; Codru Monastery, 23-25.V.2013, leg. KI, PA, RI, SzL; Slava Rusă, 2425.V.2013, leg. KI, PA, RI, SzL, 1-3.V.2014, leg. KI, IZ, PA, SzL; Smârdan, Braţul Măcin, 22.VI.2017, leg. KI, KJ, PA, TB; Jijila, râul Jijila, 23.VI.2017, leg. KI, KJ, PA, TB; 2 km from Slava Rusă to NE, 3.VI.2018, leg. KI; Enisala, 20.VI.2019, leg. KI; Constanţa County: Negureni, 21.VI.1996, leg. CP (Coll. Mus. G. ANTIPA) - Murfatlar, Fântâniţa, 4-5.VII.2014, leg. KI, OA, PA, SzL, 24-25.IX.2015, leg. KI, KJ, PA, 7-8.VI.2018, leg. FK, KI, KJ, PA; Pădurea Hagieni (forest), 6.VII.2014, leg. KI, OA, PA, SzL; Băneasa, Canaraua Fetii, 23.V.2015, leg. PA, RI, SzK, SzL. 
Pseudapion rufirostre (FABRICIUS, 1775)

Published data: JAQUET 1901, Fleck 1905, WAGNER 1910

New data: Tulcea County: M-ții Măcin, Greci, 7.V.2017, leg. FK, KI, KJ, PA; Smârdan, Braţul Măcin, , 22.VI.2017, leg. KI, KJ, PA, TB; S from Hamcearca, 24.VI.2017, leg. KI; Constanţa County: Băneasa, Canaraua Fetii (quarry), 2-3.IV.2016, leg. FK, KI, PA, SzL; Limanu, 12.V.2017, leg. FK, KI, KJ, PA; Albeşti, Pădurea Hagieni (forest), 12.V.2017, leg. FK, KI, KJ, PA; Hagieni, Pădurea Hagieni (forest), 13.V.2017, leg. FK, KI, KJ, PA; 23 August, 13.V.2017, leg. FK, KI, KJ, PA.

\section{Rhopalapion longirostre (OLIVIER, 1807)}

Published data: MonTANDON 1908

New data: Tulcea County: M-ţii Măcin, Pricopan, 1.VI.2005, leg. CsS; Slava Rusă, 24-25.V.2013, leg. KI, PA, RI, SzL, 1-3.V.2014, leg. KI, IZ, PA, SzL, 9.V.2017, leg. FK, KI, KJ, PA; Codru Monastery, 23-25.V.2013, leg. KI, PA, RI, SzL, 10-11.V.2017, leg. FK, KI, KJ, PA; M-ţii Măcin, Greci, 7.V.2017, leg. FK, KI, KJ, PA; Slava Rusă, Uspenia Monastery, 11.V.2017, leg. FK, KI, KJ, PA; S from Hamcearca, 24.VI.2017, leg. KI; Enisala, 20.VI.2019, leg. KI; Constanţa County: Băneasa, Canaraua Fetii (quarry), 7.VII.2014, leg. KI, OA, PA, SzL, 23.V.2015, leg. PA, RI, SzK, SzL; Oltina, 8.VII.2014, leg. KI, OA, PA, SzL; Murfatlar, Fântâniţa, 22.V.2015, leg. PA, RI, SzK, SzL, 24-25.IX.2015, leg. KI, KJ, PA; Esechioi (forest), leg. FK, KI, PA, SzL; Bugeac, 2.IV.2016, leg. FK, KI, PA, SzL; Vadu, Grindul Chituc, 6.VI.2018, leg. KI.

Metapiina Alonso-ZaraZaga, 1990

*Metapion ermischi (Voss, 1969) (fig. 5)

New data: Tulcea County: Braţul Sfầntu Gheorghe, 21-22.VI.2003, leg. TB; Babadag, (forest), 2.V.2005. leg. CsS, 25.V.2013, leg. KI; Codru Monastery, 23-25.V.2013, leg. KI, PA, RI, SzL, 19.V.2015, leg. PA, RI, SzK, SzL, 22.IX.2015, leg. KI, KJ, PA, 10-11.V.2017, leg. FK, KI, KJ, PA, 24-25.VI.2017, leg. KI, KJ, PA, TB, 20.VI.2019, leg. KI, KJ, PA, TB; M-ţii Măcin, Greci, 7.V.2017, leg. FK, KI, KJ, PA; Turcoaia, $6 \mathrm{~km}$ from Cerna to NW, 24.VI.2017, leg. KI, KJ, PA, TB; Jurilovca, 23.VI.2019, leg. KI, KJ, PA, TB; Constanţa County: Balta Limanu, 5-6. VII.2014, leg. KI, OA, PA, SzL; Limanu, 12.VI.2017, leg. FK, KI, KJ, PA.

\section{Metapion oculare (GYLLENHAL, 1833)}

Published data: WAGNER 1910, SCHUBERT 1956, ANGelov 1976, WANAT 1992

Oxystomatina Alonso-Zarazaga, 1990 Cyanapion (Cyanapion) columbinum (GERMAR, 1817)

New data: Tulcea County: N from Nifon, 23.VI.2017, leg. KI, KJ, PA, TB; Smârdan, Braţul Măcin, 22.VI.2017, leg. KI, KJ, PA, TB.

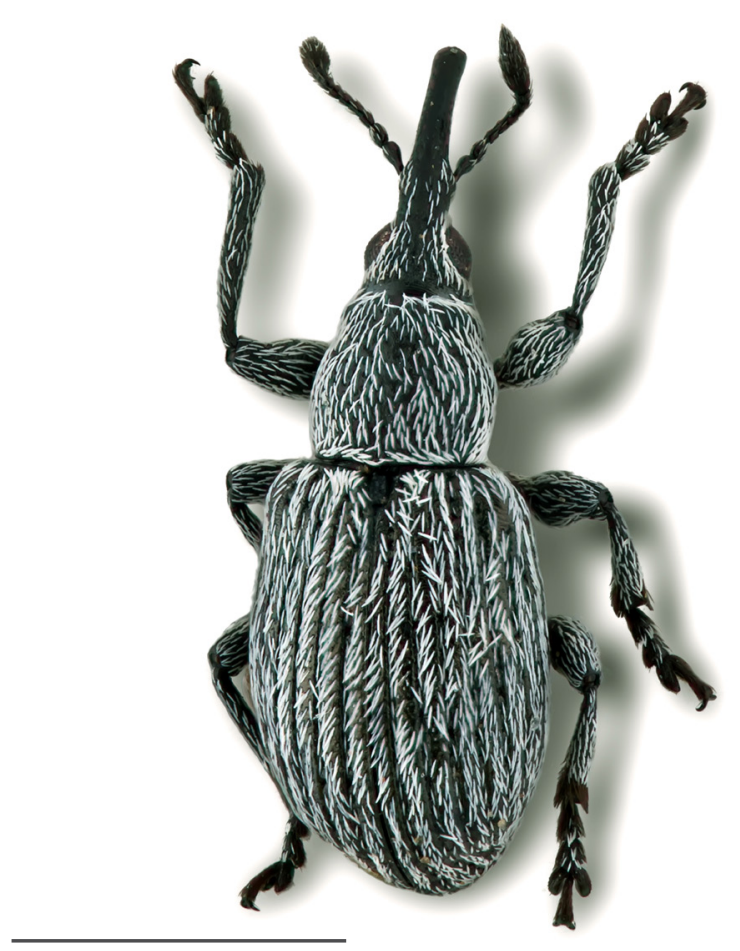

Fig. 5. Metapion ermischi (Voss, 1969). Scale bar: $1 \mathrm{~mm}$. Photo Zoltán CsATA

Eutrichapion (Cnemapion) gribodoi (DESBROCHERS des Loges, 1896)

Published data: Montandon 1908, WaGner 1910

Eutrichapion (Eutrichapion) ervi (KIRBY, 1808)

New data: Constanţa County: Băneasa, Canaraua Fetii (forest), 17.IX.2016, leg. TB.

Eutrichapion (Eutrichapion) viciae (PAYKULL, 1800) Published data: Montandon 1887, 1908; FleCK 1905; Wagner 1910; Negru and RoşCa 1967; RADUTA 2014

New data: Tulcea County: Slava Rusă, 31.III.2016, leg. FK, KI, PA, SzL; Constanţa County: Murfatlar, Fântâniţa，4-5.VII.2014, leg. KI，OA，PA， SzL; Esechioi (forest), 7.VII.2014, leg. KI, OA, PA, SzL; Istria, Cetatea Histria, 14.IX.2016, leg. KI, KJ, PA, TB; Băneasa, Canaraua Fetii (forest), 17.IX.2016, leg. KI, KJ, PA, TB.

\section{Eutrichapion (Phalacrolobus) melancholicum} (WENCKER, 1864)

New data: Tulcea County: Nifon, pârâul Pârlita valley, 23.VI.2017, leg. KI, KJ, PA, TB.

Eutrichapion (Psilocalymma) punctiger (PAYKULL, 1792)

Published data: Montandon 1908

New data: Constanţa County: Băneasa, Canaraua Fetii, 23.V.2015, leg. PA, RI, SzK, SzL.

Hemitrichapion (Dimesomyops) pavidum (GERMAR, 1817) 
New data: Constanţa County: Băneasa, Canaraua Fetii (quarry), 2-3.IV.2016, leg. FK, KI, PA, SzL; Băneasa, Canaraua Fetii (forest), 17.IX.2016, leg. KI, KJ, PA, TB.

Holotrichapion (Apions) pisi (FABRICIUS, 1801) New data: Tulcea County: M-ţii Măcin, Valea lui Arsu, 5.X.2007, leg. CI (Coll. Mus. R. Angheluță); Măcin, Valea Suluc, 27.V.2005, leg. IM (Coll. Mus. G. ANTIPA) - Enisala, 29.IV-3.V.2014, leg. KI, IZ, PA, SzL; Codru Monastery, 19.V.2015, leg. PA, RI, SzK, SzL; Nifon, pârâul Pârlita valley, 23.VI.2017, leg. KI, KJ, PA, TB; $2,5 \mathrm{~km}$ from Stejaru to NW, 25.VI.2017, leg. KI, KJ, PA, TB; 2,7 km from Horia to S, 28.VI.2017, leg. KI, KJ, PA, TB; Smârdan, Braţul Măcin, 28.VI.2017, leg. KI, KJ, PA, TB, 2.VI.2018, leg. FK, KI, KJ, PA; Constanţa County: Negureni, 17.V.1994, leg. AP (Coll. Mus. G. ANTIPA) - Murfatlar, Fântâniţa, 4-5.VII.2014, leg. KI, OA, PA, SzL; Ion Corvin, 15.IX.2016, leg. KI, KJ, PA, TB; Băneasa, Canaraua Fetii (forest), 15-17. IX.2016, leg. KI, KJ, PA, TB; Ostrov, Lacul Bugeac, northward Lake Bugeac, 16.IX.2016, leg. KI, KJ, PA, TB; Esechioi, Lacul Bugeac, towards the south Lake Bugeac, 16.IX.2016, leg. KI, KJ, PA, TB; Pecineaga (forest), 14.V.2017, leg. KI; Hagieni, Pădurea Hagieni (forest), 13.V.2017, leg. KI; Albeşti, Pădurea Hagieni (forest), 12.V.2017, leg. KI.

Holotrichapion (Apions) pullum (GYLLENHAL, 1833) (=Apion aestimatum FAUST, 1891)

New data: Tulcea County: M-ţii Măcin, Valea Fagilor (quarry) 30.V.2005, leg. CsS; Frecăţei, Celic Dere Monastery (hillside), 31.V.2005, leg. CsS; M-ţii Măcin, Pricopan, 1.VI.2005, leg. CsS; Codru env., 3.V.2009, leg. JK; Atmagea env., 24.IX.2010, leg. JK; Codru Monastery, 23-25.V.2013, leg. KI, PA, RI, SzL, 30.IV-3.V.2014, leg. KI, IZ, PA, SzL, 19.V.2015, leg. PA, RI, SzK, SzL, 10-11.V.2017, leg. FK, KI, KJ, PA; Babadag (forest), 30.IV.2014, leg. KI, IZ, PA, SzL; Enisala (wooded pasture), 29.IV-3.V.2014, leg. KI, IZ, PA, SzL, 13.IX.2016, leg. KI, KJ, PA, TB; Slava Rusă, 20.V.2015, leg. PA, RI, SzK, SzL, 9.V.2017, leg. FK, KI, KJ, PA, 11.V.2017, leg. FK, KI, KJ, PA; Enisala (castle), 21.V.2015, leg. PA, RI, SzK, SzL, 22.IX.2015, leg. KI, KJ, PA, 10.V.2017, leg. FK, KI, KJ, PA; Parcheş, 23.IX.2015, leg. KI, KJ, PA; Greci (village), 8.V.2017, leg. FK, KI, KJ, PA; Smârdan, Braţul Măcin, 7.V.2017, leg. FK, KI, KJ, PA, 22.VI.2017, leg. KI, KJ, PA, TB, 2.VI.2018, leg. FK, KI, KJ, PA; Slava Rusă, Uspenia Monastery, 11.V.2017, leg. FK, KI, KJ, PA; M-ţii Măcin, Greci, 7.V.2017, leg. FK, KI, KJ, PA; 2,7 km from Horia to S, 28.VI.2017, leg. KI, KJ, PA, TB; Ciucurova, 3.VI.2018, leg. FK, KI, KJ, PA; 2 km from Slava Rusă to NE, 3.VI.2018, leg. FK, KI, KJ, PA; Turcoaia, 6 $\mathrm{km}$ from Cerna to NW, 3.VI.2018, leg. KI; Constanţa County: Tariverde env., 19.IX.2010, leg. JK; Vadu env., Grindul Chituc, 21.IX.2010, leg. JK; Cheia env.,
Cheia Nature Reserve., 19.IX.2010, leg. JK, 2.V.2014, leg. KI, IZ, PA, SzL; Gura Dobrogei, 28.IV.2012, leg. KI, 26.V.2013, leg. KI, PA, RI, SzL, 30.IV-2.V.2014, leg. KI, IZ, PA, SzL, 1.IV.2016, leg. FK, KI, PA, SzL, 12.V.2017, leg. FK, KI, KJ, PA, 25-27.VI.2017, leg. KI, KJ, PA, TB; Murfatlar, Fântâniţa, 4-5.VII.2014, leg. KI, OA, PA, SzL, 22.V.2015, leg. PA, RI, SzK, SzL, 24-25.IX.2015, leg. KI, KJ, PA; 5 km SW Gura Dobrogei, above Peştera "La Adam", SW end of gorge, 1.VI.2016, leg. MGy; Pădurea Hagieni (forest), 6.VII.2014, leg. KI, OA, PA, SzL; Histria, 21.V.2015, leg. PA, RI, SzK, SzL; Palazu Mic, 22.V.2015, leg. PA, RI, SzK, SzL; Negru Vodă (Nature Reserve), 25.IX.2015, leg. KI, KJ, PA; Agigea, 25.IX.2015, leg. KI; Ostrov, 2.IV.2016, leg. FK, KI, PA, SzL; Băneasa, Canaraua Fetii (forest), 15-17.IX.2016, leg. KI, KJ, PA, TB; Esechioi (forest), 16.IX.2016, leg. KI, KJ, PA, TB; Ostrov, Lacul Bugeac, 16.IX.2016, leg. KI, KJ, PA, TB; Ion Corvin, 15.IX.2016, leg. KI, KJ, PA, TB; Limanu, 12.V.2017, leg. FK, KI, KJ, PA; Pecineaga (forest), 14.V.2017, leg. FK, KI, KJ, PA.

\section{Holotrichapion (Holotrichapion) ononis (KIRBY, 1808)}

Published data: WAGNER 1910

\section{Ischnopterapion}

(Chlorapion)

cognatum

(Hochнuth, 1851)

Published data: WAGNER 1910

New data: Constanţa County: Mangalia, leg. ALM (Coll. Mus. G. ANTIPA) - Murfatlar, Fântâniţa, 4-5. VII.2014, leg. KI, OA, PA, SzL, 22.V.2015, leg. PA, RI, SzK, SzL; Gura Dobrogei, 1.IV.2016, leg. FK, KI, PA. SzL, 25-27.VI.2017, leg. KI, 7.VI.2018, leg. FK, KI, KJ, PA.

Ischnopterapion (Chlorapion) virens (HERBST, 1797) Published data: Montandon 1908, Negru and RoşCa 1967, RAduta 2014

New data: Tulcea County: $1.5 \mathrm{~km} \mathrm{~N}$ of Horia, Lacul Horia (lake), 24.IX.2010, leg. JK; Constanţa County: Cotu Văii, 23.VII.1997, leg. CP (Coll. Mus. G. ANTIPA).

Ischnopterapion (Ischnopterapion) fallens (MARSEUL, 1888)

New data: Constanţa County: Corbu env, 20.IX.2010, leg. JK.

Ischnopterapion (Ischnopterapion) loti (KIRBY, 1808) Published data: Wagner 1910, IENIŞTEA 1968

New data: Constanţa County: $2 \mathrm{~km}$ from Sinoie to SE, 27.VI.2017, leg. KI, KJ, PA, TB; Sinoie, Grindul Lupilor, 5.VI.2018, leg. FK, KI, KJ, PA, 22.VI.2019, leg. KI, KJ, PA, TB.

\section{Ischnopterapion}

(GERMAR, 1817)

(Ischnopterapion)

modestum

New data: Tulcea County: M-ţii Măcin (hillside, 200 
m), 29.V.2005, leg. CsS.

Mesotrichapion punctirostre (GYLLENHAL, 1839)

New data: Tulcea County: Babadag (forest), 13.IX.2016, leg. KI, KJ, PA, TB.

Oxystoma craccae (LINNÉ, 1767)

Published data: Montandon 1908, Wagner 1910

New data: Tulcea County: M-ţii Măcin, to the Peak Țuţuiatu, 28.IV.2010, leg. TLA; $1.5 \mathrm{~km} \mathrm{~N}$ of Horia, Lacul Horia (lake), 24.IX.2010, leg. JK; Slava Rusă, 1-3.V.2014, leg. KI, IZ, PA, SzL, 11.V.2017, leg. FK, KI, KJ, PA; N from Nifon, 23.VI.2017, leg. KI, KJ, PA, TB; 1,5 km from Beidaud to SW, 25.VI.2017, leg. KI, KJ, PA, TB; $2,7 \mathrm{~km}$ from Horia to S, 28.VI.2017, leg. KI, KJ, PA, TB; M-ţii Măcin, Greci, 18-19. VI.2019, leg. KI, KJ, PA, TB; Constanţa County: Esechioi (forest), 7.VII.2014, leg. KI, OA, PA, SzL.

Oxystoma dimidiatum (DESBROCHERS des LOGES, 1897) Published data: RADUTA 2014

Oxystoma ochropus (GERMAR, 1818)

Published data: WAGNER 1910

\section{Stenopterapion (Stenopterapion) intermedium} (EPPELSHEIM, 1875)

Published data: TeOdor and Manole 1996, Raduta 2014

New data: Constanţa County: Murfatlar, Fântâniţa, 7-8.VI.2018, leg. FK, KI, KJ, PA.

\section{Stenopterapion (Stenopterapion) meliloti (KIRBY, 1808)}

Published data: Montandon 1887, Fleck 1905

New data: Constanţa County: Istria, Cetatea Histria, 14.IX.2016, leg. KI, KJ, PA, TB; Sinoie, Grindul Lupilor, 22.VI.2019, leg. KI, KJ, PA, TB.

Stenopterapion (Stenopterapion) tenue (KIRBY, 1808) Published data: WAGNER 1910

New data: Tulcea County: M-ţii Măcin, Valea lui Moş Kalâm, 5.X.2007, leg. CI (Coll. Mus. R. ANGHELUȚǍ) - Babadag (forest), 13.IX.2016, leg. KI, KJ, PA, TB; Smârdan, Braţul Măcin, 28.VI.2017, leg. KI, KJ, PA, TB, 2.VI.2018, leg. FK, KI, KJ, PA; Nifon, pârâul Pârlita valley, 23.VI.2017, leg. KI, KJ, PA, TB; Ciucurova, 3.VI.2018, leg. FK, KI, KJ, PA; Enisala (castle), 20.VI.2019, leg. KI; Constanţa County: Esechioi (forest), 7.VII.2014, leg. KI, OA, PA, SzL; Ostrov, 2.IV.2016, leg. FK, KI, PA, SzL; Gura Dobrogei, 25.VI.2017, leg. KI, KJ, PA, TB, 7.VI.2018, leg. KI.

Piezotrachelina Voss, 1811

*Protapion angusticolle (Gyllenhal, 1833) (fig. 6)

New data: Constanţa County: Esechioi (forest), 7.VII.2014, leg. KI, OA, PA, SzL.

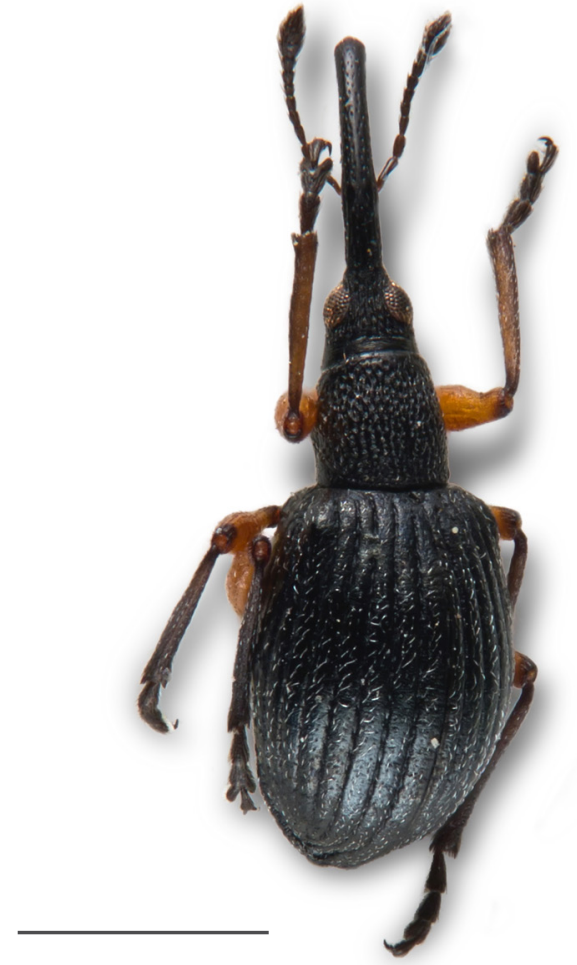

Fig. 6. Protapion angusticolle Gyllenhal, 1833. Scale bar: $1 \mathrm{~mm}$. Photo Zoltán CsatA

Protapion apricans (HERBST, 1797)

Published data: Montandon 1887

New data: Tulcea County: Frecăţei, Celic Dere Monastery (hillside) 31.V.2005, leg. CsS; Nifon, pârâul Pârlita valley, 23.VI.2017, leg. KI, KJ, PA, TB; Constanţa County: Băneasa, Canaraua Fetii (quarry), 7.VII.2014, leg. KI, OA, PA, SzL.

\section{Protapion assimile assimile (KIRBY,1808)}

New data: Tulcea County: Periprava, 1.VII.2005, leg. DDC; Babadag (forest), 13.IX.2016, leg. TB; Constanţa County: Cotu Văii, 23.VII.1997, leg. CP (Coll. Mus. G. Antipa).

\section{Protapion dissimile (Germar, 1817)}

New data: Tulcea County: M-ţii Măcin (hillside, 200 m), 29.V.2005, leg. CsS; Constanţa County: Esechioi (forest), 7.VII.2014, leg. KI, OA, PA, SzL.

\section{Protapion filirostre (KIRBY, 1808)}

Published data: IENIŞTEA 1968

\section{Protapion fulvipes fulvipes (GEOFFROY, 1785)}

Published data: MONTANDON 1887, 1908; JAQUET 1902a; WAGNER 1910

New data: Tulcea County: M-ţii Măcin, Valea Fagilor (dried lake bed), 30.V.2005, leg. CsS; M-ţii Măcin Luncaviţa, 30.V.2005, leg. CsS; Frecăţei, Celic Dere Monastery (hillside), 31.V.2005, leg. CsS; Atmagea env., 24.IX.2010, leg. JK; Smârdan, Braţul Măcin, 21 23.IX.2015, leg. KI, KJ, PA; Slava Rusă, 31.III.2016, leg. FK, KI, PA, SzL; Nifon, pârâul Pârlita valley, 23.VI.2017, leg. KI, KJ, PA, TB; S from Hamcearca, 
24.VI.2017, leg. KI, KJ, PA, TB; Constanţa County: Cotu Văii, 23.VII.1997, leg. CP(Coll. Mus. G. ANTIPA) - Esechioi (forest), 7.VII.2014, leg. KI, OA, PA, SzL; Istria, Cetatea Histria, 6.VI.2018, leg. FK, KI, KJ, PA.

\section{Protapion interjectum interjectum (DESBROCHERS} des Loges, 1895)

New data: Constanţa County: Băneasa, Canaraua Fetii (quarry), 7.VII.2014, leg. KI, OA, PA, SzL.

\section{Protapion nigritarse (KIRBY, 1808)}

Published data: MonTANDON 1908, WAGner 1910

New data: Tulcea County:Enisala(castle), 13.IX.2016, leg. KI, KJ, PA, TB; Slava Rusă, 9.V.2017, leg. FK, KI, KJ, PA, 11.V.2017, leg. FK, KI, KJ, PA; M-ţii Măcin, Greci, 7.V.2017, leg. FK, KI, KJ, PA; Greci (village), 8.V.2017, leg. FK, KI, KJ, PA; M-ţii Măcin, 5 km S Greci, 8.V.2017, leg. FK, KI, KJ, PA; Nifon, pârâul Pârlita valley, 23.VI.2017, leg. KI, KJ, PA, TB; Constanţa County: Esechioi (forest), 7.VII.2014, leg. KI, OA, PA, SzL.

\section{Protapion ononidis (GYLLENHAL, 1827)}

New data: Tulcea County: Frecăţei, Celic Dere Monastery (hillside) 31.V.2005, leg. CsS; Babadag (forest), 13.IX.2016, leg. KI, KJ, PA, TB.

\section{Protapion schoenherri (BOHEMAN, 1839)}

New data: Tulcea County: Slava Rusă, 1-3.V.2014, leg. KI, IZ, PA, SzL; Nifon, pârâul Pârlita valley, 23.VI.2017, leg. KI, KJ, PA, TB; Smârdan, Braţul Măcin, 22.VI.2017, leg. KI, KJ, PA, TB; N from Nifon, 23.VI.2017, leg. KI, KJ, PA, TB; $2,5 \mathrm{~km}$ from Stejaru to NW, 25.VI.2017, leg. KI, KJ, PA, TB; 1,5 $\mathrm{km}$ from Beidaud to SW, 25.VI.2017, leg. KI; $2 \mathrm{~km}$ from Slava Rusă to NE, 3.VI.2018, leg. FK, KI, KJ, PA; Horea, 3.VI.2018, leg. KI; Constanţa County: Cotu Văii, 23.VII.1997, leg. CP(Coll. Mus. G. ANTIPA) - Esechioi (forest), 7.VII.2014, leg. KI, OA, PA, SzL; Oltina, 8.VII.2014, leg. KI, OA, PA, SzL; Băneasa, Canaraua Fetii, (forest), 17.IX.2016, leg. KI, KJ, PA, TB; Cobadin, 14.V.2017, leg. FK, KI, KJ, PA; Albeşti, Pădurea Hagieni (forest), 12.V.2017, leg. KI.

\section{Protapion trifolii (LINNÉ, 1768)}

Published data: FLECK 1905

New data: Constanţa County: Cotu Văii, 23.VII.1997, leg. CP (Coll. Mus. G. ANTIPA) - Balta Limanu, 5-6. VII.2014, leg. KI, OA, PA, SzL; Esechioi (forest), 7.VII.2014, leg. KI, OA, PA, SzL; Pădurea Hagieni (forest), 6.VII.2014, leg. KI, OA, PA, SzL, Albeşti, Pădurea Hagieni (forest), 12.V.2017, leg. FK, KI, KJ, PA; Limanu, 12.V.2017, leg. FK, KI, KJ, PA; Hagieni, Pădurea Hagieni (forest), 13.V.2017, leg. FK, KI, KJ, PA; 23 August, 13.V.2017, leg. FK, KI, KJ, PA; Amzacea, 14.V.2017, leg. KI.

\section{Protapion varipes (GERMAR, 1817)}

Published data: RADUTA 2014
New data: Tulcea County: Măcin, leg. ALM, (Coll. Mus. G. AntipA) - Enisala (castle), 10.V.2017, leg. FK, KI, KJ, PA.

\section{Pseudoprotapion elegantulum (GERMAR, 1818)}

Published data: MonTANDON 1908

New data: Tulcea County: Măcin, Greci, leg. ALM (Coll. Mus. G. ANTIPA).

\section{NANOPHYINAE GISTEL, 1848}

Corimaliini Alonso-Zarazaga, 1989

*Allomalia quadrivirgata (COSTA, 1863) (fig. 7)

New data: Tulcea County: Măcin, 7.V.2017, leg. KI; Jijila, 19.VI.2019, leg. KI, KJ, PA, TB; Constanţa County: Neptun-Olimp, Pădurea Comorova, 13.V.2017, leg. FK, KI, KJ, PA.

*Corimalia fausti fausti (ReITTER, 1890) (fig. 8)

New data: Tulcea County: Măcin, 7.V.2017, leg. FK, KI, KJ, PA; Jijila, râul Jijila, 23.VI.2017, leg. KI, KJ, PA, TB, 19.VI.2019, leg. KI, KJ, PA, TB; Constanţa County: Vadu env., Grindul Chituc, 21.IX.2010, leg. JK, 6.VI.2018, leg. FK, KI, KJ, PA; Istria, Cetatea Histria, 26-28.VI.2017, leg. KI, 6.VI.2018, leg. KI.

\section{Corimalia tamarisci (GYLLENHAL, 1838)}

(= Corimalia languida BoHEMAN, 1845)

Published data: IEniştea 1974, Teodor and Manole 1996, Procheş 1999, Raduta 2014

\section{Hypophyes minutissimus (TOURNIER, 1868)}

Published data: Teodor and Manole 1996, Procheş 1999, RADUTA 2014

New data: Tulcea County: Smârdan, Braţul Măcin, 28.VI.2017, leg. KI, KJ, PA, TB; Jijila, râul Jijila, 23.VI.2017, leg. KI, KJ, PA, TB, 19.VI.2019, leg. KI, KJ, PA, TB; Constanţa County: Neptun-Olimp, Pădurea Comorova, 13.V.2017, leg. FK, KI, KJ, PA; Istria, Cetatea Histria, 26.VI.2017, leg. KI, 6.VI.2018, leg. KI; Vadu, Grindul Chituc, 6.VI.2018, leg. FK, KI, KJ, PA.

\section{Nanophyini Gistel, 1848}

Dieckmanniellus chevrieri (BOHEMAN, 1845)

(= Nanophyes helveticus TourNIER, 1867)

New data: Tulcea County: Smârdan, Braţul Măcin, 22.VI.2017, leg. KI, KJ, PA, TB, 2.VI.2018, leg. FK, KI, KJ, PA; Greci (village), 22.VI.2017, leg. KI, KJ, PA, TB, 8.V.2017, leg. KI; Constanţa County: Vadu env., Grindul Chituc, 21.IX.2010, leg. JK.

Dieckmanniellus gracilis (L. REDTENBACHER, 1849) New data: Tulcea County: Smârdan, Braţul Măcin, 7.V.2017, leg. FK, KI, KJ, PA.

\section{Dieckmanniellus nitidulus (GYLLENHAL, 1838)}

Published data: MonTANDON 1887

New data: Constanţa County: Esechioi (forest), 1-2. IV.2016, leg. FK, KI, PA, SzL; Istria, Cetatea Histria, 28.VI.2017, leg. KI, KJ, PA, TB; 2 km from Sinoie to SE, 27.VI.2017, leg. KI, KJ, PA, TB. 


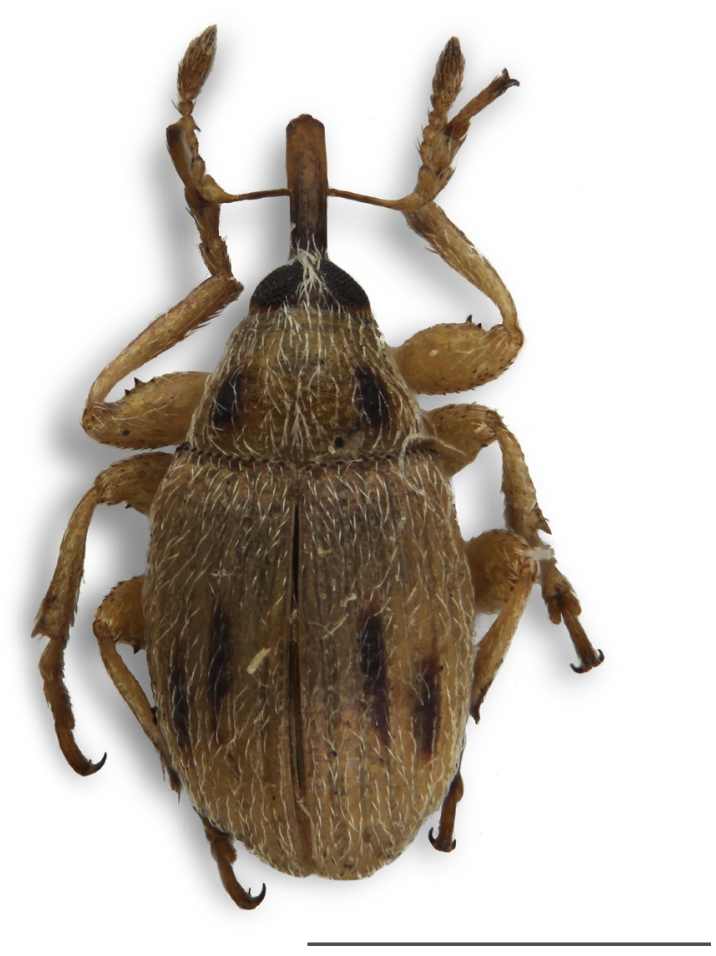

Fig. 7. Allomalia quadrivirgata (CosTA, 1863). Scale bar: 1 mm. Photo Zoltán CsaTA

Nanomimus hemisphaericus (OLIVIER,1807)

New data: Tulcea County: Smârdan, Braţul Măcin, 2.VI.2018, leg. FK, KI, KJ, PA.

Nanophyes brevis brevis BOHEMAN, 1845

New data: Tulcea County: Smârdan, Braţul Măcin, 22.VI.2017, leg. KI, KJ, PA, TB; $2,7 \mathrm{~km}$ from Horia to S, 28.VI.2017, leg. KI, KJ, PA, TB; Constanţa County: Istria, Cetatea Histria, 14.IX.2016, leg. KI.

Nanophyes marmoratus marmoratus (GoEZE, 1777) Published data: MonTANDON 1908

New data: Tulcea County: Frecăţei, Celic Dere Monastery (hillside) 31.V.2005, leg. CsS; Periprava, 1.VII.2005, leg. DDC; Enisala (wooded pasture), 23.IX.2016, leg. KI, KJ, PA, TB; Smârdan, Braţul Măcin, 22.VI.2017, leg. KI, KJ, PA, TB, 2.VI.2018, leg. FK, KI, KJ, PA; $2,7 \mathrm{~km}$ from Horia to $\mathrm{S}$, 28.VI.2017, leg. KI, KJ, PA, TB; Constanţa County: Furnica, Rez. Dumbrăveni, 13-14.V.1995, leg. CP (Coll. Mus. G. Antipa) - Istria, Cetatea Histria, 2628.VI.2017, leg. KI, KJ, PA, TB; Sinoie, Grindul Lupilor, 22.VI.2019, leg. KI, KJ, PA, TB.

CURCULIONIDAE LATREILLE, 1802

BAGOINAE C. G. Thomson, 1859

Bagous (Bagous) bagdatensis PIC, 1904 (= wagneri DieCKMANN, 1964)

Published data: Holecova 1993

New data: Tulcea County: Smârdan, Brațul Măcin, 21-23.IX.2015, leg. KI, KJ, PA, 11.IX.2016, leg. KI, KJ, PA, TB, 7.V.2017, leg. FK, KI, KJ, PA, 28.VI.2017, leg. KI, KJ, PA, TB, 2.VI.2018, leg. FK,

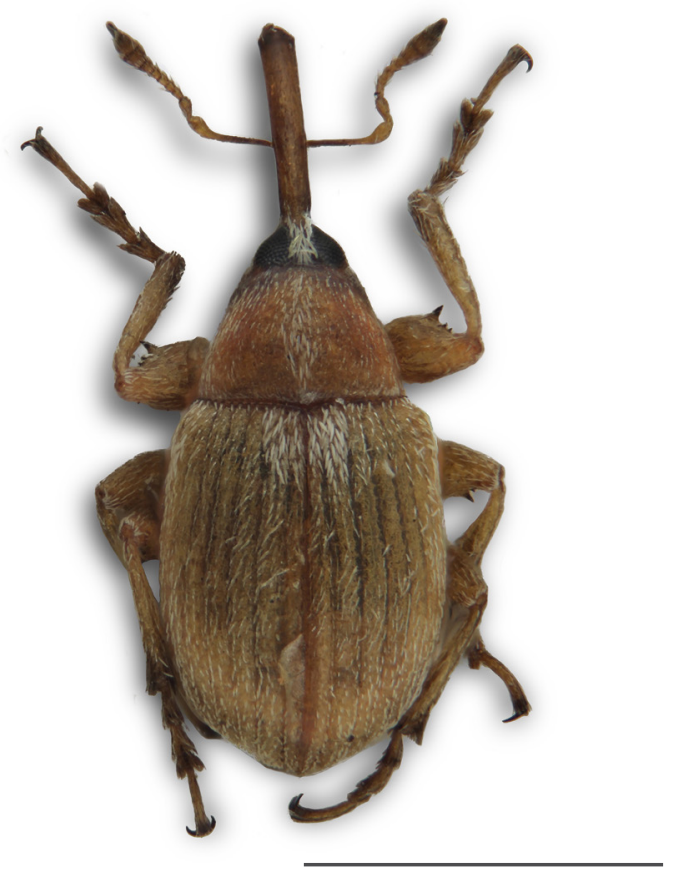

Fig. 8. Corimalia fausti fausti (ReITTER, 1890). Scale bar: 1 mm. Photo Zoltán CsaTA

KI, KJ,PA; Nifon, pârâul Pârlita valley, 23.VI.2017, leg. KI, KJ, PA, TB.

Bagous (Bagous) binodulus (HERBST, 1795)

Published data: Montandon 1906, Negru and RoşCa 1967, RADUTA 2014

New data: Tulcea County: Periprava, Păd. Letea, 23.VI.1966, leg. ŞN (Coll. Mus. G. ANTIPA) Periprava, 1.VII.2005, leg. DDC.

Bagous (Bagous) glabrirostris (HERBST, 1795)

Published data: Fleck 1905, Negru and RoşCa 1967, RADUTA 2014

New data: Tulcea County: Caraorman, 3.VI.1968, leg. ID (Coll. Mus. G. ANTIPA) - Smârdan, Braţul Măcin, 22.VI.2017, leg. KI, KJ, PA, TB, 2.VI.2018, leg. FK, KI, KJ, PA; Greci (village), 22.VI.2017, leg. KI, KJ, PA, TB.

\section{Bagous (Bagous) lutulentus (GYLLENHAL, 1813) Published data: FLECK 1905 \\ New data: Tulcea County: Smârdan, Braţul Măcin, 2.VI.2018, leg. FK, KI, KJ, PA.}

Bagous (Bagous) puncticollis BOHEMAN, 1845 New data: Tulcea County: Caraorman, 3.VI.1968, leg. ID (Coll. Mus. G. ANTIPA) - Periprava, 1.VII.2005, leg. DDC; Parcheş, 23.IX.2015, leg. KI, KJ, PA; Smârdan, Brațul Măcin, 11.IX.2016, leg. KI, 22.VI.2017, leg. KI, KJ, PA, TB, 2.VI.2018, leg. FK, KI, KJ, PA; Greci (village), 22.VI.2017, leg. KI, KJ, PA, TB; Constanţa County: Sinoie, Grindul Lupilor, 22.VI.2019, leg. KI, KJ, PA, TB. 
Bagous (Bagous) robustus H. BRISOUT de BARNEVILLE, 1863

New data: Tulcea County: Smârdan, Brațul Măcin, 22.VI.2017, leg. KI, 2.VI.2018, leg. FK, KI, KJ, PA.

Bagous (Bagous) subcarinatus GYLLENHAL, 1836

Published data: MonTANDON 1887

New data: Tulcea County: Celic Dere, 24.V.1997, leg. CP (Coll. Mus. G. ANTIPA) - Smârdan, Braţul Măcin, 21-23.IX.2015, leg. KI, KJ, PA, 11.IX.2016, leg. KI, KJ, PA, TB, 7.V.2017, leg. KI, 22.VI.2017, leg. KI, KJ, PA, TB, 2.VI.2018, leg. FK, KI, KJ, PA; Slava Rusă, 23.IX.2015, leg. KI, KJ, PA; Codru Monastery, 24.VI.2017, leg. KI, KJ, PA, TB; M-ţii Măcin, Greci, 18-19.VI.2019, leg. KI, KJ, PA, TB; Sinoie, 23.VI.2019, leg. KI, KJ, PA, TB; Constanţa County: Sinoie, Grindul Lupilor, 22.VI.2019, leg. KI, KJ, PA, TB.

\section{Bagous (Macropelmus) argillaceus GYLLENHAL, 1836 \\ Published data: Montandon 1887, 1908; FleCK} 1905; Negru and RoşCa 1967; RadUTA 2014

New data: Tulcea County: Sulina, 7.VII.1968, leg. ŞN (Coll. Mus. G. ANTIPA) - Braţul Sfântu Gheorghe, 2122.VI.2003, leg. TB; M-ţii Măcin (hillside, 200 m), 29.V.2005, leg. CsS; $1 \mathrm{~km}$ NW of Murighiol, Lacul Porculeţ (lake), 7-8.VI.2008, leg. PJ; Smârdan, Braţul Măcin, 22.VI.2017, leg. KI, KJ, PA, TB, 2.VI.2018, leg. FK, KI, KJ, PA; Constanţa County: Păd. Hagieni, 19.VI.1962, leg. AtR (Coll. Mus. G. Antipa) - Balta Limanu, 5-6.VII.2014, leg. KI, OA, PA, SzL; Gura Dobrogei, 25.VI.2017, leg. KI, KJ, PA, TB; Istria, Cetatea Histria, 26-28.VI.2017, leg. KI, 21.VI.2019, leg. KI, KJ, PA, TB; Sinoie, 21, 23.VI.2019, leg. KI, KJ, PA, TB.

*Bagous (Macropelmus) claudicans BOHEMAN, 1845 New data: Tulcea County: Smârdan, Braţul Măcin, 2.VI.2018, leg. FK, KI, KJ, PA.

Bagous (Macropelmus) collignensis (HERBST, 1797)

Published data: MonTANDON 1887

*Bagous (Macropelmus) dieckmanni GRATSHEV, 1993 (fig. 9)

New data: Tulcea County: Smârdan, Braţul Măcin, 2.VI.2018, leg. FK, KI, KJ, PA.

Bagous (Macropelmus) elegans (FABRICIUS, 1801)

Published data: IENIŞTEA 1968, KoDADA and Holecova 1994

Bagous (Macropelmus) frit (HERBST, 1795)

Published data: Montandon 1887, Fleck 1905, Negru and RoşCa 1967, Raduta 2014

Bagous (Macropelmus) limosus (GYLLENHAL, 1827) New data: Tulcea County: Smârdan, Braţul Măcin, 22.VI.2017, leg. TB.

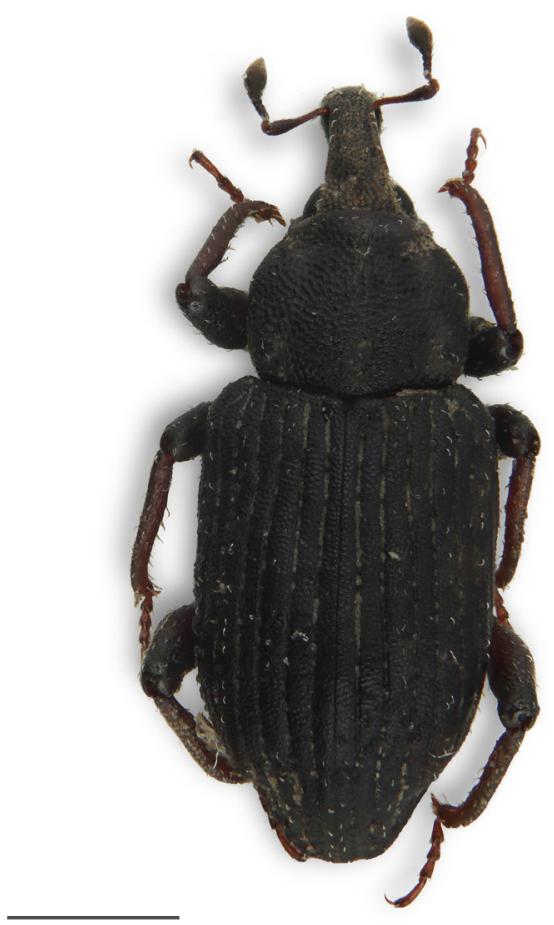

Fig. 9. Bagous (Macropelmus) dieckmanni GRATSHEV, 1993. Scale bar: $1 \mathrm{~mm}$. Photo Zoltán CsaTA

Bagous (Macropelmus) longitarsis C. G. THOMSON, 1868

New data: Tulcea County: Smârdan, Braţul Măcin, 2.VI.2018, leg. KI.

*Bagous (Macropelmus) lothari CALDARA \& O'BRIEN, 1998

New data: Tulcea County: $1.5 \mathrm{~km} \mathrm{~N}$ of Horia, Lacul Horia (lake), 8.VI.2008, leg. PJ.

Bagous (Macropelmus) lutosus (GYLLENHAL, 1813)

Published data: Montandon 1887, Fleck 1905, Negru and Roşca 1967, Teodor and Manole 1996, RADUTA 2014

New data: Tulcea County: Smârdan, Braţul Măcin, 22.VI.2017 leg. KI, KJ, PA, TB.

Bagous (Macropelmus) majzlani KODADA, HOLECOVÁ and BeHNe, 1992

Published data: Kodada and Holecova 1994

Bagous (Macropelmus) nodulosus GYLLENHAL, 1836 Published data: Fleck 1905, Teodor 1993, TeOdor and MANOLE 1996, Raduta 2014

New data: Tulcea County: $1 \mathrm{~km} \mathrm{NW}$ of Murighiol, Lacul Porculeţ (lake), 7-8.VI.2008, leg. PJ; Smârdan, Braţul Măcin, 11.IX.2016, leg. KI, 22.VI.2017, leg. KI, KJ, PA, TB, 2.VI.2018, leg. FK, KI, KJ, PA.

Bagous (Macropelmus) rotundicollis BOHEMAN, 1845 New data: Tulcea County: Smârdan, Braţul Măcin, 21-23.IX.2015, leg. KI, KJ, PA. 
Bagous (Macropelmus) rufimanus PÉRICART, 1989 New data: Tulcea County: $1 \mathrm{~km}$ NW of Murighiol, Lacul Porculeţ (lake), 7-8.VI.2008, leg. PJ.

Bagous (Macropelmus) tempestivus (HERBST, 1795) Published data: MonTANDON 1908

Bagous (Macropelmus) validus ROSENHAUER, 1847 New data: Tulcea County: Smârdan, Braţul Măcin, 7.V.2017, leg. FK, KI, KJ, PA, 22.VI.2017, leg. KI, KJ, PA, TB, 2.VI.2018, leg. FK, KI, KJ, PA.

Hydronomus alismatis MARSHAM, 1802

New data: Tulcea County: Pădurea Letea (forest), 17.VI.1993, leg. TLA; Smârdan, Braţul Măcin, 2.VI.2018, leg. FK, KI, KJ. PA.

BRACHYCERINAE BILLBERG, 1820

Brachycerini BILLBERG, 1820

Brachycerina BILLBERG, 1820

Brachycerus foveicollis GYLLENHAL, 1833

Published data: JAQUET 1898c, FLECK 1905, Montandon 1908; Negru and RoşCa 1967; Raduta 2014

New data: Constanţa County: Murfatlar, Fântâniţa, 22.V.2015, leg. PA.

\section{*Brachycerus lutosus GYLLENHAL, 1833}

New data: Tulcea County: Babadag, 23.V.2011, leg. SzK; Constanţa County: Băneasa, Canaraua Fetii, 10.VI.1960, leg. NS; Esechioi, 18.VI.1960, leg. NS, 25.IV.1963, leg. NS, 27.IV.1963, leg. NS, 10.V.1963, leg. NS, 11.V.1963, leg. NS; Babadag, Codru, 1.VI.1970, leg. NS (Coll. Mus. G. Antipa).

Brachycerus sinuatus Olivier, 1807

Published data: TeOdor 1993, Raduta 2014

Erirhinini SCHÖNHERR, 1825

Erirhinina SCHÖNHERR, 1825

Bagoopsis globicollis (FAIRMAIRE, 1863) (= Erirrhinus globicollis FAIRMAIRE, 1863; = Echinocnemus confusus FAUST, 1887)

New data: Tulcea County: Smârdan, Braţul Măcin, 2.VI.2018, leg. FK, KI, KJ, PA.

\section{Icaris sparganii (GYLLENHAL, 1835)}

Published data: Teodor and Manole 1996, Raduta 2014

New data: Tulcea County: Jijila, 19.VI.2019, leg. KI, KJ, PA, TB; Constanţa County: Istria env., Cetatea Histria, 2-4.V.2009, leg. JK, KP.

Lepidonotaris petax (C. R. SAHLBERG, 1823)

Published data: Procheş 1999

Notaris acridulus (LINNÉ, 1758)

Published data: Fleck 1905, Montandon 1908
Notaris scirpi (FABRICIUS, 1792)

Published data: Montandon 1887, Fleck 1905, Negru and Roşca 1967, Raduta 2014

New data: Tulcea County: Sulina, 24.VII.1964, leg. ŞN, 8-9.VII.1969, leg. APG; Periprava, 28.VII.1964, leg. ŞN; Caraorman, Delta Dunării, 21-22.VII.1979, leg. APG; Maliuc, Mila 26, 7.VIII.1991, leg. IM (Coll. Mus. G. ANTIPA) - M-ţii Măcin (hillside, 200 m), 29.V.2005, leg. CsS; Parcheş, 23.IX.2015, leg. KI, KJ, PA; Smârdan, Braţul Măcin, 7.V.2017, leg. KI; Constanţa County: Hagieni, 30.VI.1973, leg. APG (Coll. Mus. G. AntiPA) - Balta Limanu, 5-6. VII.2014, leg. KI, OA, PA, SzL.

\section{Thryogenes festucae (HERBST, 1795)}

New data: Tulcea County: Parcheş, 23.IX.2015, leg. KI; Smârdan, Braţul Măcin, 22.VI.2017, leg. KI, KJ, PA, TB, 2.VI.2018, leg. FK, KI, KJ, PA; Jijila, 19.VI.2019, leg. KI, KJ, PA, TB.

Tournotaris bimaculata (FABRICIUS, 1787)

Published data: TeOdor and Manole 1996, Raduta 2014

Tanysphyrini GisTel, 1848

*Stenopelmus rufinasus GYLlENHAL, 1835 (fig. 10) New data: Tulcea County: Maliuc, Mila 26, 8.VIII.1991, leg. IM (Coll. Mus. G. ANTIPA) Smârdan, Braţul Măcin, 11.IX.2016, leg. PA; M-ţii Măcin, Greci, 8.V.2017, leg. KI.

*Tanysphyrus ater BLATCHLEY, 1928 (= makolskii SMRECZYŃSKI, 1957)

New data: Tulcea County: Enisala, 10.V.2017, leg. FK.

\section{Tanysphyrus lemnae (PAYKULL, 1792)}

Published data: Montandon 1887, Fleck 1905, Negru and Roşca 1967, Teodor and Manole 1996, RADUTA 2014

New data: Tulcea County: Smârdan, Braţul Măcin, 21-23.IX.2015, leg. KI, KJ, PA, 11.IX.2016, leg. KI, KJ, PA, TB, 7.V.2017, leg. FK, KI, KJ, PA, 22.VI.2017, leg. KI, KJ, PA, TB, 2.VI.2018, leg. FK, KI, KJ, PA; 2,7 km from Horia to S, 28.VI.2017, leg. KI, KJ, PA, TB; Constanţa County: Sinoie, Grindul Lupilor, 27.VI.2017, leg. KI, KJ, PA, TB.

CONODERINAE SCHÖNHERR, 1833

BARIDITAE SCHÖNHERR, 1836

Apostasimerini SCHÖNHERR, 1844

Zygobaridina PIERCE, 1907

Limnobaris t-album (LINNÉ, 1758)

Published data: IENIŞTEA 1974

New data: Tulcea County: M-ţii Măcin (hillside, 200 m), 29.V.2005, leg. CsS; Caraorman, 14-15.V.1992, leg. TLA; Constanţa County: Istria env., Cetatea Histria, 2-4.V.2009, leg. JK. 


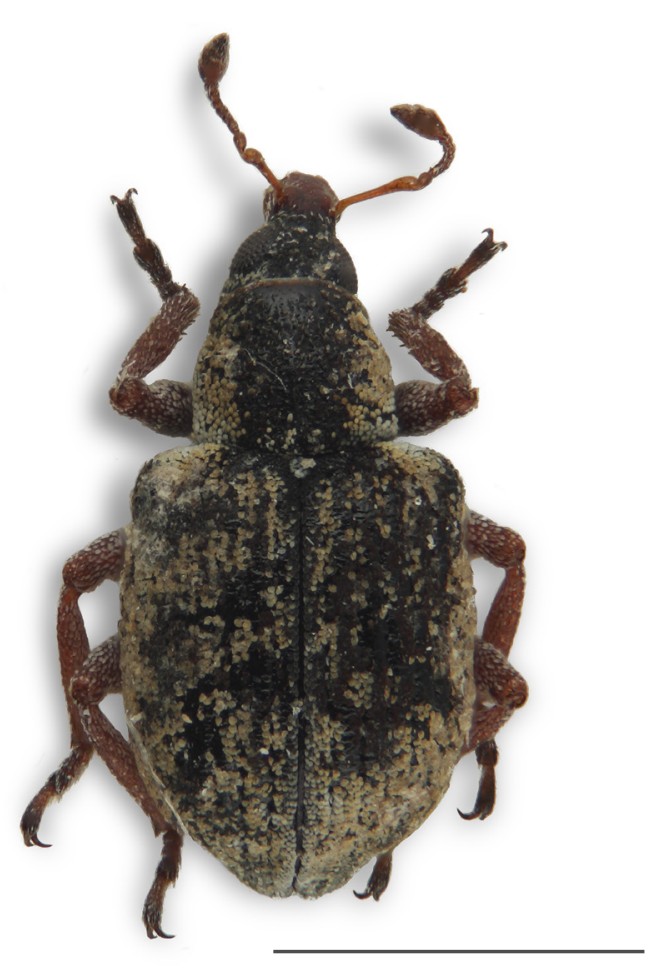

Fig. 10. Stenopelmus rufinasus Gyllenhal, 1835. Scale bar: $1 \mathrm{~mm}$. Photo Zoltán CsATA

Baridini SCHÖNHERR, 1836

Baridina SCHÖNHERR, 1836

[Aulacobaris andalusiaca (H. BRISOUT de

BARNEVILLE, 1870)]

Published data: JAQUET 1899a - Living in Spain only. Incorrect identification.

Aulacobaris angusta angusta (BRULLÉ, 1832)

Published data: FleCK 1905, Montandon 1908

New data: Tulcea County: Măcin, leg. ALM (Coll. Mus. G. ANTIPA) - Enisala, 24-25.V.2013, leg. KI, PA, RI, SzL.

\section{Aulacobaris coerulescens coerulescens (SCOPOLI, 1763)}

Published data: Montandon 1908, Negru 1957, Negru and RoşCa 1967, Raduta 2014

New data: Tulcea County: Celic Dere, 20.V.1997, leg. CH; Izvoarele, 21.V.1997, leg. RS (Coll. Mus. G. ANTIPA) - Enisala, 24-25.V.2013, leg. KI, PA, RI, SzL, 20.VI.2019, leg. KI; Codru Monastery, 30-31. III.2016, leg. FK, KI, PA. SzL; Constanţa County: Gura Dobrogei, 28-29.IV.2012, leg. KI, 26.V.2013, leg. KI, PA, RI, SzL, 30.IV-2.V.2014, leg. KI, IZ, PA, SzL, 12.V.2017, leg. FK, KI, KJ, PA, 25-27.VI.2017, leg. KI, KJ, PA, TB, 7.VI.2018, leg. FK, KI, KJ, PA; Băneasa, Canaraua Fetii, 23.V.2015, leg. PA, RI, SzK, SzL, 2-3.IV.2016, leg. FK, KI, PA. SzL; Hagieni, Pădurea Hagieni (forest), 13.V.2017, leg. FK, KI, KJ, PA; $2 \mathrm{~km}$ from Sinoie to SE, 27.VI.2017, leg. KI, KJ, PA, TB; Murfatlar, Fântâniţa, 7-8.VI.2018, leg. KI.

Aulacobaris gudenusi (SCHULTZE, 1901)
New data: Tulcea County: Măcin, Valea Suluc, 27.V.2005, leg. IM (Coll. Mus. G. ANTIPA) - Constanța County: Păd. Hagieni, 6.VI.1965, leg. ŞN (Coll. Mus. G. ANTIPA) - Cochirleni, 24.V.2015, leg. PA, RI, SzK, SzL; Gura Dobrogei, 12.V.2017, leg. FK, KI, KJ, PA.

Aulacobaris janthina (BOHEMAN, 1836)

New data: Tulcea County: M-ţii Măcin, to the Peak Ţuţuiatu, 28.IV.2010, leg. TLA; Constanţa County: Pădurea Letea (forest), 17.VI.1993, leg. det. TLA; Băneasa, Canaraua Fetii, 23.V.2015, leg. PA, RI, SzK, SzL.

Aulacobaris cf. janthina (BoHEMAN, 1836)

New data: Tulcea County: S of Horia, 6.V.2009, leg. $\mathrm{JK}$.

\section{*Aulacobaris kiesenwetteri (FAUST, 1890)}

New data: Tulcea County: Codru env., 9-10.VI.2005, leg. SL.

\section{Aulacobaris lepidii (GERMAR, 1823)}

Published data: HurmuZachi 1904, Montandon 1908 New data: Tulcea County: M-ţii Măcin, Valea Fagilor, 30.V.2005, leg. CsS; Slava Rusă, 24-25.V.2013, leg. KI, PA, RI, SzL; Enisala (castle), 21.V.2015, leg. PA, RI, SzK, SzL; Smârdan, Braţul Măcin, 28.VI.2017, leg. KI, KJ, PA, TB; M-ţii Măcin, Nifon (forest), 23.VI.2017, leg. KI, KJ, PA, TB; $3 \mathrm{~km}$ from Slava Rusă to NE, 24.VI.2017, leg. KI; Constanţa County: Gura Dobrogei, 1.IV.2016, leg. FK, KI, PA. SzL.

Aulacobaris picicornis picicornis (MARSHAM, 1802) Published data: MonTANDON 1908

New data: Tulcea County: Măcin, leg. ALM (Coll. Mus. G. ANTIPA) - Enisala, 24-25.V.2013, leg. KI, PA, RI, SzL; Codru Monastery, 19.V.2015, leg. PA, RI，SzK，SzL，20.VI.2019， leg. KI，KJ，PA，TB; M-ții Măcin, Greci, 7.V.2017, leg. FK, KI, KJ, PA; Constanţa County: Hârşova, leg. ALM; Agigea, 27.V.1963, leg. XS; Furnica, Rez. Dumbrăveni, 1314.V.1995, leg. CP (Coll. Mus. G. AnTIPA) - Palazu Mic, 22.V.2015, leg. PA, RI, SzK, SzL.

[Aulacobaris prasina (BOHEMAN, 1836)]

Published data: JAQUET 1899a, FLECK 1905 - Spread: Western Europe. Incorrect identification.

Aulacobaris violaceomicans violaceomicans (A SOLARI \& F. SOlARI, 1905)

Published data: MonTANDON 1906, 1908

Baris analis analis (OLIVIER, 1791)

Published data: TeOdor and Manole 1996, Raduta 2014

Baris artemisiae (PANZER, 1794)

Published data: Montandon 1887, 1908; FleCK 1905 New data: Tulcea County: Cerna, Parcul Naţional 


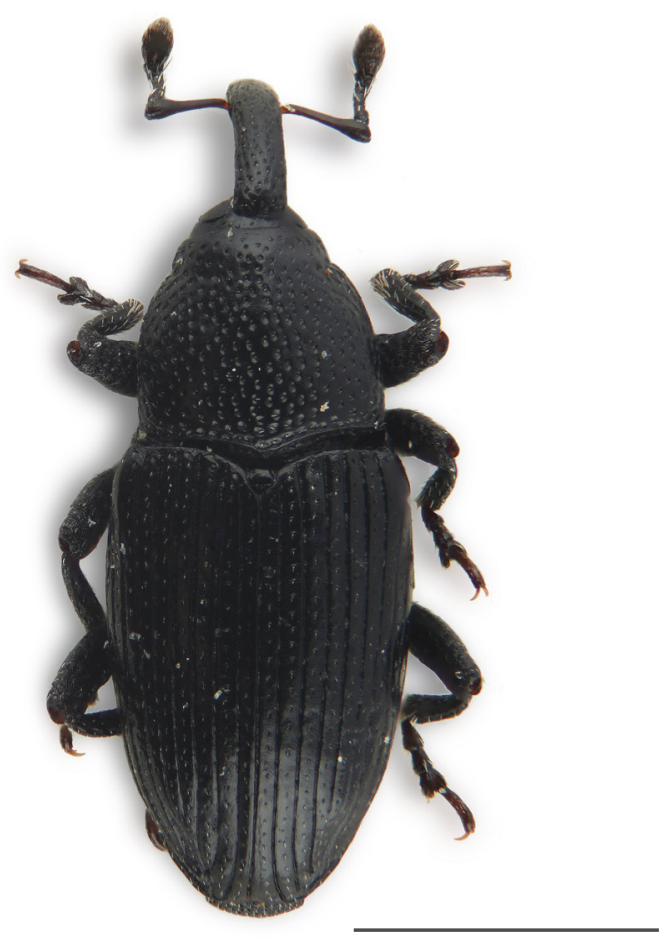

Fig. 11. Labiaticola despicatus FAUST, 1889. Scale bar: $1 \mathrm{~mm}$. Photo Zoltán CsATA

M-ții Măcin, 26.V.2005, leg. IM (Coll. Mus. G. ANTIPA) - M-ții Măcin, Greci, 7.V.2017, leg. FK, KI, KJ, PA; Slava Rusă, 9.V.2017, leg. FK, KI, KJ, PA; Nifon, pârâul Pârlita valley, 23.VI.2017, leg. KI, KJ, PA, TB; Constanţa County: Băneasa, Canaraua Fetii, 20.V.1993, leg. CH (Coll. Mus. G. ANTIPA) - Ostrov, 2.IV.2016, leg. FK, KI, PA. SzL.

Baris rufescens A. SOLARI \& F. SOLARI, 1904 Published data: MonTANDON 1906

Baris sulcata (BOHEMAN, 1836)

Published data: FleCK 1905 MonTANDON 1908

\section{Cosmobaris scolopacea (GERMAR, 1819)}

Published data: Fleck 1905, Teodor and Manole 1996, RADUTA 2014

New data: Tulcea County: S from Hamcearca, 24.VI.2017, leg. KI, KJ, PA, TB; Nifon, pârâul Pârlita valley, 23.VI.2017, leg. TB; Enisala (wooded pasture), 4.VI.2018, leg. FK, KI, KJ, PA; Constanţa County: Istria, Cetatea Histria, 26-28.VI.2017, leg. KI, KJ, PA, TB, 6.VI.2018, leg. FK, KI, KJ, PA, 21.VI.2019, leg. KI, KJ, PA, TB; $2 \mathrm{~km}$ from Sinoie to SE, 5.VI.2018, leg. FK, KI, KJ, PA.

Labiaticola atricolor (BOHEMAN, 1844)

Published data: TeOdor and MANOLE 1996

New data: Tulcea County: Canalul Magearu, 17.VI.1993, leg. TLA; Codru Monastery, 2325.V.2013, leg. KI, PA, RI, SzL; Enisala, 2425.V.2013, leg. KI, PA, RI, SzL; Enisala, Cetatea Heraclea, 25.VI.2017, leg. KI; Constanţa County: 3

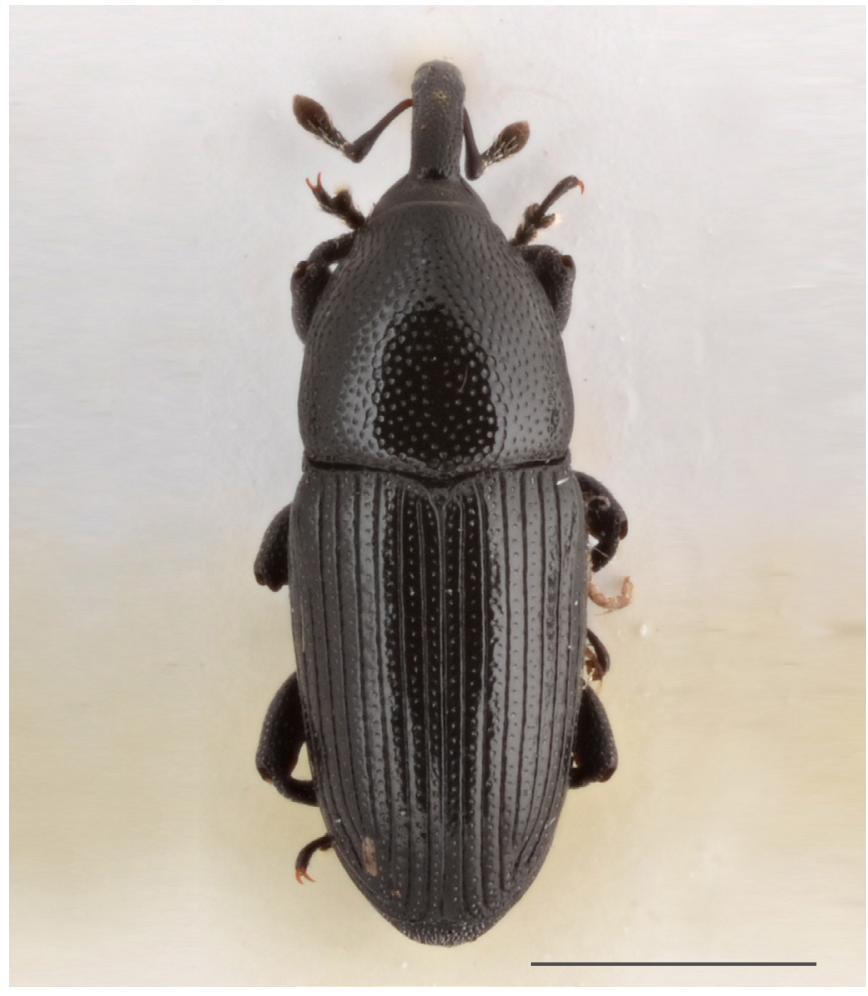

Fig. 12. Labiaticola melas BoHeman, 1836. Scale bar: $1 \mathrm{~mm}$. Photo Tamás NÉMETH

km W of Crucea, Allah Bair (Nature Reserve), 3-4. VI.2010, leg. PJ; Esechioi (forest), 7.VII.2014, leg. KI, OA, PA, SzL; Murfatlar, Fântâniţa, 22.V.2015. leg. PA, RI, SzK, SzL.

*Labiaticola despicatus FAUST, 1889 (fig. 11) New data: Tulcea County: Codru Monastery, 2325.V.2013, leg. KI, PA, RI, SzL, 25.VI.2017, leg. KI, KJ, PA, TB; Turcoaia, $6 \mathrm{~km}$ from Cerna to NW, 3.VI.2018, leg. KI; Enisala (castle), 20.VI.2019, leg. KI, KJ, PA, TB; Constanţa County: Hârşova, leg. ALM (Coll. Mus. G. AntiPA) - Gura Dobrogei, 25.VI.2017, leg. KI, KJ, PA, TB; Sinoie, Grindul Lupilor, 5.VI.2018, leg. FK, KI, KJ, PA.

*Labiaticola melas (BoHEMAN, 1836) (fig. 12) New data: Constanţa County: Murfatlar, Fântâniţa, 7-8.VI.2018, leg. FK, KI, KJ, PA, det. B. Korotyaev.

\section{Malvaevora timida timida (RossI, 1792)}

Published data: Montandon 1908, TeOdor and MANOLE 1996, RADUTA 2014

New data: Tulcea County: C. A. Rosetti, 07. 1950., coll. IC; Maliuc, Mila 26., 8.VIII.1991, leg. IM, RS; Mănăstirea Cocoş, 24.V.2005, leg. RS (Coll. Mus. G. ANTIPA) - Insula Popina, 9.VI.1992, leg. TLA; Periprava, 1.VII.2005, leg. DDC; Codru Monastery, 30.IV-3.V.2014, leg. KI, IZ, PA, SzL; Enisala, 10.V.2017, leg. FK, KI, KJ, PA; Constanţa County: Oltina, 8.VII.2014, leg. KI, OA, PA, SzL; Palazu Mic, 22.V.2015. leg. PA, RI, SzK, SzL; Murfatlar, Fântâniţa, 22.V.2015. leg. PA, RI, SzK, SzL; 23 August, 13.V.2017, leg. FK, KI, KJ, PA. 
Melanobaris atramentaria (BOHEMAN, 1836)

Published data: Montandon 1908, Negru and RoşCa 1967, RaduTA 2014

New data: Tulcea County: Măcin, leg. ALM; C. A. Rosetti, Deltă, 10.V.1964, leg. MW; Măcin, Valea Suluc, 27.V.2005, leg. IM (Coll. Mus. G. ANTIPA) Slava Rusă, 1-3.V.2014, leg. KI, IZ, PA, SzL; Enisala (castle), 21.V.2015, leg. PA, RI, SzK, SzL, 10.V.2017, leg. FK, KI, KJ, PA; M-ţii Măcin, 5 km S Greci, 8.V.2017, leg. FK, KI, KJ, PA.

\section{Melanobaris laticollis (MARSHAM 1802)}

New data: Tulcea County: Măcin, Valea Suluc, 27.V.2005, leg. IM (Coll. Mus. G. AntiPA).

Melanobaris morio (BOHEMAN, 1844)

Published data: MonTANDON 1908

Melanobaris semistriata BOHEMAN, 1836 (= Baris striatopunctatus REITTER, 1895)

New data: Constanţa County: Pecineaga (forest), 14.V.2017, leg. FK, KI, KJ, PA.

Ulobaris loricata (BOHEMAN, 1836)

Published data: NEGRU 1968

New data: Tulcea County: Periprava, 1.VII.1965, leg. ŞN (Coll. Mus. G. ANTIPA).

\section{CEUTORHYNCHITAE GISTEL, 1848 \\ Ceutorhynchini GisTEL, 1848}

Amalorrhynchus melanarius (STEPHENS, 1831)

Published data: JAQUET 1902b, FLECK 1905

New data: Tulcea County: Slava Rusă, 1-3.V.2014, leg. KI, IZ, PA, SzL; M-ţii Măcin, Valea Fagilor (quarry), 30.V.2005, leg. CsS; Smârdan, Braţul Măcin, 22.VI.2017, leg. KI, KJ, PA, TB, 2.VI.2018, leg. FK, KI, KJ, PA.

Amalus scortillum (HeRBST, 1795) (= haemorrhous HERBST, 1795)

New data: Tulcea County: Smârdan, Braţul Măcin, 28.VI.2017, leg. KI; Constanţa County: Sinoie, Grindul Lupilor, 27.VI.2017, leg. KI.

Calosirus terminatus (HERBST, 1795)

New data: Constanţa County: Palazu Mic, 22.V.2015. leg. PA, RI, SzK, SzL.

Ceutorhynchus aeneicollis GERMAR, 1823

Published data: TeOdor and Manole 1996, Raduta 2014

New data: Tulcea County: Codru env., 2009.V.4-5, leg. JK; Enisala, 29.IV-3.V.2014, leg. KI, IZ, PA, SzL.

Ceutorhynchus alliariae H. BRISOUT de BARNEVILLE, 1860

New data: Tulcea County: Slava Rusă, 1-3.V.2014, leg. KI, IZ, PA, SzL, 20.V.2015, leg. PA, RI, SzK,
SzL; Caugagia, 1.V.2014, leg. KI, IZ, PA, SzL; Constanţa County: Furnica, Rezervaţia Dumbrăveni, 13-14.V.1995, leg. CP (Coll. Mus. G. AnTIPA).

Ceutorhynchus assimilis (PAYKULL, 1792)

(= pleurostigma MARSHAM, 1802)

Published data: JAQUet 1899b, FleCK 1905; RAdUtA 2014

New data: Tulcea County: Codru env., 3.V.2009, leg. JK; Atmagea env., 24.IX.2010, leg. JK, 28.IV.2012, leg. KI; $1.5 \mathrm{~km} \mathrm{~N}$ of Horia, Lacul Horia (lake), 24.IX.2010, leg. JK; Babadag (forest), 23-25.V.2013, leg. KI, PA, RI, SzL; Codru Monastery, 23-25.V.2013, leg. KI, PA, RI, SzL, 11.V.2017, leg. FK; Slava Rusă, 1-3.V.2014, leg. KI, IZ, PA, SzL; 2,5 km from Stejaru to NW, 25.VI.2017, leg. KI; Constanţa County: Agigea, 27.V.1963, leg. XS (Coll. Mus. G. ANTIPA) - Gura Dobrogei, 26.V.2013, leg. KI, PA, RI, SzL; Murfatlar, Fântâniţa, 22.V.2015, leg. PA, RI, SzK, SzL; Negru Vodă (Nature Reserve), 25.IX.2015, leg. KI, KJ, PA.

\section{Ceutorhynchus carinatus GYLLENHAL, 1837}

New data: Tulcea County: Codru Monastery, 2325.V.2013, leg. KI, PA, RI, SzL, 30.IV-3.V.2014, leg. KI, IZ, PA, SzL, 19.V.2015, leg. PA, RI, SzK, SzL, 10-11.V.2017, leg. FK, KI, KJ, PA; Slava Rusă, 20.V.2015, leg. PA, RI, SzK, SzL, 9.V.2017, leg. FK, KI, KJ, PA, 11.V.2017, leg. FK, KI, KJ, PA; Enisala (castle), 10.V.2017, leg. FK, KI, KJ, PA; Slava Rusă, Uspenia Monastery, 11.V.2017, leg. FK, KI, KJ, PA; M-ţii Măcin, Greci, 7.V.2017, 8.V.2017, leg. FK, KI, KJ, PA; Smârdan, Braţul Măcin, 28.VI.2017, leg. KI; Constanţa County: Cheia env., Cheia Nature Reserve, 2.V.2009, leg. JK; Gura Dobrogei, 26.V.2013, leg. KI, PA, RI, SzL, 30.IV-2.V.2014, leg. KI, IZ, PA, SzL, 12.V.2017, leg. FK, KI, KJ, PA; Băneasa, Canaraua Fetii, 23.V.2015, leg. PA, RI, SzK, SzL; 5 km SW Gura Dobrogei, above Peştera "La Adam" SW end of gorge, 28.VI.2015, leg. MGy.

\section{Ceutorhynchus chalybaeus GERMAR, 1823}

Published data: Montandon 1887, 1908; FleCK 1905 New data: Tulcea County: Celic Dere, 24.V.1997, leg. CH (Coll. Mus. G. ANTIPA) - M-ţii Măcin, Pricopan, 1.VI.2005, leg. CsS; Ciucurova env., 4.V.2009, leg. JK; Enisala, 29.IV-3.V.2014, leg. KI, IZ, PA, SzL; Enisala (castle), 21.V.2015, leg. PA, RI, SzK, SzL; Slava Rusă, 31.III.2016, leg. FK, KI, PA. SzL; M-ţii Măcin, Greci, 7.V.2017, leg. FK, KI, KJ, PA; Codru Monastery, 25.VI.2017, leg. KI, KJ, PA, TB; Turcoaia, $6 \mathrm{~km}$ from Cerna to NW, 24.VI.2017, leg. KI; Enisala (wooded pasture), 4.VI.2018, leg. KI; Constanţa County: $3 \mathrm{~km} \mathrm{~W}$ of Crucea, Allah Bair (Nature Reserve), 1.V.2009, leg. JK; Gura Dobrogei, 30.IV-2.V.2014, leg. KI, IZ, PA, SzL, 12.V.2017, leg. KI, 25.VI.2017, leg. KI, KJ, PA, TB; Istria, Cetatea Histria, 21.V.2015, leg. PA, RI, SzK, SzL; Palazu Mic, 22.V.2015. leg. PA, RI, SzK, SzL; Băneasa, Canaraua 
Fetii, 23.V.2015, leg. PA, RI, SzK, SzL; Cochirleni, 24.V.2015, leg. PA, RI, SzK, SzL, 3.IV.2016, leg. FK, KI, PA. SzL; Sinoie, Grindul Lupilor, 5.VI.2018, leg. FK, KI, KJ, PA.

Ceutorhynchus chlorophanus ROUGET, 1857

New data: Constanţa County: Furnica, Rezervaţia Dumbrăveni, 22.VI.1995, leg. CP (Coll. Mus. G. ANTIPA).

Ceutorhynchus coarctatus GYLLENHAL, 1837

Published data: Montandon 1908

New data: Tulcea County: Codru Monastery, 2325.V.2013, leg. KI, PA, RI, SzL, 10-11.V.2017, leg. FK, KI, KJ, PA; Enisala, 24-25.V.2013, leg. KI, PA, RI, SzL, 29.IV-3.V.2014, leg. KI, IZ, PA, SzL; Babadag (forest), 30.IV.2014, leg. KI, IZ, PA, SzL; Enisala (castle), 10.V.2017, leg. FK, KI, KJ, PA; 2 km from Slava Rusă to NE, 3.VI.2018, leg. KI; Constanţa County: Cheia env., Cheia Nature Reserve., 2.V.2009, leg. JK, 2.V.2014, leg. KI, IZ, PA, SzL; Gura Dobrogei, 28-29.IV.2012, leg. KI; 30.IV-2.V.2014, leg. KI, IZ, PA, SzL; Palazu Mic, 22.V.2015, leg. PA, RI, SzK, SzL.

\section{Ceutorhynchus constrictus (MARSHAM, 1802)}

New data: Tulcea County: Ciucurova env., 4.V.2009, leg. JK.

\section{Ceutorhynchus contractus (MARSHAM, 1802)}

New data: Tulcea County: Slava Rusă, 4.V.2009, leg. JK, 1-3.V.2014, leg. KI, IZ, PA, SzL; Enisala (castle), 10.V.2017, leg. FK, KI, KJ, PA; M-ţii Măcin, Nifon (forest), 23.VI.2017, leg. KI, KJ, PA, TB; Constanţa County: Furnica, Rezervaţia Dumbrăveni, 13-14.V.1995, leg. CP (Coll. Mus. G. ANTIPA) - Gura Dobrogei, 30.IV-2.V.2014, leg. KI, IZ, PA, SzL, 12.V.2017, leg. FK, KI, KJ, PA; Băneasa, Canaraua Fetii (quarry),, 23.V.2015, leg. PA, RI, SzK, SzL, 2-3. IV.2016, leg. FK, KI, PA. SzL; Esechioi (forest) 1-2. IV.2016, leg. FK, KI, PA. SzL; Limanu, 12.V.2017, leg. FK, KI, KJ, PA.

\section{Ceutorhynchus erysimi (FABRICIUS, 1787)}

Published data: JAQUET 1899a, 1899b, 1902a; FLECK 1905; Montandon 1908

New data: Tulcea County: Măcin, Valea Suluc, 27.V.2005, leg. IM, CB (Coll. Mus. G. Antipa) M-ţii Măcin (hillside, 200 m), 29.V.2005, leg. CsS; Frecăţei, Celic Dere Monastery (hillside) 31.V.2005, leg. CsS; M-ţii Măcin, Pricopan, 1.VI.2005, leg. CsS; Enisala, 29.IV-3.V.2014, leg. KI, IZ, PA, SzL; Enisala (castle), 21.V.2015, leg. PA, RI, SzK, SzL; 4 km SW Babadag, 3.VI.2016, leg. MGy; Smârdan, Braţul Măcin, 11.IX.2016, leg. KI, KJ, PA, TB; M-ţii Măcin, 5 km S Greci, 8.V.2017, leg. FK, KI, KJ, PA; M-ţii Măcin, Greci, 7.V.2017, leg. FK, KI, KJ, PA, 8.V.2017, leg. FK, KI, KJ, PA; Codru Monastery, 25.VI.2017, leg. KI, KJ, PA, TB; 2,5 km from Stejaru to NW, 25.VI.2017, leg. KI, KJ, PA, TB; M-ţii Măcin, Nifon (forest), 23.VI.2017, leg. KI, KJ, PA, TB; 2,7 km from Horia to S, 28.VI.2017, leg. KI; $2 \mathrm{~km}$ from Slava Rusă to NE, 3.VI.2018, leg. KI; Constanţa County: Hagieni, 6.VI.1965, leg. MW; Furnica, Rezervaţia Dumbrăveni, 13-14.V.1995, leg. CP (Coll. Mus. G. ANTIPA) - Gura Dobrogei, 26.V.2013, leg. KI, PA, RI, SzL, 12.V.2017, leg. FK, KI, KJ, PA, 7.VI.2018, leg. FK, KI, KJ, PA; Cheile Dobrogei, 2.V.2014, leg. KI, IZ, PA, SzL; Pădurea Hagieni (forest), 6.VII.2014, leg. KI, OA, PA, SzL, Albeşti, Pădurea Hagieni (forest), 12.V.2017, leg. FK, KI, KJ, PA; Băneasa, Canaraua Fetii (quarry), 23.V.2015, leg. PA, RI, SzK, SzL, 2-3.IV.2016, leg. FK, KI, PA. SzL; Băneasa, Canaraua Fetii (forest), 17.IX.2016, leg. KI, KJ, PA, TB; Ostrov, 2.IV.2016, leg. FK, KI, PA. SzL; 4,5 km SW Gura Dobrogei, 0.6 km WSW Peştera Liliecilor, p. Târguşorul, 31.V.2016, leg. MGy; Hagieni, Pădurea Hagieni (forest), 17.IX.2016, leg. FK, KI, KJ, PA; Istria, Cetatea Histria, 11.V.2017, leg. FK, KI, KJ, PA; Amzacea, 14.V.2017, leg. KI; Cobadin, 14.V.2017, leg. KI; Murfatlar, Fântâniţa, 7-8.VI.2018, leg. KI.

Ceutorhynchus granulicollis C. G. Thomson, 1865 New data: Tulcea County: Codru Monastery, 30-31. III.2016. leg. FK, KI, PA, SzL; Enisala (wooded pasture), 4.VI.2018, leg. KI; Constanţa County: Gura Dobrogei, 12.V.2017, leg. KI.

Ceutorhynchus griseus C. N. F. Brisout de Barneville, 1869

New data: Tulcea County: Atmagea env., 24.IX.2010, leg. JK; Constanţa County: 5 km SW Gura Dobrogei, above Peştera "La Adam" SW end of gorge, 28.VI.2015, leg. MGy.

Ceutorhynchus hampei C. N. F. BRISOUT de BARNEVILLE, 1869

New data: Tulcea County: M-ţii Măcin, Valea Fagilor (quarry), 30.V.2005, leg. CsS;

\section{Ceutorhynchus hirtulus GERMAR, 1823}

Published data: Montandon 1908

New data: Tulcea County: Periprava, 12.X.1966, leg. ŞN; Caraorman, 06.V.1967, leg. MW; Celic Dere, 24.V.1997, leg. CH (Coll. Mus. G. AnTIPA) M-ţii Măcin, Greci env., 6.V.2009, leg. JK, 8.V.2017, leg. FK, KI, KJ, PA; Enisala, 29.IV-3.V.2014, leg. KI, IZ, PA, SzL; Caugagia, 1.V.2014, leg. KI, IZ, PA, SzL; Slava Rusă, 1-3.V.2014, leg. KI, IZ, PA, SzL; 5km SW Gura Dobrogei, above Peştera "La Adam", SW end of gorge, 1.VI.2016, leg. MGy; Codru Monastery, 10-11.V.2017, leg. FK, KI, KJ, PA; Enisala (castle), 10.V.2017, leg. FK, KI, KJ, PA; Slava Rusă, 11.V.2017, leg. FK, KI, KJ, PA; M-ţii Măcin, 5 km S Greci, 8.V.2017, leg. FK, KI, KJ, PA; 2,5 km from Stejaru to NW, 25.VI.2017, leg. KI, KJ, PA, TB; 2,7 km from Horia to S, 28.VI.2017, leg. KI, KJ, PA, TB; Constanţa County: Furnica, Rezervaţia 
Dumbrăveni, 13-14.V.1995, leg. CP (Coll. Mus. G. ANTIPA) - Gura Dobrogei, 30.IV-2.V.2014, leg. KI, IZ, PA, SzL, Gura Dobrogei, 12.V.2017, leg. FK, KI, KJ, PA; Cheile Dobrogei, 2.V.2014, leg. KI, IZ, PA, SzL; Palazu Mic, 22.V.2015, leg. PA, RI, SzK, SzL; Cochirleni, 24.V.2015, leg. PA, RI, SzK, SzL; 5 km SW Gura Dobrogei, above Peştera "La Adam" SW end of gorge, 28.VI.2015, leg. MGy; 4,5 km SW Gura Dobrogei, 0,6 km WSW Peştera Liliecilor, p. Târguşorul, 28.VI.2015, leg. MGy; Limanu, 12.V.2017, leg. FK, KI, KJ, PA; Albeşti, Pădurea Hagieni (forest), 12.V.2017, leg. KI.

Ceutorhynchus ignitus Germar, 1823

Published data: TeOdor and Manole 1996

\section{Ceutorhynchus inaffectatus GYLLENHAL, 1837}

New data: Tulcea County: Slava Rusă, 24-25.V.2013, leg. KI, PA, RI, SzL, 1-3.V.2014, leg. KI, IZ, PA, SzL, 20.V.2015, leg. PA, RI, SzK, SzL; Codru Monastery, 30.IV-3.V.2014, leg. KI, IZ, PA, SzL, 10-11.V.2017, leg. FK, KI, KJ, PA; Slava Rusă, Uspenia Monastery, 11.V.2017, leg. FK, KI, KJ, PA; Constanţa County: Negureni, 21.VI.1996, leg. CP (Coll. Mus. G. Antipa) - Cheia env., Cheia Nature Reserve., 2.V.2009, leg. JK; Băneasa, Canaraua Fetii (quarry), 7.VII.2014, leg. KI, OA, PA, SzL; Murfatlar, Fântâniţa, 22.V.2015, leg. PA, RI, SzK, SzL; Gura Dobrogei, 12.V.2017, leg. FK, KI, KJ, PA.

\section{Ceutorhynchus interjectus SCHULTZE, 1903}

New data: Tulcea County: Periprava, 13.V.1964, leg. XS (Coll. Mus. G. AnTIPA) - Slava Rusă, Uspenia Monastery, 11.V.2017, leg. FK, KI, KJ, PA; Codru Monastery, 10-11.V.2017, leg. FK, KI, KJ, PA; M-ţii Măcin, Greci, 7.V.2017, leg. KI; Constanţa County: Negureni, 21.VI.1996, leg. CP (Coll. Mus. G. Antipa).

\section{*Ceutorhynchus levantinus Schultze, 1898}

New data: Tulcea County: Slava Rusă, 1-3.V.2014, leg. KI, IZ, PA, SzL; Constanţa County: Băneasa, Canaraua Fetii, 23.V.2015, leg. PA, RI, SzK, SzL.

*Ceutorhynchus merkli KoROTYAev, 2001 (fig. 13)

New data: Tulcea County: Atmagea, 28.IV.2012, leg. KI; Slava Rusă, 1-3.V.2014, leg. KI, IZ, PA, SzL; Codru env., 3.V.2009, leg. JK; Constanţa County: Cochirleni, 3.IV.2016, leg. FK, KI, PA, SzL.

\section{Ceutorhynchus nanus GyLLENHAL, 1837}

New data: Tulcea County: Enisala, 29.IV-3.V.2014, leg. KI, IZ, PA, SzL; M-ţii Măcin (hillside, 200 m), 29.V.2005, leg. CsS; Ciucurova env., 4.V.2009, leg. JK; Codru env., 5.V.2009, leg. JK; M-ţii Măcin, Greci, 7.V.2017, leg. FK, KI, KJ, PA, 8.V.2017, leg. FK, KI, KJ, PA, 18-19.VI.2019, leg. KI, KJ, PA, TB; Codru Monastery, 10-11.V.2017, leg. FK, KI, KJ, PA; Enisala (castle), 10.V.2017, leg. FK, KI, KJ, PA; S from Hamcearca, 24.VI.2017, leg. KI, KJ, PA, TB; 1,5

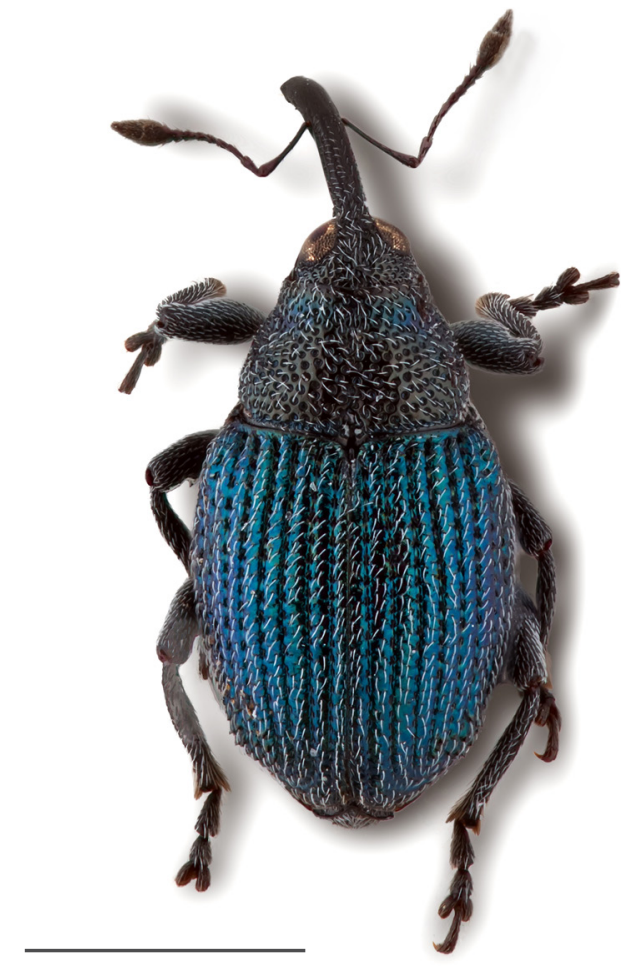

Fig. 13. Ceutorhynchus merkli Korotyaev, 2001. Scale bar: $1 \mathrm{~mm}$. Photo Zoltán CsATA

$\mathrm{km}$ from Beidaud to SW, 25.VI.2017, leg. KI, KJ, PA, TB; M-ţii Măcin, to the Peak, Ţuţuiatu, 28.IV.2010, leg. TLA; Constanţa County: Murfatlar, Fântâniţa, 4-5.VII.2014, leg. KI, OA, PA, SzL; Gura Dobrogei, 1.IV.2016, leg. FK, KI, PA, SzL, 25.VI.2017, leg. KI, KJ, PA, TB.

Ceutorhynchus nigritulus SCHULTZE, 1897

New data: Constanţa County: Gura Dobrogei, 1.IV.2016, leg. FK, KI, PA, SzL.

\section{Ceutorhynchus niyazii HoffMANn, 1957}

New data: Tulcea County: W of Horia, 6.V.2009, leg. JK; Enisala, 29.IV-3.V.2014, leg. KI, IZ, PA, SzL; Enisala (castle), 21.V.2015, leg. PA, RI, SzK, SzL, 10.V.2017, leg. FK, KI, KJ, PA; Greci (village), 8.V.2017, leg. FK, KI, KJ, PA; Constanţa County: Furnica, Rezervaţia Dumbrăveni, 13-14.V.1995, leg. CP (Coll. Mus. G. AnTIPA) - 3 km W of Crucea, Allah Bair (Nature Reserve), 1.V.2009, leg. JK; Palazu Mic, 22.V.2015, leg. PA, RI, SzK, SzL; Băneasa, Canaraua Fetii, 23.V.2015, leg. PA, RI, SzK, SzL; Cochirleni, 24.V.2015, leg. PA, RI, SzK, SzL; Costineşti (coast), 17.IX.2016, leg. KI.

\section{Ceutorhynchus obstrictus (MARSHAM, 1802)}

Published data: Montandon 1887, JAQUeT 1900b, Negru and Roşca 1967

New data: Tulcea County: Luncaviţa, Valea Fagilor, 24.V.2005, leg. IM; Măcin, Valea Suluc, 27.V.2005, leg. IM (Coll. Mus. G. ANTIPA) - M-ţii Măcin, to the Peak Ţuţuiatu, 28.IV.2010, leg. TLA; Atmagea, 28.IV.2012, leg. KI; Enisala, 24-25.V.2013, leg. KI, 
PA, RI, SzL, 29.IV-3.V.2014, leg. KI, IZ, PA, SzL; Slava Rusă, 23.IV.2009, leg. TLA, 24-25.V.2013, leg. KI, PA, RI, SzL, 1-3.V.2014, leg. KI, IZ, PA, SzL, 31.III.2016, leg. FK, KI, PA, 9.V.2017, leg. FK, KI, KJ, PA, 11.V.2017, leg. FK, KI, KJ, PA; Codru Monastery, 30.IV-3.V.2014, leg. KI, IZ, PA, SzL, 30-31.III.2016, leg. FK, KI, PA, SzL, 10-11.V.2017, leg. FK, KI, KJ, PA, 25.VI.2017, leg. KI, KJ, PA, TB, 20.VI.2019, leg. KI; 5 km SW Gura Dobrogei, above Peştera "La Adam", SW end of gorge, 1.VI.2016, leg. MGy; Slava Rusă, Uspenia Monastery, 11.V.2017, leg. FK, KI, KJ, PA; Greci (village), 8.V.2017, leg. FK, KI, KJ, PA; Enisala (castle), 10.V.2017, leg. FK, KI, KJ, PA, 20.VI.2019, leg. KI, KJ, PA, TB; M-ţii Măcin, Greci, 7.V.2017, leg. FK, KI, KJ, PA, 18-19. VI.2019, leg. KI, KJ, PA, TB; Smârdan, Braţul Măcin, 28.VI.2017, leg. KI, KJ, PA, TB, 18.VI.2019, leg. KI; $\mathrm{N}$ from Nifon, 23.VI.2017, leg. KI, KJ, PA, TB; 1,5 km from Beidaud to SW, 25.VI.2017, leg. KI, KJ, PA, TB; 2,5 km from Stejaru to NW, 25.VI.2017, leg. KI, KJ, PA, TB; Enisala, Cetatea Heraclea, 25.VI.2017, leg. KI, KJ, PA, TB; $2,7 \mathrm{~km}$ from Horia to S, 28.VI.2017, leg. KI, KJ, PA, TB; S from Hamcearca, 24.VI.2017, leg. KI, KJ, PA, TB; Turcoaia, $6 \mathrm{~km}$ from Cerna to NW, 3.VI.2018, leg. FK, KI, KJ, PA; 2 km from Slava Rusă to NE, 3.VI.2018, leg. FK, KI, KJ, PA; Constanţa County: Gura Dobrogei, 30.IV-2.V.2014 leg. KI, IZ, PA, SzL, 1.IV.2016, leg. FK, KI, PA, SzL, 12.V.2017, leg. FK, KI, KJ, PA, 25-27.VI.2017, leg. KI, KJ, PA, TB; Murfatlar, Fântâniţa, 4-5.VII.2014 leg. KI, OA, PA, SzL; Pădurea Hagieni (forest), 6.VII.2014, leg. KI, OA, PA, SzL; Albești, Pădurea Hagieni (forest), 12.V.2017, leg. FK, KI, KJ, PA; Esechioi (forest), 7.VII.2014, leg. KI, OA, PA, SzL, 1-2.IV.2016, leg. FK, KI, PA, SzL; Băneasa, Canaraua Fetii (quarry), 7.VII.2014, leg. KI, OA, PA, SzL, 23.V.2015, leg. PA, RI, SzK, SzL, 2-3.IV.2016, leg. FK, KI, PA, SzL; Cochirleni, 8.VII.2014, leg. KI, OA, PA, SzL, 24.V.2015, leg. PA, RI, SzK, SzL, 3.IV.2016. leg. FK, KI, PA, SzL; 5 km SW Gura Dobrogei, above Peştera "La Adam" SW end of gorge, 28.VI.2015, leg. MGy; Ostrov, 2.IV.2016, leg. FK, KI, PA. SzL; Limanu, 12.V.2017, leg. FK, KI, KJ, PA; Hagieni, Pădurea Hagieni (forest), 17.IX.2016, leg. FK, KI, KJ, PA; Pecineaga (forest), 14.V.2017, leg. FK, KI, KJ, PA; 23 August, 17.IX.2016, leg. FK, KI, KJ, PA; Cobadin, 14.V.2017, leg. FK, KI, KJ, PA; Istria, Cetatea Histria, 28.VI.2017, leg. KI, KJ, PA, TB; $2 \mathrm{~km}$ from Sinoie to SE, 27.VI.2017, leg. KI, KJ, PA, TB; Sinoie, Grindul Lupilor, 27.VI.2017, leg. KI, KJ, PA, TB; NeptunOlimp, Pădurea Comorova, 13.V.2017, leg. FK, KI, KJ, PA; Vadu, Grindul Chituc, 6.VI.2018, leg. FK, KI, $\mathrm{KJ}, \mathrm{PA}$.

Ceutorhynchus pallidactylus (MARSHAM, 1802)

(= Ceutorrynchus quadridens PANZER, 1795)

New data: Tulcea County: Slava Rusă, 23.IV.2009, leg. TLA, 1-3.V.2014, leg. KI, IZ, PA, SzL, 31.III.2016, leg. FK, KI, PA, SzL, 9.V.2017, leg. FK, KI, KJ,
PA, 11.V.2017, leg. FK, KI, KJ, PA; M-ţii Măcin, to the Peak Ţuţuiatu, 28.IV.2010, leg. TLA; Atmagea, 28.IV.2012, leg. KI; Enisala, 24-25.V.2013, leg. KI, PA, RI, SzL, 29.IV-3.V.2014, leg. KI, IZ, PA, SzL, 20.VI.2019, leg. KI; 5 km SW Gura Dobrogei, above Peştera "La Adam", SW end of gorge, 1.VI.2016, leg. MGy; M-ţii Măcin, 5 km S Greci, 8.V.2017, leg. FK, KI, KJ, PA; M-ţii Măcin, Greci (village), 7.V.2017, leg. FK, KI, KJ, PA, 22.VI.2017, leg. KI, 18-19.VI.2019, leg. KI, KJ, PA, TB; S from Hamcearca, 24.VI.2017, leg. KI; Enisala (castle), 10.V.2017, leg. FK, KI, KJ, PA; Codru Monastery, 10-11.V.2017, leg. FK, KI, KJ, PA, 25.VI.2017, leg. KI, KJ, PA, TB; Slava Rusă, Uspenia Monastery, 11.V.2017, leg. FK, KI, KJ, PA; Smârdan, Braţul Măcin, 22.VI.2017, leg. KI, KJ, PA, TB, 2.VI.2018, leg. FK, KI, KJ, PA, 18.VI.2019, leg. KI; Nifon, pârâul Pârlita valley, 23.VI.2017, leg. KI, KJ, PA, TB; N from Nifon, 23.VI.2017, leg. KI, KJ, PA, TB; M-ţii Măcin, Nifon (forest), 23.VI.2017, leg. KI, KJ, PA, TB; Turcoaia, $6 \mathrm{~km}$ from Cerna to NW, 24.VI.2017, leg. KI, KJ, PA, TB; 1,5 km from Beidaud to SW, 25.VI.2017, leg. KI, KJ, PA, TB; 2,5 $\mathrm{km}$ from Stejaru to NW, 25.VI.2017, leg. KI, KJ, PA, TB; Enisala, Cetatea Heraclea, 25.VI.2017, leg. KI, KJ, PA, TB; $2,7 \mathrm{~km}$ from Horia to S, 28.VI.2017, leg. KI, KJ, PA, TB; Horea, 3.VI.2018, leg. KI; General Praporgescu, 3.VI.2018, leg. KI; 2 km from Slava Rusă to NE, 3.VI.2018, leg. FK, KI, KJ, PA; Constanţa County: Gura Dobrogei, 28-29.IV.2012, leg. KI, 30.IV-2.V.2014, leg. KI, IZ, PA, SzL, 12.V.2017, leg. FK, KI, KJ, PA, 25.VI.2017, leg. KI, KJ, PA, TB, 7.VI.2018, leg. FK, KI, KJ, PA; Băneasa, Canaraua Fetii (quarry), 7.VII.2014, leg. KI, OA, PA, SzL, 2015.V.23, leg. PA, RI, SzK, SzL, 2-3.IV.2016, leg. FK, KI, PA, SzL; Cheile Dobrogei, 2.V.2014, leg. KI, IZ, PA, SzL; Murfatlar, Fântâniţa, 4-5.VII.2014, leg. KI, OA, PA, SzL, 22.V.2015, leg. PA, RI, SzK, SzL, 7-8.VI.2018, leg. FK, KI, KJ, PA; Pădurea Hagieni (forest), 6.VII.2014, leg. KI, OA, PA, SzL; Albeşti, Pădurea Hagieni (forest), 12.V.2017, leg. FK, KI, KJ, PA; Esechioi (forest), 7.VII.2014, leg. KI, OA, PA, SzL; 5 km SW Gura Dobrogei, above Peştera "La Adam" SW end of gorge, 28.VI.2015, leg. MGy; Negru Vodă (Nature Reserve) 25.IX.2015, leg. KI, KJ, PA; Cochirleni, 3.IV.2016, leg. FK, KI, PA, SzL; Limanu, 12.V.2017, leg. FK, KI, KJ, PA; Hagieni, Pădurea Hagieni (forest), 17.IX.2016, leg. FK, KI, KJ, PA; Pecineaga (forest), 14.V.2017, leg. FK, KI, KJ, PA; Cobadin, 14.V.2017, leg. FK, KI, KJ, PA; $2 \mathrm{~km}$ from Sinoie to SE, 27.VI.2017, leg. KI, KJ, PA, TB, 5.VI.2018, leg. KI; Sinoie, Grindul Lupilor, 27.VI.2017, leg. KI, KJ, PA, TB, 5.VI.2018, leg. FK, KI, KJ, PA; Costineşti (coast), 13.V.2017, leg. KI; Istria, Cetatea Histria, 26-28,VI.2017, leg. KI.

Ceutorhynchus parvulus C. N. F. BRISOUT de BARNEVILLE, 1869

Published data: JAQUET 1899b; FLECK 1905

New data: Tulcea County: Codru Monastery, 10- 
11.V.2017, leg. FK, KI, KJ, PA; Constanţa County: Băneasa, Canaraua Fetii (quarry), 2-3.IV.2016, leg. FK, KI, PA. SzL.

Ceutorhynchus pectoralis WeISE, 1895

New data: Tulcea County: Celic Dere, 20.V.1997, leg. RS (Coll. Mus. G. ANTIPA).

Ceutorhynchus picitarsis GYLLENHAL, 1837

Published data: Montandon 1887, Fleck 1905

New data: Tulcea County: Celic Dere, 20.V.1997, leg. CH; Izvoarele, 21.V.1997, leg. RS (Coll. Mus. G. ANTIPA) - Caraorman, 7.VII.1991, leg. TLA; Slava Rusă, 23.IV.2009, leg. TLA; Enisala, 29.IV-3.V.2014, leg. KI, IZ, PA, SzL; Enisala (castle), 21.V.2015, leg. PA, RI, SzK, SzL; Constanţa County: Furnica, Rezervaţia Dumbrăveni, 13-14.V.1995, leg. CP (Coll. Mus. G. Antipa) - Palazu Mic, 22.V.2015, leg. PA, RI, SzK, SzL; Băneasa, Canaraua Fetii, 23.V.2015, leg. PA, RI, SzK, SzL; Cochirleni, 24.V.2015, leg. PA, RI, SzK, SzL.

\section{Ceutorhynchus posthumus GERMAR, 1823}

New data: Tulcea County: 5km SW Gura Dobrogei, above Peştera "La Adam", SW end of gorge, 1.VI.2016, leg. MGy; Constanţa County: Palazu Mic, 22.V.2015, leg. PA, RI, SzK, SzL; Gura Dobrogei, 1.IV.2016, leg. FK, KI, PA. SzL.

\section{Ceutorhynchus pulvinatus GYLLENHAL, 1837}

\section{Published data: JAQUET 1902b, Montandon 1908}

New data: Tulcea County: Enisala, 24-25.V.2013, leg. KI, PA, RI, SzL, 29.IV-3.V.2014, leg. KI, IZ, PA, SzL; Enisala (castle), 21.V.2015, leg. PA, RI, SzK, SzL; M-ţii Măcin, Greci,7.V.2017, leg. FK, KI, KJ, PA; Greci (village), 8.V.2017, leg. FK, KI, KJ, PA; Codru Monastery, 10-11.V.2017, leg. KI; Constanţa County: Cheile Dobrogei, 2.V.2014, leg. KI, IZ, PA, SzL; Palazu Mic, 22.V.2015, leg. PA, RI, SzK, SzL; Băneasa, Canaraua Fetii, 23.V.2015, leg. PA, RI, SzK, SzL; Cochirleni, 24.V.2015, leg. PA, RI, SzK, SzL; Istria, Cetatea Histria, 11.V.2017, leg. FK, KI, KJ, PA; Sinoie, Grindul Lupilor, 27.VI.2017, leg. KI, KJ, PA, TB.

Ceutorhynchus puncticollis BOHEMAN, 1845

New data: Tulcea County: C. A. Rosetti, Pădurea Letea, 11.V.1964, leg. XS (Coll. Mus. G. ANTIPA) - Slava Rusă, 24-25.V.2013, leg. KI, PA, RI, SzL; Enisala (castle), 21.V.2015, leg. PA, RI, SzK, SzL; $3 \mathrm{~km}$ from Slava Rusă to NE, 24.VI.2017, leg. KI; Constanţa County: Furnica, Rezervaţia Dumbrăveni, 13-14.V.1995, leg. CP (Coll. Mus. G. AntiPA) Băneasa, Canaraua Fetii, 23.V.2015, leg. PA, RI, SzK, SzL.

\section{Ceutorhynchus pyrrhorhynchus MARSHAM, 1802}

New data: Tulcea County: Delta Dunării, Pădurea Letea, 18.V.1993, leg. IM (Coll. Mus. G. ANTIPA) Istria, Cetatea Histria, 6.VI.2018, leg. KI.
Ceutorhynchus rapae GylLENHAL, 1837

New data: Tulcea County: Valea Teilor, 22. 05. 1997, leg. CH; Greci, Parcul Naţ. M-ţii Măcin, 25.V.2005, leg. CB(Coll. Mus. G. ANTIPA) - Atmagea, 28.IV.2012, leg. KI; Slava Rusă, Uspenia Monastery, 11.V.2017, leg. FK, KI, KJ, PA; Smârdan, Braţul Măcin, 22.VI.2017, leg. KI, KJ, PA, TB; Codru Monastery, 25.VI.2017, leg. KI, KJ, PA, TB, 24-25.VI.2017, leg. KI; 3 km from Slava Rusă to NE, 24.VI.2017, leg. KI, KJ, PA, TB; Constanţa County: Hagieni, 12.VII.1964, leg. IS; Furnica, Rezervaţia Dumbrăveni, 22.VI.1995, leg. CP (Coll. Mus. G. ANTIPA) - Cheile Dobrogei, 2.V.2014, leg. KI, IZ, PA, SzL; Băneasa, Canaraua Fetii (quarry) 2-3.IV.2016, leg. FK, KI, PA, SzL; Gura Dobrogei, 27.VI.2017, leg. KI, KJ, PA, TB, 7.VI.2018, leg. FK, KI, KJ, PA; Murfatlar, Fântâniţa, 7-8.VI.2018, leg. FK, KI, KJ, PA.

Ceutorhynchus rhenanus (SCHULTZE, 1895)

Published data: Teodor and Manole 1996, Raduta 2014

\section{Ceutorhynchus roberti GYLLENHAL, 1837}

New data: Tulcea County: Turcoaia, $6 \mathrm{~km}$ from Cerna to NW, 3.VI.2018, leg. FK, KI, KJ, PA.

*Ceutorhynchus scrobicollis NeRESHEIMER \& H. WAGNER, 1924 (fig. 14)

New data: Tulcea County: Ciucurova env., 4.V.2009, leg. JK.

\section{Ceutorhynchus sisymbrii (DIECKMANN, 1966)}

New data: Tulcea County: W of Horia, 6.V.2009, leg. JK.

\section{Ceutorhynchus sophiae GYLLENHAL, 1837}

Published data: Montandon 1908

New data: Tulcea County: Măcin, Valea Suluc, 27.V.2005, leg. IM (Coll. Mus. G. ANTIPA) - M-ţii Măcin,Valea Fagilor (quarry), 30.V.2005, leg. CsS; M-ţii Măcin, Pricopan, 1.VI.2005, leg. CsS; W of Horia, 6.V.2009, leg. JK; Constanţa County: Furnica, Rezervaţia Dumbrăveni, 13-14.V.1995, leg. CP (Coll. Mus. G. AnTIPA).

\section{Ceutorhynchus striatellus SCHULTZE, 1900}

New data: Tulcea County: M-ţii Măcin, Greci, 8.V.2017, leg. FK, KI, KJ, PA; Codru Monastery, 10-11.V.2017, leg. FK, KI, KJ, PA; Jijila, râul Jijila, 23.VI.2017, leg. KI, KJ, PA, TB; Constanţa County: $3 \mathrm{~km} \mathrm{~W}$ of Crucea, Allah Bair (Nature Reserve), 1.V.2009, leg. JK, 3-4.VI.2010, PJ; Gura Dobrogei, 25.VI.2017, leg. KI, KJ, PA, TB.

Ceutorhynchus subpilosus C. N. F. BRISOUT de BARNEVILLE, 1869

New data: Tulcea County: Enisala (wooded pasture), 4.VI.2018, leg. KI; Constanţa County: $3 \mathrm{~km} \mathrm{~W}$ of Crucea, Allah Bair (Nature Reserve), 6-7.VI.2005, leg. LS. 


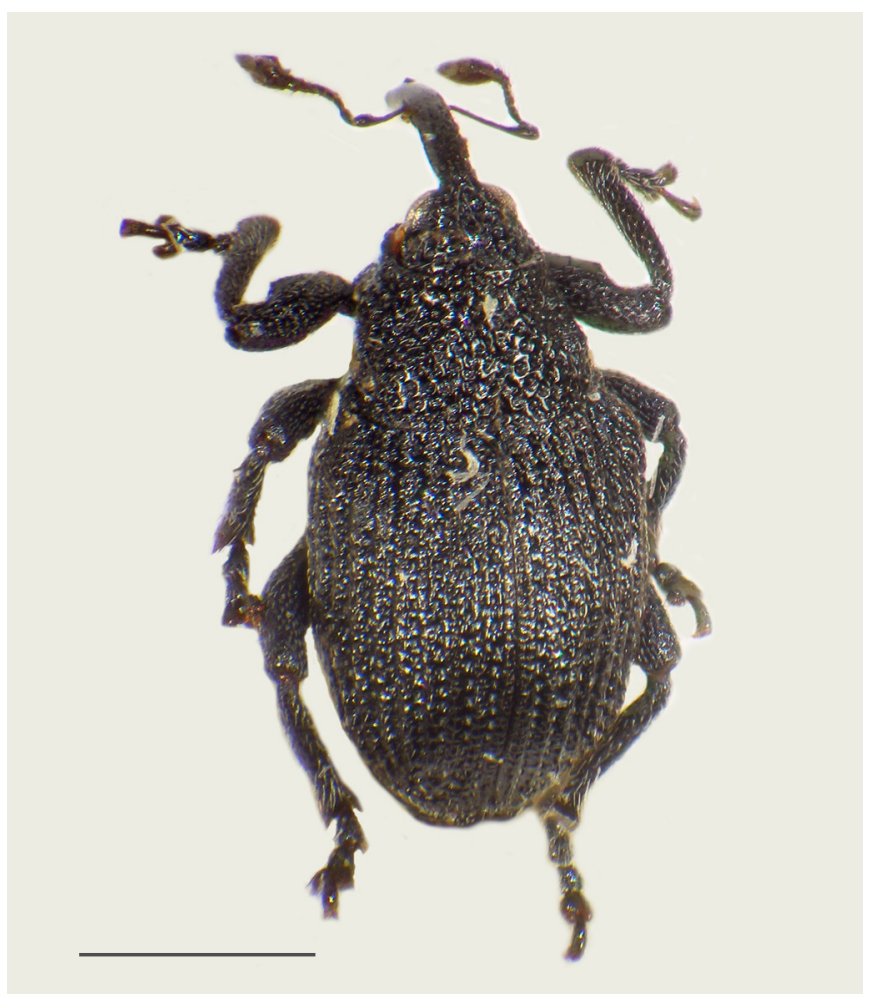

Fig. 14. Ceutorhynchus scrobicollis NerESHEIMER \& H. WAGNER, 1924 . Scale bar: $1 \mathrm{~mm}$. Photo Zoltán CsaTA

\section{Ceutorhynchus sulcicollis (PAYKULL, 1800)}

Published data: Montandon 1887, Fleck 1905

New data: Tulcea County: Slava Rusă, 4.V.2009, leg. JK; M-ţii Măcin, 5 km S Greci, 8.V.2017, leg. FK, KI, KJ, PA; Constanţa County: Furnica, Rezervaţia Dumbrăveni, 13-14.V.1995, leg. CP (Coll. Mus. G. AnTIPA) - Băneasa, Canaraua Fetii, 23.V.2015, leg. PA, RI, SzK, SzL.

\section{Ceutorhynchus syrites GERMAR, 1823}

New data: Tulcea County: S of Horia, 6.V.2009, leg. JK; Atmagea, 28.IV.2012, leg. KI; Enisala, 2425.V.2013 leg. KI, PA, RI, SzL, 29.IV-3.V.2014, leg. KI, IZ, PA, SzL; Slava Rusă, 24-25.V.2013, leg. KI, PA, RI, SzL; Babadag (forest), 30.IV.2014, leg. KI, IZ, PA, SzL; Slava Rusă, Uspenia Monastery, 11.V.2017, leg. FK, KI, KJ, PA; Codru Monastery, 10-11.V.2017, leg. FK, KI, KJ, PA; $2,7 \mathrm{~km}$ from Horia to S, 28.VI.2017, leg. KI; Constanţa County: Gura Dobrogei, 30.IV-2.V.2014, leg. KI, IZ, PA, SzL; Cheile Dobrogei, 2.V.2014, leg. KI, IZ, PA, SzL.

\section{*Ceutorhynchus talickyi KOROTYAeV, 1980}

New data: Tulcea County: S of Horia, 6.V.2009, leg. JK; Constanţa County: $3 \mathrm{~km} \mathrm{~W}$ of Crucea, Allah Bair (Nature Reserve), 1.V.2009, leg. JK.

\section{Ceutorhynchus turbatus SCHULTZE, 1903}

New data: Tulcea County: Codru env., 3.V.2009, leg. JK; Codru Monastery, 23-25.V.2013, leg. KI, PA, RI, SzL, 10-11.V.2017, leg. FK, KI, KJ, PA; Enisala, 24-25.V.2013, leg. KI, PA, RI, SzL, 29.IV-3.V.2014, leg. KI, IZ, PA, SzL; Slava Rusă, 24-25.V.2013, leg.
KI, PA, RI, SzL, 1-3.V.2014 leg. KI, IZ, PA, SzL; Enisala (castle), 21.V.2015, leg. PA, RI, SzK, SzL; Constanţa County: Furnica, Rezervaţia Dumbrăveni, 13-14.V.1995, leg. CP (Coll. Mus. G. AnTIPA) Cheile Dobrogei, 2.V.2014, leg. KI, IZ, PA, SzL; Palazu Mic, 22.V.2015, leg. PA, RI, SzK, SzL; Murfatlar, Fântâniţa, 22.V.2015, leg. PA, RI, SzK, SzL; Cochirleni, 3.IV.2016, leg. FK, KI, PA, SzL; 23 August, 17.IX.2016, leg. KI; Costineşti (coast), 17.IX.2016, leg. KI; Amzacea, 14.V.2017, leg. KI.

Ceutorhynchus typhae (HERBST, 1795) (= floralis PAYKULL, 1792)

Published data: JAQUET 1899a, 1899b, 1900c; NegrU and RoşCa 1967; Raduta 2014

New data: Tulcea County: Slava Rusă, Uspenia Monastery, 11.V.2017, leg. FK, KI, KJ, PA; Ciucurova, 3.VI.2018, leg. FK, KI, KJ, PA; Constanţa County: Furnica, Rezervaţia Dumbrăveni, 13-14.V.1995, leg. CP (Coll. Mus. G. ANTIPA) - Băneasa, Canaraua Fetii, 23.V.2015, leg. PA, RI, SzK, SzL; Gura Dobrogei, 1.IV.2016, leg. FK, KI, PA, SzL.

Ceutorhynchus unguicularis C. G. THOMson, 1871 New data: Tulcea County: Atmagea, 28.IV.2012, leg. KI; M-ţii Măcin, to the Peak Ţuţuiatu, 28.IV.2010, leg. TLA.

*Ceutorhynchus varius REY, 1895

New data: Constanța County: Eschioi, rez. Pădurea Eschioi, 2-3.VI.2013, leg. RS̆; M-ţii Măcin, Greci env., 6.V.2009, leg. JK.

Ceutorhynchus viridanus GYLLENHAL, 1837

New data: Tulcea County: Letea, 7-8.VI.1977, leg. AM (Coll. Mus. R. ANGHELUŢă).

Coeliastes lamii (FABRICIUS, 1792)

New data: Tulcea County: Luncaviţa, Valea Fagilor, 23.V.1997, leg. CH (Coll. Mus. G. ANTIPA)-Atmagea, 28.IV.2012, leg. KI; Slava Rusă, 1-3.V.2014, leg. KI, IZ, PA, SzL, 20.V.2015, leg. PA, RI, SzK, SzL, 11.V.2017, leg. FK, KI, KJ, PA; Caugagia, 1.V.2014, leg. KI, IZ, PA, SzL; Slava Rusă, Uspenia Monastery, 11.V.2017, leg. FK, KI, KJ, PA; 2,7 km from Horia to S, 28.VI.2017, leg. KI, KJ, PA, TB; Constanţa County: Gura Dobrogei, 28-29.IV.2012, leg. KI, 30.IV-2.V.2014, leg. KI, IZ, PA, SzL, 1.IV.2016, leg. FK, KI, PA, SzL; Băneasa, Canaraua Fetii (quarry), 23.V.2015, leg. PA, RI, SzK, SzL, 2-3.IV.2016, leg. FK, KI, PA, SzL.

Coeliodes rana (FABRICIUS, 1787) (= dryados GMELIN, 1790; = quercus FABRICIUs, 1787)

New data: Tulcea County: Slava Rusă, 11.V.2017, leg. FK, KI, KJ, PA.

Coeliodes transversealbofasciatus (GoEzE, 1777) (= cinctus GeOFFroY, 1785; = erythroleucos GMelin, 
1790)

New data: Tulcea County: Slava Rusă, 1-3.V.2014, leg. KI, IZ, PA, SzL.

Coeliodes trifasciatus BACH, 1854

New data: Constanţa County: Negru Vodă (Nature Reserve), 25.IX.2015, leg. KI, KJ, PA.

Datonychus arquatus (HERBST, 1795)

New data: Tulcea County: Smârdan, Braţul Măcin, 23.VI.2017, leg. KI, 2.VI.2018, leg. FK, KI, KJ, PA.

Datonychus melanostictus (MARSHAM, 1802)

New data: Tulcea County: Izvoarele, 21.V.1997, leg. CP (Coll. Mus. G. AnTIPA).

\section{Datonychus urticae (BOHEMAN, 1845)}

New data: Constanţa County: Gura Dobrogei, 1.IV.2016, leg. FK, KI, PA, SzL.

\section{Ethelcus denticulatus (SCHRANK, 1781)}

Published data: JAQUET 1900b, FLECK 1905, Montandon 1908, Negru and Roşca 1967, Raduta 2014

New data: Tulcea County: W of Horia, 6.V.2009, leg. JK; M-ţii Măcin, to the Peak Ţuţuiatu, 28.IV.2010, leg. TLA; Constanţa County: Esechioi (forest) 7.VII.2014, leg. KI; Gura Dobrogei, 7.VI.2018, leg. KI.

Glocianus albovittatus (GERMAR, 1823)

New data: Tulcea County: Enisala, 24-25.V.2013, leg. KI, PA, RI, SzL; W of Horia, 6.V.2009, leg. JK; Constanţa County: Pecineaga (forest), 14.V.2017, leg. FK, KI, KJ, PA.

Glocianus distinctus (C. N. F. BRISOUT de BARNEVILLE, 1870)

Published data: Montandon 1887, FleCK 1905

New data: Tulcea County: N from Nifon, 23.VI.2017, leg. KI; Constanţa County: Gura Dobrogei, 30.IV2.V.2014, leg. KI, IZ, PA, SzL; Esechioi (forest), 7.VII.2014, leg. KI, OA, PA, SzL.

Glocianus fennicus (FAUST, 1895)

New data: Tulcea County: Slava Rusă, Uspenia Monastery, 11.V.2017, leg. FK, KI, KJ, PA; Constanţa County: $3 \mathrm{~km}$ W of Crucea, Allah Bair (Nature Reserve), 1.V.2009, leg. JK; Gura Dobrogei, 12.V.2017, leg. FK, KI, KJ, PA.

Glocianus cf. polystriatus (SCHULTZE, 1898)

New data: Constanţa County: $3 \mathrm{~km}$ W of Crucea, Allah Bair (Nature Reserve), 1-2.VI.2008, leg. SR; $3 \mathrm{~km} \mathrm{~W}$ of Crucea, Allah Bair (Nature Reserve), 1.V.2009, leg. JK.

Glocianus punctiger (C. R. SAHLBERG, 1835)

New data: Tulcea County: M-ţii Măcin, Greci,
7.V.2017, leg. FK, KI, KJ, PA; Slava Rusă, Uspenia Monastery, 11.V.2017, leg. FK, KI, KJ, PA.

Hadroplontus litura (FABRICIUS, 1775)

New data: Tulcea County: Codru Monastery, 1011.V.2017, leg. KI; Horea, 3.VI.2018, leg. KI.

Hadroplontus trimaculatus (FABRICIUS, 1775)

New data: Tulcea County: Nifon, pârâul Pârlita valley, 23.VI.2017, leg. KI, KJ, PA, TB; Smârdan, Braţul Măcin, 18.VI.2019, leg. KI; Constanţa County: Furnica, Rezervaţia Dumbrăveni, 15.V.1995, leg. MA (Coll. Mus. G. AntiPA) - Pecineaga (forest), 14.V.2017, leg. FK, KI, KJ, PA.

*Microplontus millefolii (SCHUltze, 1897)

New data: Tulcea County: Smârdan, Braţul Măcin, 22.VI.2017, leg. KI, KJ, PA, TB.

Microplontus rugulosus (HERBST, 1795)

New data: Atmagea, 28.IV.2012, leg. KI.

Mogulones albosignatus (GYLLENHAL, 1837)

New data: Tulcea County: Enisala, 24-25.V.2013, leg. KI, PA, RI, SzL; Slava Rusă, 1-3.V.2014, leg. KI, IZ, PA, SzL.

Mogulones asperifoliarum (GYLLENHAL, 1813)

New data: Tulcea County: Slava Rusă, 25.V.2013, leg. KI, PA, RI, SzL, 1-3.V.2014, leg. KI, IZ, PA, SzL, 20.V.2015, leg. PA, RI, SzK, SzL, 9.V.2017, leg. FK, KI, KJ, PA, 11.V.2017, leg. FK, KI, KJ, PA; Constanţa County: Gura Dobrogei, 12.V.2017, leg. FK, KI, KJ, PA.

Mogulones austriacus (H. BRISOUT DE BARNEVILLE, 1869)

New data: Tulcea County: Enisala, 29.IV-3.V.2014, leg. KI, IZ, PA, SzL.

Mogulones borraginis (FABRICIUS, 1792)

New data: Constanţa County: Hagieni, Pădurea Hagieni (forest), 13.V.2017, leg. FK, KI, KJ, PA; Pecineaga (forest), 14.V.2017, leg. FK, KI, KJ, PA.

Mogulones crucifer (PALLAS, 1771)

New data: Tulcea County: M-ţii Măcin, Greci, 7.V.2017, leg. KI; Nifon, pârâul Pârlita valley, 23.VI.2017, leg. KI, KJ, PA, TB.

Mogulones diecki (C. N. F. BRISOUT de BARNEVILle, 1870) (= curvistriatus SCHULTZE, 1897)

New data: Tulcea County: Smârdan, Braţul Măcin, 2.VI.2018, leg. KI.

\section{Mogulones dimidiatus (I. FRIVALDSZKY, 1865)}

New data: Tulcea County: Slava Rusă, 31.III.2016, leg. FK, KI, PA, SzL; Atmagea env., 24.IX.2010, leg. JK. 
Mogulones euphorbiae (C. N. F. BRISOUT de BARNEVILLE, 1866)

New data: Constanţa County: Gura Dobrogei, 1.IV.2016, leg. FK, KI, PA, SzL.

\section{Mogulones geographicus (GOEZE, 1777)}

New data: Tulcea County: Atmagea env., 24.IX.2010, leg. JK; M-ţii Măcin, 5 km S Greci, 8.V.2017, leg. FK, KI, KJ, PA.

\section{Mogulones javetii (GERHARDT, 1867)}

New data: Tulcea County: Nifon, pârâul Pârlita valley, 23.VI.2017, leg. KI, KJ, PA, TB; General Praporgescu, 3.VI.2018, leg. KI.

\section{Mogulones korbi (SCHULTZE, 1901)}

New data: Constanţa County: $3,5 \mathrm{~km} \mathrm{SW}$ of Rasova, Lacul Baciului (lake), 2010.VI.1, leg. PJ.

\section{Mogulones raphani (FABRICIUS, 1792)}

New data: Tulcea County: Grindul Letea, Haşmacul lui Omer, Delta Dunării, 10.VI.1991, leg. DR (Coll. Mus. G. Antipa).

\section{Nedyus quadrimaculatus (LINNÉ, 1758)}

Published data: MonTANDON 1908

New data: Tulcea County: Luncaviţa, Valea Fagilor, 23. 05. 1997, leg. CH, CP; Celic Dere, 24. 05. 1997, leg. CH (Coll. Mus. G. ANTIPA) - Agighiol, 12.VI.1993, leg. TLA; M-ţii Măcin, Valea Fagilor, 30.V.2005, leg. CsS; Atmagea, 23.IV.2009, leg. TLA, 28.IV.2012, leg. KI; Măcin, Rezervaţia de la Greci, 23.IV.2009, leg. TLA; Slava Rusă, 1-3.V.2014, leg. KI, IZ, PA, SzL, 20.V.2015, leg. PA, RI, SzK, SzL, 31.III.2016, leg. FK, KI, PA, SzL, 9.V.2017, leg. FK, KI, KJ, PA, 11.V.2017, leg. FK, KI, KJ, PA; Caugagia, 1.V.2014, leg. KI, IZ, PA, SzL; Slava Rusă, Uspenia Monastery, 11.V.2017, leg. FK, KI, KJ, PA; Nifon, pârâul Pârlita valley, 23.VI.2017, leg. KI, KJ, PA, TB; $2,5 \mathrm{~km}$ from Stejaru to NW, 25.VI.2017, leg. KI, KJ, PA, TB; Constanţa County: Furnica, Rezervaţia Dumbrăveni, 13-14.V.1995, leg. CP; Negureni, 25.VI.1995, leg. CP, 21.VI.1996, leg. CP; Băneasa, Canaraua Fetii, 10.IV.1999, leg. SSP (Coll. Mus. G. ANTIPA) - Gura Dobrogei, 28-29.IV.2012, leg. KI, 26.V.2013, leg. KI, PA, RI, SzL, 1.IV.2016, leg. FK, KI, PA, SzL, 7.VI.2018, leg. FK, KI, KJ, PA; Murfatlar, Fântâniţa, 4-5.VII.2014, leg. KI, OA, PA, SzL; Băneasa, Canaraua Fetii (quarry), 7.VII.2014, leg. KI, OA, PA, SzL, 23.V.2015, leg. PA, RI, SzK, SzL, 2-3.IV.2016, leg. FK, KI, PA, SzL; Băneasa, Canaraua Fetii (forest), 15-17.IX.2016, leg. KI, KJ, PA, TB; Negureni, Pădurea Valea Cişmelelor, 23.V.2015, leg. PA, RI, SzK, SzL; Cochirleni, 3.IV.2016, leg. FK, KI, PA, SzL.

Oprohinus consputus (GERMAR, 1823)

New data: Tulcea County: Codru Monastery, 19.V.2015, leg. PA, RI, SzK, SzL; Slava Rusă, 11.V.2017, leg. FK, KI, KJ, PA; Constanţa County: Gura Dobrogei, 25-27.VI.2017, leg. KI, KJ, PA, TB,
7.VI.2018, leg. KI; Murfatlar, Fântâniţa, 7-8.VI.2018, leg. FK, KI, KJ, PA;

Oprohinus suturalis (FABRICIUS, 1775)

New data: Tulcea County: Slava Rusă, 11.V.2017, leg. FK, KI, KJ, PA.

\section{Parethelcus pollinarius (FORSTER, 1771)}

New data: Tulcea County: Pădurea Letea (forest), 17.VI.1993, leg. TLA; Atmagea, 23.IV.2009, leg. TLA, 28.IV.2012, leg. KI; Nifon, pârâul Pârlita valley, 23.VI.2017, leg. KI, KJ, PA, TB; Constanţa County: Păd. Hagieni, 22.V.1993, leg. CP; Furnica, Rezervaţia Dumbrăveni, 13-14.V.1995, leg. CP; Negureni, 25.VI.1995, leg. CP (Coll. Mus. G. ANTIPA) - Gura Dobrogei, 28-29.IV.2012, leg. KI, 7.VI.2018, leg. FK, KI, KJ, PA; Pădurea Hagieni (forest), 6.VII.2014, leg. KI, OA, PA, SzL; Băneasa, Canaraua Fetii (quarry), 2-3.IV.2016, leg. FK, KI, PA, SzL.

\section{Phrydiuchus topiarius (GERMAR, 1823)}

Published data: Montandon 1908, Negru and RoşCa 1967, RADUTA 2014

\section{Phrydiuchus tau WARNER, 1969}

New data: Constanţa County: Agigea, 20.VI.1964, leg. IC; Furnica, Rezervaţia Dumbrăveni, 22.VI.1995, leg. CP (Coll. Mus. G. ANTIPA) - Cheia, Nature Reserve, 2 $\mathrm{km} \mathrm{S}$ of Cheia, 2-3.VI.2008, leg. PJ.

\section{Poophagus sisymbrii (FABRICIUS, 1777)}

New data: Tulcea County: Smârdan, Brațul Măcin, 22.VI.2017, leg. KI, 2.VI.2018, leg. FK, KI, KJ, PA.

Prisistus obsoletus (GERMAR, 1823)

Published data: JAQUET 1899b, FleCK 1905

New data: Tulcea County: Măcin (hillside, 200 m), 29.V.2005, leg. CsS; M-ţii Măcin, Pricopan, 1.VI.2005, leg. CsS.

*Ranunculiphilus (Austroceutorhynchus) italicus (C. N. F. Brisout de BARNEville, 1869)

New data: Tulcea County: Turcoaia, $6 \mathrm{~km}$ from Cerna to NW, 3.VI.2018, leg. FK, KI, KJ, PA; Constanța County: Limanu env., 2.VI.2010, leg. RŠ; $1,5 \mathrm{~km}$ E of Cotu Văii, 3.VI.2013, leg. JP; Palazu Mic, 22.V.2015, leg. PA.

\section{Ranunculiphilus (Ranunculiphilus) faeculentus GyLlenHAL, 1837 \\ New data: Tulcea County: 2,5 km from Stejaru to NW, 25.VI.2017, leg. KI, KJ, PA, TB; $2 \mathrm{~km}$ from Slava Rusă to NE, 3.VI.2018, leg. FK, KI, KJ, PA; General Praporgescu, 3.VI.2018, leg. KI; M-ţii Măcin, Greci, 18-19.VI.2019, leg. KI, KJ, PA, TB; Constanţa County: Agigea, 27.V.1963, leg. XS (Coll. Mus. G. AnTIPA) - Gura Dobrogei, 7.VI.2018, leg. KI.}

\section{Sirocalodes depressicollis (GYLLENHAL, 1813)}


New data: Constanţa County: Gura Dobrogei, 30.IV2.V.2014, leg. KI, IZ, PA, SzL; Palazu Mic, 22.V.2015, leg. PA, RI, SzK, SzL; Pecineaga (forest), 14.V.2017, leg. FK, KI, KJ, PA.

\section{Stenocarus cardui (Herbst, 1784)}

New data: Tulcea County: Stejaru env., 5.V.2009, leg. JK; M-ţii Măcinu, Greci env., 6.V.2009, leg. JK; Codru Monastery, 23-25.V.2013, leg. KI, PA, RI, SzL, 24.VI.2017, leg. KI, KJ, PA, TB, 10-11.V.2017, leg. FK, KI, KJ, PA, 4.VI.2018, leg. KI; Enisala, 24-25.V.2013, leg. KI, PA, RI, SzL; Slava Rusă, 1-3.V.2014, leg. KI, IZ, PA, SzL, 20.V.2015, leg. PA, RI, SzK, SzL, 31.III.2016, leg. FK, KI, PA, SzL; M-ţii Măcin, 5 km S Greci, 8.V.2017, leg. FK, KI, KJ, PA; Slava Rusă, Uspenia Monastery, 11.V.2017, leg. FK, KI, KJ, PA; N from Nifon, 23.VI.2017, leg. KI, KJ, PA, TB; M-ţii Măcin, Nifon (forest), 23.VI.2017, leg. KI, KJ, PA, TB; $1,5 \mathrm{~km}$ from Beidaud to SW, 25.VI.2017, leg. KI, KJ, PA, TB; 2,5 km from Stejaru to NW, 25.VI.2017, leg. KI, KJ, PA, TB; Nifon, pârâul Pârlita valley, 23.VI.2017, leg. TB; Smârdan, Braţul Măcin, 7.V.2017, leg. FK, KI, KJ, PA; 2 km from Slava Rusă to NE, 3.VI.2018, leg. FK, KI, KJ, PA; Enisala (castle), 20.VI.2019, leg. KI, KJ, PA, TB; Constanţa County: Furnica, Rezervaţia Dumbrăveni, 13-14.V.1995, leg. CP, 22.VI.1995, leg. CP (Coll. Mus. G. ANTIPA) - Gura Dobrogei, 28 29.IV.2012, leg. KI, 26.V.2013, leg. KI, PA, RI, SzL, 12.V.2017, leg. FK, KI, KJ, PA, 27.VI.2017, leg. KI, KJ, PA, TB, 7.VI.2018, leg. FK, KI, KJ, PA; Cheile Dobrogei, 2.V.2014, leg. KI, IZ, PA, SzL; Murfatlar, Fântâniţa, 4-5.VII.2014, leg. KI, OA, PA，SzL, 22.V.2015, leg. PA, RI, SzK, SzL, 24-25.IX.2015, leg. KI, KJ, PA, 7-8.VI.2018, leg. FK, KI, KJ, PA; Pădurea Hagieni (forest), 6.VII.2014, leg. KI, OA, PA, SzL, 12.V.2017, leg. FK, KI, KJ, PA; Esechioi (forest), 7.VII.2014, leg. KI, OA, PA, SzL; Băneasa, Canaraua Fetii, 23.V.2015, leg. PA, RI, SzK, SzL; Negru Vodă (Nature Reserve), 25.IX.2015, leg. KI, KJ, PA; Pecineaga (forest), 14.V.2017, leg. FK, KI, KJ, PA; Istria, Cetatea Histria, 26-28.VI.2017, leg. KI, KJ, PA, TB; $2 \mathrm{~km}$ from Sinoie to SE, 27.VI.2017, leg. KI, KJ, PA, TB, 5.VI.2018, leg. FK, KI, KJ, PA; 2,7 km from Horia to S, 28.VI.2017, leg. KI, KJ, PA, TB; Cobadin, 14.V.2017, leg. FK, KI, KJ, PA; Sinoie, Grindul Lupilor, 5.VI.2018, leg. FK, KI, KJ, PA; Crucea, 9.VI.2018, leg. FK, KI, KJ, PA.

\section{Stenocarus ruficornis (STEPHENS, 1831)}

New data: Constanţa County: Vadu, Grindul Chituc, 6.VI.2018, leg. FK, KI, KJ, PA.

\section{*Thamiocolus roubali DIECKMANN, 1973}

New data: Tulcea County: Codru env., 9-10.VI.2005, leg. RS , (compared with type); Constanţa County: 3 km W of Crucea, Allah Bair (Nature Reserve), 3-4. VI.2010, leg. R $\breve{S}$, JP.
Thamiocolus signatus (GYLlENHAL, 1837)

Published data: JAQUET 1899a, 1904; FLECK 1905

New data: Tulcea County: Slava Rusă, 11.V.2017, leg. KI; Constanţa County: Murfatlar, Fântâniţa, 22.V.2015, leg. PA, RI, SzK, SzL.

*Thamiocolus sinapis (DeSBrochers des Loges, 1893)

New data: Tulcea County: $6 \mathrm{~km}$ W of Somova, 8.VI.2008, leg. PJ; M-ţii Măcin, 4,5 km SE of Greci, 8-9.VI.2008, leg. SL.

\section{Trichosirocalus horridus (PANZER, 1801)}

New data: Tulcea County: Turcoaia, $6 \mathrm{~km}$ from Cerna to NW, 24.VI.2017, leg. KI, KJ, PA, TB; Ciucurova, 3.VI.2018, leg. FK, KI, KJ, PA; Enisala (wooded pasture), 4.VI.2018, leg. FK, KI, KJ, PA; Enisala (castle), 20.VI.2019, leg. KI; Constanţa County: Gura Dobrogei, 28-29.IV.2012, leg. KI; Sinoie, Grindul Lupilor, 5.VI.2018, leg. FK, KI, KJ, PA, 22.VI.2019, leg. KI, KJ, PA, TB.

\section{Trichosirocalus troglodytes (FABRICIUS, 1787)}

New data: Tulcea County: M-ţii Măcin, Valea Fagilor (quarry), 30.V.2005, leg. CsS; Enisala (castle), 20.VI.2019, leg. KI, KJ, PA, TB; Constanţa County: Agigea, 27.V.1963, leg. XS (Coll. Mus. G. ANTIPA) $2 \mathrm{~km}$ from Sinoie to SE, 27.VI.2017, leg. KI, KJ, PA, TB; Sinoie, Grindul Lupilor, 5.VI.2018, leg. KI.

\section{Zacladus asperatus (GYLLENHAL, 1837)}

New data: Tulcea County: Babadag (forest), 30.IV.2014, leg. KI, IZ, PA, SzL; Slava Rusă, 1-3.V.2014, leg. KI, IZ, PA, SzL; Constanţa County: Istria env., Cetatea Histria, 2010.IX.22, leg. JK; Gura Dobrogei, 30.IV-2.V.2014, leg. KI, IZ, PA, SzL; Murfatlar, Fântâniţa, 4-5.VII.2014, leg. KI, OA, PA, SzL, 22.V.2015, leg. PA, RI, SzK, SzL; Băneasa, Canaraua Fetii, 23.V.2015, leg. PA, RI, SzK, SzL; Sinoie, Grindul Lupilor, 27.VI.2017, leg. KI, KJ, PA, TB, 5.VI.2018, leg. FK, KI, KJ, PA; Vadu, Grindul Chituc, 6.VI.2018, leg. FK, KI, KJ, PA.

Zacladus exiguus (OLIVIER, 1807)

Published data: TeOdor and Manole 1996, Raduta 2014

New data: Tulcea County: Pădurea Letea (forest), 17.VI.1993, leg. TLA; Slava Rusă, 24-25.V.2013, leg. KI, PA, RI, SzL; Constanţa County: Gura Dobrogei, 30.IV-2.V.2014, leg. KI, IZ, PA, SzL.

\section{Zacladus geranii (PAYKULL, 1800) \\ Published data: JAQUET $1902 \mathrm{~b}$}

\section{Mononychini LeConte, 1876}

Mononychus punctumalbum (HERBST, 1784)

New data: Tulcea County: Caraorman, 8.V.1967, leg. XS, 9.V.1967, leg. MW, 10.V.1967, leg. MW, 2.V.1968, leg. XP, 13.V.1968, leg. XS, 15.V.1992, leg. 
NG; Maliuc, Mila 26, 18-19.V.1992, leg. IM (Coll. Mus. G. ANTIPA) - Smârdan, Braţul Măcin, 7.V.2017, leg. FK, KI, KJ, PA, 22.VI.2017, leg. KI, 2.VI.2018, leg. FK, KI, KJ, PA.

Phytobiini Gistel, 1848

Neophytobius granatus (GYLLENHAL, 1835)

New data: Tulcea County: Atmagea, 28.IV.2012, leg. $\mathrm{KI}$.

Pelenomus waltoni (BoHeMAN, 1843)

New data: Tulcea County: Smârdan, Braţul Măcin, 11.IX.2016, leg. KI, 22.VI.2017, leg. KI.

Phytobius leucogaster (MARSHAM, 1802)

New data: Tulcea County: Greci (village), 22.VI.2017, leg. KI, KJ, PA, TB; M-ţii Măcin, Greci, 18-19. VI.2019, leg. KI, KJ, PA, TB; Enisala, 20.VI.2019, leg. KI; Constanţa County: Sinoie, 23.VI.2019, leg. KI, KJ, PA, TB.

Rhinoncus albicinctus GYLLENHAL, 1837

New data: Tulcea County: Smârdan, Braţul Măcin, 21-23.IX.2015, leg. KI, 11.IX.2016, leg. KI, KJ, PA, TB.

Rhinoncus bruchoides (HERBST,1784)

New data: Tulcea County: Smârdan, Braţul Măcin, 28.VI.2017, leg. KI, KJ, PA, TB.

Rhinoncus inconspectus (HERBST, 1795)

(= gramineus FABRICIUS, 1792)

New data: Tulcea County: Parcul Naţional M-ţii Măcin, Culmea Pricopanului, 31.VII.2006, leg. EP (Coll. Mus. G. ANTIPA) - Smârdan, Braţul Măcin, 22.VI.2017, leg. KI, KJ, PA, TB, 2.VI.2018, leg. FK, KI, KJ, PA.

Rhinoncus leucostigma (MARSHAM, 1802)

(= pericarpius PAYKULL, 1792)

New data: Tulcea County: Smârdan, Braţul Măcin, 7.V.2017, leg. FK, KI, KJ, PA, 22-23.VI.2017, leg. KI; Slava Rusă, Uspenia Monastery, 11.V.2017, leg. FK, KI, KJ, PA; Nifon, pârâul Pârlita valley, 23.VI.2017, leg. KI, KJ, PA, TB; Ciucurova, 3.VI.2018, leg. FK, KI, KJ, PA; 2 km from Slava Rusă to NE, 3.VI.2018, leg. KI; Constanţa County: Furnica, Rezervaţia Dumbrăveni CT, 13-14.V.1995, leg. CP (Coll. Mus. G. ANTIPA) - Băneasa, Canaraua Fetii (quarry), 2-3. IV.2016, leg. FK, KI, PA, SzL; Băneasa, Canaraua Fetii (forest) 17.IX.2016, leg. KI, KJ, PA, TB; Hagieni, Pădurea Hagieni (forest), 13.V.2017, leg. KI.

Rhinoncus pericarpius (LINNÉ, 1758) (= castor Fabricius, 1792)

Published data: RADUTA 2014

New data: Constanţa County: Cochirleni, 3.IV.2016, leg. FK, KI, PA, SzL.
Rhinoncus perpendicularis (REICH, 1797)

Published data: MonTANDON 1908

New data: Tulcea County: Codru Monastery, 30.IV3.V.2014, leg. KI, IZ, PA, SzL; Smârdan, Braţul Măcin, 11.IX.2016, leg. KI, KJ, PA, TB, 7.V.2017, leg. FK, KI, KJ, PA, 22.VI.2017, leg. KI, KJ, PA, TB; Constanţa County: Gura Dobrogei, 26.V.2013, leg. KI, PA, RI, SzL; Cheile Dobrogei, 2.V.2014, leg. KI, IZ, PA, SzL; $2 \mathrm{~km}$ from Sinoie to SE, 5.VI.2018, leg. FK, KI, KJ, PA.

Scleropterini Schultze, 1902

Homorosoma validirostre (GYLLENHAL, 1837)

Published data: RADUTA 2014

Tapinotus sellatus (FABRICIUS, 1794)

New data: Tulcea County: C. A. Rosetti, Delta D., 7.VI.1981, leg. CP (Coll. Mus. G. ANTIPA).

CONODERITAE SCHÖNHERR, 1825

Coryssomerini C. G. Thomson, 1859

Coryssomerus capucinus (BECK, 1817)

Published data: Negru and Roşca 1967, Raduta 2014

New data: Tulcea County: Celic Dere, 20.V.1997, leg. CH (Coll. Mus. G. AntiPA) - Enisala, 29.IV3.V.2014, leg. KI, IZ, PA, SzL; Constanţa County: Hagieni, Pădurea Hagieni (forest), 17.IX.2016, leg. FK, KI, KJ, PA.

ORBITIDITAE C. G. ThOMSON, 1859

Orobitidini C. G. ThOMsON, 1859

Orobitis cyanea (LINNÉ, 1758)

New data: Tulcea County: Slava Rusă, 1-3.V.2014, leg. KI, IZ, PA, SzL.

COSSONINAE SCHÖNHERR, 1825

Cossonini SCHÖNHERR, 1825

Cossonus (Caenocossonus) cylindricus C. R. SAHLBERG, 1835

New data: Tulcea County: Caraorman, 16.VI.1969, leg. ŞN (Coll. Mus. G. AnTIPA).

Mesites (Mesites) cunipes BoHEMAN, 1838

Published data: MontANDON 1887, FLECK 1905

Mesites (Mesites) pallidipennis BOHEMAN, 1838

Published data: ProcheŞ 1999

Onycholipini Wollaston, 1873

Hexarthrum exiguum (BOHEMAN, 1838)

Published data: Montandon 1908, Negru 1968, IENIŞTEA 1974

CURCULIONINAE Latreille, 1802

Acentrusini Alonso-ZaraZAGA, 2005

Acentrus histrio (SCHÖNHERR, 1837)

New data: Constanţa County: Mangalia, Pădurea Comorova, 2.VI.1962, leg. APG; Hagieni, 6.VI.1965, 
leg. MW (Coll. Mus. G. ANTIPA).

Anthonomini C. G. Thomson, 1859

Anthonomus (Anthonomus) chevrolati chevrolati DesBrochers des LoGes, 1868

New data: Constanţa County: Gura Dobrogei, 26.V.2013, leg. KI, PA, RI, SzL.

Anthonomus (Anthonomus) incurvus (PANZER, 1795) (= humeralis PANZER, 1795)

New data: Tulcea County: M-ţii Măcin, to the Peak Ţuţuiatu, 28.IV.2010, leg. TLA; Constanţa County: Pădurea Hagieni (forest), 6.VII.2014, leg. KI, OA, PA, SzL.

\section{Anthonomus (Anthonomus) pedicularius (LINNÉ, 1758)}

New data: Tulcea County: Babadag, Visterna, 15.V.1963, leg. IC (Coll. Mus. G. ANTIPA) - 2,7 km from Horia to S, 28.VI.2017, leg. KI, KJ, PA, TB; Turcoaia, $6 \mathrm{~km}$ from Cerna to NW, 19.VI.2019, leg. KI, KJ, PA, TB; Constanţa County: Esechioi (forest), 1-2.IV.2016, leg. FK, KI, PA. SzL; Gura Dobrogei, 25-27.VI.2017, leg. TB; Murfatlar, Fântâniţa, 7-8. VI.2018, leg. KI.

\section{Anthonomus (Anthonomus) pomorum (LINNÉ, 1758)}

New data: Tulcea County: Slava Rusă, 9.V.2017, leg. FK, KI, KJ, PA; M-ţii Măcin, 5 km S Greci, 8.V.2017, leg. FK, KI, KJ, PA; Constanţa County: Murfatlar, Fântâniţa, 22.V.2015, leg. PA, RI, SzK, SzL; Băneasa, Canaraua Fetii (quarry), 2-3.IV.2016, leg. FK, KI, PA. SzL; Albeşti, Pădurea Hagieni (forest), 12.V.2017, leg. FK, KI, KJ, PA.

\section{Anthonomus (Anthonomus) rubi (HERBST, 1795)}

New data: Tulcea County: Delta Dunării-G. Babarada-Şontea, 30.VII.2004, leg. GP (Coll. Mus. R. ANGHELuŢĂ) - Olguţa, 11.V.2007, leg. NJ, RE, SoL, VJ; Sarichioi, 16.07.2009, leg. EI (Coll. Mus. G. Antipa) - Pădurea Letea (forest), 17.VI.1993, leg. TLA; Smârdan, Braţul Măcin, 22.VI.2017, leg. KI, KJ, PA, TB; Slava Rusă, 9.V.2017, leg. KI; Constanţa County: Pădurea Hagieni (forest), 6.VII.2014, leg. KI, OA, PA, SzL.

\section{Anthonomus}

(Gyllenhal, 1835)

(Anthonomidius)

rubripes

New data: Tulcea County: Greci, 23-27.V.1961, leg. MW; Celic Dere, 20.V.1997, leg. CH (Coll. Mus. G. ANTIPA) - Frecăței, Celic Dere Monastery (hillside) 31.V.2005, leg. CsS; Stejaru env., 5.V.2009, leg. JK.

\section{Anthonomus ulmi (DeGEer, 1775)}

New data: Tulcea County: Codru Monastery, 30-31. III.2016, leg. FK, KI, PA, SzL; Constanţa County: Băneasa, Canaraua Fetii (forest), 15-17.IX.2016, leg. KI, KJ, PA, TB; Esechioi (forest), 1-2.IV.2016, leg.
FK, KI, PA. SzL.

Bradybatus (Bradybatus) creutzeri GERMAR, 1823

Published data: Negru 1957, Negru and RoşCa 1967, RADUTA 2014

New data: Tulcea County: Slava Rusă, 23.IV.2009, leg. TLA; M-ţii Măcin, to the Peak Ţuţuiatu, 28.IV.2010, leg. TLA.

Bradybatus (Bradybatus) seriesetosus PETRI, 1912

New data: Tulcea County: Stejaru env., 5.V.2009, leg. JK; Babadag (forest), 23-25.V.2013, leg. KI, PA, RI, SzL; Slava Rusă, 1-3.V.2014, leg. KI, IZ, PA, SzL; M-ţii Măcin, Greci, 8.V.2017, leg. FK, KI, KJ, PA; Slava Rusă, Uspenia Monastery, 11.V.2017, leg. FK, KI, KJ, PA; Enisala, 10.V.2017, leg. FK, KI, KJ, PA; M-ţii Măcin, Nifon (forest), 23.VI.2017, leg. KI, KJ, PA, TB; 2,5 km from Stejaru to NW, 25.VI.2017, leg. KI, KJ, PA, TB; Constanţa County: Furnica, Rezervaţia Dumbrăveni, 13-14.V.1995, leg. CP (Coll. Mus. G. ANTIPA) - Gura Dobrogei, 30.IV-2.V.2014, leg. KI, IZ, PA, SzL; Băneasa, Canaraua Fetii (forest), 23.V.2015, leg. PA, RI, SzK, SzL, 15-17.IX.2016, leg. KI, KJ, PA, TB; Negru Vodă (Nature Reserve) 25.IX.2015, leg. KI, KJ, PA; Neptun-Olimp, Pădurea Comorova, 13.V.2017, leg. FK, KI, KJ, PA.

Bradybatus (Bradybatus) tomentosus DESBROCHERS DES LOGES, 1892

New data: Tulcea County: Slava Rusă, 1-3.V.2014, leg. KI, IZ, PA, SzL.

\section{CiOnini SCHÖNHERR, 1825}

\section{Cionus clairvillei BOHEMAN, 1838}

New data: Tulcea County: M-ţii Măcin, Greci (400 m), 13.VI.2004, leg. GP; Cheile Dobrogei (170200 m), 14.VI.2004, leg. GP; M-ţii Măcin, Drumul Vinului, 30.VIII.2007, leg. CI; M-ţii Măcin, Creasta Cardonului, 30.VIII.2007, leg. CI (Coll. Mus. R. ANGHeluță) - $3 \mathrm{~km}$ from Slava Rusă to NE, 24.VI.2017, leg. KI; S from Hamcearca, 24.VI.2017, leg. KI; 2 km from Slava Rusă to NE, 3.VI.2018, leg. KI.

\section{Cionus hortulanus (GEOFFROY, 1785)}

Published data: FleCK 1905, Montandon 1908

New data: Tulcea County: Mănăstirea Cocoş, 10.VI.1973, leg. AC; Măcin, Valea Suluc, 27.V.2005, leg. IM; Mănăstirea Cocoş, 24.V.2005, leg. CC (Coll. Mus. G. Antipa) - M-ţii Măcin, Valea Fagilor (quarry), 30.V.2005, leg. CsS; Codru Monastery, 23-25.V.2013, leg. KI, PA, RI, SzL, 25.VI.2017, leg. KI, KJ, PA, TB, 20.VI.2019, leg. KI, KJ, PA, TB; Slava Rusă, 24-25.V.2013, leg. KI, PA, RI, SzL; Nifon, pârâul Pârlita valley, 23.VI.2017, leg. KI, KJ, PA, TB; N from Nifon, 23.VI.2017, leg. TB; S from Hamcearca, 24.VI.2017, leg. KI, KJ, PA, TB; 2 km from Slava Rusă to NE, 3.VI.2018, leg. FK, KI, KJ, PA; M-ţii Măcin, Greci, 18-19.VI.2019, leg. KI, KJ, 
PA, TB; Enisala, 20.VI.2019, leg. KI; Enisala (castle), 20.VI.2019, leg. KI, KJ, PA, TB; Constanţa County: Hagieni, 29.V.1963, leg. NS; Furnica, Rezervaţia Dumbrăveni, 22.VI.1995, leg. CP (Coll. Mus. G. ANTIPA) - Gura Dobrogei, 28-29.IV.2012, leg. KI; Murfatlar, Fântâniţa, 4-5.VII.2014, leg. KI, OA, PA, SzL; Esechioi (forest), 7.VII.2014, leg. KI, OA, PA, SzL; Esechioi (semi-dry slope steppic grassland with shrub encroachment), 15.IX.2016, leg. KI, KJ, PA, TB; Murfatlar, Fântâniţa, 24-25.IX.2015, leg. KI, KJ, PA; Băneasa, Canaraua Fetii (forest), 17.IX.2016, leg. KI, KJ, PA, TB; Vadu, Grindul Chituc, 22.VI.2019, leg. KI.

\section{Cionus olens (FABRICIUS, 1792)}

Published data: Montandon 1908

New data: Tulcea County: Atmagea env., 24.IX.2010, leg. JK; S from Hamcearca, 24.VI.2017, leg. KI; Constanţa County: Limanu, 12.V.2017, leg. FK, KI, KJ, PA.

\section{Cionus olivieri RoSENSCHÖLD, 1838}

Published data: HURMUZACHI 1904, FLECK 1905, Montandon 1908, Negru 1968, Teodor and MANOLE 1996, Raduta 2014

New data: Tulcea County: M-ţii Măcin, Greci (400 m), 13.VI.2004, leg. GP; M-ţii Măcin, Pricopan (300 m), 21.VI.2004, leg. AR; M-ţii Măcin, Drumul Vinului, 30.VIII.2007, leg. CI (Coll. Mus. R. Angheluță); Pădurea Letea, 20.VII.1966, leg. ŞN; C. A. Rosetti, Pădurea Letea, Delta Dunării, 26.V.1980, leg. APG; Măcin, Valea Suluc, 27.V.2005, leg. IM (Coll. Mus. G. ANTIPA) - Iancina, 11.VI.1993, leg. TLA; Periprava, 1.VII.2005, leg. DDC; Codru Monastery, 23-25.V.2013, leg. KI, PA, RI, SzL, 4.VI.2018, leg. KI, 20.VI.2019, leg. KI, KJ, PA, TB; Slava Rusă, 24-25.V.2013, leg. KI, PA, RI, SzL; 3 km from Slava Rusă to NE, 24.VI.2017, leg. TB; Turcoaia, 6 km from Cerna to NW, 24.VI.2017, leg. KI; $2 \mathrm{~km}$ from Slava Rusă to NE, 3.VI.2018, leg. KI; M-ţii Măcin, Greci, 18-19.VI.2019, leg. KI, KJ, PA, TB; Enisala (castle), 20.VI.2019, leg. KI, KJ, PA, TB; Constanţa County: Tuzla, leg. ALM (Coll. Mus. G. ANTIPA) Gura Dobrogei, 28-29.IV.2012, leg. KI; Negru Vodă (Nature Reserve) 25.IX.2015, leg. KI, KJ, PA.

\section{Cionus pulverosus pulverosus GUÉRIN-MÉNEVILLE,} 1838

New data: Tulcea County: Izvoarele, 21.V.1997, leg. RS (Coll. Mus. G. AnTIPA) - Codru Monastery, 2325.V.2013, leg. KI, PA, RI, SzL, 10-11.V.2017, leg. FK, KI, KJ, PA, 4.VI.2018, leg. KI; Slava Rusă, 2425.V.2013, leg. KI, PA, RI, SzL; M-ţii Măcin, Greci, 8.V.2017, leg. FK, KI, KJ, PA, 18-19.VI.2019, leg. KI, KJ, PA, TB; N from Nifon, 23.VI.2017, leg. TB; Nifon, pârâul Pârlita valley, 23.VI.2017, leg. KI, KJ, PA, TB; 2 km from Slava Rusă to NE, 3.VI.2018, leg. FK, KI, KJ, PA.
Cionus thapsus thapsus (FABRICIUS, 1792)

Published data: Montandon 1887, 1908; Fleck 1905 New data: Tulcea County: N from Nifon, 23.VI.2017, leg. TB; 2 km from Slava Rusă to NE, 3.VI.2018, leg. KI; M-ţii Măcin, Greci, 18-19.VI.2019, leg. KI, KJ, PA, TB; Codru Monastery, 20.VI.2019, leg. KI, KJ, PA, TB; Constanţa County: Esechioi (forest), 7.VII.2014, leg. KI, OA, PA, SzL; Vadu, Grindul Chituc, 22.VI.2019, leg. KI, KJ, PA, TB.

\section{Cionus tuberculosus (SCOPOLI, 1793) \\ Published data: Montandon 1908 \\ New data: Tulcea County: M-ţii Măcin, Nifon (forest), 23.VI.2017, leg. KI, KJ, PA, TB; S from Hamcearca, 24.VI.2017, leg. KI.}

\section{Cleopus pulchellus (HERBST, 1795) \\ Published data: FLECK 1905}

\section{Cleopus solani (FABRICIUS, 1792)}

New data: Constanţa County: Sinoie, Grindul Lupilor, 5.VI.2018, leg. FK, KI, KJ, PA.

\section{Stereonychus fraxini (DEGEER, 1775) \\ Published data: Negru 1968}

New data: Tulcea County: Periprava, 08.V.1964, leg. XS; C. A. Rosetti, Delta Dunării, 08.VI.1981, leg. CP (Coll. Mus. G. ANTIPA) - Pădurea Letea (forest), 17.VI.1993, leg. TLA; Măcin, Rezervaţia de la Greci, 23.IV.2009, leg. TLA; W of Horia, 6.V.2009, leg. JK; Babadag (forest), 23-25.V.2013, leg. KI, PA, RI, SzL, 30.IV.2014, leg. KI, IZ, PA, SzL; Slava Rusă, 24 25.V.2013, leg. KI, PA, RI, SzL, 1-3.V.2014, leg. KI, IZ, PA, SzL; Constanţa County: Limanu, 12.V.2017, leg. FK, KI, KJ, PA.

Curculionini Latreille, 1802

Archariina Pelsue \& O’Brien, 2011

Archarius (Archarius) pyrrhoceras (MARSHAM, 1802)

New data: Tulcea County: C. A. Rosetti, 1.VII.1950. (Coll. Mus. G. Antipa) - Beştepe, 13.VI.1993, leg. TLA; Slava Rusă, 24-25.V.2013, leg. KI, PA, RI, SzL, 1-3.V.2014, leg. KI, IZ, PA, SzL, 11.V.2017, leg. FK, KI, KJ, PA; Enisala, 29.IV-3.V.2014, leg. KI; Codru Monastery, 10.V.2017, leg. KI; Constanţa County: Pecineaga (forest), 14.V.2017, leg. FK, KI, $\mathrm{KJ}$, PA.

\section{Curculionina LATREILLE, 1802}

Curculio (Curculio) elephas (Gyllenhal, 1835)

Published data: TeOdor 1993, RAduta 2014

New data: Tulcea County: Canaraua Fetei, 17.X.1992, leg. SzL; M-ţii Măcin, Izvorul lui Moş Kalâm, 05.IX.2005, leg. CŞ (Coll. Mus. R. ANGHELUȚă).

Curculio (Curculio) glandium MARSHAM, 1802

New data: Tulcea County: M-ţii Măcin, Vf. Ţuţuiatu (400 m), 26.V.2006, leg. MC (Coll. Mus. R. 
ANGHELUȚă); Revărsarea, 23.V.1997, leg. CP (Coll. Mus. G. AnTIPA) - Babadag (forest), 2.VI.2005, leg. CsS, 22.IX.2015, leg. KI, KJ, PA, 13.IX.2016, leg. KI, KJ, PA, TB; Podişul Babadagului, 2007.V.17, leg. NJ, RE, SoL, VJ; Atmagea, 23.IV.2009, leg. TLA; Măcin, Rezervaţia de la Greci, 23.IV.2009, leg. TLA; Slava Rusă, 24-25.V.2013, leg. KI, PA, RI, SzL, 1-3.V.2014, leg. KI, IZ, PA, SzL, 20.V.2015, leg. PA, RI, SzK, SzL, 9.V.2017, leg. FK, KI, KJ, PA; Codru Monastery, 30.IV-3.V.2014, leg. KI, IZ, PA, SzL, 10.V.2017, leg. KI; Enisala, 29.IV-3.V.2014, leg. KI, IZ, PA, SzL; Caugagia, 1.V.2014, leg. KI, IZ, PA, SzL; M-ţii Măcin, 5 km S Greci, 8.V.2017, leg. FK, KI, KJ, PA; 2,5 km from Stejaru to NW, 25.VI.2017, leg. KI, KJ, PA, TB; 3 km from Slava Rusă to NE, 24.VI.2017, leg. KI; $2,7 \mathrm{~km}$ from Horia to S, 28.VI.2017, leg. KI, KJ, PA, TB; Constanţa County: Esechioi (forest), 7.VII.2014, leg. KI, OA, PA, SzL, 1-2.IV.2016, leg. FK, KI, PA, SzL, 16.IX.2016, leg. KI, KJ, PA, TB; Neptun-Olimp, Pădurea Comorova, 17.IX.2016, leg. FK, KI, KJ, PA; Pecineaga (forest), 14.V.2017, leg. FK, KI, KJ, PA.

\section{Curculio (Curculio) nucum LINNÉ, 1758}

Published data: Montandon 1887, 1908; FleCK 1905; TeODOR 1993; RAdUTA 2014

\section{Curculio (Curculio) pellitus (BOHEMAN, 1843)}

New data: Tulcea County: Podişul Babadagului, 17.V.2007, leg. NJ, RE, SoL, VJ; Horia-Praporgescu, 23.IV.2009, leg. TLA.

Curculio (Curculio) propinquus (DESBROCHERS des Loges, 1868)

New data: Tulcea County: Băneasa, Canaraua Fetii, 17.X.1992, leg. SzL (Coll. Mus. R. Angheluță); Constanţa County: Pădurea Băneasa, 17.IX.1964, leg. ŞN; Oltina, Canaraua Fetii, 26-27.IX.1978, leg. APG; Mangalia, Pădurea Hagieni, 28.IX.1978, leg. APG (Coll. Mus. G. ANTIPA).

Curculio (Curculio) venosus venosus (GRAVENHORST, 1807)

New data: Tulcea County: Podişul Babadagului, 17.V.2007, leg. NJ, RE, SoL, VJ; Măcin, Rezervaţia de la Greci, 23.IV.2009, leg. TLA; Slava Rusă, 2425.V.2013, leg. KI, PA, RI, SzL; Enisala, 29.IV3.V.2014, leg. KI, IZ, PA, SzL.

Curculio (Curculio) villosus FABRICIUS, 1781

Published data: TeOdor and Manole 1996, Raduta 2014

New data: Constanţa County: Esechioi (forest), 1-2. IV.2016, leg. FK, KI, PA, SzL.

Ellescini C. G. Thomson, 1859

Dorytomina BeDEL, 1886

Dorytomus (Dorytomus) filirostris (GYLLENHAL, 1835)
New data: Constanţa County: Pădurea Hagieni (forest), 6.VII.2014, leg. KI;

Dorytomus (Dorytomus) hirtipennis BEDEL, 1884 Published data: MonTandon 1908

New data: Tulcea County: Braţul Sfântu Gheorghe, 23.VI.2003, leg. TB; Smârdan, Braţul Măcin, 22.VI.2017, leg. KI, KJ, PA, TB; Greci (village), 22.VI.2017, leg. KI, KJ, PA, TB.

Dorytomus (Dorytomus) ictor (HERBST, 1795)

New data: Tulcea County: Braţul Sfântu Gheorghe, 21-22.VI.2003, leg. TB; Greci (village), 22.VI.2017,leg. KI, KJ, PA, TB; Nifon, pârâul Pârlita valley, 23.VI.2017, leg. TB; Codru Monastery, 24-25. VI.2017, leg. KI, KJ, PA, TB; Smârdan, Braţul Măcin, 2.VI.2018, leg. FK, KI, KJ, PA.

Dorytomus (Dorytomus) longimanus FORSTER, 1771 New data: Tulcea County: Slava Rusă, 24-25.V.2013, leg. KI, PA, RI, SzL; Constanţa County: Hagieni, 2.V.1993, leg. CP; Pădurea Hagieni, 22.V.1993, leg. CP, 22.V.1993, leg. CH (Coll. Mus. G. ANTIPA).

Dorytomus (Dorytomus) melanophthalmus (PAYKULL, 1792)

Published data: Montandon 1908

Dorytomus (Dorytomus) minutus (GYLLENHAL, 1835)

New data: Tulcea County: Greci (village), 22.VI.2017, leg. KI, KJ, PA, TB.

Dorytomus nebulosus (GYLLENHAL, 1835)

New data: Tulcea County: Smârdan, Braţul Măcin, 2.VI.2018, leg. FK, KI, KJ, PA.

\section{Dorytomus (Dorytomus) puberulus (BOHEMAN,} 1843)

New data: Constanţa County: Neptun-Olimp, Pădurea Comorova, 17.IX.2016, leg. FK, KI, KJ, PA.

Dorytomus (Dorytomus) suratus (GYLLENHAL, 1835) Published data: MonTANDON 1887, 1906; FleCK 1905

Dorytomus (Dorytomus) taeniatus (FABRICIUS, 1781) Published data: MonTANDON 1887, FleCK 1905

Dorytomus (Dorytomus) tremulae (FABRICIUS, 1787) Published data: MonTANDON 1887, FleCK 1905

Ellescina C. G. Thomson, 1859

Ellescus infirmus (HERBST, 1795)

Published data: MonTANDON 1887, FleCK 1905

Mecinini Gistel, 1848

Cleopomiarus graminis (GYLLENHAL, 1813)

Published data: Montandon 1887, FleCK 1905

New data: Constanţa County: Pădurea Hagieni, 
16.VII.1997, leg. AV; Cotu Văii, 23.VII.1997, leg. IoS, CP (Coll. Mus. G. AntIPA) - Cochirleni, 8.VII.2014, leg. KI, OA, PA, SzL; Gura Dobrogei, 25-27.VI.2017, leg. KI.

Gymnetron aper DesBrochers des Loges, 1892

New data: Tulcea County: Greci, 22.VI.1963, leg. IS (Coll. Mus. G. ANTIPA) - Codru Monastery, 2325.V.2013, leg. KI, PA, RI, SzL; Enisala, 29.IV3.V.2014, leg. KI, IZ, PA, SzL; Horea, 3.VI.2018, leg. KI; Constanţa County: $3 \mathrm{~km}$ W of Crucea, Allah Bair (Nature Reserve), 1.V.2009, leg. JK; Gura Dobrogei, 26.V.2013, leg. KI, PA, RI, SzL, 30.IV-2.V.2014, leg. KI, IZ, PA, SzL; Esechioi (forest), 7.VII.2014, leg. KI, OA, PA, SzL; $2 \mathrm{~km}$ from Sinoie to SE, 5.VI.2018, leg. FK, KI, KJ, PA.

\section{Gymnetron melanarium (GERMAR, 1821)}

New data: Tulcea County: Slava Rusă, 1-3.V.2014, leg. KI, IZ, PA, SzL; Babadag (forest), 13.IX.2016, leg. KI.

Gymnetron niloticum KIRSCH, 1881 (= furcatum DesBrochers des Loges, 1892)

Published data: (CAldara and Podlussány 2018)

New data: Tulcea County: Smârdan, Braţul Măcin, 7.V.2017, leg. FK, KI, KJ, PA, 22.VI.2017, leg. KI, KJ, PA, TB, 2.VI.2018, leg. KI; N from Nifon, 23.VI.2017, leg. KI, KJ, PA, TB; Constanţa County: $2 \mathrm{~km}$ from Sinoie to SE, 5.VI.2018, leg. FK, KI, KJ, PA.

\section{Gymnetron rostellum (HERBST, 1795)}

New data: Tulcea County: Codru Monastery, 30-31. III.2016, leg. FK, KI, PA, SzL; Constanţa County: Cochirleni, 3.IV.2016, leg. FK, KI, PA. SzL.

\section{Gymnetron rotundicolle GYLLENHAL, 1838}

New data: Tulcea County: M-ţii Măcin, Luncaviţa, 30.V.2005, leg. CsS; Codru env., 4-5.V.2009, leg. JK; Stejaru env., 5.V.2009, leg. JK; Atmagea, 28.IV.2012, leg. KI; Codru Monastery, 23-25.V.2013, leg. KI, PA, RI, SzL, 30-31.III.2016, leg. FK, KI, PA, SzL; Babadag (forest), 30.IV.2014, leg. KI, IZ, PA, SzL; Enisala, 29.IV-3.V.2014, leg. KI, IZ, PA, SzL; Slava Rusă, 1-3.V.2014, leg. KI, IZ, PA, SzL; Constanţa County: Gura Dobrogei, 26.V.2013, leg. KI, PA, RI, SzL, 12.V.2017, leg. KI; Murfatlar, Fântâniţa, 4-5. VII.2014, leg. KI, OA, PA, SzL, 22.V.2015, leg. PA, RI, SzK, SzL, 24-25.IX.2015, leg. KI, KJ, PA; Palazu Mic, 22.V.2015, leg. PA, RI, SzK, SzL; Băneasa, Canaraua Fetii, 23.V.2015, leg. PA, RI, SzK, SzL; Ostrov, 2.IV.2016, leg. FK, KI, PA. SzL; Cochirleni, 3.IV.2016, leg. FK, KI, PA, SzL.

Gymnetron stimulosum (GERMAR, 1821)

Published data: (CALdara and Podlussány 2018)

New data: Tulcea County: M-ţii Măcin, Valea Fagilor (quarry), 30.V.2005, leg. CsS; Codru Monastery,
30-31.III.2016, leg. FK, KI, PA, SzL; Greci (village), 8.V.2017, leg. FK, KI, KJ, PA; N from Nifon, 23.VI.2017, leg. KI, KJ, PA, TB; Constanţa County: Gura Dobrogei, 1.IV.2017, leg. FK, KI, PA. SzL.

*Gymnetron tibiellum Desbrochers des Loges, 1900

New data: Tulcea County: Smârdan, Braţul Măcin, 2.VI.2018, leg. FK, KI, KJ, PA; Constanţa County: $2 \mathrm{~km}$ from Sinoie to SE, 5.VI.2018, leg. FK, KI, KJ, PA; Sinoie, 21, 23.VI.2019, leg. KI, KJ, PA, TB.

\section{Gymnetron veronicae (GERMAR, 1821)}

New data: Tulcea County: M-ţii Măcin, Valea Fagilor (dried lake bed), 30.V.2005, leg. CsS; M-ţii Măcin, Valea Fagilor (quarry), 30.V.2005, leg. CsS; Smârdan, Braţul Măcin, 7.V.2017, leg. FK, KI, KJ, PA; S from Hamcearca, 24.VI.2017, leg. KI.

\section{Gymnetron villosulum GyLlenHAL, 1838}

New data: Tulcea County: Olguţa, 11.V.2007, leg. NJ, RE, SoL, VJ; Smârdan, Braţul Măcin, 7.V.2017, leg. FK, KI, KJ, PA, 2.VI.2018, leg. FK, KI, KJ, PA.

\section{Mecinus circulatus (MARSHAM, 1802)}

New data: Tulcea County: M-ţii Măcin, Valea Fagilor (dried lake bed), 30.V.2005, leg. CsS; Stejaru env., 5.V.2009, leg. JK; M-ţii Măcin, 5 km S Greci, 8.V.2017, leg. FK, KI, KJ, PA; Constanţa County: Pădurea Hagieni (forest), 6.VII.2014, leg. KI, OA, PA, SzL; Cheile Dobrogei, 2.V.2014, leg. KI, IZ, PA, SzL.

\section{Mecinus ictericus (GYLLENHAL, 1838)}

New data: Constanţa County: Istria env., Cetatea Histria, 2-4.V.2009, leg. JK, 20-22.IX.2010, leg. JK; Istria, Cetatea Histria, 14.IX.2016, leg. KI, KJ, PA, TB, 26-28.VI.2017, leg. KI, KJ, PA, TB, 6.VI.2018, leg. FK, KI, KJ, PA; Sinoie, Grindul Lupilor, 27.VI.2017, leg. KI, KJ, PA, TB, 5.VI.2018, leg. FK, KI, KJ, PA; Vadu, Grindul Chituc, 6.VI.2018, leg. FK, KI, KJ, PA.

Mecinus janthiniformis TOŠEVSKI \& CALDARA, 2011 New data: Tulcea County: C. A. Rosetti, 8.VI.1981, leg. CP (Coll. Mus. G. AntiPA) - Codru Monastery 23-25.V.2013, leg. KI, PA, RI, SzL, 10-11.V.2017, leg. FK, KI, KJ, PA, 4.VI.2018, leg. FK, KI, KJ, PA; Slava Rusă, 24-25.V.2013, leg. KI, PA, RI, SzL; Enisala (wooded pasture), 4.VI.2018, leg. FK, KI, KJ, PA; Constanţa County: Histria, 21.V.2015, leg. PA, RI, SzK, SzL.

\section{Mecinus janthinus GERMAR, 1821}

New data: Tulcea County: Codru Monastery, 10 11.V.2017, leg. FK, KI, KJ, PA, 4.VI.2018, leg. FK, KI, KJ, PA; Enisala (wooded pasture), 4.VI.2018, leg. FK, KI, KJ, PA; Constanţa County: $3 \mathrm{~km} \mathrm{~W}$ of Crucea, Allah Bair (Nature Reserve), 1.V.2009, leg. JK; Cochirleni, 24.V.2015, leg. PA, RI, SzK, SzL, 
3.IV.2016, leg. FK, KI, PA, SzL; Vadu, Grindul Chituc, 6.VI.2018, leg. FK, KI, KJ, PA; Murfatlar, Fântâniţa, 7-8.VI.2018, leg. FK, KI, KJ, PA.

\section{Mecinus labilis (HERBST, 1795)}

New data: Tulcea County: M-ţii Măcin, Valea Fagilor (quarry), 30.V.2005, leg. CsS; M-ţii Măcin, to the Peak Ţuţuiatu, 28.IV.2010, leg. TLA; Atmagea, 28.IV.2012, leg. KI; Codru Monastery 23-25.V.2013, leg. KI, PA, RI, SzL, 10-11.V.2017, leg. FK, KI, KJ, PA; Enisala, 24-25.V.2013, leg. KI, PA, RI, SzL, 29.IV-3.V.2014, leg. KI, IZ, PA, SzL; M-ţii Măcin, Greci, 7.V.2017, leg. FK, KI, KJ, PA; M-ţii Măcin, 5 km S Greci, 8.V.2017, leg. FK, KI, KJ, PA; Smârdan, Braţul Măcin, 7.V.2017, leg. FK, KI, KJ, PA; Slava Rusă, 11.V.2017, leg. FK, KI, KJ, PA; Enisala (castle), 20.VI.2019, leg. KI, KJ, PA, TB; Constanţa County: Furnica, Rezervaţia Dumbrăveni, 13-14.V.1995, leg. CP; Negureni, 21.VI.1996, leg. CP (Coll. Mus. G. Antipa) - Gura Dobrogei, 28-29. IV.2012, leg. KI; Murfatlar, Fântâniţa, 4-5.VII.2014, leg. KI, OA, PA, SzL, 22.V.2015, leg. PA, RI, SzK, SzL; Esechioi (forest), 7.VII.2014, leg. KI, OA, PA, SzL; Palazu Mic, 22.V.2015, leg. PA, RI, SzK, SzL; Hagieni, Pădurea Hagieni (forest), 17.IX.2016, leg. FK, KI, KJ, PA; Limanu, 12.V.2017, leg. FK, KI, KJ, PA; Albeşti, Pădurea Hagieni (forest), 12.V.2017, leg. KI; Istria, Cetatea Histria, 6.VI.2018, leg. FK, KI, KJ, PA.

\section{Mecinus pascuorum (GYLLENHAL, 1813)}

Published data: MontANDON 1887, FleCK 1905, Negru and Roşca 1967, TeOdor and Manole 1996, RADUTA 2014

New data: Tulcea County: M-ţii Măcin, Valea Fagilor (quarry), 30.V.2005, leg. CsS; Frecăţei, Celic Dere Monastery (hillside), 31.V.2005, leg. CsS; Babadag (forest), 23-25.V.2013, leg. KI, PA, RI, SzL; Codru Monastery, 23-25.V.2013, leg. KI, PA, RI, SzL, 24 25.VI.2017, leg. KI, KJ, PA, TB, 4.VI.2018, leg. FK, KI, KJ, PA, 20.VI.2019, leg. KI, KJ, PA, TB; M-ţii Măcin, 5 km S Greci, 8.V.2017, leg. FK, KI, KJ, PA; Turcoaia, $6 \mathrm{~km}$ from Cerna to NW, 24.VI.2017, leg. KI, KJ, PA, TB, 3.VI.2018, leg. FK, KI, KJ, PA, 19.VI.2019, leg. KI, KJ, PA, TB; Enisala, Cetatea Heraclea, 25.VI.2017, leg. KI, KJ, PA, TB; Horea, 3.VI.2018, leg. FK, KI, KJ, PA; General Praporgescu, 3.VI.2018, leg. FK, KI, KJ, PA; 2 km from Slava Rusă to NE, 3.VI.2018, leg. FK, KI, KJ, PA; M-ţii Măcin, Greci, 18-19.VI.2019, leg. KI, KJ, PA, TB; Enisala (castle), 20.VI.2019, leg. KI, KJ, PA, TB; Enisala (forest), 20.VI.2019, leg. KI, KJ, PA, TB; Constanţa County: Istria env., Cetatea Histria, 2-4.V.2009, leg. JK, 26-28.VI.2017, leg. KI, KJ, PA, TB, 6.VI.2018, leg. FK, KI, KJ, PA; Esechioi (forest), 7.VII.2014, leg. KI, OA, PA, SzL; Palazu Mic, 22.V.2015, leg. PA, RI, SzK, SzL; Băneasa, Canaraua Fetii, 23.V.2015, leg. PA, RI, SzK, SzL; $2 \mathrm{~km}$ from Sinoie to SE, 27.VI.2017, leg. KI, KJ, PA, TB, 5.VI.2018, leg. FK, KI, KJ, PA;
Sinoie, Grindul Lupilor, 27.VI.2017, leg. KI, KJ, PA, TB, 5.VI.2018, leg. FK, KI, KJ, PA, 22.VI.2019, leg. KI, KJ, PA, TB; Vadu, Grindul Chituc, 6.VI.2018, leg. FK, KI, KJ. PA; Crucea, 9.VI.2018, leg. PA.

\section{Mecinus pirazzolii (STIERLIN, 1867)}

Published data: Montandon 1908, Negru 1968, Teodor and Manole 1996, Raduta 2014, Caldara and PodLussánY 2018

New data: Tulcea County: M-ţii Măcin, Valea Fagilor (quarry), 30.V.2005, leg. CsS; Smârdan, Braţul Măcin, 2.VI.2018, leg. FK, KI, KJ, PA; Jurilovca, 23.VI.2019, leg. KI, KJ, PA, TB; Constanţa County: Istria env., Cetatea Histria, 20-22.IX.2010, leg. JK, 14.IX.2016, leg. KI, KJ, PA, TB, 11.V.2017, leg. FK, KI, KJ, PA, 26-28.VI.2017, leg. KI, KJ, PA, TB, 6.VI.2018, leg. FK, KI, KJ, PA, 21.VI.2019, leg. KI, KJ, PA, TB; Gura Dobrogei, 25-27.VI.2017, leg. KI, KJ, PA, TB; Sinoie, Grindul Lupilor, 27.VI.2017, leg. KI, KJ, PA, TB, 5.VI.2018, leg. FK, KI, KJ, PA; Vadu, Grindul Chituc, 6.VI.2018, leg. FK, KI, KJ, PA, 22.VI.2019, leg. KI, KJ, PA, TB.

\section{Mecinus pyraster (HERBST, 1795)}

Published data: Montandon 1887, Fleck 1905, Negru and Roşca 1967, Raduta 2014

New data: Tulcea County: M-ţii Măcin, Valea Fagilor (quarry), 30.V.2005, leg. CsS; Enisala, 24-25.V.2013, leg. KI, PA, RI, SzL; M-ţii Măcin, Greci, 8.V.2017, leg. FK, KI, KJ, PA; M-ţii Măcin, 5 km S Greci, 8.V.2017, leg. FK, KI, KJ, PA; Codru Monastery, 10-11.V.2017, leg. FK, KI, KJ, PA; Greci (village), 8.V.2017, leg. FK, KI, KJ, PA; Constanţa County: Pădurea Comorova, 3.VIII.1992, leg. AS (Coll. Mus. G. ANTIPA) - Albeşti, Pădurea Hagieni (forest), 12.V.2017, leg. FK, KI, KJ, PA; Murfatlar, Fântâniţa, 7-8.VI.2018, leg. KI.

Mecinus simus (MulsANT \& ReY, 1859)

Published data: Montandon 1887, Fleck 1905, Negru and RoşCa 1967, Raduta 2014

Miarus campanulae (LINNÉ, 1767)

Published data: Fleck 1905, Montandon 1908

New data: Constanţa County: Hagieni, 17-22. VII.1997, leg. CP, (Coll. Mus. G. ANTIPA) - Gura Dobrogei, 7.VI.2018, leg. FK, KI, KJ, PA.

\section{*Miarus hellenicus Dieckmann, 1978}

New data: Constanţa County: Cotu Văii, 23.VII.1997, leg. CP (Coll. Mus. G. AnTIPA).

\section{*Miarus solarii SMRECZYŃSKI, 1957}

New data: Constanţa County: Cochirleni, 8.VII.2014, leg. KI, OA, PA, SzL.

\section{Rhinusa antirrhini (PAYKULL, 1800)}

Published data: Montandon 1908, Peruu and PăLĂgeşiu 1986, TeOdor and Manole 1996, Raduta 
2014

New data: Tulcea County: Enisala, Cetatea Heraclea, 25.VI.2017, leg. KI, KJ, PA, TB; Babadag (forest), 24-26.VI.2017, leg. KI, KJ, PA, TB; Gura Dobrogei, 25-27.VI.2017, leg. KI; Enisala (wooded pasture), 4.VI.2018, leg. FK, KI, KJ, PA; Jurilovca, 23.VI.2019, leg. KI, KJ, PA, TB; Constanţa County: Sinoie, Grindul Lupilor, 27.VI.2017, leg. KI; Vadu, Grindul Chituc, 6.VI.2018, leg. FK, KI, KJ, PA, 22.VI.2019, leg. KI, KJ, PA, TB.

\section{Rhinusa asellus (GRAVENHORST, 1807)}

Published data: Teodor and Manole 1996, Raduta 2014

New data: Tulcea County: M-ţii Măcin, Pricopan (300-370 m), 16.VI.2005, leg. GP (Coll. Mus. R. ANGHELUȚĂ); Luncaviţa, Parcul Naţional M-ţii Măcin, 24.V.2005, leg. MS; Periprava, Păd. Letea, 23.VI.1966, leg. ŞN(Coll.Mus. G.ANTIPA) -Caraorman, 14.V.1992, leg. TLA; Iancina, 11.VI.1993, leg. TLA; Pădurea Letea (forest), 17.VI.1993, leg. TLA; M-ţii Măcin, to the Peak Ţuţuiatu, 28.IV.2010, leg. TLA; Enisala, 2425.V.2013, leg. KI, PA, RI, SzL, 29.IV-3.V.2014, leg. KI, IZ, PA, SzL; Slava Rusă, 24-25.V.2013, leg. KI, PA, RI, SzL; M-ţii Măcin, Greci,8.V.2017, leg. FK, KI, KJ, PA, 18-19.VI.2019, leg. KI, KJ, PA, TB; M-ţii Măcin, 5 km S Greci, 8.V.2017, leg. FK, KI, KJ, PA; Codru Monastery, 10-11.V.2017, leg. FK, KI, KJ, PA; Nifon, pârâul Pârlita valley, 23.VI.2017, leg. KI, KJ, PA, TB; N from Nifon, 23.VI.2017, leg. KI, KJ, PA, TB; S from Hamcearca, 24.VI.2017, leg. KI, KJ, PA, TB; Smârdan, Braţul Măcin, 28.VI.2017, leg. KI, KJ, PA, TB; $3 \mathrm{~km}$ from Slava Rusă to NE, 24.VI.2017, leg. KI, KJ, PA, TB; 2 km from Slava Rusă to NE, 3.VI.2018, leg. FK, KI, KJ, PA; Constanţa County: Hagieni, 4.VI.1964, leg. NS; Târguşor, 8.VII.1992, leg. RS; Furnica, 22.VI.1995, leg. CP (Coll. Mus. G. ANTIPA) - Gura Dobrogei, 28-29.IV.2012, leg. KI; Vadu, Grindul Chituc, 22.VI.2019, leg. KI.

Rhinusa bipustulata (Rossi, 1792)

New data: Tulcea County: Luncaviţa, Valea Fagilor, 23.V.1997, leg. RS (Coll. Mus. G. Antipa); Constanța County: Hagieni, 22.V.1993, leg. CH (Coll. Mus. G. ANTIPA).

Rhinusa collina (GYLLENHAL, 1813)

New data: Tulcea County: Turcoaia, $6 \mathrm{~km}$ from Cerna to NW, 24.VI.2017, leg. KI, KJ, PA, TB.

Rhinusa florum (RÜBSAAMEN, 1895) (= Gymnetron smreczynskii FrEMUTH, 1972)

Published data: Caldara and Podlussány 2018

New data: Tulcea County: Codru Monastery, 30 31.III.2016, leg. FK, KI, PA, SzL; Enisala, Cetatea Heraclea, 25.VI.2017, leg. KI, KJ, PA, TB; Enisala (wooded pasture), 4.VI.2018, leg. FK, KI, KJ, PA; Jurilovca, 23.VI.2019, leg. KI; Constanţa County: Sinoie, Grindul Lupilor, 27.VI.2017, leg. KI, KJ,
PA, TB, 5.VI.2018, leg. FK, KI, KJ, PA, 22.VI.2019, leg. KI, KJ, PA, TB; Sinoie, 21, 23.VI.2019, leg. KI; Vadu, Grindul Chituc, 6.VI.2018, leg. FK, KI, KJ, PA, 22.VI.2019, leg. KI, KJ, PA, TB.

\section{Rhinusa linariae (PANZER, 1795)}

New data: Tulcea County: Codru Monastery, 10.V.2017, leg. KI; Constanţa County: Furnica, Rezervaţia Dumbrăveni, 13-14.V.1995, leg. CP (Coll. Mus. G. ANTIPA) $-3 \mathrm{~km}$ W of Crucea, Allah Bair (Nature Reserve), 1.V.2009, leg. JK; Cochirleni, 3.IV.2016, leg. FK, KI, PA, SzL.

\section{Rhinusa neta (GERMAR, 1821)}

Published data: HurmuZaCHI 1904

New data: Tulcea County: M-ţii Măcin, Valea Fagilor (quarry), 30.V.2005, leg. CsS; M-ţii Măcin, Luncaviţa, 30.V.2005, leg. CsS; M-ţii Măcin, to the Peak Ţuţuiatu, 28.IV.2010, leg. TLA; Codru Monastery, 10-11.V.2017, leg. FK, KI, KJ, PA, 4.VI.2018, leg. $\mathrm{KI} ; 1,5 \mathrm{~km}$ from Beidaud to SW, 25.VI.2017, leg. KI; Enisala (wooded pasture), 4.VI.2018, leg. FK, KI, KJ, PA; Smârdan, Braţul Măcin, 18.VI.2019, leg. KI; Constanţa County: $3 \mathrm{~km} \mathrm{~W}$ of Crucea, Allah Bair (Nature Reserve), 18.IX.2010, leg. JK; Cheia env., Cheia Nature Reserve., 19.IX.2010, leg. JK; Balta Limanu, 5-6.VII.2014, leg. KI, OA, PA, SzL; Sinoie, Grindul Lupilor, 27.VI.2017, leg. KI, 5.VI.2018, leg. FK, KI, KJ, PA; Vadu, Grindul Chituc, 6.VI.2018, leg. FK, KI, KJ, PA; Sinoie, 21, 23.VI.2019, leg. KI.

\section{Rhinusa tetra (FABRICIUS, 1792)}

Published data: Montandon 1887; HurmuzachI 1904; FelCK 1905; Negru 1957, 1968; Negru and RoşCa 1967; PerJu and PăLĂGEşıU 1986; TeOdor and Manole 1996; Raduta 2014

New data: Tulcea County: M-ţii Măcin, Pricopan (300-370 m), 16.VI.2005, leg. GP (Coll. Mus. R. ANGHELUȚǍ); Cerna, 26.V.2005, leg. IM; Măcin, Valea Suluc, 27.V.2005, leg. CB, (Coll. Mus. G. ANTIPA) - Măcin (hillside, 200 m), 29.V.2005, leg. CsS; M-ţii Măcin, Valea Fagilor (quarry), 30.V.2005, leg. CsS; Slava Rusă, 24-25.V.2013, leg. KI, PA, RI, SzL, 20.V.2015, leg. PA, RI, SzK, SzL, 9.V.2017, leg. FK, KI, KJ, PA; Babadag (forest), 23-25.V.2013, leg. KI, PA, RI, SzL; Codru Monastery, 23-25.V.2013, leg. KI, PA, RI, SzL, 30.IV-3.V.2014, leg. KI, IZ, PA, SzL, 24-25.VI.2017, leg. KI, KJ, PA, TB, 4.VI.2018, leg. KI, 20.VI.2019, leg. KI, KJ, PA, TB; M-ţii Măcin, Greci, 8.V.2017, leg. FK, KI, KJ, PA, 18-19.VI.2019, leg. KI, KJ, PA, TB; M-ţii Măcin, 5 km S Greci, 8.V.2017, leg. FK, KI, KJ, PA; Nifon, pârâul Pârlita valley, 23.VI.2017, leg. KI, KJ, PA, TB; N from Nifon, 23.VI.2017, leg. KI, KJ, PA, TB; Turcoaia, 6 $\mathrm{km}$ from Cerna to NW, 24.VI.2017, leg. KI, KJ, PA, TB; S from Hamcearca, 24.VI.2017, leg. KI, KJ, PA, TB; Jijila, râul Jijila, 23.VI.2017, leg. KI, KJ, PA, TB; Smârdan, Braţul Măcin, 22.VI.2017, leg. KI, KJ, PA, TB; 3 km from Slava Rusă to NE, 24.VI.2017, 
leg. KI, KJ, PA, TB; 2 km from Slava Rusă to NE, 3.VI.2018, leg. FK, KI, KJ, PA; Enisala (wooded pasture), 4.VI.2018, leg. FK, KI, KJ PA; Enisala (castle), 20.VI.2019, leg. KI, KJ, PA, TB; Enisala (forest), 20.VI.2019, leg. KI, KJ, PA, TB; Sinoie, 21, 23.VI.2019, leg. KI, KJ, PA, TB; Constanţa County: Gura Dobrogei, 28-29.IV.2012, leg. KI, 12.V.2017, leg. FK, KI, KJ, PA, 7.VI.2018, leg. FK, KI, KJ, PA; Murfatlar, Fântâniţa, 4-5.VII.2014, leg. KI, OA, PA, SzL, 24-25.IX.2015, leg. KI, KJ, PA; Balta Limanu, 5-6.VII.2014, leg. KI, OA, PA, SzL; Esechioi (forest), 7.VII.2014, leg. KI, OA, PA, SzL, 1-2.IV.2016, leg. FK, KI, PA. SzL; Cochirleni, 8.VII.2014, leg. KI, OA, PA, SzL; Istria, Cetatea Histria, 21.V.2015, leg. PA, RI, SzK, SzL, 26-28.VI.2017, leg. KI, KJ, PA, TB; Sinoie, Grindul Lupilor, 27.VI.2017, leg. KI, KJ, PA, TB; Vadu, Grindul Chituc, 22.VI.2019, leg. KI, KJ, PA, TB.

Rhamphini RAFINESQUE, 1815

Rhamphina RAFINESQUE, 1815

Isochnus sequensi (STIERLIN, 1894) (= populi FABRICIUS, 1792; = populicola SILFVERBERG, 1977)

New data: Tulcea County: C. A. Rosetti, VII.1950 (Coll. Mus. G. ANTIPA) - Slava Rusă, 9.V.2017, leg. FK, KI, KJ, PA.

Orchestes (Alyctus) testaceus (O. F. MÜLLER, 1776) New data: Tulcea County: Periprava, Deltă, 08.V.1964, leg. MW; Periprava, Păd. Letea, 23.VI.1966, leg. ŞN (Coll. Mus. G. Antipa) - Greci (village), 22.VI.2017, leg. KI.

Orchestes (Orchestes) betuleti (PANZER, 1795)

(= rufus SCHRANK, 1781)

Published data: (FLECK 1905)

Orchestes (Orchestes) erythropus (GERMAR, 1821) (=Orchestes erythropus tricolor KIESENWETTER, 1852) Published data: MONTANDON 1908

Orchestes (Orchestes) hortorum FABRICIUS, 1792 (= avellanae Donovan, 1797; signifer (CREUTZER, 1799) New data: Tulcea County: C. A. Rosetti, Păd. Letea, 11.V.1964, leg. XS (Coll. Mus. G. ANTIPA).

Orchestes (Orchestes) hungaricus (HAJÓss, 1938) Published data: Teodor and Manole 1996

Orchestes (Orchestes) pilosus (FABRICIUS, 1781)

Published data: MONTANDON 1908

New data: Tulcea County: Cerna, 26.V.2005, leg. CB (Coll. Mus. G. ANTIPA) - Babadag, (forest), 23 25.V.2013, leg. KI, PA, RI, SzL; $3 \mathrm{~km}$ from Slava Rusă to NE, 24.VI.2017, leg. KI; M-ţii Măcin, 5 km S Greci, 8.V.2017, leg. KI; Constanţa County: Băneasa, Canaraua Fetii (quarry), 2-3.IV.2016, leg. FK, KI, PA, SzL.
Orchestes (Orchestes) quercus (LINNÉ, 1758)

Published data: IENIŞTEA 1974

New data: Constanţa County: Esechioi (forest), 1-2. IV.2016, leg. FK, KI, PA, SzL.

\section{Orchestes (Orchestes) subfasciatus GYLLENHAL,} 1835

Published data: DRĂGHIA 1972

Pseudorchestes cinereus (FÅHAEUS, 1843)

Published data: JAQUET 1899b, FLECK 1905, NEGRU and RoşCa 1967, RADUTA 2014

Pseudorchestes michalki (DieCKMANN, 1963)

Published data: DieCKMANn 1963

Pseudorchestes pratensis (GERMAR, 1821)

Published data: Negru and RoşCa 1967; Raduta 2014

New data: Tulcea County: Smârdan, Braţul Măcin, 28.VI.2017, leg. KI, KJ, PA, TB.

Pseudorchestes smreczynskii (DIECKMANN, 1958)

New data: Constanţa County: Esechioi (forest), 7.VII.2014, leg. KI, OA, PA, SzL.

Rhamphus oxyacanthae (MARSHAM, 1802)

New data: Tulcea County: Slava Rusă, 24-25.V.2013, leg. KI, PA, RI, SzL, 11.V.2017, leg. FK, KI, KJ, PA.

Rhamphus pulicarius (HERBST, 1795)

Published data: FLECK 1905, Negru and RoşCA 1967

Rhamphus subaeneus (ILLIGER, 1807)

New data: Constanţa County: Băneasa, Canaraua Fetii, 23.V.2015, leg. PA, RI, SzK, SzL.

\section{Tachyerges salicis (LINNÉ 1758)}

Published data: DRĂGHIA 1972

New data: Tulcea County: Tulcea, 26.VII.1959, leg. GL (Coll. Hung. Nat. Hist. Mus.) - M-ţii Măcin, 5 km S Greci, 8.V.2017, leg. FK, KI, KJ, PA; Smârdan, Brațul Măcin, 22.VI.2017, leg. KI, KJ, PA, TB, 2.VI.2018, leg. FK, KI, KJ, PA.

Smicronychini SeIDLITZ, 1891

Sharpia soluta FAUST, 1885

Published data: Zumpt 1935, Osella and Di Marco 1993

New data: Tulcea County: M-ţii Măcin, Dealurile Pricopanului, 14.VI.2007, leg. CŞ (Coll. Mus. R. ANGHELuȚă) - Măcin (hillside, 200 m), 29.V.2005, leg. CsS; Greci (village), 8.V.2017, leg. FK, KI, KJ, PA; Codru Monastery, 24-25.VI.2017, leg. KI, KJ, PA, TB, 20.VI.2019, leg. KI, KJ, PA, TB; Enisala (castle), 20.VI.2019, leg. KI, KJ, PA, TB; Jurilovca, 23.VI.2019, leg. KI, KJ, PA, TB; Constanţa County: Hagieni, 7.VI.1965, leg. ŞN (Coll. Mus. G. ANTIPA) - Gura Dobrogei, 26.V.2013, leg. KI, PA, RI, SzL; 
Esechioi (forest), 7.VII.2014, leg. KI, OA, PA, SzL; Balta Limanu, 5-6.VII.2014, leg. KI, OA, PA, SzL.

\section{Smicronyx coecus (REICH, 1797)}

New data: Tulcea County: Nifon, pârâul Pârlita valley, 23.VI.2017, leg. KI, KJ, PA, TB; Codru Monastery, 24-25.VI.2017, leg. KI, KJ, PA, TB, 20.VI.2019, leg. KI, KJ, PA, TB; Constanţa County: Limanu, 12.V.2017, leg. FK, KI, KJ, PA; Gura Dobrogei, $25-$ 27.VI.2017, leg. KI, KJ, PA, TB; Murfatlar, Fântâniţa, 7-8.VI.2018, leg. FK, KI, KJ, PA.

\section{Smicronyx jungermanniae (REICH, 1797)}

Published data: Montandon 1887, Fleck 1905, TEOdOR and Manole 1996, Raduta 2014

New data: Tulcea County: M-ţii Măcin, to the Peak Ţuţuiatu, 28.IV.2010, leg. TLA; Codru Monastery, 2325.V.2013, leg. KI, PA, RI, SzL, 19.V.2015, leg. PA, RI, SzK, SzL; Enisala (castle), 24-25.V.2013, leg. KI, PA, RI, SzL, 22.IX.2015, leg. KI, KJ, PA, 10.V.2017, leg. FK, KI, KJ, PA; Smârdan, Braţul Măcin, 21-23. IX.2015,leg. KI, KJ, PA, 7.V.2017, leg. FK, KI, KJ, PA, 22.VI.2017, leg. KI, KJ, PA, TB, 2.VI.2018, leg. FK, KI, KJ, PA; Sălcioara, Lacul Sălcioara, 13.IX.2016, leg. KI, KJ, PA, TB; M-ţii Măcin, Greci, 8.V.2017, leg. FK, KI, KJ, PA, 18-19.VI.2019, leg. KI, KJ, PA, TB; Turcoaia, $6 \mathrm{~km}$ from Cerna to NW, 24.VI.2017, leg. KI; Constanţa County: Furnica, Rezervaţia Dumbrăveni, 13-14.V.1995, leg. CP (Coll. Mus. G. ANTIPA) - Grădina env., 19.IX.2010, leg. JK; Gura Dobrogei, 28-29.IV.2012, leg. KI, 26.V.2013, leg. KI, PA, RI, SzL, 25-27.VI.2017, leg. KI, KJ, PA, TB; Murfatlar, Fântâniţa, 4-5.VII.2014, leg. KI, OA, PA, SzL, 22.V.2015, leg. PA, RI, SzK, SzL; Esechioi (forest), 1-2.IV.2016, leg. FK, KI, PA, SzL; Bugeac, 2.IV.2016, leg. FK, KI, PA, SzL; Pecineaga (forest), 14.V.2017, leg. FK, KI, KJ, PA; Hagieni, Pădurea Hagieni (forest), 17.IX.2016, leg. FK, KI, KJ, PA; Albeşti, Pădurea Hagieni (forest), 12.V.2017, leg. KI.

\section{Smicronyx reichii (GYLLENHAL, 1835)}

New data: Tulcea County: Enisala, 24-25.V.2013, leg. KI, PA, RI, SzL, 20.VI.2019, leg. KI; Constanţa County: Cheile Dobrogei, 2.V.2014, leg. KI, IZ, PA, SzL; Esechioi (forest), 7.VII.2014, leg. KI, OA, PA, SzL; Istria, Cetatea Histria, 14.IX.2016, leg. KI, KJ, PA, TB; Gura Dobrogei, 27.VI.2017, leg. KI, KJ, PA, TB.

\section{Smicronyx smreczynskii F. SOLARI, 1952}

New data: Tulcea County: M-ţii Măcin, Pricopan, 1.VI.2005, leg. CsS; Constanţa County: Gura Dobrogei, 26.V.2013, leg. KI, PA, RI, SzL; Esechioi (forest), 7.VII.2014, leg. KI, OA, PA, SzL.

\section{Smicronyx (Smicronyx) swertiae Voss, 1953}

New data: Tulcea County: C. A. Rosetti, 10.V.1964, leg. MW (Coll. Mus. G. ANTIPA) - Smârdan, Braţul Măcin, 28.VI.2017, leg. KI, KJ, PA, TB; Enisala, 24-
25.V.2013, leg. KI, PA, RI, SzL; Codru Monastery, 24-25.VI.2017, leg. KI, 20.VI.2019, leg. KI, KJ, PA, TB; Constanţa County: Gura Dobrogei, 27.VI.2017, leg. KI, KJ, PA, TB, 7.VI.2018, leg. FK, KI, KJ, PA; $2 \mathrm{~km}$ from Sinoie to SE, 27.VI.2017, leg. KI, KJ, PA, TB; Sinoie, Grindul Lupilor, 5.VI.2018, leg. KI; Istria, Cetatea Histria, 6.VI.2018, leg. FK, KI, KJ, PA; Vadu, Grindul Chituc, 22.VI.2019, leg. KI, KJ, PA, TB.

Smicronyx syriacus FAUST, 1887 (= brevicornis F. SOlARI, 1952)

New data: Tulcea County: Slava Rusă, 1-3.V.2014, leg. KI, IZ, PA, SzL.

\section{Styphlini JeKel, 1861}

Paraphilernus bilunulatus (DESBROCHERS des Loges, 1870)

Published data: Montandon 1908

New data: Constanţa County: Istria env., Cetatea Histria, 22.IX.2010, leg. JK; $2 \mathrm{~km}$ from Sinoie to SE, 5.VI.2018, leg. FK, KI, KJ, PA; 2 km from Slava Rusă to NE, 3.VI.2018, leg. KI.

\section{*Philernus ponticus Korotyaev, 1979 (fig. 15)}

New data: Constanţa County: $2 \mathrm{~km}$ from Sinoie to SE, 27.VI.2017, leg. KI, 21, 23.VI.2019, leg. KI, KJ, PA, TB.

\section{Pseudostyphlus pillumus (Gyllenhal, 1835)}

New data: Tulcea County: M-ţii Măcin, Valea Fagilor (quarry), 30.V.2005, leg. CsS; $1,5 \mathrm{~km}$ from Beidaud to SW, 25.VI.2017, leg. KI, KJ, PA, TB; Constanţa County: Hagieni, Pădurea Hagieni (forest), 17.IX.2016, leg. FK, KI, KJ, PA.

Tychiini C. G. Thomson, 1859

Lignyodina BEDEL, 1884

Lignyodes enucleator (PANZER, 1798)

New data: Tulcea County: Tulcea, 16.V.2007, leg. NJ, RE, SoL, VJ; Atmagea, 28.IV.2012, leg. KI; Babadag (forest), 30.IV.2014, leg. KI, IZ, PA, SzL, 10.V.2017, leg. KI; Constanţa County: Oltina, Pădurea Râzoarele (forest), 7.VII.2014, , leg. KI, OA, PA, SzL.

Tychiina C. G. Thomson, 1859

Sibinia (Dichotychius) beckeri (DESBROCHERS des LOGES, 1873)

New data: Constanţa County: Vadu env., Grindul Chituc, 21.IX.2010, leg. JK.

\section{Sibinia (Dichotychius) simulans CALDARA \&} KARASYOV, 1995

Published data: Caldara and Podlussány 2018

New data: Constanţa County: Cetatea Histria, pr. Năvodari, 18.VII.2009, leg. MK; $2 \mathrm{~km}$ from Sinoie to SE, 27.VI.2017, leg. KI, KJ, PA, TB, 5.VI.2018, leg. FK, KI, KJ, PA; Sinoie, Grindul Lupilor, 27.VI.2017, leg. KI, KJ, PA, TB. 


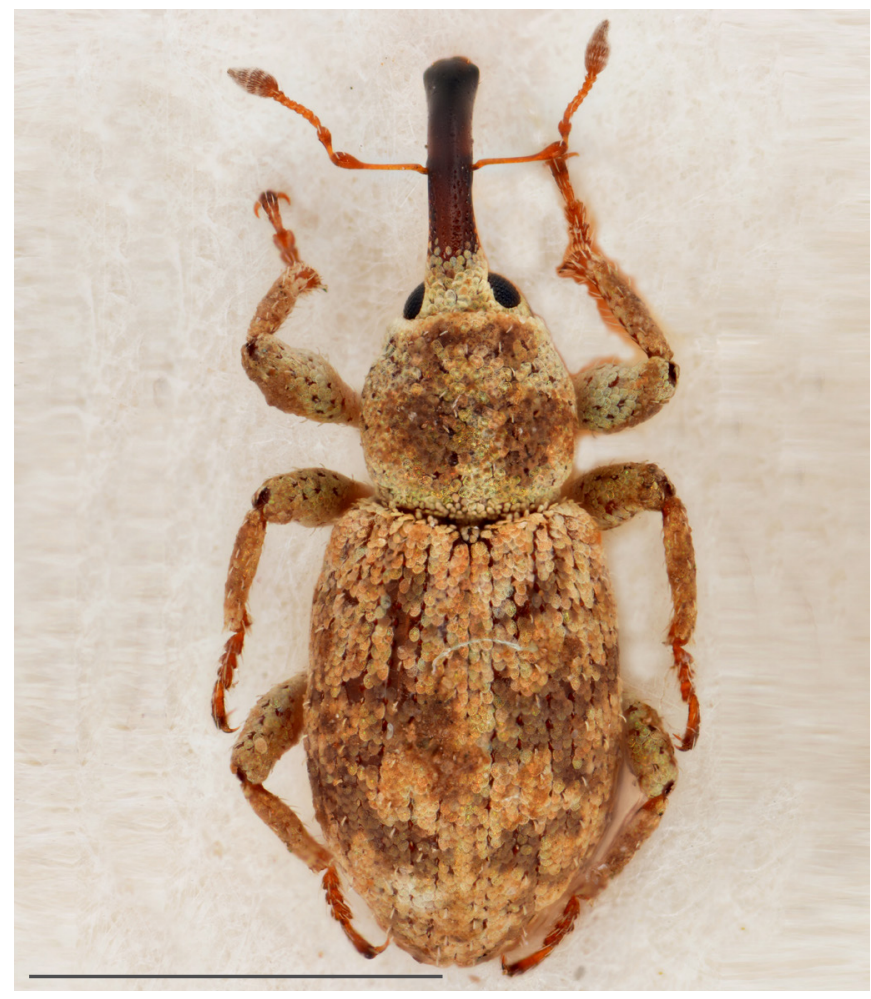

Fig. 15. Philernus ponticus KoRotyaev, 1979 . Scale bar: 1 $\mathrm{mm}$. Photo Tamás NÉMETH

Sibinia (Dichotychius) sodalis GERMAR, 1823 Published data: TeOdor and Manole 1996, Raduta 2014

New data: Constanţa County: Sinoie, Grindul Lupilor, 27.VI.2017, leg. KI, KJ, PA, TB; Gura Dobrogei, 25-27.VI.2017, leg. KI; Istria, Cetatea Histria, 6.VI.2018, leg. FK, KI, KJ, PA; $2 \mathrm{~km}$ from Sinoie to SE, 5.VI.2018, leg. KI.

\section{Sibinia (Sibinia) attalica GYLLENHAL, 1835}

New data: Tulcea County: C. A. Rosetti, Deltă, 12.V.1964, leg. MW; Măcin, Valea Suluc, 27.V.2005, leg. IM (Coll. Mus. G. Antipa) - M-ţii Măcin, Valea Fagilor (quarry), 30.V.2005, leg. CsS; Constanţa County: Gura Dobrogei, 12.V.2017, leg. FK, KI, KJ, PA; Sinoie, Grindul Lupilor, 27.VI.2017, leg. KI.

*Sibinia (Sibinia) femoralis GERMAR, 1823 (fig. 16) New data: Tulcea County: Pădurea Letea (forest), 17.VI.1993, leg. TLA; M-ţii Măcin, Greci, 7.V.2017, leg. FK, KI, KJ, PA; Constanţa County: Istria, Cetatea Histria, 11.V.2017, leg. FK, KI, KJ, PA; Sinoie, Grindul Lupilor, 27.VI.2017, leg. KI, KJ, PA, TB, 5.VI.2018, leg. FK, KI, KJ, PA; Vadu, Grindul Chituc, 6.VI.2018, leg. FK, KI, KJ, PA, 22.VI.2019, leg. KI, KJ, PA, TB.

Sibinia (Sibinia) pellucens (SCOPOLI, 1772)

New data: Tulcea County: Nifon, pârâul Pârlita valley, 23.VI.2017, leg. KI, KJ, PA, TB; S from Hamcearca, 24.VI.2017, leg. KI, KJ, PA, TB; Constanța County: Mangalia, Pădurea Comorova, 30.V.1963, leg. XS (Coll. Mus. G. ANTIPA) - Băneasa, Canaraua Fetii

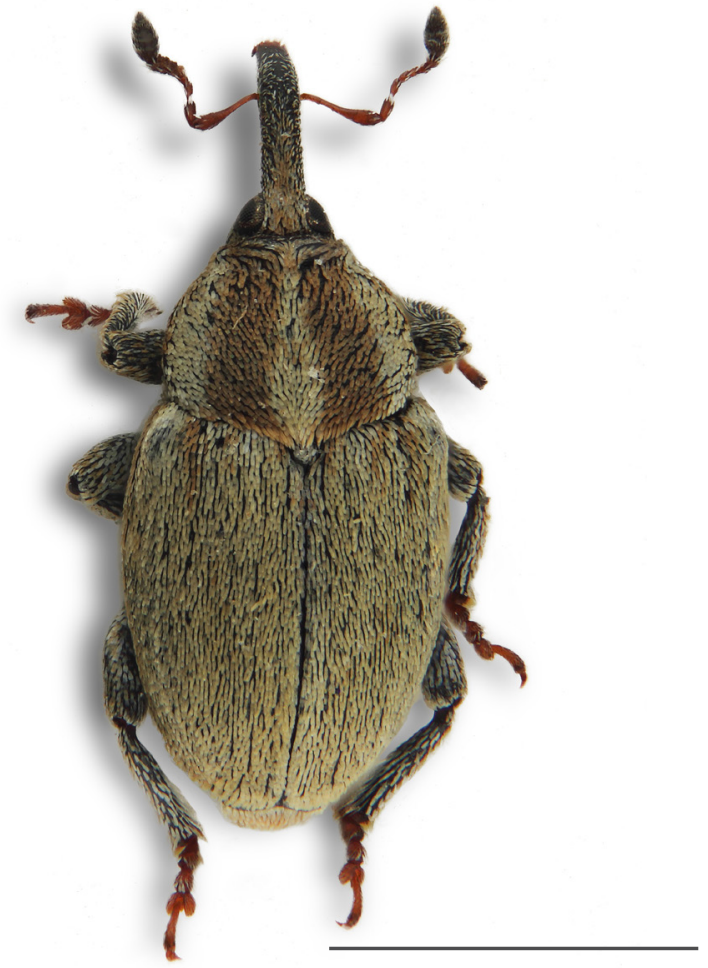

Fig. 16. Sibinia (Sibinia) femoralis Germar, 1824. Scale bar: $1 \mathrm{~mm}$. Photo Zoltán CsATA

(quarry), 7.VII.2014, leg. KI, OA, PA, SzL.

\section{Sibinia (Sibinia) phalerata (GYLlENHAL, 1835)}

Published data: Montandon 1887, 1908; FleCK 1905; NeGRU and RoşCa 1967; Raduta 2014

New data: Tulcea County: Greci, 22.VI.1963, leg. IS (Coll. Mus. G. ANTIPA) - Măcin (hillside, 200 m), 29.V.2005, leg.CsS;M-ţiiMăcin, ValeaFagilor (quarry), 30.V.2005, leg. CsS; Enisala (castle), 22.IX.2015, leg. KI, KJ, PA, 10.V.2017, leg. KI; Constanţa County: Cheia env., Cheia Nature Reserve, 19.IX.2010, leg. JK; $2 \mathrm{~km}$ from Sinoie to SE, 5.VI.2018, leg. FK, KI, KJ, PA; Sinoie, Grindul Lupilor, 5.VI.2018, leg. KI; Crucea, 9.VI.2018, leg. KI.

Sibinia (Sibinia) subelliptica (DESBROCHERS des LOGES, 1873)

New data: Constanţa County: Vadu, Grindul Chituc, 6.VI.2018, leg. FK, KI, KJ, PA.

\section{Sibinia (Sibinia) tibialis GYLLENHAL, 1835}

Published data: PerJu and TeOdor 1998

New data: Constanţa County: Agigea, 30.VII.1992, leg. AS; Rezervaţia Agigea, 18.VII.2003, leg. AP(Coll. Mus. G. ANTIPA) - Vadu, Grindul Chituc, 22.VI.2019, leg. KI, KJ, PA, TB.

Sibinia (Sibinia) unicolor FÅHRAEUS, 1843

Published data: TeOdor and Manole 1996, Raduta 2014

New data: Constanţa County: Sinoie, Grindul Lupilor, 27.VI.2017, leg. KI, KJ, PA, TB; Vadu, Grindul Chituc, 22.VI.2019, leg. KI, KJ, PA, TB; Sinoie, 
23.VI.2019, leg. KI.

*Sibinia (Sibinia) variata GYllenhal, 1835

New data: Constanţa County: Istria, Cetatea Histria, 14.IX.2016, leg. KI, KJ, PA, TB; $2 \mathrm{~km}$ from Sinoie to SE, 5.VI.2018, leg. FK, KI, KJ, PA.

\section{Sibinia (Sibinia) viscariae (LINNÉ, 1760)}

Published data: FLECK 1905

New data: Tulcea County: N from Nifon, 23.VI.2017, leg. KI, KJ, PA, TB; Jurilovca, 23.VI.2019, leg. KI, KJ, PA, TB; Constanţa County: Murfatlar, Fântânița, 22.V.2015. leg. PA, RI, SzK, SzL; Vadu, Grindul Chituc, 6.VI.2018, leg. KI.

\section{Sibinia (Sibinia) vittata GERMAR, 1823}

Published data: Jaquet 1899b, Fleck 1905, Negru and Roşca 1967, TEOdor and MANOLE 1996, RAduTA 2014

New data: Tulcea County: Slava Rusă, 11.V.2017, leg. FK, KI, KJ, PA; $1,5 \mathrm{~km}$ from Beidaud to SW, 25.VI.2017, leg. KI, KJ, PA, TB; Constanţa County: Agigea, 15.VI.1964, leg. XS; Agigea, 23.VI.1964, leg. MW (Coll. Mus. G. ANTIPA) - Murfatlar, Fântâniţa, 4-5.VII.2014, leg. KI, OA, PA, SzL, 7-8.VI.2018, leg. FK, KI, KJ, PA; Esechioi (forest), 7.VII.2014, leg. KI, OA, PA, SzL; Negureni, Pădurea Valea Cişmelelor, 23.V.2015, leg. PA, RI, SzK, SzL; Gura Dobrogei, 25-27.VI.2017, leg. KI, KJ, PA, TB, 7.VI.2018, leg. FK, KI, KJ, PA.

\section{Tychius (Tychius) astragali BECKER, 1862}

Published data: CALdara and Podlussány 2018

New data: Constanţa County: Gura Dobrogei, 28-29.

IV.2012, leg. KI.

Tychius (Tychius) aureolus KIESENWETTER, 1852 Published data: Montandon 1887, FleCK 1905 New data: Tulcea County: Codru Monastery, 30.IV3.V.2014, leg. KI, IZ, PA, SzL; Enisala, Cetatea Heraclea, 25.VI.2017, leg. KI, KJ, PA, TB; Enisala (castle), 20.VI.2019, leg. KI, KJ, PA, TB; Constanţa County: Murfatlar, Fântâniţa, 4-5.VII.2014, leg. KI, OA, PA, SzL; Esechioi (forest), 7.VII.2014, leg. KI, OA, PA, SzL; Oltina (village), 8.VII.2014, leg. KI, OA, PA, SzL.

\section{Tychius (Tychius) balcanicus CALDARA, 1990}

New data: Tulcea County: Codru Monastery, 2325.V.2013, leg. KI, PA, RI, SzL, 30.IV-3.V.2014, leg. KI, IZ, PA, SzL, 19.V.2015, leg. PA, RI, SzK, SzL, 10-11.V.2017, leg. FK, KI, KJ, PA, 4.VI.2018, leg. KI; Enisala, 24-25.V.2013, leg. KI, PA, RI, SzL, 29.IV3.V.2014, leg. KI, IZ, PA, SzL; Enisalai (castle), 21.V.2015, leg. PA, RI, SzK, SzL, 10.V.2017, leg. FK, KI, KJ, PA; M-ţii Măcin, Greci, 8.V.2017, leg. FK, KI, KJ, PA; Slava Rusă, 11.V.2017, leg. FK, KI, KJ, PA; S from Hamcearca, 24.VI.2017, leg. KI, KJ, PA, TB; Constanţa County: Balta Limanu, 5-6.VII.2014, leg. KI, OA, PA, SzL; Gura Dobrogei, 12.V.2017, leg. FK, KI, KJ, PA; Limanu, 12.V.2017, leg. FK, KI, KJ, PA; Albeşti, Pădurea Hagieni (forest), 12.V.2017, leg. FK, KI, KJ, PA.

\section{Tychius (Tychius) beckeri TOURNIER, 1874}

New data: Tulcea County: Codru Monastery, 2325.V.2013, leg. KI, PA, RI, SzL, 10-11.V.2017, leg. KI, 20.VI.2019, leg. KI, KJ, PA, TB; $2 \mathrm{~km}$ from Slava Rusă to NE, 3.VI.2018, leg. FK, KI, KJ, PA; Constanţa County: Esechioi (forest), 7.VII.2014, leg. KI, OA, PA, SzL.

Tychius (Tychius) breviusculus DESBROCHERS des LOGES, 1873

New data: Tulcea County: Codru Monastery, 2325.V.2013, leg. KI, PA, RI, SzL, 4.VI.2018, leg. FK, KI, KJ, PA; Enisala (castle), 20.VI.2019, leg. KI, KJ, PA, TB; Enisala (forest), 20.VI.2019, leg. KI, KJ, PA, TB; Jurilovca, 23.VI.2019, leg. KI, KJ, PA, TB; Constanţa County: Istria, Cetatea Histria, 21.V.2015, leg. PA, RI, SzK, SzL, 14.IX.2016, leg. KI, KJ, PA, TB, 26-28.VI.2017, leg. KI, KJ, PA, TB, 6.VI.2018, leg. FK, KI, KJ, PA; Vadu, Grindul Chituc, 22.VI.2019, leg. KI, KJ, PA, TB; Sinoie, Grindul Lupilor, 22.VI.2019, leg. KI, KJ, PA, TB; Sinoie, 21, 23.VI.2019, leg. KI, KJ, PA, TB.

\section{Tychius (Tychius) caldarai DIECKMANN, 1986}

New data: Constanţa County: Hârşova, leg. ALM (Coll. Mus. G. ANTIPA).

\section{Tychius (Tychius) cuprifer (PANZER, 1799)}

New data: Tulcea County: M-ţii Măcin, Valea Fagilor (quarry), 30.V.2005, leg. CsS; M-ţii Măcin, Luncaviţa, 30.V.2005, leg. CsS; M-ţii Măcin, Pricopan, 1.VI.2005, leg. CsS; Slava Rusă, 11.V.2017, leg. FK, KI, KJ, PA; S from Hamcearca, 24.VI.2017, leg. KI, KJ, PA, TB; $2,7 \mathrm{~km}$ from Horia to S, 28.VI.2017, leg. KI, KJ, PA, TB; M-ţii Măcin, 5 km S Greci, 8.V.2017, leg. KI; M-ţii Măcin, to the Peak Ţuţuiatu, 28.IV.2010, leg. TLA; Constanţa County: Esechioi (forest), 7.VII.2014, leg. KI, OA, PA, SzL.

\section{Tychius (Tychius) flavus BECKER, 1864}

Published data: IONEscu, Mitru and ŢurCa 1963, IONESCU, TOMOROGA and ȚURCA 1967

New data: Tulcea County: Beştepe, 13-14.VI.1993, leg. IM; Celic Dere, 20.V.1997, leg. CH (Coll. Mus. G. Antipa) - M-ţii Măcin, Valea Fagilor (quarry), 30.V.2005, leg. CsS; Codru Monastery, 23-25.V.2013, leg. KI, PA, RI, SzL, 20.VI.2019, leg. KI; Enisala, 24-25.V.2013, leg. KI, PA, RI, SzL; Constanţa County: Esechioi (forest), 7.VII.2014, leg. KI, OA, PA, SzL; $2 \mathrm{~km}$ from Sinoie to SE, 27.VI.2017, leg. KI; Istria, Cetatea Histria, 6.VI.2018, leg. FK, KI, KJ, PA; Murfatlar, Fântâniţa, 7-8.VI.2018, leg. FK, $\mathrm{KI}, \mathrm{KJ}, \mathrm{PA}$. 
Tychius (Tychius) grenieri C. N. F. BRISOUT de BARNEVILle, 1862

Published data: Montandon 1887, Fleck 1905

New data: Tulcea County: Codru Monastery, 30.IV3.V.2014, leg. KI, IZ, PA, SzL; Enisala (castle), 21.V.2015, leg. PA, RI, SzK, SzL, 10.V.2017, leg. FK, KI, KJ, PA; Enisala (wooded pasture), 4.VI.2018, leg. FK, KI, KJ, PA; Constanţa County: Murfatlar, Fântâniţa, 4-5.VII.2014, leg. KI，OA，PA，SzL; Palazu Mic, 22.V.2015, leg. PA, RI, SzK, SzL.

\section{Tychius (Tychius) junceus (REICH, 1797)}

Published data: Montandon 1887, Fleck 1905, Negru and Roşca 1967, Raduta 2014

New data: Tulcea County: Frecăţei, Celic Dere Monastery (hillside) 31.V.2005, leg. CsS; Babadag (forest), 23-25.V.2013, leg. KI, PA, RI, SzL; Codru Monastery, 23-25.V.2013, leg. KI, PA, RI, SzL, 10 11.V.2017, leg. FK, KI, KJ, PA, 20.VI.2019, leg. KI, KJ, PA, TB; Smârdan, Braţul Măcin, 22.VI.2017, leg. KI, KJ, PA, TB, 18.VI.2019, leg. KI, KJ, PA, TB; Enisala, Cetatea Heraclea, 25.VI.2017, leg. KI, KJ, PA, TB; 2 km from Slava Rusă to NE, 3.VI.2018, leg. FK, KI, KJ, PA; Turcoaia, $6 \mathrm{~km}$ from Cerna to NW, 19.VI.2019, leg. KI, KJ, PA, TB; Enisala (castle), 20.VI.2019, leg. KI, KJ, PA, TB; Enisala (forest), 20.VI.2019, leg. KI, KJ, PA, TB; Constanţa County: Murfatlar, Fântâniţa, 4-5.VII.2014, leg. KI, OA, PA, SzL; Esechioi (forest), 7.VII.2014, leg. KI, OA, PA, SzL; Istria, Cetatea Histria, 26-28.VI.2017, leg. KI, KJ, PA, TB; Vadu, Grindul Chituc, 6.VI.2018, leg. KI, 22.VI.2019, leg. KI, KJ, PA, TB; Sinoie, Grindul Lupilor, 22.VI.2019, leg. KI, KJ, PA, TB.

\section{Tychius (Tychius) kulzeri PENECKe, 1934}

New data: Tulcea County: Codru Monastery, 2325.V.2013, leg. KI, PA, RI, SzL; Smârdan, Braţul Măcin, 2.VI.2018, leg. FK, KI, KJ PA; Enisala (castle), 20.VI.2019, leg. KI, KJ, PA, TB; Constanţa County: Istria, Cetatea Histria, 28.VI.2017, leg. KI, KJ, PA, TB; Murfatlar, Fântâniţa, 7-8.VI.2018, leg. FK, KI, KJ, PA; Sinoie, 21, 23.VI.2019, leg. KI.

\section{Tychius (Tychius) longulus DESBROCHERS des} Loges, 1873

Published data: Caldara and Podlussány 2018

New data: Constanţa County: Sinoie, Grindul Lupilor, 27.VI.2017, leg. KI, KJ, PA, TB, 5.VI.2018, leg. FK, KI, KJ, PA, 22.VI.2019, leg. KI, KJ, PA, TB; Istria, Cetatea Histria, 6.VI.2018, leg. KI; Vadu, Grindul Chituc, 6.VI.2018, leg. KI.

Tychius (Tychius) medicaginis C. N. F. BRISOUT de BARNEVILLE, 1863

Published data: Montandon 1887, FleCK 1906

New data: Tulcea County: Celic Dere, 20.V.1997, leg. CH (Coll. Mus. G. ANTIPA) - M-ţii Măcin, Valea Fagilor (quarry), 30.V.2005, leg. CsS; Babadag (forest), 2.VI.2005, leg. CsS, 23-25.V.2013, leg. KI,
PA, RI, SzL; Codru Monastery, 23-25.V.2013, leg. KI, PA, RI, SzL, 19.V.2015, leg. PA, RI, SzK, SzL, 10-11.V.2017, leg. FK, KI, KJ, PA, 24-25.VI.2017, leg. KI, KJ, PA, TB, 4.VI.2018, leg. KI, 20.VI.2019, leg. KI, KJ, PA, TB; Slava Rusă, 24-25.V.2013, leg. KI, PA, RI, SzL, 1-3.V.2014, leg. KI, IZ, PA, SzL, 11.V.2017, leg. FK, KI, KJ, PA; Turcoaia, 6 $\mathrm{km}$ from Cerna to NW, 24.VI.2017, leg. KI, KJ, PA, TB, 3.VI.2018, leg. FK, KI, KJ, PA, 19.VI.2019, leg. KI, KJ, PA, TB; S from Hamcearca, 24.VI.2017, leg. KI, KJ, PA, TB; $1,5 \mathrm{~km}$ from Beidaud to SW, 25.VI.2017, leg. KI, KJ, PA, TB; Enisala, Cetatea Heraclea, 25.VI.2017, leg. KI, KJ, PA, TB; 2 km from Slava Rusă to NE, 3.VI.2018, leg. FK, KI, KJ, PA; Enisala (wooded pasture), 4.VI.2018, leg. FK, KI, KJ, PA, 20.VI.2019, leg. KI, KJ, PA, TB; Enisala (castle), 20.VI.2019, leg. KI, KJ, PA, TB; Constanţa County: Murfatlar, Fântâniţa, 4-5.VII.2014, leg. KI, OA, PA, SzL, 22.V.2015, leg. PA, RI, SzK, SzL, 7-8.VI.2018, leg. FK, KI, KJ, PA; Esechioi (forest), 7.VII.2014, leg. KI, OA, PA, SzL; Oltina (village), 8.VII.2014, leg. KI, OA, PA, SzL; Sinoie, Grindul Lupilor, 5.VI.2018, leg. FK, KI, KJ. PA.

\section{Tychius (Tychius) meliloti STEPHENS, 1831}

Published data: Montandon 1887, Fleck 1905

New data: Tulcea County: Măcin (hillside, 200 m), 29.V.2005, leg. CsS; M-ţii Măcin, Valea Fagilor (quarry), 30.V.2005, leg. CsS; Smârdan, Braţul Măcin, 7.V.2017, leg. FK, KI, KJ, PA; Codru Monastery, 1011.V.2017, leg. FK, KI, KJ, PA, 4.VI.2018, leg. KI, 20.VI.2019, leg. KI, KJ, PA, TB; Nifon, pârâul Pârlita valley, 23.VI.2017, leg. KI, KJ, PA, TB; S from Hamcearca, 24.VI.2017, leg. KI, KJ, PA, TB; Enisala (castle), 10.V.2017, leg. FK, KI, KJ, PA, 20.VI.2019, leg. KI, KJ, PA, TB; 2 km from Slava Rusă to NE, 3.VI.2018, leg. FK, KI, KJ, PA; Enisala (wooded pasture), 4.VI.2018, leg. FK, KI, KJ, PA, 20.VI.2019, leg. KI, KJ, PA, TB; Jurilovca, 23.VI.2019, leg. KI; Constanţa County: Tuzla, 1.VIII.1992, leg. AP (Coll. Mus. G. AnTIPA) - Esechioi (forest), 7.VII.2014, leg. KI, OA, PA, SzL; Băneasa, Canaraua Fetii (quarry), 7.VII.2014, leg. KI, OA, PA, SzL; Palazu Mic, 22.V.2015, leg. PA, RI, SzK, SzL; Neptun-Olimp, Pădurea Comorova, 17.IX.2016, leg. FK, KI, KJ, PA; Gura Dobrogei, 25-27.VI.2017, leg. KI, KJ, PA, TB; Istria, Cetatea Histria, 26-28.VI.2017, leg. KI, KJ, PA, TB, 21.VI.2019, leg. KI, KJ, PA, TB; Sinoie, Grindul Lupilor, 27.VI.2017, leg. KI, KJ, PA, TB, 5.VI.2018, leg. FK, KI, KJ, PA, 22.VI.2019, leg. KI, KJ, PA, TB; $2 \mathrm{~km}$ from Sinoie to SE, 27.VI.2017, leg. KI, KJ, PA, TB; Crucea, 9.VI.2018, leg. FK, KI, KJ, PA; Vadu, Grindul Chituc, 22.VI.2019, leg. KI, KJ, PA, TB.

*Tychius (Tychius) ochraceus TOURNIER, 1873 (fig. 17) New data: Tulcea County: Smârdan, Braţul Măcin, 22.VI.2017, leg. KI, 2.VI.2018, leg. FK, KI, KJ, PA; Constanţa County: Esechioi (forest), 7.VII.2014, leg. 
KI, OA, PA, SzL; $2 \mathrm{~km}$ from Sinoie to SE, 27.VI.2017, leg. KI, KJ, PA, TB, 5.VI.2018, leg. FK, KI, KJ, PA; Sinoie, Grindul Lupilor, 27.VI.2017, leg. KI, PA, TB.

Tychius (Tychius) picirostris (FABRICIUS, 1787)

Published data: Teodor and Manole 1996, Raduta 2014

New data: Tulcea County: M-ţii Măcin,Valea Fagilor (dried lake bed), 30.V.2005, leg. CsS; M-ţii Măcin,Valea Fagilor (quarry), 30.V.2005, leg. CsS; Frecăţei, Celic Dere Monastery (hillside), 31.V.2005, leg. CsS; M-ţii Măcin, 5 km S Greci, 8.V.2017, leg. FK, KI, KJ, PA, 18-19.VI.2019, leg. KI, KJ, PA, TB; Jurilovca, 23.VI.2019, leg. KI, KJ, PA, TB; Constanţa County: Gura Dobrogei, 30.IV-2.V.2014, leg. KI, IZ, PA, SzL; Esechioi (forest), 7.VII.2014, leg. KI, OA, PA, SzL; Palazu Mic, 22.V.2015, leg. PA, RI, SzK, SzL; Murfatlar, Fântâniţa, 22.V.2015, leg. PA, RI, SzK, SzL.

\section{Tychius (Tychius) polylineatus (GERMAR, 1823)}

New data: Tulcea County: S of Horia, 6.V.2009, leg. JK; M-ţii Măcin, Hamcearca, Culmea Cardonului, 19.V.2017, leg. CS, TLA.

Tychius (Tychius) pumilus C. N. F. BRISOUT de BARNEVILLE, 1863

New data: Constanţa County: Esechioi (forest), 7.VII.2014, leg. KI, OA, PA, SzL.

Tychius (Tychius) quinquepunctatus (LINNÉ, 1758) Published data: MONTANDON 1887, JAQUET 1899b, Fleck 1905, Negru and RoşCa 1967, Raduta 2014 New data: Tulcea County: Babadag, Visterna, 1516.V.1963, leg. IC; Celic Dere, 20.V.1997, leg. CH, 24.V.1997, leg. CP, 27.V.2005, leg. RS (Coll. Mus. G. ANTIPA) - Slava Rusă, 9.V.2017, leg. FK, KI, KJ, PA, 11.V.2017, leg. FK, KI, KJ, PA; Slava Rusă, Uspenia Monastery, 11.V.2017, leg. FK, KI, KJ, PA; Nifon, pârâul Pârlita valley, 23.VI.2017, leg. KI, KJ, PA, TB; S from Hamcearca, 24.VI.2017, leg. KI; 2 km from Slava Rusă to NE, 3.VI.2018, leg. FK, KI, KJ, PA; Horea, 3.VI.2018, leg. KI; Constanţa County: Băneasa, Canaraua Fetii, 20.VII.1958, leg. IS; Agigea, 20.VI.1960, leg. MW; Băneasa, 30.V.1965, leg. MW; Negureni, 25.VI.1995, leg. CP (Coll. Mus. G. ANTIPA) - Esechioi (forest), 7.VII.2014, leg. KI, OA, PA, SzL; Oltina (village), 8.VII.2014, leg. KI; Cochirleni, 8.VII.2014, leg. KI; Murfatlar, Fântâniţa, 22.V.2015, leg. PA, RI, SzK, SzL; Băneasa, Canaraua Fetii, 23.V.2015 leg. PA, RI, SzK, SzL; Limanu, 12.V.2017, leg. FK, KI, KJ, PA.

Tychius (Tychius) rufipennis C. N. F. BRISOUT de BARNEVILLE, 1863

New data: Tulcea County: 1,5 km from Beidaud to SW, 25.VI.2017, leg. KI, KJ, PA, TB.

Tychius (Tychius) russicus DesBrochers des Loges,
1908

New data: Tulcea County: Enisala, 29.IV-3.V.2014, leg. KI, IZ, PA, SzL; Enisala (castle), 10.V.2017, leg. FK, KI, KJ, PA; Constanţa County: $3 \mathrm{~km} \mathrm{~W}$ of Crucea, Allah Bair (Nature Reserve), 1.V.2009, leg. JK; Gura Dobrogei, 30.IV-2.V.2014, leg. KI, IZ, PA, SzL, 12.V.2017, leg. FK, KI, KJ, PA.

\section{Tychius (Tychius) schneideri (HERBST, 1795)}

New data: Tulcea County: Babadag (forest), 2005. VI.2, leg. CsS.

\section{Tychius (Tychius) sharpi TOURNIER, 1874}

New data: Tulcea County: Smârdan, Braţul Măcin, 18.VI.2019, leg. KI.

\section{Tychius (Tychius) squamulatus GYLLENHAL, 1835}

Published data: MONTANDON 1887; JAQUET 1899b, 1900a; Fleck 1905; Negru and RoşCa 1967; Raduta 2014

New data: Tulcea County: $2,7 \mathrm{~km}$ from Horia to $\mathrm{S}$, 28.VI.2017, leg. KI, KJ, PA, TB; Codru Monastery, 23-25.V.2013, leg. KI, PA, RI, SzL, 10-11.V.2017, leg. FK, KI, KJ, PA; Constanţa County: $2 \mathrm{~km}$ from Sinoie to SE, 27.VI.2017, leg. KI, KJ, PA, TB; Istria, Cetatea Histria, 26.VI.2017, leg. KI, KJ, PA, TB, 6.VI.2018, leg. KI; Sinoie, Grindul Lupilor, 22.VI.2019, leg. KI, KJ, PA, TB.

Tychius (Tychius) stephensi SCHÖNHERR, 1835 Published data: Montandon 1887, FLECK 1905 New data: Constanţa County: Agigea, 9.VIII.1962, leg. ID (Coll. Mus. G. ANTIPA).

\section{*Tychius (Tychius) subsulcatus TOURNIER, 1874 (fig. 18)}

New data: Tulcea County: Babadag (forest), 2325.V.2013 leg. KI, PA, RI, SzL; 2 km from Slava Rusă to NE, 3.VI.2018, leg. FK, KI, KJ, PA; Codru Monastery, 4.VI.2018, leg. FK, KI, KJ, PA.

\section{Tychius (Tychius) tibialis BOHEMAN, 1843}

New data: Tulcea County: Celic Dere, 20. 05. 1997, leg. CH (Coll. Mus. G. AnTIPA) - Frecăţei, Celic Dere Monastery (hillside), 31.V.2005, leg. CsS; Stejaru env., 5.V.2009, leg. JK; M-ţii Măcin, 5 km S Greci, 8.V.2017, leg. KI; Măcin, Rezervaţia de la Greci, 23.IV.2009, leg. TLA; M-ţii Măcin, to the Peak Ţuţuiatu, 28.IV.2010, leg. TLA; Slava Rusă, 1-3.V.2014, leg. KI, IZ, PA, SzL; Codru Monastery, 10-11.V.2017, leg. FK, KI, KJ, PA; Enisala, Cetatea Heraclea, 25.VI.2017, leg. KI, KJ, PA, TB; S from Hamcearca, 24.VI.2017, leg. KI, KJ, PA, TB; 2,5 km from Stejaru to NW, 25.VI.2017, leg. KI, KJ, PA, TB; M-ţii Măcin, Greci, 18-19.VI.2019, leg. KI, KJ, PA, TB; Constanţa County: Gura Dobrogei, 26.V.2013, leg. KI, PA, RI, SzL; Esechioi (forest), 7.VII.2014, leg. KI, OA, PA, SzL; Hagieni, Pădurea Hagieni (forest), 17.IX.2016, leg. FK, KI, KJ, PA. 


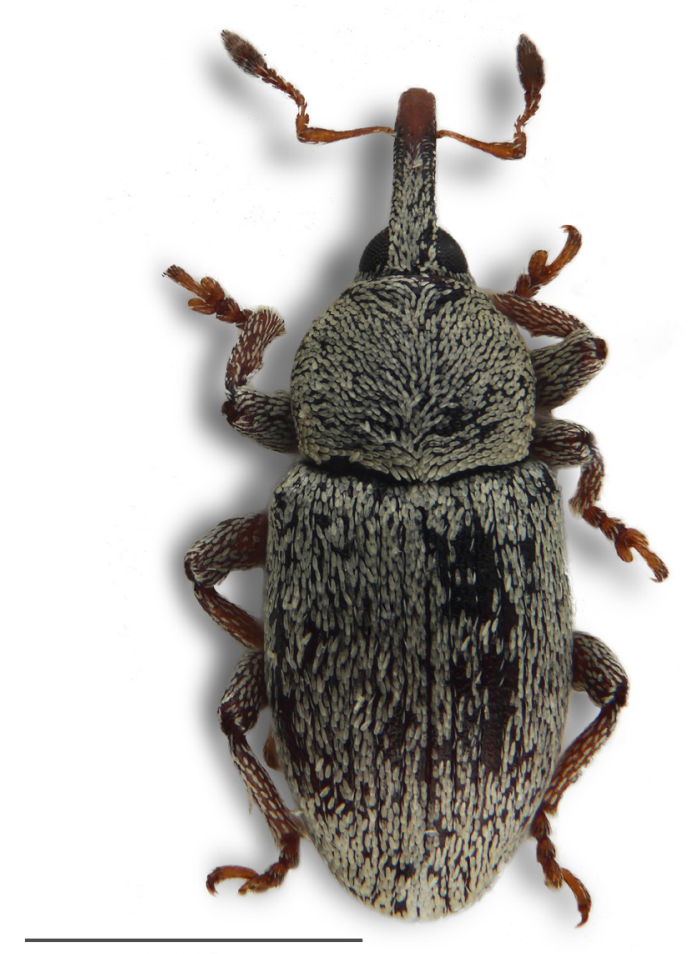

Fig. 17. Tychius (Tychius) ochraceus TourNIER, 1873. Scale bar: $1 \mathrm{~mm}$. Photo Zoltán CsaTA

Tychius (Tychius) trivialis BOHEMAN, 1843

New data: Tulcea County: Slava Rusă, 24-25.V.2013, leg. KI, PA, RI, SzL, 1-3.V.2014, leg. KI, IZ, PA, SzL, 9.V.2017, leg. FK, KI, KJ, PA; 2,5 km from Stejaru to NW, 25.VI.2017, leg. KI, KJ, PA, TB.

CYCLOMINAE SCHÖNHERR, 1826

Hipporhinini LACORDAIRE, 1863

Gronops (Asperogronops) sulcatus BOHEMAN, 1842 Published data: Procheş 1999

New data: Constanţa County: Vadu, Grindul Chituc, 22.VI.2019, leg. PA.

Entomoderus (Byrsopidius) deformis (REICHE, 1858) Published data: JaQuet 1900b, FleCK 1905, Osella and Magnano 1984, Procheş 1999

Entomoderus (Byrsopidius) geticus (OSELLA, 1973) Published data: Osella, 1973

Entomoderus (Byrsopidius) phrygius (K. DANIEL \& J. DANIEL, 1903)

Published data: Montandon 1906, 1908; Negru and Roşca 1967; RADUTA 2014

New data: Tulcea County: „Babadag, Dobrutscha, Breit”; „Dobrudja, Măcin”, leg. ALM; „Dobrudja, Măcin-Iglitea”, leg. ALM (Coll. Hung. Nat. Hist. Mus.).

RHYNCHOPHORINAE SCHÖNHERR, 1833

Dryophthorini SCHÖNHERR, 1825

Dryophthorus corticalis (PAYKULL, 1792)

New data: Tulcea County: Caraorman, 16.VI.1969,

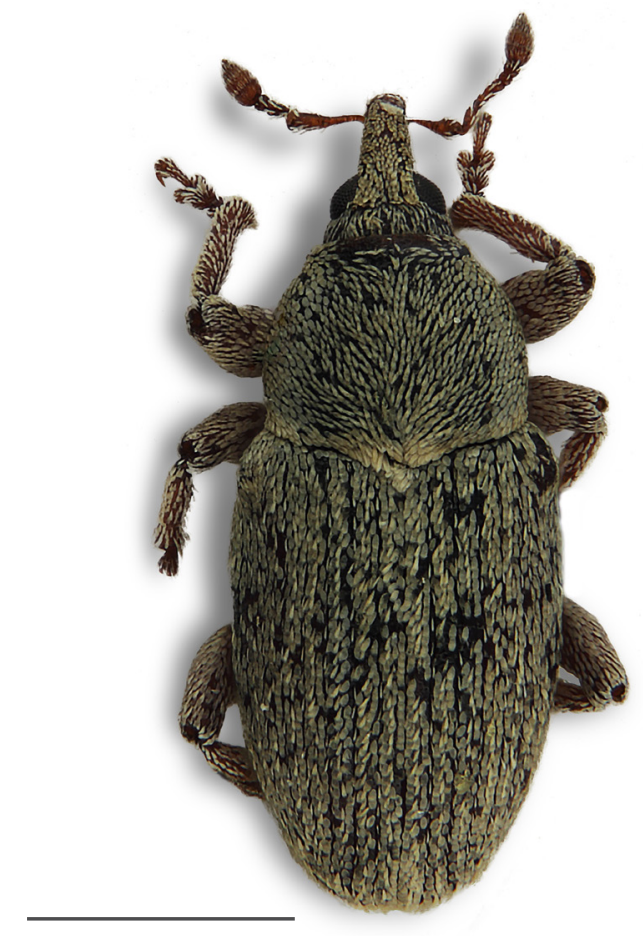

Fig. 18. Tychius (Tychius) subsulcatus TOURNIER, 1874. Scale bar: $1 \mathrm{~mm}$. Photo Zoltán CSATA

leg. ŞN, (Coll. Mus. G. ANTIPA).

Rhynchophorini SCHÖNHERR, 1833

Litosomina LACORDAIRE, 1865

Sitophilus granarius (LINNÉ, 1758)

Published data: Jaquet 1898c; Fleck 1905; Negru 1957, 1968; Negru and RoşCa 1967; Raduta 2014

Sitophilus oryzae (LinNÉ, 1763)

New data: Tulcea County: Măcin, leg. ALM (Coll. Mus. G. Antipa) - Măcin, 7.V.2017, leg. KI; Constanţa County: Valu lui Traian, 16.VII.1955, leg. XS (Coll. Mus. G. ANTIPA).

Sitophilus zeamais MotsCHULSKY, 1855

Published data: PANIN 1951

New data: Tulcea County: 2,5 km from Stejaru to NW, 25.VI.2017, leg. KI, KJ, PA, TB.

Sphenophorina Lacordaire, 1865

Sphaenophorus abbreviatus (FABRICIUS, 1787)

Published data: FLECK 1905; MONTANDON 1908; Negru 1957, 1968; Negru and RoşCa 1967; TeOdor and Manole 1996; Raduta 2014

New data: Tulcea County: Periprava, 7.X.1963, leg. XS (Coll. Mus. G. ANTIPA); Constanţa County: Băneasa, Canaraua Fetii, 19.V.1994, leg. CP (Coll. Mus. G. ANTIPA) - Istria, Cetatea Histria, 26.VI.2017, leg. TB, 21.VI.2019, leg. TB.

Sphaenophorus piceus (PALLAS, 1771)

Published data: Montandon 1887, JAQUet 1899b, Fleck 1905, Negru and RoşCa 1967, Negru 1968, 
Teodor 1993, Teodor and Manole 1996, Raduta 2014

New data: Tulcea County: C. A. Rosetii, VII.1950, leg. IC; Babadag, 29.VI.1956, leg. XS; Periprava, 23.VI.1962, leg. NS, 18.VII.1964, leg. XS, 2.VII.1965, leg. ŞN; Sarinasuf, 24.VI.1962, leg. XS; Băneasa, Canaraua Fetii, 11.VI.1964, leg. NS; C. A. Rosetti, Delta Dunării, 3.VI.1981, leg. XS; Letea, 14.VII.1981, leg. AB; Caraorman, 26.VI.1991, leg. IP, GA; Enisala, 28.VI.1991, leg. GA (Coll. Mus. G. Antipa); Constanţa County: Mangalia, 13.VI.1948, leg. XS; Hagieni, 29.V.1963. leg. NS (Coll. Mus. G. ANTIPA) - Sinoie, 23.VI.2019, leg. KI.

Sphaenophorus striatopunctatus (GoEZE, 1777)

Published data: TeOdor 1993, RAduta 2014

New data: Tulcea County: Luncaviţa, Parcul Naţional M-ţii Măcin, 24.V.2005, leg. MS (Coll. Mus. G. ANTIPA).

ENTIMINAE SCHÖNHERR, 1823

Brachyderini SCHÖNHERR, 1826

Strophomorphus porcellus (SCHÖNHERR, 1832)

Published data: Montandon 1908, Negru and RoşCa 1967, RADUTA 2014

New data: Constanţa County: Agigea, 27.V.1963, leg. XS (Coll. Mus. G. ANTIPA) - Balta Limanu, 5-6. VII.2014, leg. KI, OA, PA, SzL; Limanu, 12.V.2017, leg. FK, KI, KJ, PA; Murfatlar, Fântâniţa, 7-8. VI.2018, leg. FK, KI, KJ, PA; Istria, Cetatea Histria, 21.VI.2019, leg. KI.

Byrsopagini LACORDAIRE, 1863

\section{Graptus kaufmanni STIERLIN, 1884}

New data: Constanţa County: Murfatlar, X.1951, leg. NS; Agigea, 29.V.1963, leg. XS (Coll. Mus. G. ANTIPA) - Murfatlar, Fântâniţa, 7-8.VI.2018, leg. FK, KI, KJ, PA.

\section{Graptus triguttatus (FABRICIUS, 1775)}

Published data: Montandon 1887, Fleck 1905

New data: Tulcea County: Revărsarea, 23.V.1997, leg. RS (Coll. Mus. G. ANTIPA) - Nifon, pârâul Pârlita valley, 23.VI.2017, leg. KI, KJ, PA, TB; Constanţa County: Hagieni, 29.V.1963, leg. NS; Furnica, Rezervaţia Dumbrăveni, 13-14.V.1995, leg. CP (Coll. Mus. G. ANTIPA).

No data in Alonso-Zarazaga et al. 2017 for Romania, although this species has many reports from various regions of Romania.

Tropiphorus elevatus (HERBST, 1795)

New data: Tulcea County: M-ţii Măcin, Nifon (forest), 23.VI.2017, leg. KI.

\section{Tropiphorus micans BOHEMAN, 1842}

New data: Tulcea County: NE of Topolog, 4.V.2009, leg. JK.
Cyphicerini LaCORDAIRE, 1863

Mylacorrhinina ReITTER, 1913

*Ptochus (Ptochus) porcellus Boheman, 1834 (fig. 19) New data: Constanţa County: - Hârşova, leg. ALM (Coll. Mus. G. ANTIPA) - Istria, Cetatea Histria, 14.IX.2016, leg. TB, 26-28.VI.2017, leg. KI, 21.VI.2019, leg. KI; Sinoie, Grindul Lupilor, 5.VI.2018, leg. FK, KI, KJ, PA.

\section{Geonemini Gistel, 1848}

Barynotus montandoni PIC, 1899

Published data: MonTANDON 1908

Myorhinini Marseul, 1863

Apsis albolineata (FABRICIUS, 1792)

Published data: JAQUET 1899a, 1899c, 1902b; FleCK

1905; Montandon 1908; IEniştea 1968; TeOdor 1993; TeOdor and Manole 1996; Raduta 2014 New data: Tulcea County: Greci, 22.VI.1963, leg. IS; C. A. Rosetti, Deltă, 10.V.1964, leg. MW, 10.V.1964, leg. XS, 11.V.1964, leg. MW; Rosetti, Pădurea Letea, 12.V.1964, leg. XS; Periprava, Letea, 13.V.1964, leg. XS; Caraorman, 31.V.1968, leg. MW, 03.VI.1968, leg. ID; Măcin, Valea Suluc, 27.V.2005, leg. CB, 27.V.2005, leg. IM (Coll. Mus. G. ANTIPA) - Dealul Taşburun, 2.V.1993, leg. TLA; Iancina, 11.VI.1993, leg. TLA; Beştepe, 14.VI.1993, leg. TLA; Enisala, 24-25.V.2013, leg. KI, PA, RI, SzL, 29.IV-3.V.2014, leg. KI, IZ, PA, SzL; Enisala (castle), 21.V.2015, leg. PA, RI, SzK, SzL, 10.V.2017, leg. FK, KI, KJ, PA; Enisala (wooded pasture), 4.VI.2018, leg. FK, KI, KJ, PA; Constanţa County: Hârşova, leg. ALM; Agigea, 22.VI.1964, leg. MW (Coll. Mus. G. ANTIPA) - Istria, Cetatea Histria, 2-4.V.2009, leg. JK, 21.V.2015, leg. PA, RI, SzK, SzL; Sinoie, Grindul Lupilor, 5.VI.2018, leg. FK, KI, KJ, PA.

Omiini Shuckard, 1840

Amicromias sp. nov. (det. YUNAKOv), the description of this species is in progress.

New data: Constanţa County: Murfatlar, Fântâniţa, 4-5.VII.2014, leg. KI, OA, PA, SzL, 7-8.VI.2018, leg. FK, KI, KJ, PA.

Eurosphalmus sp. nov., the description of this species is in progress.

(assimilated in/with? Brachysomus polonicus and $B$. podolicus sp.?) (det. Yunakov)

New data: Constanţa County: Gura Dobrogei, 30.IV2.V.2014, leg. KI, IZ, PA, SzL, 7.VI.2018, leg. FK, KI, KJ, PA.

\section{Eurosphalmus (Eurosphalmus) breiti (FORMÁNEK,} 1909)

Published data: YunAKov and NADEIN 2006

New data: Tulcea County: „Klst. Kokos Dobrutscha, Breit" (Coll. Hung. Nat. Hist. Mus.) M-ţii Măcin, Nifon (forest), 23.VI.2017, leg. KI. 


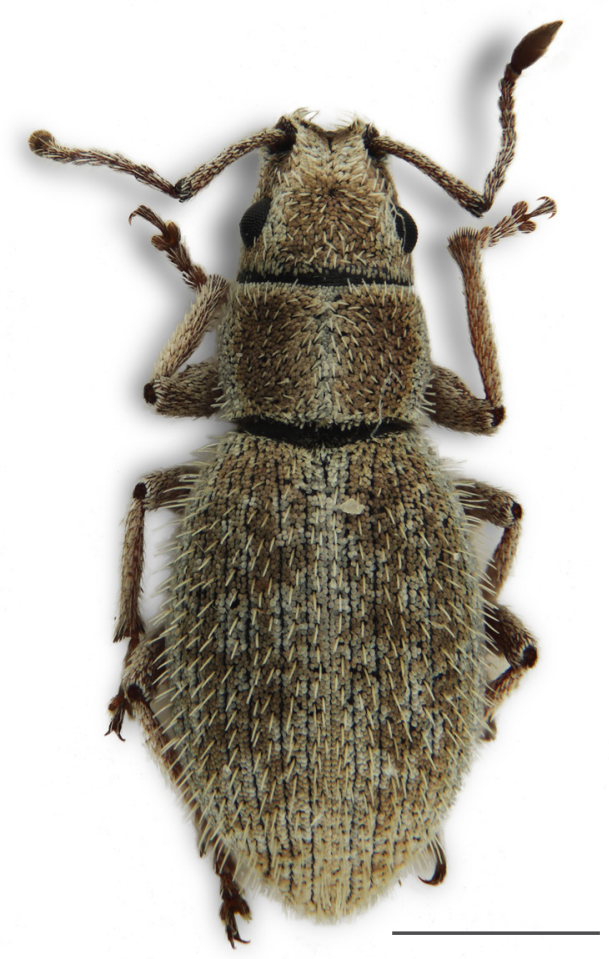

Fig. 19. Ptochus (Ptochus) porcellus Boheman, 1834. Scale bar: $1 \mathrm{~mm}$. Photo Zoltán CsATA

Omias globulus (BOHEMAN, 1842)

New data: Tulcea County: Periprava, 8.V.1964, leg. XS, 8.V.1964, leg. MW, 9.V.1964, leg. XS, 13.V.1964, leg. XS; Pădurea Letea, Deltă, 9.V.1964, leg. IS; C. A. Rosetti, Pădurea Letea, 11.V.1964, leg. XS (Coll. Mus. G. ANTIPA) - M-ţii Măcin, to the Peak Ţuţuiatu, 28.IV.2010, leg. TLA; Constanţa County: Istria, Cetatea Histria, 21.V.2015, leg. PA, RI, SzK, SzL.

*Omias murinus (BoHeMAN, 1842)

New data: Constanţa County: Istria env., Cetatea Histria, 2-4.V.2009, leg. JK.

Omias puberulus BOHEMAN, 1834 (= Mylacus rotundatus FABRICIUS 1792)

Published data: JAQUET 1902b, FleCK 1905, MONTANDON 1908

New data: Tulcea County: Enisala, 24-25.V.2013, leg. KI, PA, RI, SzL, 29.IV-3.V.2014, leg. KI, IZ, PA, SzL; Babadag (forest), 2005.VI.2, leg. CsS, 30.IV.2014, leg. KI, IZ, PA, SzL; Slava Rusă, 1-3.V.2014, leg. KI, 31.III.2016, leg. FK, KI, PA, SzL; Codru Monastery, 30-31.III.2016, leg. FK, KI, PA, SzL, 10-11.V.2017, leg. FK, KI, KJ, PA; Enisala (castle), 10.V.2017, leg. FK, KI, KJ, PA; Constanţa County: Furnica, Rezervaţia Dumbrăveni, 13-14. 05. 1995, leg. CP (Coll. Mus. G. Antipa) - Cetatea Histria, 2-4.V.2009, leg. JK; Gura Dobrogei, 30.IV-2.V.2014, leg. KI, IZ, PA, SzL, 12.V.2017, leg. FK, KI, KJ, PA; Cochirleni, 3.IV.2016, leg. FK, KI, PA, SzL; Albeşti, Pădurea Hagieni (forest), 12.V.2017, leg. FK, KI, KJ, PA; 23 August, 13.V.2017, leg. FK, KI, KJ, PA; Costineşti (coast), 13.V.2017, leg. KI.
Omias seminulum (FABRICIUS, 1792)

Published data: TeOdor 1993

New data: Tulcea County: Beștepe, 30.IV.1993, leg. TLA; Pădurea Letea (forest), 17.06.1993, leg. TLA; Dealul Taşburun, 2.V.1993, leg. TLA.

Otiorhynchini Schönherr, 1826

Dodecastichus contractus (STIERLIN, 1861)

New data: Constanţa County: Pădurea Hagieni, 10.VI.1975, leg. RS (Coll. Mus. G. ANTIPA).

Otiorhynchus (Arammichnus) hystrix GYLLENHAL, 1834 (= mandibularis W. REDTENBACHER, 1842) Published data: Fleck 1905, Montandon 1908, Negru and Roşca 1967, Raduta 2014

New data: Tulcea County: M-ţii Măcin, Hamcearca, 6.V.2009, leg. JK; Codru env., 23.IX.2010, leg. JK; Enisala, 24-25.V.2013, leg. KI, PA, RI, SzL; Codru Monastery, 30.IV-3.V.2014, leg. KI, IZ, PA, SzL, 19.V.2015, leg. PA, RI, SzK, SzL, 30-31.III.2016, leg. FK, KI, PA, SzL, 13.IX.2016, leg. KI, KJ, PA, TB; Enisala,22.IX.2015, leg. KI, KJ, PA, 13.IX.2016, leg. KI, KJ, PA, TB; M-ţii Măcin, 5 km S Greci, 8.V.2017, leg. FK, KI, KJ, PA; Slava Rusă, 11.V.2017, leg. KI; Constanţa County: Agigea, 20.IX.1964, leg. ŞN (Coll. Mus. G. ANTIPA) - Corbu env., 20.IX.2010, leg. JK; Istria env., Cetatea Histria, 22.IX.2010, leg. JK; Murfatlar, Fântâniţa, 14.IX.2016, leg. KI, KJ, PA, $\mathrm{TB}$

Otiorhynchus (Aranihus) ligneus ligneus (OLIVIER, 1807)

Published data: MonTANDON 1887

Otiorhynchus (Choilisanus) balcanicus STIERLIN, 1861

Published data: Montandon 1908

New data: Tulcea County: Codru Monastery, 2325.V.2013, leg. KI, PA, RI, SzL, 30-31.III.2016, leg. FK, KI, PA, SzL, 13.IX.2016, leg. KI, KJ, PA, TB; Babadag (forest), 30.IV.2014, leg. KI, IZ, PA, SzL; Enisala (castle), 29.IV-3.V.2014, leg. KI, IZ, PA, SzL.

\section{Otiorhynchus (Choilisanus) brunneus GYLLENHAL,} 1834

Published data: Montandon 1908, Negru 1957, Negru and Roşca 1967, Raduta 2014

New data: Tulcea County: Babadag (forest), 10.V.1992, leg. TLA, 13.IX.2016, leg. KI, KJ, PA, TB; Beştepe, 30.IV.1993, leg. TLA; Codru env., 23.IX.2010, leg. JK; Codru Monastery, 30.IV3.V.2014, leg. KI, IZ, PA, SzL, 19.V.2015, leg. PA, RI, SzK, SzL, 13.IX.2016, leg. KI, KJ, PA, TB; Slava Rusă, 1-3.V.2014, leg. KI, IZ, PA, SzL; Enisala (castle), 13.IX.2016, leg. KI, KJ, PA, TB, 20.VI.2019, leg. KI, KJ, PA, TB; Enisala (wooded pasture), 4.VI.2018, leg. KI; Constanţa County: Agigea, 7-10. VIII.1962, leg. ID, 26.VII.1963, leg. ŞN, 30.VIII.1963, leg. AtR, 3.IX.1963, leg. ŞN, 20.IX.1964, leg. ŞN, 
27.VII.1965, leg. ŞN; Hagieni, 28.VIII.1964, leg. NS (Coll. Mus. G. ANTIPA); Mangalia, leg. ALM (Coll. Hung. Nat. Hist. Mus.) $-3 \mathrm{~km}$ W of Crucea, Allah Bair (Nature Reserve), 1.V.2009, leg. JK, 18.IX.2010, leg. JK; Murfatlar, Fântâniţa, 24-25.IX.2015, leg. KI, KJ, PA, 14.IX.2016, leg. KI, KJ, PA, TB; Bugeac, 16.IX.2016, leg. KI, KJ, PA, TB.

\section{Otiorhynchus (Choilisanus) pelliceus BOHEMAN, 1842 \\ Published data: Negru and Roşca 1967, Raduta 2014}

\section{Otiorhynchus (Choilisanus) raucus (FABRICIUS, 1777) \\ Published data: FleCK 1905, MontANDON 1908}

New data: Tulcea County: Celic Dere, 22-24. 05. 1997, leg. RS, CH, (Coll. Mus. G. ANTIPA) - Codru Monastery, 23-25.V.2013, leg. KI, PA, RI, SzL, 24.VI.2017, leg. KI, KJ, PA, TB; Slava Rusă, 24 25.V.2013, leg. KI, PA, RI, SzL, 1-3.V.2014, leg. KI, IZ, PA, SzL, 20.V.2015, leg. PA, RI, SzK, SzL, 31.III.2016, leg. FK, KI, PA, SzL, 9.V.2017, leg. FK, KI, KJ, PA, 11.V.2017, leg. FK, KI, KJ, PA; Babadag (forest), 30.IV.2014, leg. KI, IZ, PA, SzL, 24.VI.2017, leg. KI, KJ, PA, TB; Slava Rusă, Uspenia Monastery, 11.V.2017, leg. FK, KI, KJ, PA; 2,7 km from Horia to S, 28.VI.2017, leg. KI, KJ, PA, TB; 2 km from Slava Rusă to NE, 3.VI.2018, leg. FK, KI, KJ, PA; Constanţa County: Băneasa, Canaraua Fetii (quarry), 2-3.IV.2016, leg. FK, KI, PA, SzL; Băneasa, Canaraua Fetii (forest) 15-17.IX.2016, leg. TB;

Otiorhynchus (Choilisanus) velutinus GERMAR, 1823 Published data: MONTANDON 1887, 1908; JAQUET 1898; Fleck 1905; Negru and RoşCa 1967; RaduTA 2014

New data: Tulcea County: Enisala, 24-25.V.2013, leg. KI, PA, RI, SzL; Codru Monastery, 19.V.2015, leg. PA, RI, SzK, SzL; Constanţa County: Rezervaţia Naturală Agigea, 17.VII.2003, leg. AP (Coll. Mus. G. ANTIPA) - Murfatlar, Fântâniţa, 4-5.VII.2014, leg. KI, OA, PA, SzL, 22.V.2015, leg. PA, RI, SzK, SzL, 24-25.IX.2015, leg. KI, KJ, PA, 14.IX.2016, leg. KI, KJ, PA, TB; Sinoie, Grindul Lupilor, 5.VI.2018, leg. FK, KI, KJ, PA.

Otiorhynchus (Crataegodes) crataegi GERMAR, 1823 New data: Tulcea County: Babadag (forest), 30.IV.2014, leg. KI, IZ, PA, SzL, 13.IX.2016, leg. KI, KJ, PA, TB; Slava Rusă, 1-3.V.2014, leg. KI, IZ, PA, SzL; 2,5 km from Stejaru to NW, 25.VI.2017, leg. KI, KJ, PA, TB; Constanţa County: Pădurea Hagieni (forest), 6.VII.2014, leg. KI, OA, PA, SzL; Băneasa, Canaraua Fetii (forest), 15-17.IX.2016, leg. KI, KJ, PA, TB; Esechioi, Lacul Bugeac, towards the south Lake Bugeac, 16.IX.2016, leg. KI, KJ, PA, TB; Cobadin, 14.V.2017, leg. FK, KI, KJ, PA.
Otiorhynchus (Cryphiphorus) ligustici (LINNÉ, 1758) Published data: FleCK 1905, MONTANDON 1908, RADUTA 2014

New data: Tulcea County: Babadag, Codru, 25.V.1975, leg. NS; Măcin, leg. ALM; Pădurea Babadag, 20.VI.1963, leg. XS; Celic Dere, 22 24.V.1997, leg. RS, CH; Luncaviţa,Valea Fagilor, 23.V.1997, leg. RS (Coll. Mus. G. ANTIPA) - Iancina, 11.VI.1993, leg. TLA; Agighiol, 12.VI.1993, leg. TLA; Babadag (forest), 30.IV.2014, leg. KI, IZ, PA, SzL; Slava Rusă, 1-3.V.2014, leg. KI, IZ, PA, SzL, 9.V.2017, leg. KI; Constanţa County: Mangalia, leg. ALM; Murfatlar, 6.V.1951, leg. NS; Dumbrăveni, 23.V.1965, leg. NS; Pădurea Hagieni, 6.VI.1965, leg. XS, 20.VII.1997, leg. MiS; Esechioi, 10-14.V.1963, leg. NS, 30.V.1981, leg. NS, 2-5.V.1981, leg. NS, 26-29.V.1982, leg. NS; Agigea, 15.VI.1964, leg. XS; Băneasa, 9.VI.1981, leg. NS, 30.V.1982, leg. NS; Limanu, Pădurea Hagieni, 11.VII.1992, leg. RS; Băneasa, Canaraua Fetii, 18.V.1993, leg. CP, 23.VII.1993, leg. GA, 19.V.1994, leg. GA; Negureni, Pădurea Negureni, 18.V.1993, leg. CP; Hagieni, 22.V.1993, leg. CH; Furnica, Rezervaţia Dumbrăveni, 13-14.V.1995, leg. CP, 12-14.V.1995, leg. GA, MA; Canton Silvic Furnica, Pădurea Dumbrăveni, 13.V.1995, leg. CP (Coll. Mus. G. AnTIPA) - Gura Dobrogei, 28-29.IV.2012, leg. KI, 26.V.2013, leg. KI, PA, RI, SzL, 30.IV-2.V.2014, leg. KI, IZ, PA, SzL, 7.VI.2018, leg. FK, KI, KJ, PA; Cheile Dobrogei, 2.V.2014, leg. KI, IZ, PA, SzL; Negureni, Pădurea Valea Cişmelelor, 23.V.2015, leg. PA, RI, SzK, SzL; Băneasa, Canaraua Fetii (quarry), 2-3.IV.2016, leg. FK, KI, PA, SzL; Murfatlar, Fântâniţa, 7-8.VI.2018, leg. FK, KI, KJ, PA.

\section{Otiorhynchus (Otiorhynchus) multipunctatus} (FABRICIUS, 1792)

Published data: Montandon 1887, 1908; JAQUET 1900b; FLECK 1905

New data: Tulcea County: M-ţii Măcin-Ţuţuiatu (450 m), 18.VI.2005, leg. AR (Coll. Mus. R. ANGHELUȚĂ) - M-ţii Măcin, Valea Fagilor, 30.V.2005, leg. CsS; Babadag (forest), 23-25.V.2013, leg. KI, PA, RI, SzL, 13.IX.2016, leg. KI, KJ, PA, TB; Slava Rusă, 24 25.V.2013, leg. KI, PA, RI, SzL, 23.IX.2015, leg. KI, KJ, PA, 9.V.2017, leg. KI; M-ţii Măcin, Nifon (forest), 23.VI.2017, leg. KI, KJ, PA, TB; Nifon, pârâul Pârlita valley, 23.VI.2017, leg. KI, KJ, PA, TB.

Otiorhynchus (Pendragon) merklii STIERLIN, 1880 Published data: Fleck 1905, NeGru and RoşCa 1967, RADUTA 2014

Otiorhynchus (Pendragon) ovatus ovatus (LINNÉ, 1758)

Published data: Fleck 1905, Teodor and Manole 1996

New data: Tulcea County: Caraorman, 19.VII.1960, leg. IS, 23.IX.1967, leg. ŞN (Coll. Mus. G. ANTIPA) 
- Pădurea Letea (forest), 17.VI.1993, leg. TLA; Babadag (forest), 23-25.V.2013, leg. KI, PA, RI, SzL; Constanţa County: Agigea, 1.VII.1977, leg. GA, 1.VII.1977, leg. APG, 4.VII.1977, leg. GA(Coll. Mus. G. ANTIPA) - Murfatlar, Fântâniţa, 4-5.VII.2014, leg. KI, OA, PA, SzL.

Otiorhynchus (Pliadonus) frater STIERLIN, 1861 Published data: MONTANDON 1908

New data: Tulcea County: Codru Monastery, 22.IX.2015, leg. KI, KJ, PA, 30-31.III.2016, leg. FK, KI, PA, SzL.

\section{Otiorhynchus (Podoropelmus) albidus STIERLIN, 1861}

New data: Tulcea County: M-ţii Măcin-Valea lui Arsu, 6.VI.2005, leg. MC (Coll. Mus. R. ANGHELuȚă) - Codru env., 3.V.2009, leg. JK; NE of Topolog, 4.V.2009, leg. JK; Babadag (forest), 23-25.V.2013, leg. KI, PA, RI, SzL, 22.IX.2015, leg. KI, KJ, PA, 13.IX.2016, leg. KI, KJ, PA, TB; Codru Monastery, 23-25.V.2013, leg. KI, PA, RI, SzL, 30.IV-3.V.2014, leg. KI, IZ, PA, SzL, 19.V.2015, leg. PA, RI, SzK, SzL, 22.IX.2015, leg. KI, KJ, PA, 30-31.III.2016, leg. FK, KI, PA, SzL, 13.IX.2016, leg. KI, KJ, PA, TB, 24.VI.2017, leg. KI, KJ, PA, TB, 20.VI.2019, leg. KI, KJ, PA, TB; Slava Rusă, 24-25.V.2013, leg. KI, PA, RI, SzL, 1-3.V.2014, leg. KI, IZ, PA, SzL, 23.IX.2015, leg. KI, KJ, PA, 31.III.2016, leg. FK, KI, PA, SzL, 9.V.2017, leg. FK, KI, KJ, PA; M-ţii Măcin, 5 km S Greci, 8.V.2017, leg. FK, KI, KJ, PA; Enisala (castle), 10.V.2017, leg. FK, KI, KJ, PA; 2,7 $\mathrm{km}$ from Horia to S, 28.VI.2017, leg. KI, KJ, PA, TB; $3 \mathrm{~km}$ from Slava Rusă to NE, 24.VI.2017, leg. KI; $2 \mathrm{~km}$ from Slava Rusă to NE, 3.VI.2018, leg. FK, KI, KJ, PA; Constanţa County: Murfatlar, X.1951, leg. NS (Coll. Mus. G. ANTIPA) - $3 \mathrm{~km} \mathrm{~W}$ of Crucea, Allah Bair (Nature Reserve), 1.V.2009, leg. JK; Gura Dobrogei, 28-29.IV.2012, leg. KI, 25-27.VI.2017, leg. KI; Murfatlar, Fântâniţa, 4-5.VII.2014, leg. KI, OA, PA, SzL, 22.V.2015, leg. PA, RI, SzK, SzL, 24-25.IX.2015, leg. KI, KJ, PA, 14.IX.2016, leg. KI, KJ, PA, TB, 7-8.VI.2018, leg. FK, KI, KJ, PA; Negru Vodă (Nature Reserve), 25.IX.2015, leg. KI, KJ, PA; Esechioi (forest), 1-2.IV.2016, leg. FK, KI, PA, SzL; Băneasa, Canaraua Fetii (forest), 15-17.IX.2016, leg. KI, KJ, PA, TB; Esechioi, Lacul Bugeac, towards the south Lake Bugeac, 16.IX.2016, leg. KI, KJ, PA, TB; Cobadin, 14.V.2017, leg. FK, KI, KJ, PA; NeptunOlimp, Pădurea Comorova, 17.IX.2016, leg. FK, KI, KJ, PA; Pecineaga (forest), 14.V.2017, leg. FK, KI, KJ, PA.

Otiorhynchus (Podoropelmus) cf. fullo (ScHRANK, 1781)

New data: Constanţa County: Esechioi, Lacul Bugeacului, towards the south Lake Bugeac, 16.IX.2016, leg. KI, KJ, PA, TB.
Otiorhynchus (Podoropelmus) fullo (SCHRANK, 1781) Published data: FLECK 1905

New data: Tulcea County: M-ţii Măcin, Valea Fagilor, 30.V.2005, leg. CsS; Babadag (forest), 23-25.V.2013, leg. KI, PA, RI, SzL, 30.IV.2014, leg. KI, IZ, PA, SzL, 13.IX.2016, leg. KI, KJ, PA, TB; Codru Monastery, 23-25.V.2013, leg. KI, PA, RI, SzL, 19.V.2015, leg.PA, RI, SzK, SzL; Slava Rusă, 24-25.V.2013, leg. KI, PA, RI, SzL, 1-3.V.2014, leg. KI, IZ, PA, SzL. 20.V.2015, leg. PA, RI, SzK, SzL, 23.IX.2015, leg. KI, KJ, PA, 9.V.2017, leg. FK, KI, KJ, PA; Caugagia, 1.V.2014, leg. KI, IZ, PA, SzL; NE of Topolog, 4.V.2009, leg. JK; M-ţii Măcin, Nifon (forest), 23.VI.2017, leg. TB; $3 \mathrm{~km}$ from Slava Rusă to NE, 24.VI.2017, leg. KI; Constanţa County: Furnica, Pădurea Dumbrăveni, 14.V.1995, leg. MA; Negureni, 25.VI.1995, leg. CP (Coll. Mus. G. ANTIPA) - Băneasa, Canaraua Fetii (forest), 23.V.2015, leg. PA, RI, SzK, SzL, 15-17. IX.2016, leg. KI, KJ, PA, TB.

Otiorhynchus (Podoropelmus) rotundus MARSEUL, 1872 (= smreczynskii CMOLUCH, 1968)

Published data: Bialooki 2007

New data: Tulcea County: Codru Monastery, 2325.V.2013, leg. KI, PA, RI, SzL, 19.V.2015, leg. PA, RI, SzK, SzL, 22.IX.2015, leg. KI, KJ, PA, 30-31. III.2016, leg. FK, KI, PA, SzL, 13.IX.2016, leg. KI, KJ, PA, TB, 24-25.VI.2017, leg. KI, KJ, PA, TB; Enisala (castle), 13.IX.2016, leg. TB; Constanţa County: $3 \mathrm{~km} \mathrm{~W}$ of Crucea, Allah Bair (Nature Reserve), 1.V.2009, leg. JK; Murfatlar, Fântâniţa, 4-5.VII.2014, leg. KI, OA, PA, SzL, 22.V.2015, leg. PA, RI, SzK, SzL, 7-8.VI.2018, leg. FK, KI, KJ, PA; Gura Dobrogei, 30.IV-2.V.2014, leg. KI, IZ, PA, SzL, 7.VI.2018, leg. KI.

[Otiorhynchus (Prilisvanus) opulentus GERMAR, 1836]

Published data: FLECK 1905 - Living in the Carpathians only. Incorrect identification.

Otiorhynchus (Prodeminus) maxillosus GyllenHAL, 1834

Published data: Jaquet 1898, Fleck 1905, Negru and RoşCa 1967, Raduta 2014

Otiorhynchus (Pseudocryphiphorus) chrysostictus Gyllenhal, 1834 (= conspersus (Herbst, 1795)

Published data: Montandon 1908

New data: Tulcea County: Babadag (forest), 13.IX.2016, leg. TB; Constanţa County: Murfatlar, Fântâniţa, 24-25.IX.2015, leg. KI, KJ, PA; Esechioi, 15.IX.2016, leg. KI, KJ, PA, TB.

*Otiorhynchus (Pseudocryphiphorus) zebei Stierlin, 1861 (fig. 20)

New data: Constanţa County: Murfatlar, Fântâniţa, 4-5.VII.2014, leg. KI, OA, PA, SzL, 24-25.IX.2015, leg. KI, KJ, PA, 14.IX.2016, leg. KI, KJ, PA, TB. 
Otiorhynchus (Zustalestus) rugosostriatus (GOEZE, 1777)

Published data: Montandon 1908, Negru and RoşCa 1967, RADUTA 2014

New data: Tulcea County: Periprava, 9.V.1964, leg. XS (Coll. Mus. G. ANTIPA) - Codru env., 5.V.2009, leg. JK; Babadag, (forest), 30.IV.2014, leg. KI, IZ, PA, SzL, 22.IX.2015, leg. KI, KJ, PA, 13.IX.2016, leg. TB; Codru Monastery, 19.V.2015, leg. PA, RI, SzK, SzL; M-ții Măcin, 5 km S Greci, 8.V.2017, leg. FK, KI, KJ, PA; M-ţii Măcin, Nifon (forest), 23.VI.2017, leg. KI, KJ, PA, TB; Constanţa County: Hagieni, 25.X.1965, leg. ŞN, 26-29.X.1965, leg. ID (Coll. Mus. G. ANTIPA) -Pădurea Hagieni (forest), 6.VII.2014, leg. KI, OA, PA, SzL; Murfatlar, Fântâniţa, 22.V.2015, leg. PA, RI, SzK, SzL, 14.IX.2016, leg. KI, KJ, PA, TB, 7-8. VI.2018, leg. FK, KI, KJ, PA; Negru Vodă (Nature Reserve) 25.IX.2015, leg. KI, KJ, PA; Esechioi, Lacul Bugeac, towards the south Lake Bugeac, 16.IX.2016, leg. KI, KJ, PA, TB.

Parameira (Stierlinia) setosa SeIDLITZ, 1868

Published data: MonTANDOn 1906, 1908; NEgru and RoşCa 1967; Magnano and Osella 1971; Osella and Magnano 1984; Raduta 2014

New data: Tulcea County: Enisala (castle), 13.IX.2016, leg. KI, KJ, PA, TB; Constanţa County: $3 \mathrm{~km} \mathrm{~W}$ of Crucea, Allah Bair (Nature Reserve), 1.V.2009, leg. JK, 18.IX.2010, leg. JK; Istria env., Cetatea Histria, 2010.IX.22, leg. JK.

\section{Stomodes gyrosicollis BoHEMAN, 1842}

New data: Tulcea County: Slava Rusă, 1-3.V.2014, leg. KI, IZ, PA, SzL, 31.III.2016, leg. FK, KI, PA, SzL; Constanţa County: Gura Dobrogei, 30.IV2.V.2014, leg. KI, IZ, PA, SzL, 12.V.2017, leg. FK, KI, KJ, PA, 7.VI.2018, leg. KI; Murfatlar, Fântâniţa, 4-5.VII.2014, leg. KI, OA, PA, SzL, 22.V.2015, leg. PA, RI, SzK, SzL, 7-8.VI.2018, leg. FK, KI, KJ, PA.

Stomodes puncticollis puncticollis TOURNIER, 1864

Published data: Osella and Magnano 1984

Stomodes rotundicollis J. FRIVALDSZKY, 1880

Published data: FLeCK 1905, Negru and RoşCa 1967, RADUTA 2014

Stomodes tolutarius SCHÖNHERR, 1826

Published data: Montandon 1908, Negru and RoşCa 1967, RADUTA 2014

Peritelini LaCordaIRE, 1863

Peritelus familiaris Boheman, 1834

Published data: JAQUET 1899a, 1899b, 1902b; FLECK 1905; Montandon 1908; Negru and RoşCa 1967; Negru 1968; Ieniştea 1968, 1974; TeOdor 1993; TeOdor and Manole 1996; Raduta 2014 New data: Tulcea County: C. A. Rosetti, Deltă, 12.V.1961, leg. MW, 12.V.1964, leg. MW, 8-9.

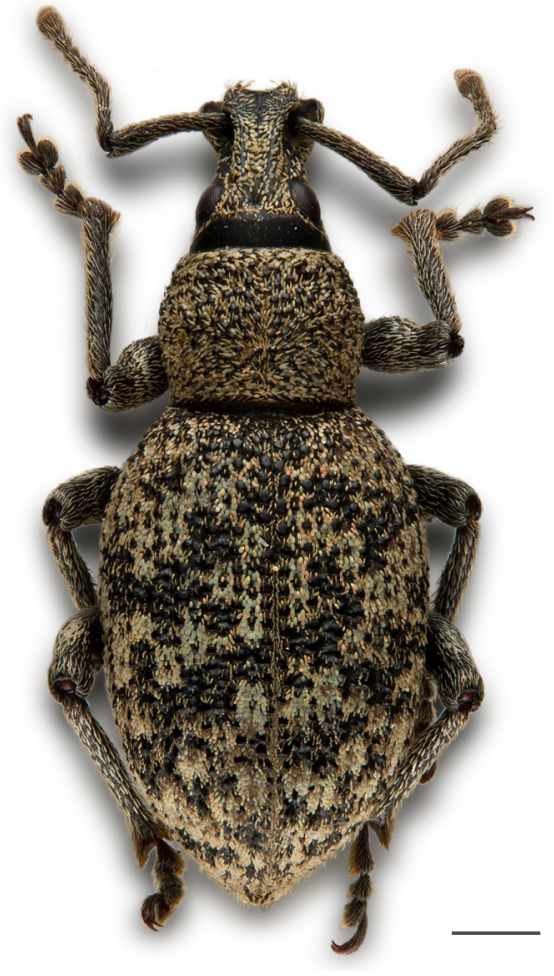

Fig. 20. Otiorhynchus (Pseudocryphiphorus) zebei StiERLin, 1861. Scale bar: $1 \mathrm{~mm}$. Photo Zoltán Csata

VI.1981, leg. CP; C. A. Rosetti, Pădurea Letea, 9.V.1964, leg. IS, 10.V.1964, leg. XS, MW, 11.V.1964, leg. MW, 11.V.1964, 12.V.1964, leg. XS, 09.V.1965, leg. IS, 5.VI.1979., 26.V.1980, leg. APG, 4.V.2012, leg. IŞI; Periprava, 9.V.1964, leg. MW, 13.V.1964, leg. XS, 16.IV.1966, leg. ID; Letea, 04.VII.1965; Caraorman, 6.V.1967, leg. MW, 9.V.1967, leg. XS, 12.V.1967, leg. MW, 17.VI.1967, leg. ŞN, 11.VI.1969, leg. MW, 15.VI.1969, leg. ŞN,

25.VI.1991, leg. NG, 26.VI.1991, leg. GA, 15.V.1992, leg. NG; Jurilovca, Pădurea 6 Martie, 10.V.1992, leg. NR; Sfântu Gheorghe, Delta Dunării, 14.V.1992; Haşmacul lui Omer, Delta Dunării, 19-20.VI.1993, leg. IM (Coll. Mus. G. AnTIPA) - Pădurea Letea (forest), 17.VI.1993, leg. TLA; Constanţa County: Agigea, 15.VI.1964, leg. XS, 22.VI.1964, leg. MW (Coll. Mus. G. Antipa).

[Peritelus sphaeroides GeRMAR, 1823]

Published data: Negru and RoşCa 1967, IEniştea 1974, Raduta 2014 - Spread: Western Europe. Incorrect identification.

[Pseudomeira rudis (BOHEMAN, 1842)]

Published data: FLECK 1906 - Living in Italy only. Incorrect identification.

Phyllobiini SCHÖNHERR, 1826

*Argoptochus (Argoptochus) markovensis ANGELOV, 1987 (fig. 21)

New data: Tulcea County: Babadag (forest), 2.VI.2005, leg. CsS; Slava Rusă, 24-25.V.2013, leg. KI, PA, RI, SzL, 20.V.2015, leg. PA, RI, SzK, SzL; 


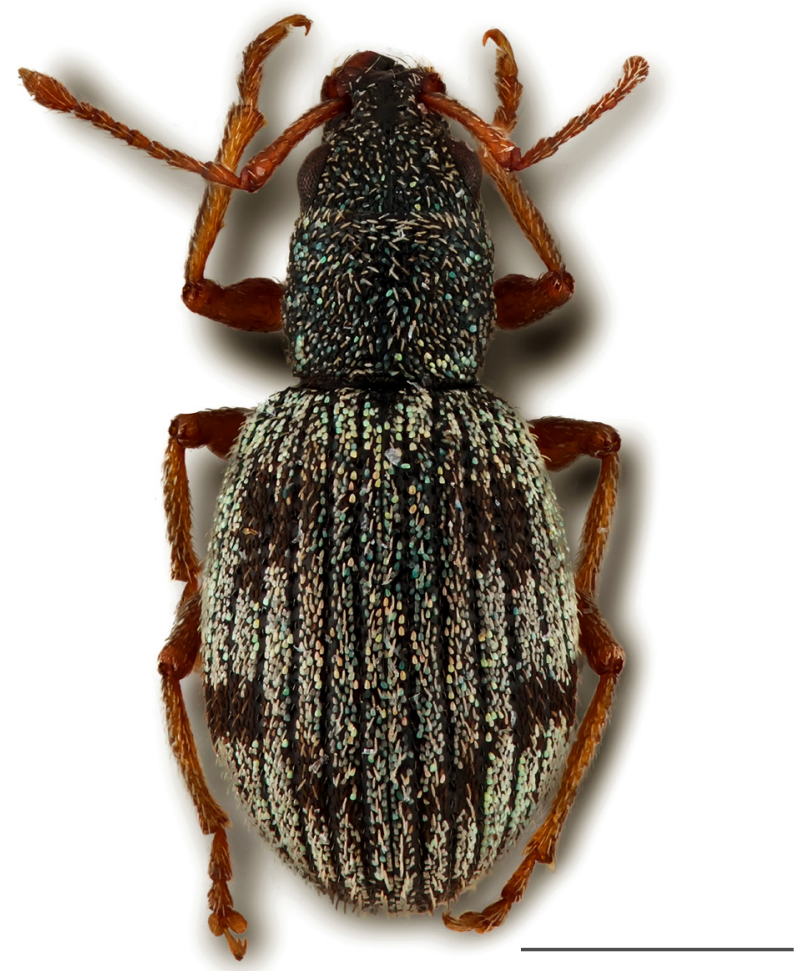

Fig. 21. Argoptochus (Argoptochus) markovensis ANGELOV, 1987 . Scale bar: $1 \mathrm{~mm}$. Photo Zoltán CsatA

M-ţii Măcin, 5 km S Greci, 8.V.2017, leg. FK, KI, KJ, PA, 18-19.VI.2019, leg. KI, KJ, PA, TB.

Oedecnemidius varius (BRULLÉ, 1832) (= Phyllobius pictus GYLLENHAL, 1834)

Published data: Montandon 1906, 1908; TeOdor 1993; RADUTA 2014

New data: Tulcea County: C. A. Rosetti, VII.1950, coll. IC; Babadag, Visterna, 12.IV.1960., 12.IV.1961, 15.IV.1963， 17.IV.1963, leg. IC; Celic Dere, 20.V.1997, leg. CH (Coll. Mus. G. Antipa) - Slava Rusă, 23.IV.2009, leg. TLA, 9.V.2017, leg. FK, KI, KJ, PA, 11.V.2017, leg. FK, KI, KJ, PA; Codru env., 3.V.2009, leg. JK; S of Horia, 6.V.2009, leg. JK; Babadag, (forest), 23-25.V.2013, leg. KI, PA, RI, SzL, 20.V.2017, leg. CS; Codru Monastery, 30.IV3.V.2014, leg. KI, IZ, PA, SzL, 10-11.V.2017, leg. FK, KI, KJ, PA; M-ţii Măcin, 5 km S Greci, 8.V.2017, leg. FK, KI, KJ, PA; Enisala (castle), 10.V.2017, leg. FK, KI, KJ, PA; M-ţii Măcin, Hamcearca, Culmea Cardonului, 19.V.2017, leg. CS; Constanţa County: Furnica, Rezervaţia Dumbrăveni, 13-14.V.1995, leg. CP (Coll. Mus. G. ANTIPA) - Negureni, Pădurea Valea Cişmelelor, 23.V.2015, leg. PA, RI, SzK, SzL; Pecineaga (forest), 14.V.2017, leg. FK, KI, KJ, PA; Limanu, 12.V.2017, leg. FK, KI, KJ, PA; NeptunOlimp, Pădurea Comorova, 17.IX.2016, leg. FK, KI, KJ, PA.

Phyllobius (Dieletus) argentatus argentatus (LINNÉ, 1758)

New data: Tulcea County: Babadag, Visterna, 12.IV.1961, coll. IC (Coll. Mus. G. ANTIPA) - Nifon, pârâul Pârlita valley, 23.VI.2017, leg. TB.

Phyllobius (Ectomogaster) fulvago GYLLENHAL, 1834 Published data: MonTANDON 1908

New data: Tulcea County: Cerna, 26.V.2005, leg. IM (Coll. Mus. G. Antipa).

\section{Phyllobius (Metaphyllobius) pomaceus GYLLENHAL,} 1834

New data: Tulcea County: Măcin, 10.V.2011, leg. IŞI (Coll. Mus. G. AntiPA); Constanţa County: Negureni, Pădurea Negureni, 18.V.1993, leg. CP, AP; Băneasa, Canaraua Fetii, 19.V.1993, leg. MB, CH, 20.V.1993, leg. CP, 19.V.1994, leg. MA; Canton Silvic Furnica, Pădurea Dumbrăveni, 13.V.1995, leg. CP; Furnica, Rezervaţia Dumbrăveni, 13-14.V.1995, leg. CP; Furnica, Pădurea Dumbrăveni, 12.V.1995, leg. CP, 13-14.V.1995, leg. CP, 22.VI.1995, leg. CP (Coll. Mus. G. ANTIPA) - Negureni, Pădurea Valea Cişmelelor, 23.V.2015, leg. PA, RI, SzK, SzL.

\section{Phyllobius (Nemoicus) oblongus (LINNÉ, 1758)}

New data: Tulcea County: Luncaviţa, Valea Fagilor, 23.V.1997, leg. RS (Coll. Mus. G. ANTIPA) - M-ţii Măcin, Valea Fagilor, 30.V.2005, leg. CsS; Atmagea, 28.IV.2012, leg. KI; Babadag (forest), 23-25.V.2013, leg. KI, PA, RI, SzL, 30.IV.2014, leg. KI, IZ, PA, SzL; Slava Rusă, 24-25.V.2013, leg. KI, PA, RI, SzL, 1-3.V.2014, leg. KI, IZ, PA, SzL, 20.V.2015, leg. PA, RI, SzK, SzL, 9.V.2017, leg. FK, KI, KJ, PA, 11.V.2017, leg. FK, KI, KJ, PA; Caugagia, 1.V.2014, leg. KI, IZ, PA, SzL; Slava Rusă, Uspenia Monastery, 11.V.2017, leg. FK, KI, KJ, PA; Constanţa County: Băneasa, Canaraua Fetii, 20.V.1993, leg. AP (Coll. Mus. G. ANTIPA) - Murfatlar, Fântâniţa, 22.V.2015, leg. PA, RI, SzK, SzL; Băneasa, Canaraua Fetii, 23.V.2015, leg. PA, RI, SzK, SzL, 2-3.IV.2016, leg. FK, KI, PA, SzL; Bugeac, 2.IV.2016, leg. FK, KI, PA, SzL; Ostrov, 2.IV.2016, leg. FK, KI, PA, SzL; Esechioi (forest), 1-2.IV.2016, leg. FK, KI, PA, SzL; Albeşti, Pădurea Hagieni (forest), 12.V.2017, leg. FK, KI, KJ, PA; Hagieni, Pădurea Hagieni (forest), 17.IX.2016, leg. FK, KI, KJ, PA; Pecineaga (forest), 14.V.2017, leg. FK, KI, KJ, PA.

\section{Phyllobius (Phyllobius) betulinus betulinus} (BECHSTEIN \& SCHARFENBERG, 1805)

New data: Constanţa County: Mangalia, Pădurea Comorova, 30.V.1963, leg. XS (Coll. Mus. G. Antipa)Murfatlar, Fântâniţa, 22.V.2015, leg. PA, RI, SzK, SzL, 7-8.VI.2018, leg. FK, KI, KJ, PA; Limanu, 12.V.2017, leg. FK, KI, KJ, PA; Hagieni, Pădurea Hagieni (forest), 17.IX.2016, leg. FK, KI, KJ, PA; Albeşti, Pădurea Hagieni (forest), 12.V.2017, leg. FK, KI, KJ, PA.

\section{Phyllobius (Phyllobius) pyri (LINNÉ, 1758)}

New data: Tulcea County: M-ţii Măcin, Pricopan (300 m), 21.VI.2004, leg. AR (Coll. Mus. R. ANGHeluȚĂ) - Babadag, Valea Caugagie, 17.V.1963, 
leg. IC; Chilia Veche, Deltă, 7.V.1964, leg. XS; Celic Dere, 24.V.1997, leg. RS (Coll. Mus. G. AnTIPA) Atmagea, 23.IV.2009, leg. TLA, 26.IV.2012, leg. KI; Horia-Praporgescu, 23.IV.2009, leg. TLA; Măcin, Rezervaţia de la Greci, 23.IV.2009, leg. TLA; Slava Rusă, 1-3.V.2014, leg. KI, IZ, PA, SzL, 9.V.2017, leg. FK, KI, KJ, PA; Smârdan, Braţul Măcin, 7.V.2017, leg. FK, KI, KJ, PA; Enisala (castle), 10.V.2017, leg. FK, KI, KJ, PA; Constanţa County: Cochirleni, 24.V.2015, leg. PA, RI, SzK, SzL, 3. IV.2016, leg. FK, KI, PA, SzL; Băneasa, Canaraua Fetii (quarry), 2-3. IV.2016, leg. FK, KI, PA, SzL; Ostrov, 2.IV.2016, leg. FK, KI, PA, SzL.

\section{Phyllobius (Phyllobius) seladonius BRULLÉ, 1832}

New data: Tulcea County: M-ţii Măcin, Plopişor, 15.VI.2005, leg. MC (Coll. Mus. R. Angheluță); Pădurea Ciucurova, 22.V.1997, leg. RS; Luncaviţa, Valea Fagilor, 23.V.1997, leg. CP; Luncaviţa, Parcul Naţonal M-ţii Măcin, 24.V.2005, leg. MS; Niculiţel, Mănăstirea Cocoş, 24.V.2005, leg. IM; Cerna, 26.V.2005, leg. IM (Coll. Mus. G. ANTIPA) - M-ţii Măcin, Valea Fagilor, 30.V.2005, leg. CsS; Frecăţei, Celic Dere Monastery (hillside) 31.V.2005, leg. CsS; Babadag, (forest), 2.VI.2005, leg. CsS; Podisul Babadagului, 2007.V.17, leg. NJ, RE, SoL, VJ; Măcin, Rezervaţia de la Greci, 23.IV.2009, leg. TLA; S of Horia, 6.V.2009, leg. JK; M-ţii Măcin, Greci env., 6.V.2009, leg. JK; NE of Topolog, 4.V.2009, leg. JK; Slava Rusă, 23.IV.2009, leg. TLA, 24-25.V.2013, leg. KI, PA, RI, SzL, 1-3.V.2014, leg. KI, IZ, PA, SzL, 20.V.2015, leg. PA, RI, SzK, SzL, 9.V.2017, leg. FK, KI, KJ, PA, 11.V.2017, leg. FK, KI, KJ, PA; Babadag, (forest), 30.IV.2014, leg. KI, IZ, PA, SzL; Caugagia, 1.V.2014, leg. KI, IZ, PA, SzL; M-ţii Măcin, 5 km S Greci, 8.V.2017, leg. FK, KI, KJ, PA; Codru Monastery, 10-11.V.2017, leg. FK, KI, KJ, PA; Enisala (castle), 10.V.2017, leg. FK, KI, KJ, PA; 2,5 $\mathrm{km}$ from Stejaru to NW, 25.VI.2017, leg. KI, KJ, PA, TB; 2 km from Slava Rusă to NE, 3.VI.2018, leg. FK, KI, KJ, PA; Constanţa County: Murfatlar, Fântâniţa, 22.V.2015, leg. PA, RI, SzK, SL; Băneasa, Canaraua Fetii, 23.V.2015, leg. PA, RI, SzK, SzL; Negureni, Pădurea Valea Cişmelelor, 23.V.2015, leg. PA, RI, SzK, SzL; Negru Vodă (Nature Reserve) 25.IX.2015, leg. KI, KJ, PA; Albeşti, Pădurea Hagieni (forest), 12.V.2017, leg. FK, KI, KJ, PA.

Phyllobius (Phyllobius) thalassinus GYLLENHAL, 1834 Published data: MonTANDON 1908

New data: Tulcea County: M-ţii Măcin, Greci, 7.V.2017, leg. FK, KI, KJ, PA; M-ţii Măcin, 5 km S Greci, 8.V.2017, leg. FK, KI, KJ, PA; Slava Rusă, 9.V.2017, leg. FK, KI, KJ, PA, 11.V.2017, leg. FK, KI, KJ, PA.

Phyllobius (Plagius) pallidus (FABRICIUS, 1792)

(= incanus GyLlenHAL, 1834)

New data: Tulcea County: Babadag, Visterna,
16.V.1963, leg. IC (Coll. Mus. G. ANTIPA) - Codru env., 3.V.2009, leg. JK; Măcin, Rezervaţia de la Greci, 23.IV.2009, leg. TLA; Slava Rusă, 23.IV.2009, leg. TLA, 1-3.V.2014, leg. KI, IZ, PA, SzL, 9.V.2017, leg. FK, KI, KJ, PA; S of Horia, 6.V.2009, leg. JK; Slava Rusă, Uspenia Monastery, 11.V.2017, leg. FK, KI, KJ, PA.

\section{Phyllobius (Pterygorrhynchus) contemptus SCHÖNHERR, 1832 \\ New data: Constanța County: Murfatlar, Fântâniţa, 22.V.2015, leg. PA, RI, SzK, SzL.}

\section{Pseudomyllocerus sinuatus (FABRICIUS, 1801)}

Published data: Montandon 1887, Fleck 1905, Negru and RoşCa 1967, Raduta 2014

New data: Tulcea County: Codru Monastery, 4.VI.2018, leg. FK, KI, KJ, PA; Constanţa County: Pădurea Hagieni (forest), 6.VII.2014, leg. KI, OA, PA, SzL; Gura Dobrogei, 25.VI.2017, leg. TB, 7.VI.2018, leg. FK, KI, KJ, PA; Murfatlar, Fântâniţa, 7-8.VI.2018, leg. FK, KI, KJ, PA.

Polydrusini SCHÖNHERR, 1823

Liophloeus (Liophloeodes) lentus GERMAR, 1823

Published data: MonTANDON 1906

Liophloeus (Liophloeus) tessulatus (O. F. MÜLLER, 1776)

Published data: FLECK 1905

New data: Tulcea County: Celic Dere, 20.V.1997, leg. RS (Coll. Mus. G. ANTIPA) - M-ţii Măcin, Valea Fagilor, 30.V.2005, leg. CsS; Slava Rusă, 9.V.2017, leg. FK, KI, KJ, PA.

\section{Polydrusus (Chlorodrosus) amoenus (GERMAR, 1823)}

New data: Tulcea County: C. A. Rosetti, VII.1950, leg. IC (Coll. Mus. G. ANTIPA); Constanţa County: Băneasa, Canaraua Fetii, 30.IX.1965, leg. ŞN (Coll. Mus. G. AntIPA).

*Polydrusus (Conocetus) baudii (FAUST, 1889)

New data: Constanţa County: Esechioi (forest), 7.VII.2014, leg. PA.

Polydrusus (Eudipnus) formosus (MAYER, 1779) (= sericeus SCHALLER, 1783; = thalassinus GYLLENHAL, 1834)

Published data: Montandon 1887, FleCK 1905, MonTANDON 1908

New data: Tulcea County: Măcin, Greci, leg. ALM; C. A. Rosetti, VII.1950., leg. IC; Babadag, Visterna, 12.IV.1961, 16.V.1963, leg. IC; Niculiţel, Mănăstirea Cocoş, 24.V.2005, leg. IM (Coll. Mus. G. AnTIPA) - M-ţii Măcin, Valea Fagilor, 30.V.2005, leg. CsS; M-ţii Măcin, Luncaviţa, 30.V.2005, leg. CsS; Babadag, (forest), 23-25.V.2013, leg. KI, PA, RI, SzL; Slava Rusă, 24-25.V.2013, leg. KI, PA, RI, SzL, 
1-3.V.2014, leg. KI, IZ, PA, SzL, 20.V.2015, leg. PA, RI, SzK, SzL, 11.V.2017, leg. FK, KI, KJ, PA; Nifon, pârâul Pârlita valley, 23.VI.2017, leg. KI, KJ, PA, TB; Constanţa County: Băneasa, 30.V.1965, leg. MW; Băneasa, Canaraua Fetii, 30.V.1965, leg. ŞN; Furnica, Rezervaţia Dumbrăveni, 13-14.V.1995, leg. CP (Coll. Mus. G. ANTIPA) - Murfatlar, Fântâniţa, 22.V.2015, leg. PA, RI, SzK, SzL; Băneasa, Canaraua Fetii, 23.V.2015, leg. PA, RI, SzK, SzL; Gura Dobrogei, 25-27.VI.2017, leg. KI, KJ, PA, TB.

\section{Polydrusus (Eudipnus) mollis (STRøм, 1768)}

New data: Tulcea County: Babadag, Visterna, 12.IV.1961, leg. IC (Coll. Mus. G. ANTIPA); Constanţa County: Cheile Dobrogei, 18.IV.2014, leg. CS.

\section{Polydrusus (Eurodrusus) pilosus pilosus GREDLER, 1866 \\ New data: Tulcea County: Codru Monastery, 10- 11.V.2017, leg. KI.}

\section{Polydrusus (Eustolus) corruscus GERMAR, 1823}

New data: Tulcea County: C. A. Rosetti, Delta Dunării, 9.VI.1981, leg. CP; Caraorman, 26.VI.1991, leg. IP; Valea Teilor, 22.V.1997, leg. CP (Coll. Mus. G. ANTIPA) - Braţul Sfântu Gheorghe, 23.VI.2003, leg. TB; NE of Topolog, 4.V.2009, leg. JK; Slava Rusă, 20.V.2015, leg. PA, RI, SzK, SzL; Smârdan, Braţul Măcin, 7.V.2017, leg. FK, KI, KJ, PA, 22.VI.2017, leg. KI, KJ, PA, TB, 2.VI.2018, leg. FK, KI, KJ, PA; Nifon, pârâul Pârlita valley, 23.VI.2017, leg. TB.

\section{Polydrusus (Eustolus) flavipes flavipes (DEGEER, 1775)}

Published data: TeOdor 1993, RAduta 2014

Polydrusus (Eustolus) impressifrons GYLLENHAL, 1834 Published data: TeOdor and Manole 1996; Raduta 2014

Polydrusus (Eustolus) pterygomalis BOHEMAN, 1840 New data: Tulcea County: Canalul Magearu, 17.VI.1993, leg. TLA; Gorgova, 16.VI.1993, leg. TLA; Slava Rusă, 24-25.V.2013, leg. KI, PA, RI, SzL, 1-3.V.2014, leg. KI, IZ, PA, SzL, 20.V.2015, leg. PA, RI, SzK, SzL, 11.V.2017, leg. FK, KI, KJ, PA; Babadag (forest), 30.IV.2014, leg. KI, IZ, PA, SzL; Slava Rusă, Uspenia Monastery, 11.V.2017, leg. FK, KI, KJ, PA; Enisala (castle), 10.V.2017, leg. FK, KI, KJ, PA; M-ţii Măcin, Nifon (forest), 23.VI.2017, leg. KI, KJ, PA, TB; 2 km from Slava Rusă to NE, 3.VI.2018, leg. FK, KI, KJ, PA.

Polydrusus (Poecilodrusus) viridicinctus
Gyllenhal, 1834
New data: Tulcea County: C. A. Rosetti, VII.1950,
leg. IC (Coll. Mus. G. ANTIPA) - Babadag (forest),
2005.VI.2, leg. CsS, 23-25.V.2013, leg. KI, PA, RI,
SzL; Constanţa County: Gura Dobrogei, 26.V.2013,

leg. KI, PA, RI, SzL.

Polydrusus (Polydrusus) ferrugineus BoHEMAN, 1840 (= Metadrosus ornatus GyLlenHAL, 1834)

New data: Tulcea County: Babadag, Visterna, 1416.V.1963, leg. IC, (Coll. Mus. G. ANTIPA) - Codru env., 3.V.2009, leg. JK; Slava Rusă, 1-3.V.2014, leg. KI, IZ, PA, SzL, 20.V.2015, leg. PA, RI, SzK, SzL, 9.V.2017, leg. FK, KI, KJ, PA; Codru Monastery, 1011.V.2017, leg. FK, KI, KJ, PA; Constanţa County: Esechioi (forest), 1-2.IV.2016, leg. FK, KI, PA, SzL; Băneasa, Canaraua Fetii (quarry), 2-3.IV.2016, leg. FK, KI, PA. SzL.

\section{Polydrusus (Polydrusus) picus picus (FABRICIUS, 1792)}

Published data: Montandon 1887, 1908; FLECK 1905; TEODOR 1993

New data: Tulcea County: C. A. Rosetti,VII.1950., leg. IC; Babadag, Visterna, 14-15.V.1963, leg. IC; Cerna, 26.V.2005, leg. CB; Măcin, Valea Suluc, 27.V.2005, leg. IM (Coll. Mus. G. Antipa) - Pădurea Letea (forest), 17.VI.1993, leg. TLA; Slava Rusă, 23.IV.2009, leg. TLA, 24-25.V.2013, leg. KI, PA, RI, SzL, 1-3.V.2014, leg. KI, IZ, PA, SzL, 20.V.2015, leg. PA, RI, SzK, SzL, 9.V.2017, leg. FK, KI, KJ, PA, 11.V.2017, leg. FK, KI, KJ, PA; Ciucurova env., 4.V.2009, leg. JK; Babadag (forest), 2.VI.2005, leg. CsS, 23-25.V.2013, leg. KI, PA, RI, SzL, 20.V.2017, leg. CS; Enisala, 29.IV-3.V.2014, leg. KI; Codru Monastery, 30.IV-3.V.2014, leg. KI, IZ, PA, SzL, 1011.V.2017, leg. FK, KI, KJ, PA; M-ţii Măcin, 5 km S Greci, 8.V.2017, leg. FK, KI, KJ, PA; Enisala (castle), 10.V.2017, leg. FK, KI, KJ, PA; Constanţa County: $3 \mathrm{~km} \mathrm{~W}$ of Crucea, Allah Bair (Nature Reserve), 1.V.2009, leg. JK; Gura Dobrogei, 26.V.2013, leg. KI, PA, RI, SzL, 30.IV-2.V.2014, leg. KI, IZ, PA, SzL, 25.VI.2017, leg. KI, KJ, PA, TB; Murfatlar, Fântâniţa, 4-5.VII.2014, leg. KI, OA, PA, SzL, 22.V.2015, leg. PA, RI, SzK, SzL, 7-8.VI.2018, leg. KI; Băneasa, Canaraua Fetii, 23.V.2015, leg. PA, RI, SzK, SzL.

Polydrusus (Scythodrosus) inustus Germar, 1823 Published data: FLECK 1905

New data: Tulcea County: Dinogeţia, 23.V.2002, leg. GP (Coll. Mus. R. Angheluță); Greci, 26.V.1961, leg. XS; Chilia Veche, Deltă, 07.V.1964, leg. XS, 7.V.1964, leg. MW; Celic Dere, 20.V.1997, leg. CH, 27.V.2005, leg. RS; Izvoarele, 21.V.1997, leg. RS; Ciucurova, 22.V.1997, leg. CH, 22.V.1997, leg. CP; Greci, Parcul Naţional M-ţii Măcinului, 25.V.2005, leg. CB (Coll. Mus. G. ANTIPA) - Măcin (hillside, 200 m), 29.V.2005, leg. CsS; M-ţii Măcin, Valea Fagilor (quarry) 30.V.2005, leg. CsS; Frecăței, Celic Dere Monastery (hillside) 31.V.2005, leg. CsS; M-ţii Măcin, Pricopan, 1.VI.2005, leg. CsS; Babadag (forest), 23-25.V.2013, leg. KI, PA, RI, SzL, 20.V.2017, leg. CS; Codru Monastery, 23-25.V.2013, leg. KI, PA, RI, SzL, 30.IV-3.V.2014, leg. KI, IZ, PA, SzL, 19.V.2015, 
leg. PA, RI, SzK, SzL, 10-11.V.2017, leg. FK, KI, KJ, PA, 24.VI.2017, leg. KI, KJ, PA, TB, 4.VI.2018, leg. FK, KI, KJ, PA, 20.VI.2019, leg. KI; Slava Rusă, 24-25.V.2013, leg. KI, PA, RI, SzL, 1-3.V.2014, leg. KI, IZ, PA, SzL, 20.V.2015, leg. PA, RI, SzK, SzL, 9.V.2017, leg. FK, KI, KJ, PA, 11.V.2017, leg. FK, KI, KJ, PA; Enisala, 29.IV-3.V.2014, leg. KI, IZ, PA, SzL; Smârdan, Braţul Măcin, 7.V.2017, leg. FK, KI, KJ, PA, 22.VI.2017, leg. KI, KJ, PA, TB, 2.VI.2018, leg. FK, KI, KJ, PA; Enisala (castle), 10.V.2017, leg. FK, KI, KJ, PA, 25.VI.2017, leg. KI; Nifon, pârâul Pârlita valley, 23.VI.2017, leg. KI, KJ, PA, TB; N from Nifon, 23.VI.2017, leg. KI, KJ, PA, TB; 1,5 km from Beidaud to SW, 25.VI.2017, leg. KI, KJ, PA, TB; S from Hamcearca, 24.VI.2017, leg. KI, KJ, PA, TB; Greci, 7-8.V.2017, leg. KI; M-ţii Măcin, 5 km S Greci, 8.V.2017, leg. KI; 2,7 km from Horia to S, 28.VI.2017, leg. KI, KJ, PA, TB; $2 \mathrm{~km}$ from Slava Rusă to NE, 3.VI.2018, leg. FK, KI, KJ, PA; Horea, 3.VI.2018, leg. PA; M-ţii Măcin, Greci, 18-19. VI.2019, leg. KI, KJ, PA, TB; Turcoaia, $6 \mathrm{~km}$ from Cerna to NW, 19.VI.2019, leg. KI, KJ, PA, TB; Constanţa County: Constanţa, 23.VI.1962, leg. AtR; Furnica, Rezervaţia Dumbrăveni, 13-14.V.1995, leg. CP (Coll. Mus. G. ANTIPA) - Gura Dobrogei, 28-29. IV.2012, leg. KI, 26.V.2013, leg. KI, PA, RI, SzL, 30.IV-2.V.2014, leg. KI, IZ, PA, SzL, 12.V.2017, leg. FK, KI, KJ, PA, 25-27.VI.2017, leg. KI, KJ, PA, TB; Murfatlar, Fântâniţa, 4-5.VII.2014, leg. KI, OA, PA, SzL, 22.V.2015, leg. PA, RI, SzK, SzL, 7-8.VI.2018, leg. FK, KI, KJ, PA; Pădurea Hagieni (forest), 6.VII.2014, leg. KI, OA, PA, SzL, 12.V.2017, leg. FK, KI, KJ, PA; Esechioi (forest), 7.VII.2014, leg. KI, OA, PA, SzL; Palazu Mic, 22.V.2015, leg. PA, RI, SzK, SzL; Băneasa, Canaraua Fetii, 23.V.2015, leg. PA, RI, SzK, SzL; Limanu, 12.V.2017, leg. FK, KI, KJ, PA; Hagieni, Pădurea Hagieni (forest), 17.IX.2016, leg. FK, KI, KJ, PA; 23 August, 17.IX.2016, leg. FK, KI, KJ, PA; Pecineaga (forest), 14.V.2017, leg. FK, KI, $\mathrm{KJ}, \mathrm{PA}$.

Polydrusus (Scythodrusus) piliferus НосннUтн, 1847 (= prasinus ReITTER, 1899)

Published data: FLECK 1905

Psallidiini LACORDAIRE, 1863

Mesagroicus obscurus BOHEMAN, 1840

Published data: Montandon 1908, Negru and RoşCa 1967, TEOdOR 1993, RAduTA 2014

New data: Tulcea County: Măcin, leg. ALM; Mănăstirea Cocoş, 08.V.1973, leg. MW (Coll. Mus. G. AntiPA) - Frecăţei, Celic Dere Monastery (hillside) 31.V.2005, leg. CsS; Atmagea, 28.IV.2012, leg. KI; Slava Rusă, 24-25.V.2013, leg. KI, PA, RI, SzL, 1-3.V.2014, leg. KI, IZ, PA, SzL; Nifon, pârâul Pârlita valley, 23.VI.2017, leg. KI, KJ, PA, TB; Smârdan, Braţul Măcin, 22.VI.2017, leg. KI; Constanţa County: Mangalia, leg. ALM; Furnica, Rezervaţia Dumbrăveni, 13-14.V.1995, leg. CP (Coll.
Mus. G. AntiPA) - Gura Dobrogei, 26.V.2013, leg. KI, PA, RI, SzL; Esechioi (forest), 7.VII.2014, leg. KI, OA, PA, SzL; Cochirleni, 3.IV.2016, leg. FK, KI, PA, SzL.

Psallidium maxillosum maxillosum (FABRICIUS, 1792) Published data: JAQUET 1900b; FLECK 1905; Montandon 1906, 1908; Negru and RoşCa 1967; RADUTA 2014

New data: Tulcea County: M-ţii Măcin, Luncaviţa (200 m), 16.VI.2005, leg. MC (Coll. Mus. R. ANGHeluță); Cerna, Parcul Naţional M-ţii Măcin, 26.V.2005, leg. MS; Măcin, leg. ALM; Babadag, 29.VI.1956, leg. XS (Coll. Mus. G. Antipa) - Beştepe, 14.VI.1993, leg. TLA; Iancina, 11.VI.1993, leg. TLA; Braţul Sfântu Gheorghe, 21-22.VI.2003, leg. TB; M-ţii Măcin, Pricopan, 1.VI.2005, leg. CsS; Atmagea, 23.IV.2009, leg. TLA; Codru Monastery, 23-25.V.2013, leg. KI, PA, RI, SzL; Enisala, 24-25.V.2013, leg. KI, PA, RI, SzL, 29.IV-3.V.2014, leg. KI, IZ, PA, SzL, 30-31. III.2016, leg. FK, KI, PA, SzL; M-ţii Măcin, 5 km S Greci, 8.V.2017, leg. FK, KI, KJ, PA; Enisala (castle), 10.V.2017, leg. FK, KI, KJ, PA; 2 km from Slava Rusă to NE, 3.VI.2018, leg. FK, KI, KJ, PA; Constanţa County: Agigea, 18.VI.1962, leg. XS, AtR; Constanţa, Palas, 21.VI.1962, leg. AtR; Valul lui Traian, 22.VI.1962, leg. AtR; Canaraua Fetii, 27.IV.1963, leg. NS; Furnica, Pădurea Dumbrăveni, 12.V.1995, leg. GA (Coll. Mus. G. ANTIPA) - $3 \mathrm{~km}$ W of Crucea, Allah Bair (Nature Reserve), 1.V.2009, leg. JK; Gura Dobrogei, 12.V.2017, leg. KI, 7.VI.2018, leg. KI.

\section{Sciaphilini SHARP, 1891}

\section{Archeophloeus inermis (BOHEMAN, 1842)}

(= Trachyphloeus inermis BOHEMAN, 1842)

Published data: Montandon 1908, Negru and RoşCa 1967, RADUTA 2014

New data: Tulcea County: Codru env., 3.V.2009, leg. JK; Codru Monastery, 10-11.V.2017, leg. FK, KI, KJ, PA; Slava Rusă, 11.V.2017, leg. FK, KI, KJ, PA; Enisala (castle), 20.VI.2019, leg. KI, KJ, PA, TB; Constanţa County: Gura Dobrogei, 30.IV-2.V.2014, leg. KI, IZ, PA, SzL, 12.V.2017, leg. KI, 7.VI.2018, leg. FK, KI, KJ, PA; Cheile Dobrogei, 2.V.2014, leg. KI, IZ, PA, SzL; Hagieni, Pădurea Hagieni (forest), 17.IX.2016, leg. FK, KI, KJ, PA.

\section{Brachysomus (Brachysomus) echinatus (BonSDORFF, 1785)}

New data: Tulcea County: Slava Rusă, 24-25.V.2013, leg. KI, PA, RI, SzL, 20.V.2015, leg. PA, RI, SzK, SzL, 11.V.2017, leg. FK, KI, KJ, PA; Enisala (castle), 21.V.2015, leg. PA, RI, SzK, SzL; M-ţii Măcin, Nifon (forest), 23.VI.2017, leg. KI, KJ, PA, TB.

\section{Brachysomus (Brachysomus) hirtus (BOHEMAN, 1845)} New data: Tulcea County: Slava Rusă, 23.IV.2009, leg. TLA, 4.V.2009, leg. JK, 24-25.V.2013, leg. KI, PA, RI, SzL, 1-3.V.2014, leg. KI, IZ, PA, SzL, 
20.V.2015, leg. PA, RI, SzK, SzL, 11.V.2017, leg. FK, KI, KJ, PA; M-ţii Măcin, Greci env., 6.V.2009, leg. JK; Caugagia, 1.V.2014, leg. KI, IZ, PA, SzL; M-ţii Măcin, Nifon (forest), 23.VI.2017, leg. KI, KJ, PA, TB; 2,5 km from Stejaru to NW, 25.VI.2017, leg. KI, KJ, PA, TB; Constanţa County: Murfatlar, Fântâniţa, 22.V.2015, leg. PA, RI, SzK, SzL.

\section{Brachysomus (Brachysomus) setiger (GYLLENHAL, 1840)}

Published data: Montandon 1906

New data: Tulcea County: Celic Dere, 22-24.V.1997, leg. RS, CH (Coll. Mus. G. AntIPA) - Stejaru env., 5.V.2009, leg. JK; M-ţii Măcin, Greci env., 6.V.2009, leg. JK; Hamcearca, 6.V.2009, leg. JK; 2,7 km from Horia to S, 28.VI.2017, leg. KI; Enisala (castle), 10.V.2017, leg. FK, KI, KJ, PA.

\section{Brachysomus (Hippomias) machaerophorus}

BIALOOKI, 2007

Published data: BIALOOKI 2007

New data: Tulcea County: Codru Monastery, 19.V.2015, leg. PA, 24.VI.2017, leg. KI.

\section{Brachysomus (Hippomias) skodai (BIALOOKI, 2007)} Published data: BIalooki 2007

New data: Constanţa County: $3 \mathrm{~km}$ W of Crucea, Allah Bair (Nature Reserve), 3-4.VI.2010, leg. PJ, SR.

\section{Brachysomus (Hippomias) transsylvanicus}

(SeIDLITZ, 1868)

Published data: Montandon 1908, Bialooki 2007

New data: Tulcea County: „K1st. Kokos Dobrutscha, Breit" coll. A. Podlussány; Hamcearca, 6.V.2009, leg. JK; NE of Topolog, 4.V.2009, leg. JK; M-ţii Măcin, Greci env., 6.V.2009, leg. JK; Slava Rusă, 24-25.V.2013, leg. KI, PA, RI, SzL, 20.V.2015, leg. PA, RI, SzK, SzL; M-ții Măcin, Nifon (forest), 23.VI.2017, leg. KI, KJ, PA, TB; Codru Monastery,
25.VI.2017, leg. KI, KJ, PA, TB; 3 km from Slava Rusă to NE, 24.VI.2017, leg. KI; Constanţa County: Băneasa, Canaraua Fetii, 23.V.2015, leg. PA, RI, SzK, SzL; Cochirleni, 24.V.2015, leg. PA, RI, SzK, SzL.

Eusomatus taeniatus (KRYNICKI, 1834) (= Eusomus laticeps STIERLIN, 1885)

Published data: JaQuet 1899a, Fleck 1905, Negru and RoşCa 1967, RADUTA 2014

Eusomostrophus elongatus (BOHEMAN, 1833)

(= Eusomus elongatus BoHEMAN, 1833)

Published data: JAQUeT 1902b, Fleck 1905

Eusomus beckeri TOURNIER, 1874 (fig. 22)

Published data: JAQUET 1902b, FLECK 1905, MonTANDON 1908

New data: Tulcea County: Insula Popina, Lacul Razelm, 11.V.1992, leg. IM (Coll. Mus. G. ANTIPA) - Babadag (forest), 2.VI.2005, leg. CsS; Constanţa County: Agigea, 23.VI.1960, leg. MW, 27-28.V.1963, leg. XS; Hagieni, 6.VI.1965, leg. MW; Rezervaţia Agigea, 17.VII.2003, leg. MF, 18.VII.2003, leg. RS (Coll. Mus. G. ANTIPA) - Murfatlar, Fântâniţa, 4-5. VII.2014, leg. KI, OA, PA, SzL, 22.V.2015, leg. PA, RI, SzK, SzL, 7-8.VI.2018, leg. FK, KI, KJ, PA; Gura Dobrogei, 7.VI.2018, leg. FK, KI, KJ, PA.

\section{Eusomus ovulum GERMAR, 1823 (fig. 22)}

Published data: MONTANDON 1887, 1906, 1908; JAQUET 1900b; FLECK 1905; Negru 1957, NeGRU and Roşca 1967; TeOdor 1993; TeOdor and ManOle 1996; RADUTA 2014

New data: Tulcea County: M-ții Măcin, Dealurile Pricopanului, 14.VI.2007, leg. CŞ, MC (Coll. Mus. R. ANGheluță); Măcin, leg. ALM, 10.V.2011, leg. IŞI; Măcin, Valea Suluc, 27.V.2005, leg. CB; Greci, 22. 06. 1963, leg. IS; Pădurea Babadag, 6 Martie, 10.V.1992, leg. IM; Celic Dere, 20.V.1997, leg. RS, 20.V.1997, leg. CH, 24.V.1997, 29.V.1997, leg. CP,

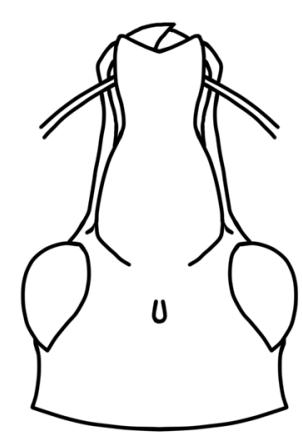

A

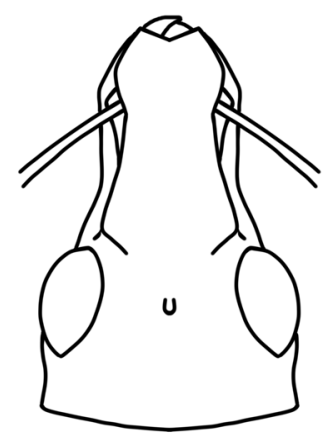

B

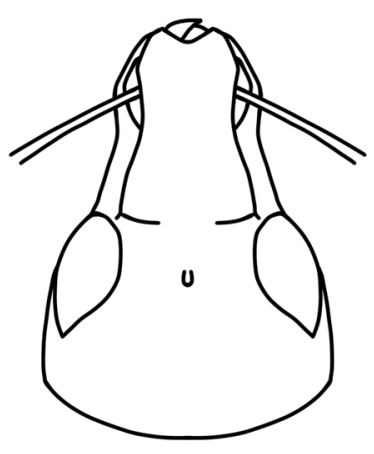

C

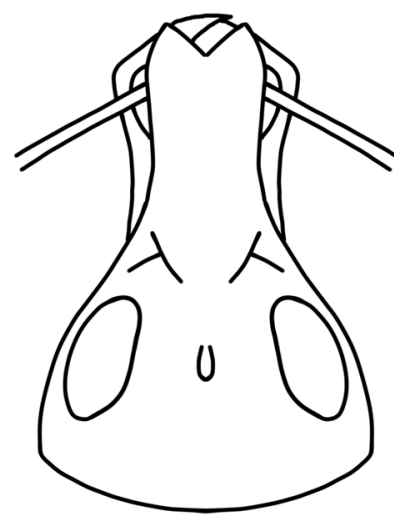

D

Fig. 22. Eusomus ovulum Germar, 1824 and Eusomus beckeri TourniER, 1874 - A: „Eusomus ovulum, Dobrudscha”, Coll. ReITTER; B: „Dobrudscha, ovulum var.”, Coll. ReITTER; C: „Dobrudscha, Eusomus phanophthalmus n. sp.” (unpublished), Coll. ReITter; D: Eusomus beckeri Tournier, 1874, det. A. PodlussánY, 2018 - RO: Dobrogea, prov. Constanţa, MurfatlarFântâniţa, 7-8.VI.2018, leg. K. FetYKó, I. Kocs, J. Kocs and A. PodLussány (Illustration: Viktória SzŐKE). 
24.V.1997, leg. RS, 27.V.2005, leg. RS; Valea Teilor, 22.V.1997, leg. RS, 22.V.1997, leg. CP, 22.V.1997, leg. CH; Ciucurova, 22.V.1997, leg. CH; M-ţii Măcin, Culmea Pricopanului, 31.VII.2006, leg. EP; Babadag, 6.VI.2011, leg. IŞI; Zaghen, 16.V.2013, leg. IŞI (Coll. Mus. G. ANTIPA) - Agighiol, 12.VI.1993, leg. TLA; Beştepe, 13-14.VI.1993, leg. TLA; Canalul Magearu, 17.VI.1993, leg. TLA; Iancina, 11.VI.1993, leg. TLA; Murighiol, Braţul Sfântu Gheorghe, 21-22.VI.2003, leg. TB; M-ţii Măcin (hillside, 200 m), 29.V.2005, leg. CsS; M-ţii Măcin, Valea Fagilor, 30.V.2005, leg. CsS; Frecăţei, Celic Dere Monastery (hillside) 31.V.2005, leg. CsS; M-ţii Măcin, Pricopan, 1.VI.2005, leg. CsS; Babadag (forest), 30.IV.2014, leg. KI, IZ, PA, SzL; Codru Monastery, 23-25.V.2013, leg. KI, PA, RI, SzL, 30.IV-3.V.2014, leg. KI, IZ, PA, SzL, 19.V.2015, leg. PA, RI, SzK, SzL, 10-11.V.2017, leg. FK, KI, KJ, PA, 24-25.VI.2017, leg. KI, KJ, PA, TB, 4.VI.2018, leg. FK, KI, KJ, PA, 20.VI.2019, leg. KI, KJ, PA, TB; Enisala (wooded pasture), 24-25.V.2013, leg. KI, PA, RI, SzL, 29.IV-3.V.2014, leg. KI, IZ, PA, SzL; Enisala (castle), 21.V.2015, leg. PA, RI, SzK, SzL, 10.V.2017, leg. FK, KI, KJ, PA, 20.VI.2019, leg. KI, KJ, PA, TB; Enisala, Cetatea Heraclea, 25.VI.2017, leg. KI, KJ, PA, TB; Slava Rusă, 24-25.V.2013, leg. KI, PA, RI, SzL, 1-3.V.2014, leg. KI, IZ, PA, SzL, 20.V.2015, leg. PA, RI, SzK, SzL, 9.V.2017, leg. FK, KI, KJ, PA; 2 km from Slava Rusă to NE, 3.VI.2018, leg. FK, KI, KJ PA; Greci (village), 8.V.2017, leg. FK, KI, KJ, PA; M-ţii Măcin, Greci, 7.V.2017, leg. FK, KI, KJ, PA, 8.V.2017, leg. FK, KI, KJ, PA, 18-19.VI.2019, leg. KI, KJ, PA, TB; M-ţii Măcin, 5 km S Greci, 8.V.2017, leg. FK, KI, KJ, PA; M-ţii Măcin, Hamcearca, Culmea Cardonului, 19.V.2017, leg. CS; Smârdan, Braţul Măcin, 28.VI.2017, leg. KI, KJ, PA, TB, 18.VI.2019, leg. KI, KJ, PA, TB; N from Nifon, 23.VI.2017, leg. KI, KJ, PA, TB; Turcoaia, $6 \mathrm{~km}$ from Cerna to NW, 24.VI.2017, leg. KI, KJ, PA, TB, 3.VI.2018, leg. FK, KI, KJ PA; Constanţa County: Agigea, 20.VI.1960, 23.VI.1960, leg. MW, 29.VI.1962, leg. ID, 18.VI.1962, leg. AKI, 27-28.V.1963, leg. XS, 15.VI.1964, leg. XS, 20.VI.1964., 18.VII.1964, leg. MW, 22.VI.1964, leg. IC, 12-15.VI.1965, leg. APG, 16-17.VII.2003, leg. MF, 17.VII.2003, leg. RS; Furnica, Rezervația Dumbrăveni, 13-14.V.1995, 22.VI.1995, leg. CP; Negureni, 21.VI.1996, leg. CP; Mangalia Nord, Staţiunea Olimp, 19.VII.2003, leg. GC, MF; Cheile Dobrogei, 12.VI.2012, leg. LM, 29.IV.2013, leg. IŞI (Coll. Mus. G. ANTIPA) - Gura Dobrogei, 28-29. IV.2012, leg. KI, 30.IV-2.V.2014, leg. KI, IZ, PA, SzL, 12.V.2017, leg. FK, KI, KJ, PA, 25.VI.2017, leg. KI, KJ, PA, TB, 7.VI.2018, leg. FK, KI, KJ PA; Murfatlar, Fântâniţa, 4-5.VII.2014, leg. KI, OA, PA, SzL, 22.V.2015, leg. PA, RI, SzK, SzL, 7-8.VI.2018, leg. FK, KI, KJ PA; Balta Limanu, 5-6.VII.2014, leg. KI, OA, PA, SzL; Palazu Mic, 22.V.2015, leg. PA, RI, SzK, SzL; 23 August, 17.IX.2016, leg. FK, KI, KJ, PA; Limanu, 12.V.2017, leg. FK, KI, KJ, PA; Negureni, Pădurea Valea Cişmelelor, 23.V.2015, leg. PA, RI,
SzK, SzL; Băneasa, Canaraua Fetii, 23.V.2015, leg. PA, RI, SzK, SzL; Esechioi (forest), 7.VII.2014, leg. KI, OA, PA, SzL; Cochirleni, 8.VII.2014, leg. KI, OA, PA, SzL, 24.V.2015, leg. PA, RI, SzK, SzL; 2 $\mathrm{km}$ from Sinoie to SE, 27.VI.2017, leg. KI, KJ, PA, TB; Sinoie, Grindul Lupilor, 27.VI.2017, leg. KI, KJ, PA, TB, 5.VI.2018, leg. FK, KI, KJ PA; Istria, Cetatea Histria, 11.V.2017, leg. FK, KI, KJ, PA, 28.VI.2017, leg. KI, KJ, PA, TB.

\section{Exomias holosericeus (FABRICIUS, 1801)}

(= Barypeithes chevrolati BoHEMAN, 1842)

New data: Tulcea County: C. A. Rosetti, VII.1950, leg. IC (Coll. Mus. G. AnTIPA).

\section{Exomias mollicomus (AHRENS, 1812)}

New data: Tulcea County: $2 \mathrm{~km}$ from Sinoie to SE, 27.VI.2017, leg. KI, KJ, PA, TB.

\section{Foucartia dieckmanni ANGELOV, 1986}

\section{Published data: Borovec and Pelletier 2009}

New data: Constanţa County: Mangalia nord VI.1969, leg. ZJN; Mangalia env., Camping Saturn, 8.VI.2005, leg. SR; Mangalia env., Camping Popas Zodiac, 4-6. VI.2008, leg. PJ; Limanu, 12.V.2017, leg. FK, PA.

\section{Foucartia squamulata (HERBST, 1795)}

\section{(= Parafoucartia squamulata HeRBST, 1795)}

Published data: Montandon 1887, JAQUET 1902b, FLECK 1905

New data: Tulcea County: Stejaru env., 5.V.2009, leg. JK; Codru env., 5.V.2009, leg. JK; Codru Monastery, 23-25.V.2013, leg. KI, PA, RI, SzL, 30.IV-3.V.2014, leg. KI, IZ, PA, SzL, 19.V.2015, leg. PA, RI, SzK, SzL, 10-11.V.2017, leg. FK, KI, KJ, PA, 24-25.VI.2017, leg. KI, KJ, PA, TB; Babadag (forest), 30.IV.2014, leg. KI, IZ, PA, SzL; Slava Rusă, 1-3.V.2014, leg. KI, IZ, PA, SzL; $3 \mathrm{~km}$ from Slava Rusă to NE, 24.VI.2017, leg. KI, KJ, PA, TB; 1,5 km from Beidaud to SW, 25.VI.2017, leg. KI; $2 \mathrm{~km}$ from Slava Rusă to NE, 3.VI.2018, leg. FK, KI, KJ, PA; Horea, 3.VI.2018, leg. KI; General Praporgescu, 3.VI.2018, leg. KI; Constanţa County: Gura Dobrogei, 28-29. IV.2012, leg. KI, 26.V.2013, leg. KI, PA, RI, SzL, 30.IV-2.V.2014, leg. KI, IZ, PA, SzL, 12.V.2017, leg. FK, KI, KJ, PA, 27.VI.2017, leg. KI, KJ, PA, TB, 7.VI.2018, leg. FK, KI, KJ, PA; Murfatlar, Fântâniţa, 22.V.2015, leg. PA, RI, SzK, SzL, 7-8.VI.2018, leg. FK, KI, KJ, PA; Limanu, 12.V.2017, leg. FK, KI, KJ, PA.

\section{Paophilus hampei (SEIDLITZ, 1867) \\ Published data: JAQUET 1900b, FLECK 1905}

Sciaphilus asperatus (BONSDORFF, 1785) (= Curculio muricatus FABRICIUS, 1792)

New data: Tulcea County: „Dobrudscha, Sciaphilus muricatus F.", Coll. Reitter (Coll. Hung. Nat. Hist. Mus.) - Babadag (forest), 13.IX.2016, leg. KI, KJ, 
PA, TB; Slava Rusă, 9.V.2017, leg. FK, KI, KJ, PA, 11.V.2017, leg. FK, KI, KJ, PA; Slava Rusă, Uspenia Monastery, 11.V.2017, leg. FK, KI, KJ, PA; Codru Monastery, 10-11.V.2017, leg. FK, KI, KJ, PA; Enisala, 10.V.2017, leg. FK, KI, KJ, PA; M-ţii Măcin, Greci, 7.V.2017, leg. FK, KI, KJ, PA; Greci (village), 8.V.2017, leg. FK, KI, KJ, PA; Smârdan, Braţul Măcin, 22.VI.2017, leg. KI, KJ, PA, TB.

\section{Sciaphobus (Neosciaphobus) squalidus (GYLLENHAL, 1834)}

Published data: JAQUET 1900b, MONTANDON 1908, TeOdor 1993, RAduta 2014

New data: Tulcea County: Babadag, 07.VI.1903, leg. EF; Celic Dere, 24.V.1997, leg. CP; Luncaviţa, Parcul Naţional M-ţii Măcin, 24.V.2005, leg. MS (Coll. Mus. G. ANTIPA); „Dobrudscha”(Coll. Hung. Nat. Hist. Mus.) - Agighiol, 12.VI.1993, leg. TLA; M-ţii Măcin, Valea Fagilor, 30.V.2005, leg. CsS; Slava Rusă, 23.IV.2009, leg. TLA, 24-25.V.2013, leg. KI, PA, RI, SzL, 1-3.V.2014, leg. KI, IZ, PA, SzL, 20.V.2015, leg. PA, RI, SzK, SzL, 31.III.2016, leg. FK, KI, PA, SzL, 9.V.2017, leg. KI; Măcin, Rezervaţia de la Greci, 23.IV.2009, leg. TLA; Codru env., 3.V.2009, leg. JK; M-ţii Măcin, Hamcearca, 6.V.2009, leg. JK; Babadag (forest), 23-25.V.2013, leg. KI, PA, RI, SzL, 30.IV.2014, leg. KI, IZ, PA, SzL, 10.V.2017, leg. KI, 20.V.2017, leg. CS; Codru Monastery, 30.IV3.V.2014, leg. KI, IZ, PA, SzL，30-31.III.2016, leg. FK, KI, PA, SzL; Nifon, pârâul Pârlita valley, 23.VI.2017, leg. KI, KJ, PA, TB; M-ţii Măcin, Nifon (forest), 23.VI.2017, leg. KI, KJ, PA, TB; Greci, 7-8.V.2017, leg. KI; 2 km from Slava Rusă to NE, 3.VI.2018, leg. FK, KI, KJ, PA; Constanţa County: Negureni, 17.V.1994, leg. AP; Furnica, Rezervaţia Dumbrăveni, 13-14.V.1995, leg. CP (Coll. Mus. G. ANTIPA) - Gura Dobrogei, 28-29.IV.2012, leg. KI; Murfatlar, Fântâniţa, 4-5.VII.2014, leg. KI, OA, PA, SzL; Băneasa, Canaraua Fetii (quarry), 23.V.2015, leg. PA, RI, SzK, SzL, 2-3.IV.2016, leg. FK, KI, PA, SzL; Esechioi (forest), 1-2.IV.2016, leg. FK, KI, PA, SzL; Bugeac, 2.IV.2016, leg. FK, KI, PA, SzL; Ostrov, 2.IV.2016, leg. FK, KI, PA, SzL; Cochirleni, 3.IV.2016, leg. FK, KI, PA, SzL.

Sitonini Gistel, 1848

Charagmus griseus (FABRICIUS, 1775)

Published data: JAQUET 1900b, FLECK 1905

\section{Sitona callosus GyLLENHAL, 1834}

Published data: Montandon 190,; Negru and RoşCa 1967, RAduTa 2014

New data: Tulcea County: M-ţii Măcin-Valea lui Moş Enache, 5.X.2007, leg. CI, CŞ (Coll. Mus. R. Angheluță); Letea, 15.VII.1962, leg. MW; Periprava, Pădurea Letea, 23.X.1963, leg. APG; Pădurea Letea, 9.V.1964, leg. IS, 22.IX.2010, leg. IŞI; Sulina, 24.VII.1964, leg. ŞN; Periprava, 1.VII.1965., 4.VII.1965, leg. ŞN, 14.VII.1966, leg. ŞN, 14.VII.1966, leg. XS; Sulina, 12.VII.1967, leg. AtR; Caraorman, Deltă, 15.VI.1969, leg. ŞN; Somova, 28.VII.1987, leg. IM; Celic Dere, 20.V.1997, leg. RS (Coll. Mus. G. Antipa) - Beştepe, 30.IV.1993, leg. TLA; Codru env., 23.IX.2010, leg. JK; Codru Monastery, 2325.V.2013, leg. KI, PA, RI, SzL, 13.IX.2016, leg. KI, KJ, PA, TB; Enisala (castle), 29.IV-3.V.2014, leg. KI, IZ, PA, SzL, 22.IX.2015, leg. KI, KJ, PA; Parcheş, 23.IX.2015, leg. KI, KJ, PA; Slava Rusă 23.IX.2015, leg. KI, KJ, PA, 31.III.2016, leg. FK, KI, PA, SzL; Enisala (wooded pasture), 13.IX.2016, leg. TB; Smârdan, Braţul Măcin, 7.V.2017, leg. FK, KI, KJ, PA; 1,5 km from Beidaud to SW, 25.VI.2017, leg. KI, KJ, PA, TB; Enisala, Cetatea Heraclea, 25.VI.2017, leg. KI, KJ, PA, TB; Nifon, pârâul Pârlita valley, 23.VI.2017, leg. KI, KJ, PA, TB; Turcoaia, $6 \mathrm{~km}$ from Cerna to NW, 24.VI.2017, leg. KI; 2,7 km from Horia to S, 28.VI.2017, leg. KI, KJ, PA, TB; M-ţii Măcin, Greci, 18-19.VI.2019, leg. KI, KJ, PA, TB; Constanţa County: Valul lui Traian, 18.X.1953, leg. XS, 7.V.1955, leg. GB, 19.VII.1955, leg. VR; Negureni, 25.VI.1995, leg. CP; Pădurea Hagieni, 15.VII.1997, leg. IoS (Coll. Mus. G. ANTIPA) $-3 \mathrm{~km} \mathrm{~W}$ of Crucea, Allah Bair (Nature Reserve), 1.V.2009, leg. JK, 26.IX.2015, leg. KI, KJ, PA; Murfatlar, Fântâniţa , 4-5.VII.2014, leg. KI, OA, PA, SzL, 24-25.IX.2015, leg. KI, KJ, PA, 14.IX.2016, leg. KI, KJ, PA, TB; Esechioi (forest), 7.VII.2014, leg. KI, OA, PA, SzL; Gura Dobrogei, 25-27.VI.2017, leg. KI, KJ, PA, TB, 7.VI.2018, leg. FK, KI, KJ, PA; Istria, Cetatea Histria, 26-28. VI.2017, leg. KI, KJ, PA, TB, 21.VI.2019, leg. KI, KJ, PA, TB; Sinoie, Grindul Lupilor, 27.VI.2017, leg. KI, KJ, PA, TB, 22.VI.2019, leg. KI, KJ, PA, TB; $2 \mathrm{~km}$ from Sinoie to SE, 27.VI.2017, leg. KI, 21, 23.VI.2019, leg. KI; Crucea, 9.VI.2018, leg. FK, KI, KJ, PA.

Sitona cylindricollis cylindricollis FÅHRAEUS, 1840 Published data: Montandon 1908, Negru and RoşCa 1967, TeOdor and Manole 1996, Raduta 2014 New data: Tulcea County: Babadag, 29.VI.1956, leg. XS; Măcin, 23.V.1961; Pădurea Letea, 4.V.2012, leg. IŞI (Coll. Mus. G. ANTIPA) - Măcin (hillside, 200 m), 29.V.2005, leg. CsS; M-ţii Măcin, Valea Fagilor, 30.V.2005, leg. CsS; Babadag (forest), 2.VI.2005, leg. CsS; Enisala, 24-25.V.2013, leg. KI, PA, RI, SzL; Codru Monastery, 30-31.III.2016, leg. FK, KI, PA, SzL; Smârdan, Braţul Măcin, 22.VI.2017, leg. KI, KJ, PA, TB; Enisala (castle), 20.VI.2019, leg. KI, KJ, PA, TB; Constanţa County: Furnica, Rezervaţia Dumbrăveni, 13-14.V.1995, leg. CP (Coll. Mus. G. ANTIPA) - Murfatlar, Fântâniţa, 4-5.VII.2014, leg. KI, OA, PA, SzL; Băneasa, Canaraua Fetii, 23.V.2015, leg. PA, RI, SzK, SzL; Istria, Cetatea Histria, 11.V.2017, leg. FK, KI, KJ, PA, 26-28.VI.2017, leg. KI, KJ, PA, TB; 23 August, 17.IX.2016, leg. FK, KI, KJ, PA; Costineşti (coast), 17.IX.2016, leg. KI; 2 km from Sinoie to SE, 5.VI.2018, leg. FK, KI, KJ, PA. 
Sitona discoideus GYLLENHAL, 1834

Published data: MonTANDON 1887

Sitona hispidulus (FABRICIUS, 1777)

New data: Tulcea County: Celic Dere, 20.V.1997, leg. CH (Coll. Mus. G. ANTIPA) - Canalul Magearu, 17.VI.1993, leg. TLA; Frecăţei, Celic Dere Monastery (hillside) 31.V.2005, leg. CsS; Enisala, 29.IV3.V.2014, leg. KI, IZ, PA, SzL; Parcheş 23.IX.2015, leg. KI, KJ, PA; Slava Rusă, 11.V.2017, leg. KI; Smârdan, Braţul Măcin, 18.VI.2019, leg. KI, KJ, PA, TB; Constanţa County: Ostrov, 2.IV.2016, leg. FK, KI, PA, SzL.

\section{Sitona humeralis STEPHENS, 1831}

Published data: RADUTA 2014

New data: Tulcea County: M-ţii Măcin, Valea lui Moş Kalâm, 5.X.2007, leg. CŞ, CI (Coll. Mus. R. Angheluță); Sulina, 12.VII.1967, leg. AtR; C. A. Rosetti, Delta Dunării, 9.VI.1981, leg. CP; Celic Dere, 20.V.1997, leg. CH (Coll. Mus. G. Antipa) - Periprava, 1.VII.2005, leg. DDC; Parcheş, 23.IX.2015, leg. KI, KJ, PA; Sălcioara, Lacul Sălcioara, 13.IX.2016, leg. KI, KJ, PA, TB; Smârdan, Braţul Măcin, 11.IX.2016, leg. KI, KJ, PA, TB, 7.V.2017, leg. FK, KI, KJ, PA; N from Nifon, 23.VI.2017, leg. KI, KJ, PA, TB; Enisala, Cetatea Heraclea, 25.VI.2017, leg. KI, KJ, PA, TB; Nifon, pârâul Pârlita valley, 23.VI.2017, leg. KI, KJ, PA, TB; M-ții Măcin, Greci, 7.V.2017, leg. KI; M-ții Măcin, 5 km S Greci, 8.V.2017, leg. FK, KI, KJ, PA; S from Hamcearca, 24.VI.2017, leg. KI; Turcoaia, 6 $\mathrm{km}$ from Cerna to NW, 24.VI.2017, leg. KI; 1,5 km from Beidaud to SW, 25.VI.2017, leg. KI; Ciucurova, 3.VI.2018, leg. FK, KI, KJ, PA; 2 km from Slava Rusă to NE, 3.VI.2018, leg. FK, KI, KJ. PA; Codru Monastery, 4.VI.2018, leg. FK, KI, KJ, PA; Enisala (castle), 20.VI.2019, leg. KI; Constanţa County: Agigea, 18.VI.1962, leg. AtR; Hagieni, 29.X.1965, leg. ŞN; Eforie Nord, 19.VI.1966, leg. MaC; Valul lui Traian, 26.V. ????, leg. XS (Coll. Mus. G. ANTIPA) - Istria env., Cetatea Histria, 19.IX.2010, leg. JK; Murfatlar, Fântânița, 4-5.VII.2014, leg. KI，OA, PA, SzL, 24-25.IX.2015, leg. KI, KJ, PA; Esechioi (forest), 7.VII.2014, leg. KI, OA, PA, SzL; Băneasa, Canaraua Fetii (quarry), 7.VII.2014, leg. KI, OA, PA, SzL; Bugeac 16.IX.2016, leg. KI, KJ, PA, TB; Albeşti, Pădurea Hagieni (forest), 12.V.2017, leg. FK, KI, KJ, PA; Hagieni, Pădurea Hagieni (forest), 17.IX.2016, leg. FK, KI, KJ, PA; 2 km from Sinoie to SE, 27.VI.2017, leg. KI, KJ, PA, TB, 5.VI.2018, leg. FK, KI, KJ, PA, 21, 23.VI.2019, leg. KI; Sinoie, Grindul Lupilor, 27.VI.2017, leg. KI, KJ, PA, TB, 22.VI.2019, leg. KI, KJ, PA, TB; Costineşti (coast), 17.IX.2016, leg. FK, KI, KJ, PA; Pecineaga (forest), 14.V.2017, leg. KI; Istria, Cetatea Histria, 6.VI.2018, leg. FK, KI, KJ, PA, 21.VI.2019, leg. KI, KJ, PA, TB; Gura Dobrogei, 7.VI.2018, leg. FK, KI, KJ, PA.

Sitona inops SCHÖNHERR, 1832
New data: Tulcea County: M-ţii Măcin, Valea lui Moș Kalâm, 5.X.2007, leg. CI (Coll. Mus. R. ANGHELuȚă); Cerna, 26.V.2005, leg. CB (Coll. Mus. G. ANTIPA) - M-ții Măcin, to the Peak Țuţuiatu, 28.IV.2010, leg. TLA; Codru Monastery, 23-25.V.2013, leg. KI, PA, RI, SzL, 19.V.2015, leg. PA, RI, SzK, SzL, 10-11.V.2017, leg. FK, KI, KJ, PA, 24-25.VI.2017, leg. KI, KJ, PA, TB; Enisala, 24-25.V.2013, leg. KI, PA, RI, SzL, 29.IV-3.V.2014, leg. KI, IZ, PA, SzL; Slava Rusă, 1-3.V.2014, leg. KI, IZ, PA, SzL; Smârdan, Braţul Măcin, 21-23.IX.2015, leg. KI, KJ, PA, 7.V.2017, leg. FK, KI, KJ, PA; Enisala (castle), 10.V.2017, leg. FK, KI, KJ, PA, 20.VI.2019, leg. KI, KJ, PA, TB; M-ţii Măcin, 5 km S Greci, 8.V.2017, leg. FK, KI, KJ, PA; Constanţa County: Esechioi (forest), 7.VII.2014, leg. KI, OA, PA, SzL; Gura Dobrogei, 30.IV-2.V.2014, leg. KI, IZ, PA, SzL; Băneasa, Canaraua Fetii, 23.V.2015, leg. PA, RI, SzK, SzL; Cochirleni, 3.IV.2016, leg. FK, KI, PA. SzL; Pecineaga (forest), 14.V.2017, leg. FK, KI, KJ, PA; 2 $\mathrm{km}$ from Sinoie to SE, 27.VI.2017, leg. KI.

\section{Sitona languidus GYLLENHAL, 1834}

New data: Tulcea County: Babadag, Valea Caugagiei, 17.V.1963, leg. IC (Coll. Mus. G. Antipa) - Slava Rusă, 1-3.V.2014, leg. KI, IZ, PA, SzL.

\section{Sitona lateralis GYLLENHAL, 1834 (= Sitona ononidis} SHARP, 1867)

New data: Tulcea County: Smârdan, Braţul Măcin, 7.V.2017, leg. FK, KI, KJ, PA; Babadag (forest), 20.V.2017, leg. CS; Constanţa County: Ostrov, Lacul Bugeac, 16.IX.2016, leg. TB.

\section{Sitona lineatus (LINNÉ, 1758)}

Published data: MonTANDON 1887, JAQUET 1900b, Fleck 1905, Teodor and MANole 1996

New data: Tulcea County: Periprava, 1.VII.2005, leg. DDC; Codru Monastery, 30-31.III.2016, leg. FK, KI, PA, SzL, 24-25.VI.2017, leg. KI; M-ţii Măcin, 5 km S Greci, 8.V.2017, leg. FK, KI, KJ, PA; Smârdan, Braţul Măcin, 22.VI.2017, leg. KI, KJ, PA, TB; Turcoaia, 6 $\mathrm{km}$ from Cerna to NW, 24.VI.2017, leg. KI; Constanţa County: Hagieni, 17-22.VII.1997, leg. CP; Cotu Văii, 23.VII.1997, leg. CP; Rezervaţia Naturală Agigea, 17.VII.2003, leg. MF (Coll. Mus. G. ANTIPA) Murfatlar, Fântâniţa, 4-5.VII.2014, leg. KI, OA, PA, SzL, 24-25.IX.2015, leg. KI, KJ, PA, 7-8.VI.2018, leg. FK, KI, KJ, PA; Balta Limanu, 5-6.VII.2014, leg. KI, OA, PA, SzL; Pădurea Hagieni (forest), 6.VII.2014, leg. KI, OA, PA, SzL, 12.V.2017, leg. FK, KI, KJ, PA; Limanu, 12.V.2017, leg. FK, KI, KJ, PA; Hagieni, Pădurea Hagieni (forest), 17.IX.2016, leg. FK, KI, KJ, PA; 23 August, 17.IX.2016, leg. FK, KI, KJ, PA; Pecineaga (forest), 14.V.2017, leg. FK, KI, KJ, PA; Gura Dobrogei, 25-27.VI.2017, leg. KI, KJ, PA, TB; Istria, Cetatea Histria, 26-28.VI.2017, leg. KI, KJ, PA, TB; $2 \mathrm{~km}$ from Sinoie to SE, 27.VI.2017, leg. KI, KJ, PA, TB; Sinoie, Grindul Lupilor, 27.VI.2017, leg. 
KI, KJ, PA, TB; Vadu, Grindul Chituc, 22.VI.2019, leg. KI, KJ, PA, TB.

\section{Sitona longulus GYLLENHAL, 1834}

New data: Tulcea County: M-ţii Măcin, Pricopan (370 m), 13.VI.2004, leg. MM (Coll. Mus. R. ANGHELUŢĂ) - 1,5 km from Beidaud to SW, 25.VI.2017, leg. KI, KJ, PA, TB; $2,7 \mathrm{~km}$ from Horia to S, 28.VI.2017, leg. KI, KJ, PA, TB; Nifon, pârâul Pârlita valley, 23.VI.2017, leg. KI, KJ, PA, TB; M-ţii Măcin, $5 \mathrm{~km}$ S Greci, 8.V.2017, leg. KI; Turcoaia, 6 km from Cerna to NW, 3.VI.2018, leg. FK, KI, KJ, PA, 19.VI.2019, leg. KI, KJ, PA, TB; Smârdan, Braţul Măcin, 18.VI.2019, leg. KI, KJ, PA, TB; Enisala (castle), 20.VI.2019, leg. KI, KJ, PA, TB; Constanţa County: Valul lui Traian, 16.VII.1955., 29.VI.1961, leg. MW; Comorova, 31.VIII.1963, leg. AtR; Agigea, 4.VII.1977, leg. MA, GA (Coll. Mus. G. ANTIPA) Murfatlar, Fântâniţa, 4-5.VII.2014, leg. KI, OA, PA, SzL; Balta Limanu, 5-6.VII.2014, leg. KI, OA, PA, SzL; Esechioi (forest), 7.VII.2014, leg. KI, OA, PA, SzL; Oltina (village), 8.VII.2014, leg. KI, OA, PA, SzL; Băneasa, Canaraua Fetii (quarry), 7.VII.2014, leg. KI, OA, PA, SzL; Ion Corvin, 15.IX.2016, leg. KI, KJ, PA, TB; M-ţii Măcin, 5 km S Greci, 8.V.2017, leg. KI;

\section{Sitona macularius macularius (MARSHAM, 1802)}

Published data: JAQUET 1899b, FLECK 1905, Montandon 1908, Negru 1957, Negru and RoşCa 1967, IENIŞTEA 1974, TeOdOR 1993, TeOdor and MANOLE 1996, Raduta 2014

New data: Tulcea County: Babadag, Visterna, 15-16.V.1963, leg. IC; Visterna, Valea Caugagiei, 17.V.1963, leg. IC; Periprava, Delta, 24.VI.1966, leg. ŞN, 14.VII.1966, leg. XS; Sulina, 10.IX.1966, leg. XS, 12.VII.1967, leg. AtR; Crişan, Delta Dunării, 13.VII.1970, leg. APG; Tulcea, 27.X.1970, leg. ŞN; Caraorman, 15.V.1992, leg. NG; Pădurea Letea, Delta Dunării, 18.V.1993, leg. IM; Celic Dere, 20.V.1997, leg. CH; Ciucurova, 22.V.1997, leg. CH (Coll. Mus. G. AntIPA) - Agighiol, 1.V.1993, leg. TLA; Beştepe, 30.IV.1993, leg. TLA; Pădurea Letea (forest), 17.VI.1993, leg. TLA; Periprava, 1.VII.2005, leg. DDC; M-ţii Măcin, Luncaviţa, 30.V.2005, leg. CsS; Enisala (castle), 29.IV-3.V.2014, leg. KI, IZ, PA, SzL, 30-31.III.2016 leg. FK, KI, PA, SzL, 10.V.2017, leg. FK, KI, KJ, PA, 20.VI.2019, leg. KI, KJ, PA, TB; Codru Monastery, 30-31.III.2016, leg. FK, KI, PA, SzL, 10-11.V.2017, leg. FK, KI, KJ, PA, 24-25. VI.2017, leg. KI, KJ, PA, TB, 20.VI.2019, leg. KI, KJ, PA, TB; Slava Rusă, 31.III.2016, leg. FK, KI, PA, SzL, 9.V.2017, leg. FK, KI, KJ, PA, 11.V.2017, leg. FK, KI, KJ, PA; Greci (village), 8.V.2017, leg. FK, KI, KJ, PA; Smârdan, Braţul Măcin, 22.VI.2017, leg. KI, KJ, PA, TB, 18.VI.2019, leg. KI, KJ, PA, TB; Jijila, râul Jijila, 23.VI.2017, leg. KI, KJ, PA, TB; Nifon, pârâul Pârlita valley, 23.VI.2017, leg. KI, KJ, PA, TB; N from Nifon, 23.VI.2017, leg. KI, KJ, PA,
TB; Turcoaia, $6 \mathrm{~km}$ from Cerna to NW, 24.VI.2017, leg. KI, KJ, PA, TB, 3.VI.2018, leg. FK, KI, KJ, PA, 19.VI.2019, leg. KI, KJ, PA, TB; 1,5 km from Beidaud to SW, 25.VI.2017, leg. KI, KJ, PA, TB; Enisala, Cetatea Heraclea, 25.VI.2017, leg. KI, KJ, PA, TB; $2,7 \mathrm{~km}$ from Horia to S, 28.VI.2017, leg. KI, KJ, PA, TB; 2,5 km from Stejaru to NW, 25.VI.2017, leg. TB, KI; Horea, 3.VI.2018, leg. FK, KI, KJ, PA; General Praporgescu, 3.VI.2018, leg. FK, KI, KJ, PA; M-ţii Măcin, Greci, 18-19.VI.2019, leg. KI, KJ, PA, TB; Enisala (forest), 20.VI.2019, leg. KI, KJ, PA, TB; Constanţa County: Agigea, 27.V.1963, leg. XS, 15.VI.1964, leg. XS, 22.VI.1964, leg. MW; Pădurea Hagieni, 28.X.1965, leg. ŞN, 17.VII.1997, leg. IoS, 17-22.VII.1997, leg. CP; Băneasa, Canaraua Fetii, 20.V.1993, leg. AP; Furnica, Rezervaţia Dumbrăveni, 13-14.V.1995, leg. CP; Negureni, 25.VI.1995, leg. CP (Coll. Mus. G. ANTIPA) - Gura Dobrogei, 30.IV2.V.2014, leg. KI, IZ, PA, SzL, 1.IV.2016, leg. FK, KI, PA, SzL, 12.V.2017, leg. FK, KI, KJ, PA, 25-27. VI.2017, leg. KI, KJ, PA, TB, 7.VI.2018, leg. FK, KI, KJ, PA; Murfatlar, Fântâniţa, 4-5.VII.2014, leg. KI, OA, PA, SzL, 22.V.2015, leg. PA, RI, SzK, SzL, 2425.IX.2015, leg. KI, KJ, PA, 7-8.VI.2018, leg. FK, KI, KJ, PA; Balta Limanu, 5-6.VII.2014, leg. KI, OA, PA, SzL; Pădurea Hagieni (forest), 6.VII.2014, leg. KI, OA, PA, SzL; Albeşti, Pădurea Hagieni, 12.V.2017, leg. FK, KI, KJ, PA; Esechioi (forest), 7.VII.2014, leg. KI, OA, PA, SzL, 1-2.IV.2016, leg. FK, KI, PA, SzL; Palazu Mic, 22.V.2015, leg. PA, RI, SzK, SzL; Băneasa, Canaraua Fetii (quarry), 23.V.2015, leg. PA, RI, SzK, SzL, 2-3.IV.2016, leg. FK, KI, PA, SzL; Cochirleni, 24.V.2015, leg. PA, RI, SzK, SzL, 3.IV.2016, leg. FK, KI, PA, SzL; Negru Vodă (Nature Reserve) 25.IX.2015, leg. KI, KJ, PA; Crucea, Allah Bair (Nature Reserve) 26.IX.2015, leg. KI, KJ, PA; Ostrov, 2.IV.2016, leg. FK, KI, PA, SzL; Limanu, 12.V.2017, leg. FK, KI, KJ, PA; Pecineaga (forest), 14.V.2017, leg. FK, KI, KJ, PA; Istria, Cetatea Histria, 26-28.VI.2017, leg. KI, KJ, PA, TB; 2 km from Sinoie to SE, 27.VI.2017, leg. KI, KJ, PA, TB, 21, 23.VI.2019, leg. KI; Sinoie, Grindul Lupilor, 27.VI.2017, leg. KI, KJ, PA, TB, 22.VI.2019, leg. KI, KJ, PA, TB; Crucea, 9.VI.2018, leg. FK, KI, KJ, PA; Vadu, Grindul Chituc, 22.VI.2019, leg. KI, KJ, PA, TB.

Sitona obsoletus obsoletus (GMELIN, 1790) (= lepidus GYLlenHaL, 1834)

Published data: Montandon 1887, JAQuet 1899b, Hurmuzachi 1904, Fleck 1905, Negru and RoşCa 1967, RAdUTA 2014

New data: Tulcea County: $1.5 \mathrm{~km}$ N of Horia, Lacul Horia (lake), 24.IX.2010, leg. JK; Constanţa County: Istria, Cetatea Histria, 26-28.VI.2017, leg. KI, KJ, PA, TB; $2 \mathrm{~km}$ from Sinoie to SE, 27.VI.2017, leg. KI, $\mathrm{KJ}, \mathrm{PA}, \mathrm{TB}$.

*Sitona ophthalmicus DesBrochers des Loges, 1869 New data: Tulcea County: M-ţii Măcin, Valea lui 
Arsu, 5.X.2007, leg. CI (Coll. Mus. R. Angheluţă).

Sitona puncticollis STEPHENS, 1831

Published data: Negru 1957, Negru and RoşCa 1967, RADUTA 2014

New data: Tulcea County: M-ţii Măcin, Dealurile Pricopanului, 14.VI.2007, leg. CŞ, MC (Coll. Mus. R. ANGHELUȚĂ) - Măcin (hillside, 200 m), 29.V.2005, leg. CsS; M-ţii Măcin, Valea Fagilor (dried lake bed) 30.V.2005, leg. CsS; M-ţii Măcin, Valea Fagilor (quarry) 30.V.2005, leg. CsS; Frecăţei, Celic Dere Monastery (hillside), 31.V.2005, leg. CsS; M-ţii Măcin, Horia, 1.VI.2005, leg. CsS; Enisala, 29.IV3.V.2014, leg. KI, IZ, PA, SzL; Babadag (forest), 13.IX.2016, leg. KI, KJ, PA, TB, 20.V.2017, leg. CS; Enisala (wooded pasture), 13.IX.2016, leg. KI, KJ, PA, TB; Smârdan, Braţul Măcin, 7.V.2017, leg. FK, KI, KJ, PA, 28.VI.2017, leg. KI, KJ, PA, TB; Enisala (castle), 10.V.2017, leg. FK, KI, KJ, PA, 20.VI.2019, leg. KI, KJ, PA, TB; Nifon, pârâul Pârlita valley, 23.VI.2017, leg. KI, KJ, PA, TB; N from Nifon, 23.VI.2017, leg. KI, KJ, PA, TB; Codru Monastery, 24-25.VI.2017, leg. KI, KJ, PA, TB; $1,5 \mathrm{~km}$ from Beidaud to SW, 25.VI.2017, leg. KI, KJ, PA, TB; Enisala, Cetatea Heraclea, 25.VI.2017, leg. KI, KJ, PA, TB; 3 km from Slava Rusă to NE, 24.VI.2017, leg. TB; Turcoaia, $6 \mathrm{~km}$ from Cerna to NW, 24.VI.2017, leg. KI; M-ţii Măcin, 5 km S Greci, 8.V.2017, leg. KI; M-ţii Măcin, Greci, 18-19. VI.2019, leg. KI, KJ, PA, TB; Constanţa County: Furnica, Rezervaţia Dumbrăveni, 13-14.V.1995, 22.VI.1995, leg. CP (Coll. Mus. G. ANTIPA) - Gura Dobrogei, 28-29.IV.2012, leg. KI, 25-27.VI.2017, leg. KI, KJ, PA, TB, 7.VI.2018, leg. FK, KI, KJ, PA; Esechioi (forest), 7.VII.2014, leg. KI, OA, PA, SzL, 7.VII.2014, leg. KI, OA, PA, SzL; Esechioi (semidry steppic grassland on loess), 16.IX.2016, leg. KI, KJ, PA, TB; Murfatlar, Fântâniţa, 4-5.VII.2014, leg. KI, OA, PA, SzL, 7-8.VI.2018, leg. FK, KI, KJ, PA; Băneasa, Canaraua Fetii (quarry), 7.VII.2014, leg. KI, OA, PA, SzL; Istria, Cetatea Histria, 14.IX.2016, leg. KI, KJ, PA, TB, 26-28.VI.2017, leg. KI, KJ, PA, TB; $2 \mathrm{~km}$ from Sinoie to SE, 27.VI.2017, leg. KI, KJ, PA, TB, 5.VI.2018, leg. FK, KI, KJ, PA; Sinoie, Grindul Lupilor, 27.VI.2017, leg. KI, KJ, PA, TB, 5.VI.2018, leg. FK, KI, KJ, PA.

Sitona striatellus GyLlENHAL, 1834

New data: Tulcea County: Enisala, 24-25.V.2013, leg. KI, PA, RI, SzL.

Sitona sulcifrons sulcifrons (THUNBERG, 1798)

Published data: Montandon 1887, JAQUET 1904, Fleck 1905, Negru and Roşca 1967, Teodor and Manole 1996, Raduta 2014

New data: Tulcea County: Enisala, 29.IV-3.V.2014, leg. KI, IZ, PA, SzL; Smârdan, Braţul Măcin, 7.V.2017, leg. FK, KI, KJ, PA.
Sitona sulcifrons deubeli KraUSS, 1902

New data: Tulcea County: Canalul Magearu, 17.VI.1993, leg. TLA; M-ţii Măcin, Luncaviţa, 30.V.2005, leg. CsS; Frecăţei, Celic Dere Monastery (hillside), 31.V.2005, leg. CsS; $1.5 \mathrm{~km} \mathrm{~N}$ of Horia, Lacul Horia (lake), 24.IX.2010, leg. JK; Nifon, pârâul Pârlita valley, 23.VI.2017, leg. KI, KJ, PA, TB; Smârdan, Braţul Măcin, 18.VI.2019, leg. KI; Constanţa County: Ostrov, Lacul Bugeac, 16.IX.2016, leg. KI, TB.

\section{Sitona suturalis STEPHENS, 1831}

New data: Tulcea County: Celic Dere, 24.V.1997, leg. CP (Coll. Mus. G. ANTIPA) - Periprava, 1.VII.2005, leg. DDC; M-ţii Măcin, to the Peak Ţuţuiatu, 28.IV.2010, leg. TLA; Enisala (castle), 13.IX.2016, leg. KI, KJ, PA, TB; Nifon, pârâul Pârlita valley, 23.VI.2017, leg. KI, KJ, PA, TB; Constanţa County: Ostrov, Lacul Bugeac, 16.IX.2016, leg. KI, KJ, PA, TB.

\section{Sitona waterhousei WALTON, 1846}

New data: Tulcea County: Măcin (hillside, $200 \mathrm{~m}$ ), 29.V.2005, leg. CsS; Parcheş 23.IX.2015, leg. KI, KJ, PA.

Tanymecini LACORDAIRE, 1863

Tanymecina LACORDAIRE, 1863

Chlorophanus flavescens (FABRICIUS, 1787)

(= graminicola $\mathrm{SCHÖNHERR,} \mathrm{1832)}$

Published data: MontANDON 1908

New data: Tulcea County: Delta Dunării, Crișan, 26.VII.2003, leg. GP (Coll. Mus. R. ANGHeluță).

\section{Chlorophanus pollinosus (FABRICIUS, 1792)}

(= gibbosus PAYKULL, 1792)

Published data: JAQUeT 1900b; FleCK 1905; TeOdOR and ManOle 1996; Raduta 2014

New data: Tulcea County: Gorgova, 16.VI.1993, leg. TLA.

*Chlorophanus viridis balcanicus BEHNE, 1989

New data: Tulcea County: Smârdan, Braţul Măcin, 28.VI.2017, leg. TB.

Cycloderes (Cycloderes) pilosulus (HERBST, 1795)

Published data: Montandon 1887, 1908; JAQUET 1900b; FleCK 1905; Negru and RoşCA 1967

New data: Tulcea County: M-ţii Măcin, Dealul Stănilă, 13.VI.2007, leg. MC (Coll. Mus. R. Angheluță); Insula Popina, Lacul Razelm, 11. 05. 1992, leg. IM (Coll. Mus. G. ANTIPA) - Măcin (hillside, 200 m), 29.V.2005, leg. CsS; Turcoaia, 24.IV.2009, leg TLA, 19.VI.2019, leg. KI, KJ, PA, TB; Atmagea env., 24.IX.2010, leg. JK; Enisala, 24-25.V.2013, leg. KI, PA, RI, SzL; Enisala (castle), 20.VI.2019, leg. KI, KJ, PA, TB; Constanţa County: Hârşova, leg. ALM (Coll. Mus. G. ANTIPA) - Cochirleni, 8.VII.2014, leg. KI, OA, PA, SzL. 
Tanymecus (Episomecus) dilaticollis GYLLENHAL, 1834

Published data: JAQUET 1898b, 1902b; FLECK 1905; Montandon 1908; Negru and Roşca 1967; Negru 1968; Ciochia 1985; Teodor 1993; Teodor and MANole 1996; RAduta 2014

New data: Tulcea County: C. A. Rosetti, 10.V.1964, leg. XS; Sfântu Gheorghe, 10-18.VII.1974; Insula Popina, Lacul Razelm, 11.V.1992, leg. IM; Maliuc, Mila 26, 18-19.V.1992, leg. IM; Enisala, 12.VI.1993, leg. IM; Dunavăţ, Delta Dunării, 31.V.1994, leg. CH; Celic Dere, 20.V.1997, leg. CH, RS, 24.V.1997., 29.V.1997, leg. CP; Izvoarele, 21.V.1997, leg. RS; Niculiţel, Mănăstirea Cocoş, 24.V.2005, leg. RS, IM (Coll. Mus. G. Antipa) - Agighiol, 12.VI.1993, leg. TLA; Beştepe, 13.VI.1993, leg. TLA; Canalul Magearu, 17.VI.1993, leg. TLA; Enisala (forest), 11.VI.1993, leg. TLA, 24-25.V.2013, leg. KI, PA, RI, SzL, 29.IV-3.V.2014, leg. KI, IZ, PA, SzL, 4.VI.2018, leg. FK, KI, KJ, PA; Iancina, 11.VI.1993, leg. det. TLA; Braţul Sfântu Gheorghe, 21-22.VI.2003, leg. TB; Măcin (hillside, 200 m), 29.V.2005, leg. CsS; M-ţii Măcin, Valea Fagilor (quarry), 30.V.2005, leg. CsS; M-ţii Măcin, Pricopan, 1.VI.2005, leg. CsS; Babadag (forest), 2.VI.2005, leg. CsS; Atmagea, 28.IV.2012, leg. KI; Slava Rusă, 20.V.2015, leg. PA, RI, SzK, SzL; Enisala (castle), 21.V.2015, leg. PA, RI, SzK, SzL, 10.V.2017, leg. FK, KI, KJ, PA; Smârdan, Braţul Măcin, 7.V.2017, leg. FK, KI, KJ, PA, 28.VI.2017, leg. KI, KJ, PA, TB, 2.VI.2018, leg. FK, KI, KJ, PA; Constanţa County: - Valul lui Traian, 21.VI.1958, leg. AtR; Agigea, 29.V.1962, leg. ID, 27.V.1963, 29.V.1963, leg. XS, 12.VI.1964, leg. NS, 24.VI.1964, leg. MW, VI.1964, leg.IC; Mangalia, Pădurea Comorova, 30.V.1963, leg. XS; Hagieni, 04.VI.1964, leg. NS, 6.VI.1965, leg. ŞN, 06.VII.1965, leg. MW, 16.VII.1997, leg. CP; Băneasa, Cogeacu, 29.V.1965, leg. ŞN, MW; Băneasa, Canaraua Fetii, 29.V.1965, leg. XP, 17.V.1972, leg. NS, 18.V.1993, leg. CP, 19.V.1993, leg. MB, 20.V.1993, leg. CP, CH, AP, 26.V.1995, leg. CP, 10.IV.1999, leg. ŞP; Băneasa, 30.V.1965, leg. MW; Băneasa, Iortmac, 01-03. VI.1965, leg. ŞN; Furnica, Pădurea Dumbrăveni, 13-14.V.1995, leg. CP, GA, 22.VI.1995, leg. CP (Coll. Mus. G. AnTiPA) $-3 \mathrm{~km} \mathrm{~W}$ of Crucea, Allah Bair (Nature Reserve), 1.V.2009, leg. JK; Gura Dobrogei, 28-29.IV.2012, leg. KI, 26.V.2013, leg. KI, PA, RI, SzL, 7.VI.2018, leg. FK, KI, KJ, PA; Cheile Dobrogei, 2.V.2014, leg. KI, IZ, PA, SzL; Murfatlar, Fântâniţa, 4-5.VII.2014, leg. KI, OA, PA, SzL, 22.V.2015, leg. PA, RI, SzK, SzL, 7-8.VI.2018, leg. FK, KI, KJ, PA; Palazu Mic, 22.V.2015. leg. PA, RI, SzK, SzL; Băneasa, Canaraua Fetii, 23.V.2015, leg. PA, RI, SzK, SzL, 2-3.IV.2016, leg. FK, KI, PA, SzL; Cochirleni, 24.V.2015, leg. PA, RI, SzK, SzL; 23 August, 17.IX.2016, leg. FK, KI, KJ, PA; Limanu, 12.V.2017, leg. FK, KI, KJ, PA; Sinoie, Grindul Lupilor, 27.VI.2017, leg. KI, 5.VI.2018, leg. FK, KI, KJ, PA; 2 km from Sinoie to SE, 5.VI.2018, leg. FK,
KI, KJ, PA; Istria, Cetatea Histria, 6.VI.2018, leg. FK, KI, KJ, PA; Vadu, Grindul Chituc, 6.VI.2018, leg. KI; Crucea, 9.VI.2018, leg. KI.

Tanymecus (Tanymecus) palliatus (FABRICIUS, 1787) Published data: MonTANDON 1887, 1908; JaQUET 1900b, 1902b; FleCK 1905; NegRU 1968; TeOdOR 1993; TeOdor and Manole 1996; Raduta 2014

New data: Tulcea County: Greci, 22.VI.1963, leg. IS; Chilia Veche, 7.V.1964, leg. MW; Insula Popina, Lacul Razelm, 9.V.1992; Celic Dere, 20.V.1997, leg. RS; Babadag, 6.VI.2011, leg. IŞI (Coll. Mus. G. AntIPA) - Beştepe, 13.VI.1993, leg. TLA; Iancina, 11.VI.1993, leg. TLA; Atmagea, 28.IV.2012, leg. KI; Enisala, 24-25.V.2013, leg. KI, PA, RI, SzL, 29.IV3.V.2014, leg. KI, IZ, PA, SzL; Codru Monastery, 30.IV-3.V.2014, leg. KI, IZ, PA, SzL; Smârdan, Braţul Măcin, 7.V.2017, leg. FK, KI, KJ, PA; Nifon, pârâul Pârlita valley, 23.VI.2017, leg. KI, KJ, PA, TB; 2 km from Slava Rusă to NE, 3.VI.2018, leg. FK, KI, KJ, PA; Turcoaia, $6 \mathrm{~km}$ from Cerna to NW, 19.VI.2019, leg. KI, KJ, PA, TB; Constanţa County: Băneasa, Canaraua Fetii, 29.VI.1956, leg. NS, 11.VI.1964, leg. NS, 19.V.1993, leg. MB, 20.V.1993, leg. CH, 19.V.1994, leg. GA; Pădurea Băneasa, 31.V.1965, leg. ŞN; Furnica, Rezervaţia Dumbrăveni, 13-14.V.1995, leg. CP; Canton Silvic Furnica, Pădurea Dumbrăveni, 13.V.1995, leg. CP (Coll. Mus. G. ANTIPA) - Gura Dobrogei, 28-29.IV.2012, leg. KI, 26.V.2013, leg. KI, PA, RI, SzL; Murfatlar, Fântâniţa, 4-5.VII.2014, leg. KI, OA, PA, SzL; Băneasa, Canaraua Fetii (quarry), 2-3.IV.2016, leg. FK, KI, PA, SzL; Cochirleni, 3.IV.2016, leg. FK, KI, PA, SzL; Sinoie, Grindul Lupilor, 27.VI.2017, leg. KI.

Trachyphloeini GisTel, 1848

Cathormiocerus spinosus GoEZE, 1777

(= Trachyphloeus olivieri BEDEL, 1883)

New data: Tulcea County: Beştepe, 30.IV.1993, leg. TLA; Iancina, 2.V.1993, 11.VI.1993, leg. TLA.

\section{Pelletierellus turcicus (SEIDLITZ, 1868)}

(= Trachyphloeus turcicus SeIDLITZ, 1868)

Published data: Montandon 1908, Borovec 1992

New data: Tulcea County: Măcin, leg. ALM; Cerna, 26.V.2005, leg. IM; Măcin, Valea Suluc, 27.V.2005, leg. IM (Coll. Mus. G. AnTIPA) - Braţul Sfântu Gheorghe, 22.VI.2003, leg. TB; Măcin (hillside, 200 m), 29.V.2005, leg. CsS; Enisala, 24-25.V.2013, leg. KI, PA, RI, SzL; Greci (village), 8.V.2017, leg. FK, KI, KJ, PA; M-ţii Măcin, 5 km S Greci, 8.V.2017, leg. FK, KI, KJ, PA; Enisala (castle), 20.VI.2019, leg. KI, KJ, PA, TB; Jurilovca, 23.VI.2019, leg. KI, KJ, PA, TB; Constanţa County: Hârşova, leg. ALM (Coll. Mus. G. AntIPA) - Gura Dobrogei, 30.IV2.V.2014, leg. KI, IZ, PA, SzL, 12.V.2017, leg. FK, KI, KJ, PA, 7.VI.2018, leg. FK, KI, KJ, PA; Palazu Mic, 22.V.2015. leg. PA, RI, SzK, SzL; Cochirleni, 24.V.2015, leg. PA, RI, SzK, SzL; 2 km from Sinoie to 
SE, 27.VI.2017, leg. KI, KJ, PA, TB; Sinoie, Grindul Lupilor, 5.VI.2018, leg. FK, KI, KJ, PA.

Pelletierellus ventricosus (GERMAR, 1823)

(= Trachyphloeus ventricosus GERMAR, 1823)

New data: Tulcea County: Beştepe, 13.VI.1993, leg. TLA; Iancina, 11.VI.1993, leg. TLA; Slava Rusă, 1-3.V.2014, leg. KI, IZ, PA, SzL, 11.V.2017, leg. FK, KI, KJ, PA; Slava Rusă, Uspenia Monastery, 11.V.2017, leg. FK, KI, KJ, PA; 2,7 km from Horia to S, 28.VI.2017, leg. KI; 2 km from Slava Rusă to NE, 3.VI.2018, leg. FK, KI, KJ, PA; Codru Monastery, 4.VI.2018, leg. KI; Constanţa County: Băneasa, Canaraua Fetii, 23.V.2015, leg. PA, RI, SzK, SzL, 2-3.IV.2016, leg. FK, KI, PA, SzL; Gura Dobrogei, 12.V.2017, leg. FK, KI, KJ, PA.

Stuebenius amplithorax (FORMÁNEK, 1907)

(= Trachyphloeus amplithorax FoRMÁNEK, 1907)

Published data: MonTANDON 1908

\section{Trachyphloeus}

(Trachyphloeus)

alternans

GYLlenhal, 1834

Published data: Negru and Roşca 1967, Raduta 2014

New data: Tulcea County: M-ţii Măcin, Valea Fagilor (dried lake bed) 30.V.2005, leg. CsS; Slava Rusă, 1-3.V.2014, leg. KI, IZ, PA, SzL, 11.V.2017, leg. FK, KI, KJ, PA; Constanța County: Gura Dobrogei, 30.IV2.V.2014, leg. KI, IZ, PA, SzL; Murfatlar, Fântânița, 4-5.VII.2014, leg. KI, OA, PA, SzL, 22.V.2015. leg. PA, RI, SzK, SzL; Gura Dobrogei, 25, 27.VI.2017, leg. KI, KJ, PA, TB; Crucea, 9.VI.2018, leg. KI.

\section{Trachyphloeus}

GERMAR, 1823

(Trachyphloeus)

spinimanus

New data: Tulcea County: Iancina, 11.VI.1993, leg. TLA; Slava Rusă, 9.V.2017, leg. FK, KI, KJ, PA, 11.V.2017, leg. FK, KI, KJ, PA; Codru Monastery, 10-11.V.2017, leg. FK, KI, KJ, PA; Turcoaia, 6 km from Cerna to NW, 19.VI.2019, leg. KI, KJ, PA, TB; Constanţa County: Cheia env., Cheia Nature Reserve., 19.IX.2010, leg. JK; Gura Dobrogei, 30.IV-2.V.2014, leg. KI, IZ, PA, SzL, 12.V.2017, leg. FK, KI, KJ, PA, 7.VI.2018, leg. FK, KI, KJ, PA; Limanu, 12.V.2017, leg. FK, KI, KJ, PA.

Zarazagaia rugicollis SEIDLITZ, 1868

(= Trachyphloeus rugicollis SeIDLITZ, 1868)

Published data: JAQUeT 1899b, FleCK 1905

HYPERINAE MARSEUL, 1863

Hyperini MARSEUL, 1863

Brachypera (Antidonus) dauci (OLIVIER, 1807)

Published data: Montandon 1908, Negru and RoşCa 1967, RADUTA 2014

New data: Tulcea County: M-ţii Măcin, Dealurile Pricopanului, 14.VI.2007, leg. MC (Coll. Mus. R. ANGHELuŢǍ) - Canalul Magearu, 17.VI.1993, leg.
TLA; Babadag (forest), 20.V.2011, leg. CS; Enisala (castle), 30-31.III.2016, leg. FK, KI, PA, SzL, 13.IX.2016, leg. KI; Constanţa County: Istria env., Grindul Saele, Cetatea Histria, 22.IX.2010, leg. JK; Istria, Cetatea Histria, 14.IX.2016, leg. KI; Gura Dobrogei, 25.VI.2017, leg. KI, KJ, PA, TB.

\section{Brachypera (Antidonus) zoilus (SCOPOLI, 1763)}

Published data: MonTANDON 1887, 1908; FleCK 1905; Negru and RoşCa 1967; Raduta 2014

New data: Tulcea County: Codru Monastery, 30-31. III.2016, leg. FK, KI, PA, SzL; M-ţii Măcin, 5 km S Greci, 8.V.2017, leg. FK, KI, KJ, PA; Constanţa County: Mangalia, leg. ALM (Coll. Mus. G. Antipa) - Balta Limanu, 5-6.VII.2014, leg. KI, OA, PA, SzL.

Coniatus (Bagoides) splendidulus (FABRICIUS, 1781) Published data: Negru 1968, Ieniştea 1974, TeOdor 1993, TeOdor and Manole 1996, Raduta 2014 New data: Tulcea County: Sulina, 20-23.VII.1964, leg. ŞN, 21.VII.1964, leg. APG, 30.IX.1968, leg. MW, 18.VI.1969, leg. ŞN, 09-12.VII.1969, leg. AtR; Pădurea Caraorman, Delta Dunării, 24.VI.1991, leg. PG (Coll. Mus. G. ANTIPA) - Caraorman, 4-14. VII.1991, leg. AR; Maliuc, Mila 26, 21.VI.1993, leg. TLA; Pădurea Letea (forest), 17.VI.1993, leg. TLA; Jijila, râul Jijila, 23.VI.2017, leg. KI, KJ, PA, TB, 19.VI.2019, leg. KI, KJ, PA, TB.

\section{Donus tessellatus (BoHeman, 1834)}

New data: Tulcea County: Nifon, pârâul Pârlita valley, 23.VI.2017, leg. KI, KJ, PA, TB.

\section{Hypera (Boreohypera) diversipunctata (SCHRANK, 1798)}

New data: Tulcea County: Enisala, Cetatea Heraclea, 25.VI.2017, leg. KI, KJ, PA, TB; Nifon, pârâul Pârlita valley, 23.VI.2017, leg. KI; Constanţa County: Băneasa, Canaraua Fetei, 7.VI.2008, leg. GP (Coll. Mus. R. ANGHELUŢă).

\section{Hypera (Dapalinus) meles (FABRICIUS, 1792)}

Published data: MonTANDON 1908

New data: Tulcea County: M-ţii Măcin,Valea lui Arsu, 6.VI.2005, leg. MC (Coll. Mus. R. ANGHELUȚă); Măcin, 10.V.2011, leg. IȘI (Coll. Mus. G. ANTIPA) - Măcin (hillside, 200 m), 29.V.2005, leg. CsS; M-ţii Măcin, Valea Fagilor (dried lake bed), 30.V.2005, leg. CsS; M-ţii Măcin,Valea Fagilor (quarry), 30.V.2005, leg. CsS; Frecăţei, Celic Dere Monastery (hillside) 31.V.2005, leg. CsS; M-ţii Măcin, Pricopan, 1.VI.2005, leg. CsS; M-ţii Măcin, to the Peak Ţuțuiatu, 28.IV.2010, leg. TLA; Slava Rusă, 9.V.2017, leg. FK, KI, KJ, PA; M-ţii Măcin, 5 km S Greci, 8.V.2017, leg. FK, KI, KJ, PA; Slava Rusă, Uspenia Monastery, 11.V.2017, leg. FK, KI, KJ, PA; N from Nifon, 23.VI.2017, leg. KI, KJ, PA, TB; Smârdan, Braţul Măcin, 22.VI.2017, leg. KI, KJ, PA, TB; Greci (village), 22.VI.2017, leg. KI; Codru 
Monastery, 10.V.2017, leg. KI; 1,5 km from Bediaud to SW, 25.VI.2017, leg. KI; Constanța County: Esechioi (forest), 7.VII.2014, leg. KI, OA, PA, SzL; Hagieni, Pădurea Hagieni (forest), 17.IX.2016, leg. FK, KI, KJ, PA; Gura Dobrogei, 12.V.2017, leg. FK, KI, KJ, PA; Albeşti, Pădurea Hagieni (forest), 12.V.2017, leg. FK, KI, KJ, PA.

\section{Hypera (Dapalinus) striata (BOHEMAN, 1834)}

New data: Tulcea County: Slava Rusă, 1-3.V.2014, leg. KI, IZ, PA, SzL; Constanţa County: Esechioi (forest), 7.VII.2014, leg. KI, OA, PA, SzL.

\section{Hypera (Eririnomorphus) arundinis (PAYKULL, 1792) \\ Published data: Teodor and Manole 1996, Raduta 2014 \\ New data: Tulcea County: Canalul Magearu, 16.VI.1993, leg. TLA.}

Hypera (Eririnomorphus) conmaculata (HERBST, 1795) $(=$ adspersa FABRICIUS, 1792; = pollux FABRICIUS, 1801)

Published data: MonTANDON 1908

New data: Tulcea County: Delta Dunării, Caraorman, 13.VI.2001, leg. CR (Coll. Mus. R. Angheluță); C. A. Rosetti, VII.1950, leg. IC (Coll. Mus. G. ANTIPA) Smârdan, Braţul Măcin, 22.VI.2017, leg. KI, KJ, PA, TB.

\section{Hypera (Eririnomorphus) rumicis (LINNÉ, 1758)}

Published data: TeOdor and Manole 1996, Raduta 2014

New data: Tulcea County: Delta Dunării, Caraorman, 13.VI.2001, leg. CR (Coll. Mus. R. Angheluță); Chilia, 7.V.1964, leg. IS; Periprava, Delta, 4.VII.1965, leg. ŞN; Delta Dunării, Sfântu Gheorghe, 12-13. VI.1995, leg. VB (Coll. Mus. G. ANTIPA) - Doloşman, 11.V.1992, leg. TLA; Smârdan, Braţul Măcin, 7.V.2017, leg. FK, KI, KJ, PA; 2 km from Slava Rusă to NE, 3.VI.2018, leg. KI; Sinoie, 21, 23.VI.2019, leg. KI, KJ, PA, TB; Constanţa County: Limanu, Pădurea Hagieni, 22.V.1993, leg. CP (Coll. Mus. G. ANTIPA) - Băneasa, Canaraua Fetii (quarry), 2-3. IV.2016, leg. FK, KI, PA, SzL; Băneasa, Canaraua Fetii (forest), 15-17.IX.2016, leg. KI, KJ, PA, TB; 2 $\mathrm{km}$ from Sinoie to SE, 27.VI.2017, leg. KI.

\section{Hypera (Hypera) melancholica (FABRICIUS, 1792)}

(= murina FABRICIUS, 1792; = fuscocinerea MARSHAM, 1802)

New data: Tulcea County: Beştepe, 13-14.VI.1993, leg. IM (Coll. Mus. G. ANTIPA) - Doloşman, 11.V.1992, leg. TLA; W of Horia, 6.V.2009, leg. JK; Constanţa County: Oltina, Canaraua Fetii, 14.IV.1958, leg. APG; Hagieni, 6.VI.1964, leg. NS; Agigea, 23.VI.1964, leg. IC (Coll. Mus. G. ANTIPA).

Hypera (Hypera) miles (PAYKULL, 1792) (= pedestris
PAYKull, 1792; = suspiciosa HerbSt, 1795)

New data: Tulcea County: Smârdan, Braţul Măcin, 7.V.2017, leg. FK, KI, KJ, PA.

Hypera (Hypera) nigrirostris (FABRICIUS, 1775)

Published data: TeOdor and Manole 1996, Raduta 2014

New data: Tulcea County: Canalul Magearu, 17.VI.1993, leg. TLA; Pădurea Letea (forest), 17.VI.1993, leg. TLA; Sulina, 4.VII.2005, leg. DDC; Smârdan, Braţul Măcin, 28.VI.2017, leg. KI, KJ, PA, TB; Constanţa County: Băneasa, Canaraua Fetii (quarry), 2-3.IV.2016, leg. FK, KI, PA, SzL.

Hypera (Hypera) ononidis (CHEVROLAT, 1863)

Published data: Teodor and Manole 1996; Raduta 2014

Hypera (Hypera) plantaginis (DEGEER, 1775)

New data: Tulcea County: Beştepe, 30.IV.1993, 13 -14.VI.1993, leg. TLA; Constanţa County: Esechioi (forest), 7.VII.2014, leg. KI, OA, PA, SzL.

\section{Hypera (Hypera) postica (GYLLENHAL, 1813)}

Published data: Montandon 1887, 1908; JaQUet 1899a; FleCK 1905; NEGRU and RoşCA 1967; RADUTA 2014

New data: Tulcea County: M-ţii Măcin, Dealurile Pricopanului, 14.VI.2007, leg. MC; M-ții Măcin, Valea lui Moş Kalâm, 5.X.2007, leg. CI (Coll. Mus. R. Angheluță); Periprava, Letea, 13.V.1964, leg. XS (Coll. Mus. G. ANTIPA) - Beştepe, 30.IV.1993, leg. TLA; Măcin (hillside, 200 m), 29.V.2005, leg. CsS; M-ţii Măcin, Valea Fagilor (quarry), 30.V.2005, leg. CsS; Babadag (forest), 2.VI.2005, leg. CsS; Codru env., 3.V.2009, leg. JK, 5.V.2009, leg. JK; M-ţii Măcin, Greci env., 6.V.2009, leg. JK; M-ţii Măcin, Hamcearca, 6.V.2009, leg. JK; M-ţii Măcin, to the Peak Ţuţuiatu, 28.IV.2010, leg. TLA; Enisala, 2425.V.2013, leg. KI, PA, RI, SzL, 29.IV-3.V.2014, leg. KI, IZ, PA, SzL; Slava Rusă, 31.III.2016, leg. FK, KI, PA, SzL; Enisala (forest), 13.IX.2016, leg. KI, KJ, PA, TB, 20.VI.2019, leg. KI, KJ, PA, TB; Nifon, pârâul Pârlita valley, 23.VI.2017, leg. KI, KJ, PA, TB; Codru Monastery, 24-25.VI.2017, leg. KI, KJ, PA, TB; 2,7 km from Horia to S, 28.VI.2017, leg. KI, KJ, PA, TB; 2 km from Slava Rusă to NE, 3.VI.2018, leg. FK, KI, KJ, PA; Turcoaia, $6 \mathrm{~km}$ from Cerna to NW, 19.VI.2019, leg. KI, KJ, PA, TB; Enisala (castle), 20.VI.2019, leg. KI, KJ, PA, TB; M-ţii Măcin, Greci, 18-19.VI.2019, leg. KI; Constanţa County: Valul lui Traian, 26.V.1955, 19.VI.1955, leg. XS; Agigea, 20.VII.1964, leg. MW; Negureni, 25.VI.1995, leg. CP; Cotu Văii, 23.VII.1997, leg. CP (Coll. Mus. G. ANTIPA) - Cheia env., Cheia Nature Reserve., 2.V.2009, leg. JK; Istria env., Cetatea Histria, 2-4. V.2009, leg. JK; Gura Dobrogei, 30.IV-2.V.2014, leg. KI, IZ, PA, SzL, 12.V.2017, leg. FK, KI, KJ, PA, 25-27.VI.2017, leg. KI, KJ, PA, TB, 7.VI.2018, leg. FK, KI, KJ, PA; 
Murfatlar, Fântâniţa, 4-5.VII.2014, leg. KI, OA, PA, SzL, 22.V.2015, leg. PA, RI, SzK, SzL, 7-8.VI.2018, leg. FK, KI, KJ, PA; Esechioi (forest), 7.VII.2014, leg. KI, OA, PA, SzL; Băneasa, Canaraua Fetii (quarry), 7.VII.2014, leg. KI, OA, PA, SzL, 23.V.2015, leg. PA, RI, SzK, SzL, 2-3.IV.2016, leg. FK, KI, PA, SzL; Negru Vodă (Nature Reserve) 25.IX.2015, leg. KI, KJ, PA; Albeşti, Pădurea Hagieni (forest), 12.V.2017, leg. FK, KI, KJ, PA; Istria, Cetatea Histria, 26-28.VI.2017, leg. KI, KJ, PA, TB, 11.V.2017, leg. FK, KI, KJ, PA, 6.VI.2018, leg. FK, KI, KJ, PA, 21.VI.2019, leg. KI, KJ, PA, TB; Costineşti (coast), 17.IX.2016, leg. FK, KI, KJ, PA; Vadu, Grindul Chituc, 6.VI.2018, leg. FK, KI, KJ, PA; Sinoie, Grindul Lupilor, 5.VI.2018, leg. FK, KI, KJ, PA, 22.VI.2019, leg. KI, KJ, PA, TB; Crucea, 9.VI.2018, leg. FK, KI, KJ, PA.

Hypera (Hypera) viciae (GYLLENHAL, 1813)

Published data: JAQUET 1899b, FleCK 1905

Hypera (Kippenbergia) arator (LINNÉ, 1758)

Published data: MonTANDON 1908

New data: Tulcea County: M-ţii Măcin, Chiscura Chelului (Parcul Naţional M-ţii Măcin), 13.VI.2009, leg. CŞ (Coll. Mus. R. ANGHELuŢă) - Pădurea Letea (forest), 17.VI.1993, leg. TLA; Codru Monastery, 23-25.V.2013, leg. KI, PA, RI, SzL; Enisala, 2425.V.2013, leg. KI, PA, RI, SzL; Enisalai (castle), 21.V.2015, leg. PA, RI, SzK, SzL; M-ţii Măcin, 5 km S Greci, 8.V.2017, leg. KI; Constanţa County: Gura Dobrogei, 7.VI.2018, leg. FK, KI, KJ. PA.

\section{Limobius borealis borealis (PAYKULL, 1792)}

Published data: MontANDON 1887, 1908; FLECK 1905 New data: Tulcea County: Greci, 22.VI.1963, leg. IS; Măcin, Valea Suluc, 27.V.2005, leg. IM (Coll. Mus. G. Antipa) - M-ţii Măcin, Valea Fagilor (quarry) 30.V.2005, leg. CsS; W of Horia, 6.V.2009, leg. JK; Codru Monastery, 23-25.V.2013, leg. KI, PA, RI, SzL; Enisala, 24-25.V.2013, leg. KI, PA, RI, SzL; Slava Rusă, 1-3.V.2014, leg. KI, IZ, PA, SzL, Slava Rusă, 11.V.2017, leg. FK, KI, KJ, PA; Enisala (castle), 21.V.2015, leg. PA, RI, SzK, SzL, 22.IX.2015, leg. KI, KJ, PA, 10.V.2017, leg. FK, KI, KJ, PA; M-ţii Măcin, 5 km S Greci, 8.V.2017, leg. FK, KI, KJ, PA; M-ţii Măcin, Greci, 8.V.2017, leg. FK, KI, KJ, PA, 18-19. VI.2019, leg. KI; Smârdan, Braţul Măcin, 22.VI.2017, leg. KI, KJ, PA, TB; Turcoaia, $6 \mathrm{~km}$ from Cerna to NW, 24.VI.2017, leg. KI, KJ, PA, TB, 19.VI.2019, leg. KI, KJ, PA, TB; $1,5 \mathrm{~km}$ from Beidaud to SW, 25.VI.2017, leg. KI, KJ, PA, TB; Enisala (wooded pasture), 4.VI.2018, leg. FK, KI, KJ, PA; Constanţa County: Agigea, 23.VI.1960, leg. MW (Coll. Mus. G. Antipa) - Gura Dobrogei, 28-29.IV.2012, leg. KI, 30.IV-2.V.2014, leg. KI, IZ, PA, SzL, 1.IV.2016, leg. FK, KI, PA, SzL, 7.VI.2018, leg. FK, KI, KJ, PA; Palazu Mic, 22.V.2015, leg. PA, RI, SzK, SzL; Sinoie, Grindul Lupilor, 5.VI.2018, leg. FK, KI, KJ, PA.
Pachypera kraatzi (CAPIOMONT, 1868)

Published data: Montandon 1908, Negru and RoşCa 1967, RADUTA 2014

New data: Constanţa County: Murfatlar, X.1951, leg. NS (Coll. Mus. G. ANTIPA).

\section{LIXINAE SCHÖNHERR, 1823}

Cleonini SCHÖNHERR, 1826

Asproparthenis albicans GYLLENHAL, 1834

New data: Tulcea County: Sarinasuf, 24.VI.1962, leg. XS (Coll. Mus. G. AnTIPA).

\section{Asproparthenis carinata ZoUBKOFF, 1829 \\ Published data: MonTANDON 1887 \\ New data: Tulcea County: Sarinasuf, 24.VI.1962, leg. XS (Coll. Mus. G. ANTIPA); Constanţa County: Eforie Sud, Carmen Sylva, 22.VI.1952, leg. NS (Coll. Mus. G. ANTIPA).}

[Asproparthenis lusca (ChEVROLAT, 1869)]

Published data: Negru and RoşCa 1967, Raduta 2014 - Spread: Western Europe. Incorrect identification.

\section{Asproparthenis punctiventris (GERMAR, 1823)}

Published data: JAQUET 1898a, 1900c; FLeCK 1905; Montandon 1908; Negru 1968; Ciochia 1985; TeOdor 1993; TeOdor and Manole 1996; Raduta 2014

New data: Tulcea County: - Țuţuiatu, PNMM (467 m), 12.V.2010 (Coll. Mus. R. ANGHELuȚă); Sarinasuf, 24.VI.1962, leg. XS; C. A. Rosetti, 20.VII.1964, leg. XS; Pădurea Babadag, 12.V.1982. (Coll. Mus. G. ANTIPA) - Beştepe, 13.VI.1993, leg. TLA; Iancina, 11.VI.1993, leg. TLA; Braţul Sfântu Gheorghe, 21-22. VI.2003, leg. TB; M-ţii Măcin, to the Peak Ţuţuiatu, 28.IV.2010, leg. TLA; Slava Rusă, 1-3.V.2014, leg. KI, IZ, PA, SzL; Constanţa County: Hârşova, leg. ALM; Oltina, Păd. Ciufâtu, 24.IV.1956, leg. APG; Oltina, Canaraua Fetii, 13.V.1958, leg. APG; Agigea, 22.VI.1964, leg. MW; Băneasa, Canaraua Fetii, 17.VII.1964, leg. NS, 21.VII.1993, leg. GA; Negureni, Pădurea Negureni, 21.VII.1993, leg. GA; Pădurea Dumbrăveni, 19.V.1994, leg. CP; Furnica, Pădurea Dumbrăveni, 14.V.1995, leg. GA (Coll. Mus. G. AnTIPA) - Cheile Dobrogei, 2.V.2014, leg. KI, IZ, PA, SzL; $2 \mathrm{~km}$ from Sinoie to SE, 27.VI.2017, leg. KI, KJ, PA, TB; Istria, Cetatea Histria, 6.VI.2018, leg. FK, KI, KJ, PA, 21.VI.2019, leg. TB.

\section{Asproparthenis vexata (GYLLENHAL, 1834)}

(= Bothynoderes vexatus GyLLENHAL, 1834)

New data: Tulcea County: Caraorman, 12. V.1968, leg. XP (Coll. Mus. G. AnTIPA); Constanţa County: Băneasa, Canaraua Fetii, 17.VII.1964, leg. NS (Coll. Mus. G. AnTIPA) - $3 \mathrm{~km} \mathrm{~W}$ of Crucea, Allah Bair (Nature Reserve), 1.V.2009, leg. JK.

Bothynoderes affinis (SCHRANK, 1781) (= Curculio fasciatus O. F. MüLLER, 1776) 
Published data: Montandon 1887, 1906, 1908; Fleck 1905; TeOdor and Manole 1996; Raduta 2014

New data: Tulcea County: Delta Dunării, canal Babarada-Şontea, 30.VII.2004, leg. GP (Coll. Mus. R. ANGHELUŢĂ).

Bothynoderes declivis (OLIVIER, 1807)

Published data: MonTANDON 1908

New data: Tulcea County: Pădurea Letea $(1,5 \mathrm{~m})$, 27.VII.2004, leg. GP (Coll. Mus. R. Angheluță) Caraorman, 14.V.1992, leg. TLA.

Chromonotus vittatus (ZouBKofF, 1829)

Published data: Montandon 1908, Negru and Roşca 1967

Cleonis pigra (SCOPOLI, 1763)

Published data: Fleck 1905, Teodor 1993, Teodor and Manole 1996, Raduta 2014

New data: Tulcea County: Luncaviţa, Valea Fagilor, 23.V.1997, leg. CP, RS (Coll. Mus. G. ANTIPA) Smârdan, Braţul Măcin, 21-23.IX.2015, leg. KI, KJ, PA; M-ţii Măcin, Greci, 18-19.VI.2019, leg. KI, KJ, PA, TB; Constanţa County: Mangalia, leg. ALM; Hagieni, 29.VI.1963, leg. NS; Dumbrăveni, 2.IX.1964, leg. NS; Bugeac, 20.V.1965, leg. NS; Canaraua Fetii, 1.X.1965, leg. ŞN; Furnica, Pădurea Dumbrăveni, 13.V.1995, leg. MA (Coll. Mus. G. ANTIPA) $-3 \mathrm{~km} \mathrm{~W}$ of Crucea, Allah Bair (Nature Reserve), 18.IX.2010, leg. JK; Gura Dobrogei, 2829.IV.2012, leg. KI, 30.IV-2.V.2014, leg. KI, IZ, PA, SzL; Ostrov, Lacul Bugeac, 16.IX.2016, leg. KI, KJ, PA, TB; 23 August, 17.IX.2016, leg. FK, KI, KJ, PA.

\section{Coniocleonus (Augustecleonus) nebulosus (LINNÉ,} 1758)

Published data: Ieniştea and Negru 1956, Negru and RoşCa 1967, RaDUTA 2014

Coniocleonus (Plagiographus) cicatricosus (НОРРЕ, 1795)

Published data: Negru and RoşCa 1967

\section{Coniocleonus (Plagiographus) nigrosuturatus}

(GoEZE, 1777)

Published data: Montandon 1908, Negru 1957, Negru and RoşCa 1967, Raduta 2014

New data: Constanţa County: Ostrov, Esechioi, 14.V.1963, leg. NS; Băneasa, Canaraua Fetii, 27.IV.1963, leg. NS, 1.X.1965, leg. ŞN; Hagieni, 28.VIII.1964, leg. NS (Coll. Mus. G. ANTIPA).

Coniocleonus (Plagiographus) pseudobliquus (J. MüLLER, 1921)

Published data: SKuHrovec et al. 2014

New data: Tulcea County: M-ţii Măcin, Cetatea (Parcul Naţional M-ţii Măcin, 301 m), 26.VIII.2008, leg. CŞ (Coll. Mus. R. Angheluţă); M-ţii Măcin,
Greci, 11.VII.1960, leg. IS; Babadag, 3.X.1963, leg. XS, 31.X.1963, leg. AMa, 23.V.1966., leg. NS; Letea, 13.IV.2011, leg. IŞI (Coll. Mus. G. ANTIPA) - Ciucurova env., 4.V.2009, leg. JK; Stejaru env., 4-5.V.2009, leg. JK; Codru env., 5.V.2009, leg. JK; M-ţii Măcin, Hamcearca, 6.V.2009, leg. JK; Greci env., 6.V.2009, leg. JK; M-ţii Măcin, to the Peak Ţuţuiatu, 28.IV.2010, leg. TLA, AR; Enisala, 2425.V.2013, leg. KI, PA, RI, SzL, 30-31.III.2016, leg. FK, KI, PA, SzL; Constanţa County: Pădurea Comorova, 11-12.IX.1959, leg. ŞN (Coll. Mus. G. AnTIPA) - Vama Veche, 13.V.2017, leg. KI.

\section{Cyphocleonus achates FÅHRAEUS, 1842}

New data: Constanţa County: Murfatlar, 24.IX.1951, leg. NS (Coll. Mus. G. ANTIPA).

Cyphocleonus dealbatus (GMELIN, 1790) (= Curculio tigrinus PANZER, 1789)

Published data: Montandon 1887, 1908; FLECK 1905; RADUTA 2014

New data: Tulcea County: Dinogeţia, 28.VII.2001, 23.V.2002, leg. CŞ; M-ţii Măcin, Cetatea (Parcul Naţional M-ţii Măcin, 301 m), 26.VIII.2008, leg. CŞ (Coll. Mus. R. Angheluță); Teliţa, Mănăstirea Celic Dere, 20.V.1997, leg. RS (Coll. Mus. G. ANTIPA) Parcheş 23.IX.2015, leg. KI, KJ, PA.

Leucophyes pedestris (PODA, 1761)

Published data: FleCK 1905, MonTANDON 1908

Maximus strabus (GYLLENHAL, 1834) (= Temnorhinus strabus GYLLENHAL, 1834)

Published data: Montandon 1908

New data: Tulcea County: Sarinasuf, 24.VI.1962, leg. XS (Coll. Mus. G. ANTIPA).

Mecaspis alternans (HERBST, 1795)

Published data: MonTANDON 1908

New data: Tulcea County: Codru Monastery, 1011.V.2017, leg. FK, KI, KJ, PA.

Mecaspis incisurata (GYLLENHAL, 1834)

Published data: MonTANDON 1908

Mecaspis striatella (FABRICIUS, 1792)

Published data: JaQUet 1899b, FleCK 1905

Pachycerus madidus (OLIVIER, 1807)

Published data: JAQUET 1900b

Pachycerus segnis (GERMAR, 1823) (= cordiger Germar, 1819)

Published data: Fleck 1905, Montandon 1908

New data: Constanţa County: Esechioi, 13-14.V.1963, leg. NS; Băneasa, Canaraua Fetii, 17-19.VII.1964, leg. NS (Coll. Mus. G. ANTIPA).

Pleurocleonus quadrivittatus (ZoUBKOFF, 1829) 
Published data: RADUTA 2014

New data: Tulcea County: Măcin, leg. ALM (Coll. Mus. G. AntiPA); Constanţa County: Oltina, 15.V.1955, leg. NS (Coll. Mus. G. ANTIPA).

\section{[Pseudocleonus (Pseudocleonus) carinatus (Gyllenhal, 1834)]}

Published data: MonTANDON 1887 - Spread: Western Europe. Incorrect identification.

\section{Pseudocleonus (Pseudocleonus) cinereus (SCHRANK, 1781)}

Published data: Montandon 1908, Negru and RoşCa 1967, RADUTA 2014

New data: Tulcea County: Măcin, ALM; Babadag, 22.VI.1956, leg. NS (Coll. Mus. G. ANTIPA) - Insula Popina, 9.V.1992, leg. TLA; Iancina, 11.VI.1993, leg. TLA; Constanţa County: Băneasa, Canaraua Fetii, 11.VI.1964, leg. NS; Băneasa, 17-20.VII.1964., leg. NS (Coll. Mus. G. Antipa).

\section{*Rhabdorrhynchus echii BRAHM, 1790}

New data: Tulcea County: Periprava, Delta, 1.VII.1965, leg. ŞN, 14.VII.1966, leg. XS (Coll. Mus. G. ANTIPA).

\section{Stephanocleonus (Sanzia) microgrammus (Gyllenhal, 1834) \\ Published data: Montandon 1908}

Temnorhinus (Temnorhinus) mixtus (FABRICIUS, 1792) (= Conorhynchus (Pycnodactylus) conicirostris OLIVIER, 1807)

Published data: FLECK 1905

Lixini SCHÖNHERR, 1823

Bangasternus fausti (REITTER, 1890)

Published data: Osella and Magnano 1984, Colonnelli and Whitehead 1990

New data: Tulcea County: Cerna, 26.V.2005, leg. IM (Coll. Mus. G. AntIPA).

\section{Bangasternus orientalis (CAPIOMONT, 1873)}

Published data: JAQUET 1899b, FLECK 1905, Montandon 1908, Colonnelli and WhitehEAD 1990

New data: Tulcea County: Celic Dere, 24.V.1997, leg. CP (Coll. Mus. G. ANTIPA) - Agighiol, 12.VI.1993, leg. TLA; Enisala, 24-25.V.2013, leg. KI, PA, RI, SzL, 29.IV-3.V.2014, leg. KI, IZ, PA, SzL; N from Nifon, 23.VI.2017, leg. KI, KJ, PA, TB; S from Hamcearca, 24.VI.2017, leg. TB; Turcoaia, 6 km from Cerna to NW, 3.VI.2018, leg. FK, KI, KJ, PA; Smârdan, Braţul Măcin, 18.VI.2019, leg. KI, KJ, PA, TB; Constanţa County: Gura Dobrogei, 26.V.2013, leg. KI, PA, RI, SzL, 25-27.VI.2017, leg. KI; Murfatlar, Fântâniţa, 4-5.VII.2014, leg. KI, OA, PA, SzL; Balta Limanu, 5-6.VII.2014, leg. KI, OA, PA, SzL; Esechioi (forest), 7.VII.2014, leg. KI, OA,

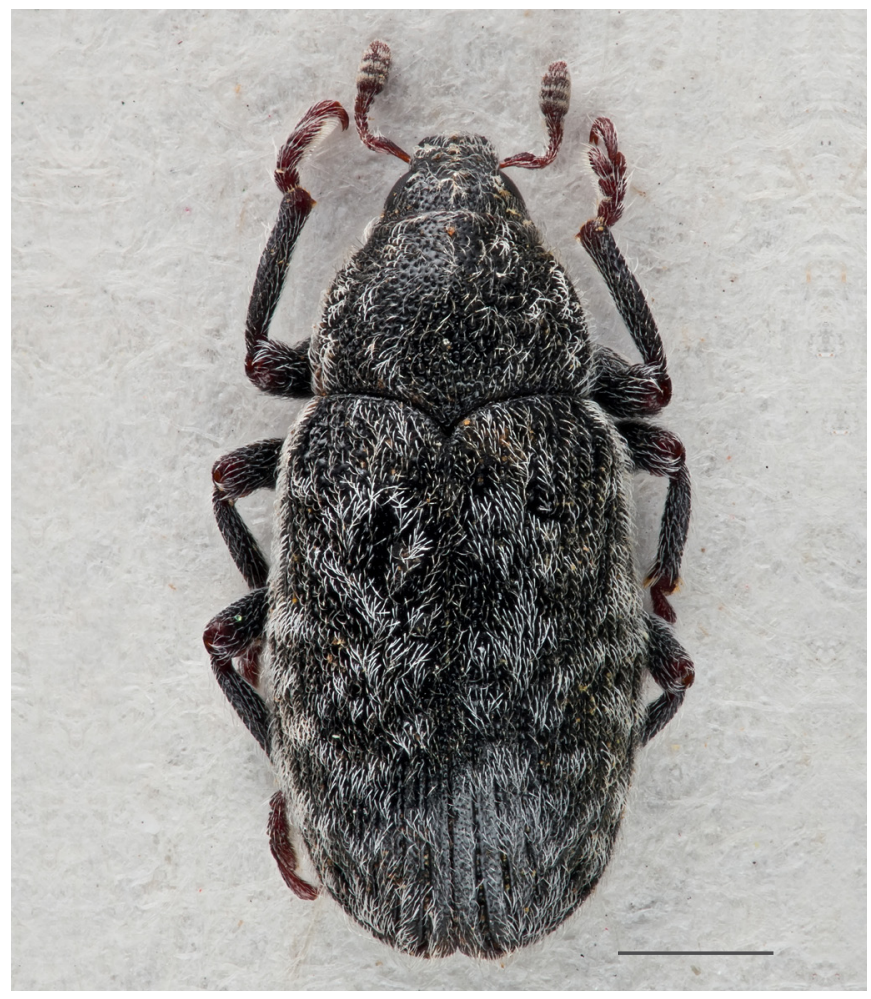

Fig. 23. Bangasternus planifrons (BRULlé, 1832). Scale bar: $1 \mathrm{~mm}$. Photo Zoltán Csata

PA, SzL; Cochirleni, 8.VII.2014, leg. KI, OA, PA, SzL; Palazu Mic, 22.V.2015, leg. PA, RI, SzK, SzL; Băneasa, Canaraua Fetii, 23.V.2015, leg. PA, RI, SzK, SzL.

*Bangasternus planifrons (BRULLÉ, 1832) (fig. 23) New data: Constanţa County: Balta Limanu, 5-6. VII.2014, leg. KI, OA, PA, SzL.

\section{Lachnaeus crinitus SCHÖNHERR, 1836}

Published data: MonTANDON 1887

New data: Tulcea County: Babadag, Visterna, 17.V.1963, leg. IC; Babadag, Valea Caugagiei, 17.V.1963, leg. IC (Coll. Mus. G. ANTIPA) - Atmagea env., 24.IX.2010, leg. JK; Smârdan, Braţul Măcin, 22.VI.2017, leg. KI, KJ, PA, TB; Greci (village), 22.VI.2017, leg. KI, KJ, PA, TB; Enisala, Cetatea Heraclea, 25.VI.2017, leg. KI.

\section{*Larinus (Larinomesius) atomarius CAPIOMONT, 1874}

New data: Constanţa County: Esechioi (forest), 7.VII.2014, leg. KI, OA, PA, SzL.

*Larinus (Larinomesius) canescens GyllENHAL, 1835 (fig. 24)

New data: Tulcea County: Codru Monastery, 2325.V.2013, leg. KI, PA, RI, SzL, 20.VI.2019, leg. KI, KJ, PA, TB; Constanța County: Pădurea Hagieni, 19.VI.1962, leg. AR; Băneasa, Canaraua Fetii, 16-19. VI.1964.; Limanu, Pădurea Hagieni, 11.VII.1992, leg. RS; Hagieni, 17-22.VII.1997, leg. CP (Coll. Mus. G. ANTIPA) - Murfatlar, Fântâniţa, 4-5.VII.2014, leg. 


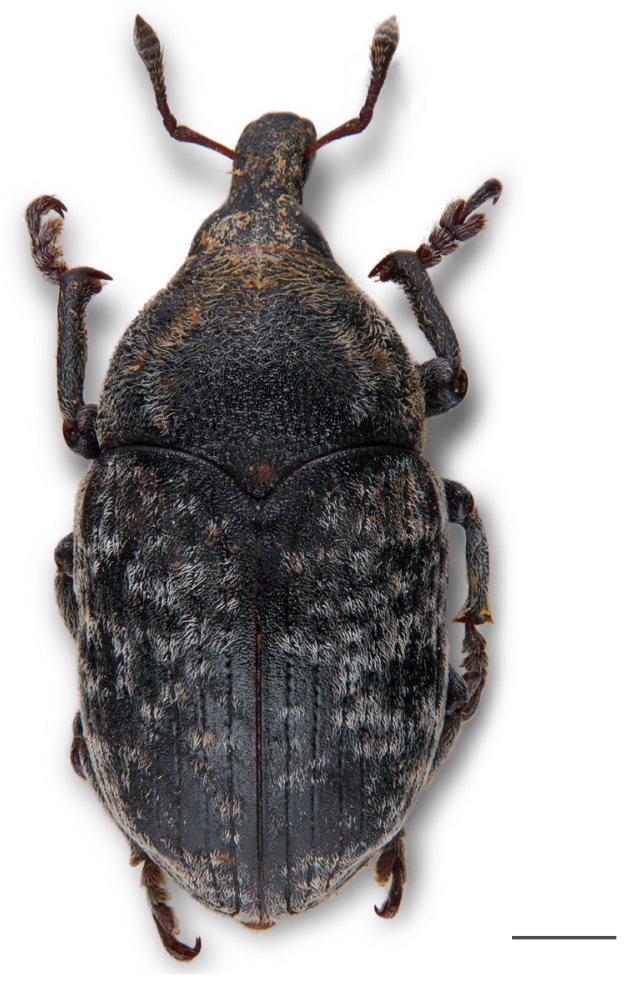

Fig. 24. Larinus (Larinomesius) canescens GyllenHAL, 1835. Scale bar: $1 \mathrm{~mm}$. Photo Zoltán CsatA

KI, OA, PA, SzL, 7-8.VI.2018, leg. FK, KI, KJ, PA; Gura Dobrogei, 25-27.VI.2017, leg. KI, KJ, PA, TB, 7.VI.2018, leg. FK, KI, KJ, PA; Crucea, 9.VI.2018, leg. KI.

Larinus (Larinomesius) minutus GYLLENHAL, 1835 Published data: JAQUET 1899a, 1899b; FLECK 1905; Negru and Roşca 1967; Raduta 2014

New data: Tulcea County: Caraorman, 19.VII.1960, leg. IS, 21.IX.1967., 3.VII.1968, leg. MW, 21-26. IX.1967, leg. ACa, 21.VII.1979, leg. APG; C. A. Rosetti, 20.VII.1964, leg. ŞN XS, 26.V.1980, leg. APG; Pădurea Caraorman, 24.VI.1991., 15.V.1992, leg. NG, 24.VIII.1992, leg. AP (Coll. Mus. G. ANTIPA) - Braţul Sfầntu Gheorghe, 21-22.VI.2003, leg. TB; Codru Monastery, 23-25.V.2013, leg. KI, PA, RI, SzL; Enisala, 24-25.V.2013, leg. KI, PA, RI, SzL; Enisala, Cetatea Heraclea, 25.VI.2017, leg. KI, KJ, PA, TB; Greci (village), 22.VI.2017, leg. KI, KJ, PA, TB; Turcoaia, $6 \mathrm{~km}$ from Cerna to NW, 3.VI.2018, leg. FK, KI, KJ, PA; 2 km from Slava Rusă to NE, 3.VI.2018, leg. FK, KI, KJ, PA; Enisala (castle), 20.VI.2019, leg. KI, KJ, PA, TB; Jurilovca, 23.VI.2019, leg. KI, KJ, PA, TB; M-ţii Măcin, Greci, 18-19.VI.2019, leg. KI; Constanţa County: Mangalia Nord, Staţiunea Olimp, 19-20.VII.2003, leg. RS (Coll. Mus. G. ANTIPA) Murfatlar, Fântâniţa, 4-5.VII.2014, leg. KI, OA, PA, SzL; Balta Limanu, 5-6.VII.2014, leg. KI, OA, PA, SzL; Cochirleni, 8.VII.2014, leg. KI, OA, PA, SzL; Sinoie, Grindul Lupilor, 27.VI.2017, leg. TB, 5.VI.2018, leg. FK, KI, KJ, PA, 22.VI.2019, leg. KI, KJ, PA, TB; Vadu, Grindul Chituc, 22.VI.2019, leg. KI, KJ, PA, TB.

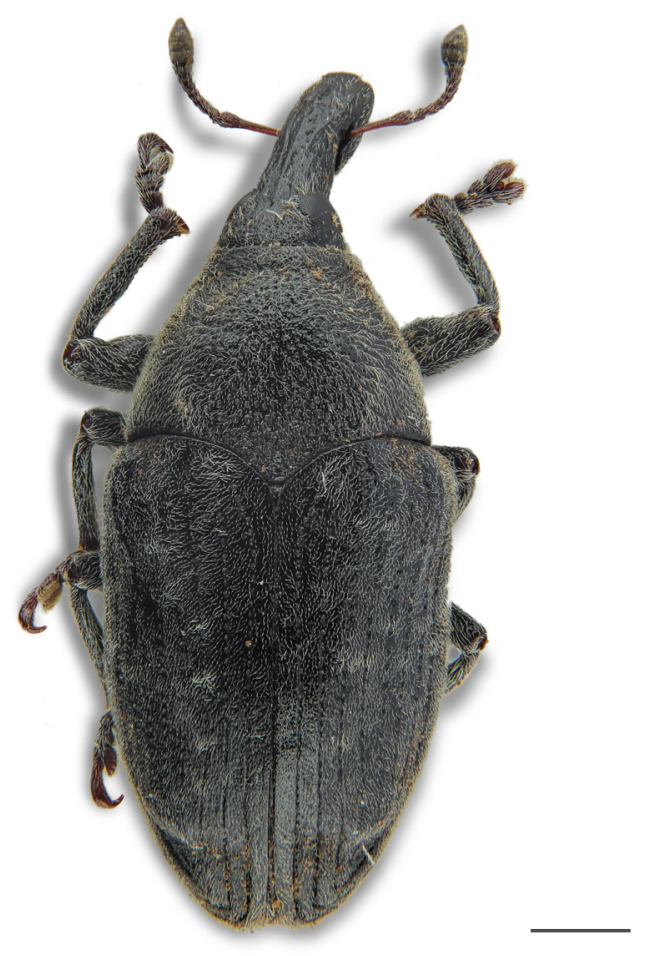

Fig. 25. Larinus (Larinomesius) syriacus GyllEnHAL, 1835. Scale bar: $1 \mathrm{~mm}$. Photo Zoltán CsatA

Larinus (Larinomesius) obtusus GYLLENHAL, 1835 Published data: JAQUeT 1902b, FLeCK 1905, NeGRU and Roşca 1967, Raduta 2014

New data: Tulcea County: Pădurea Letea (1,5 m), 27.VII.2004, leg. GP, AM (Coll. Mus. R. ANGHELUȚǍ).

Larinus (Larinomesius) serratulae BECKER, 1864 Published data: Montandon 1887, Fleck 1905, Negru and RoşCa 1967, Raduta 2014

\section{*Larinus (Larinomesius) syriacus GYLLENHAL, 1835} (fig. 25)

New data: Tulcea County:Codru Monastery, 24-25. VI.2017, leg. KI, KJ, PA, TB; 2 km from Slava Rusă to NE, 3.VI.2018, leg. FK, KI, KJ, PA; Enisala (castle), 20.VI.2019, leg. KI, KJ, PA, TB; Constanţa County: Balta Limanu, 5-6.VII.2014, leg. KI, OA, PA, SzL; Gura Dobrogei, 25-27.VI.2017, leg. KI, KJ, PA, TB; Sinoie, Grindul Lupilor, 27.VI.2017, leg. KI, KJ, PA, TB; Murfatlar, Fântâniţa, 7-8.VI.2018, leg. KI.

\section{Larinus (Larinus) latus (HERBST, 1783)}

Published data: MonTANDON 1887; JAQUET 1899a; Fleck 1905; Negru 1957, 1968; Negru and RoşCa 1967; TeOdOR 1993; TeOdOR and MANOLE 1996; RADUTA 2014

New data: Tulcea County: Dinogeţia, 23.V.2002, leg. CŞ; Delta Dunării, Crişan, 26.VII.2003, leg. GP; Pădurea Babadag, 27.VII.2003, leg. CI; Babadag, 13.VIII.2003, 14.VIII.2003, leg. MM; M-ţii Măcin, Pricopan ( $150 \mathrm{~m}), 13$. VI.2004, leg. AR; Cheile Dobrogei (170-200 m), 14.VI.2004, leg. MM; M-ții Măcin, Pricopan (300 m), 21.VI.2004, 17.IV.2005, 
leg. AR; M-ţii Măcin, Valea lui Arsu, 6.VI.2005, leg. MC; M-ţii Măcin-Pricopan( 300-370 m), 16.VI.2005, leg. GP; Luncaviţa-liziera, 16.VI.2005, leg. CŞ; M-ţii Măcin, Îmbulzita Greci, 13.VI.2007, leg. CŞ; M-ţii Măcin, Dealurile Pricopanului, 14.VI.2007, leg. MC (Coll. Mus. R. Angheluță); Măcin, Igliţa, leg. ALM; Măcin, Greci, 11.VII.1960, leg. IS; Letea, 12.VII.1962, leg. MW; Pădurea Babadag, 20.VI.1963, leg. XS; C. A. Rosetti, 20.VII.1964, leg. XS; Măcin, 26.VI.1967, leg. MW; Pădurea Babadag, Slava Rusă, 26.VII.1993, leg. NG; Izvoarele, 21.V.1997, leg. RS; Izvoarele, 21.V.1997, leg. CP; Hagieni, 16.VIII.1983, leg. SzL; Enisala, 27.VI.1991, leg. NG; Revărsarea, 23.V.1997, leg. RS; Babadag, 6.VI.2011, leg. IŞI(Coll. Mus. G. AnTIPA) - Agighiol, 12.VI.1993, leg. TLA; Beştepe, 13.VI.1993, leg. TLA; Iancina, 11.VI.1993, leg. TLA; Braţul Sfântu Gheorghe, 23.VI.2003, leg. TB; Măcin (hillside, 200 m), 29.V.2005, leg. CsS; Codru Monastery, 23-25.V.2013, leg. KI, PA, RI, SzL; Enisala, 24-25.V.2013, leg. KI, PA, RI, SzL, 3031.III.2016, leg. FK, KI, PA, SzL; Enisala (castle), 21.V.2015, leg. PA, RI, SzK, SzL, 20.VI.2019, leg. KI, KJ, PA, TB; N from Nifon, 23.VI.2017, leg. KI, KJ, PA, TB; S from Hamcearca, 24.VI.2017, leg. KI, KJ, PA, TB; Turcoaia, $6 \mathrm{~km}$ from Cerna to NW, 3.VI.2018, leg. FK, KI, KJ, PA; M-ţii Măcin, Greci, 18-19.VI.2019, leg. KI, KJ, PA, TB; Constanţa County: Mangalia, leg. ALM; Valul lui Traian, 3.VI.1954, leg. XS; Pădurea Comorova, 11-12.IX.1959, leg. ŞN; Mangalia, Pădurea Hagieni, 5.VI.1962, leg. APG, Pădurea Hagieni, 6.VI.1965, leg. XS, 16.VII.1997, leg. AV; Hagieni, 04-08.VI.1964, leg. NS, 6.VI.1964, leg. ŞN, 28.VIII.1964, leg. NS, 6.VI.1965, leg. SSN, 6.VI.1965, leg. MW, 15.VI.1981, leg. NS, 17-19.VII.1997, leg. IoS; Băneasa, Canaraua Fetii, 16.VII.1964, leg. MW, 17-19.VII.1964, 30-31.V.1965, 2.VI.1965, leg. ŞN, 31.V.1965, leg. XS, 3.VII.1965, leg. MT, 11.VI.1967, leg. NS, 18.V.1993, 21.VII.1993, leg. CP, 1920.V.1993, leg. CH, 20.VII.1993, leg. GA, 19.V.1994, leg. CP, 19.V.1994, leg. MA, 26.VI.1995, leg. CP; Băneasa, 30.V.1965, leg. MW; Esechioi, 12-14. VI.1981, 17.VI.1981, leg. NS; Târguşor, 9.VII.1992, leg. RS; Limanu, Pădurea Hagieni, 10.VII.1992, leg. CP; Negureni, Pădurea Negureni, 18.V.1993, leg. CP; Furnica, Pădurea Dumbrăveni, 20.V.1994, leg. CP, 12.V.1995, 22.VI.1995, leg. MA, 13-14.V.1995, leg. GA; Canton Silvic Furnica, Pădurea Dumbrăveni, 13.V.1995, leg. CP; Mangalia Nord, Staţiunea Olimp, 19.VII.2003, leg. AP, 19-20.VII.2003, leg. GC, 19-20.VII.2003, leg. RS, 19.VII.2003, leg. MF; Tuzla, 21.VII.2003, leg. RS; Peştera, Ivrinezu Mic, 17.V.2015, leg. VG (Coll. Mus. G. AntiPA) - 3 km W of Crucea, Allah Bair (Nature Reserve), 1.V.2009, leg. JK; Gura Dobrogei, 28-29.IV.2012, leg. KI, 26.V.2013, leg. KI, PA, RI, SzL, 12.V.2017, leg. FK, KI, KJ, PA, 27.VI.2017, leg. KI, KJ, PA, TB; Istria, Cetatea Histria, 21.V.2015, leg. PA, RI, SzK, SzL, 26.VI.2017, leg. KI; Palazu Mic, 22.V.2015, leg. PA, RI, SzK, SzL; Cochirleni, 24.V.2015, leg. PA, RI,
SzK, SzL; Sinoie, Grindul Lupilor, 5.VI.2018, leg. FK, KI, KJ, PA; Vadu, Grindul Chituc, 6.VI.2018, leg. FK, KI, KJ, PA;

\section{Larinus (Larinus) pollinis LAICHARTING, 1781} (= brevis HeRBST, 1795)

New data: Tulcea County: Agighiol, 12.VI.1993, leg. TLA; Beştepe, 13.VI.1993, leg. TLA; Iancina, 11.VI.1993, leg. TLA.

\section{Larinus (Larinus) sibiricus GYLLENHAL, 1835 \\ (= aeruginosus HochHUTH, 1851) \\ Published data: JAQUET 1900a}

New data: Tulcea County: M-ţii Măcin,Valea lui Moş Enache, 05.X.2007, leg. CŞ; M-ţii Măcin, Chiscura Chelului (Parcul Naţional M-ţii Măcin, 295 m), 13.VI.2009, leg. CI (Coll. Mus. R. Angheluță) Babadag (forest), 23-25.V.2013, leg. KI, PA, RI, SzL; Codru Monastery, 23-25.V.2013, leg. KI, PA, RI, SzL; N from Nifon, 23.VI.2017, leg. KI, KJ, PA, TB; Turcoaia, $6 \mathrm{~km}$ from Cerna to NW, 24.VI.2017, leg. KI, KJ, PA, TB, 3.VI.2018, leg. FK, KI, KJ, PA; $1,5 \mathrm{~km}$ from Beidaud to SW, 25.VI.2017, leg. KI, KJ, PA, TB; Smârdan, Braţul Măcin, 2.VI.2018, leg. FK, KI, KJ, PA; Enisala (wooded pasture), 4.VI.2018, leg. FK, KI, KJ, PA; Enisala (castle), 20.VI.2019, leg. KI, KJ, PA, TB; Jurilovca, 23.VI.2019, leg. KI, KJ, PA, TB; Constanţa County: Hârşova, leg. ALM; Valul lui Traian, 19.VII.1962, leg. XS; Hagieni, 8.VI.1964, leg. NS; Agigea, 25.VI.1964, leg. IC (Coll. Mus. G. ANTIPA) - Oltina (village), 8.VII.2014, leg. KI, OA, PA, SzL; Palazu Mic, 22.V.2015, leg. PA, RI, SzK, SzL; Sinoie, Grindul Lupilor, 5.VI.2018, leg. FK, KI, KJ, PA, 22.VI.2019, leg. KI.

\section{Larinus (Larinus) vulpes (OLIVIER, 1807)}

New data: Constanţa County: Pădurea Hagieni, 20.VI.1964, leg. XS (Coll. Mus. G. ANTIPA) - Gura Dobrogei, 26.V.2013, leg. KI, PA, RI, SzL.

\section{Larinus (Phyllonomeus) carlinae (OLIVIER, 1807)} (= planus STEPHENS, 1831)

Published data: Negru and Roşca 1967, PerJu and PăLĂGeşiu 1986, Teodor and Manole 1996

New data: Tulcea County: Delta Dunării, Lacul Fortuna, 14.VI.2001, leg. CR (Coll. Mus. R. ANGHELUŢă) - M-ţii Măcin, to the Peak Ţuţuiatu, 28.IV.2010, leg. TLA; Codru Monastery, 1011.V.2017, leg. FK, KI, KJ, PA; Nifon, pârâul Pârlita valley, 23.VI.2017, leg. TB; Smârdan, Braţul Măcin, 7.V.2017, leg. KI, 2.VI.2018, leg. FK, KI, KJ, PA; 2 km from Slava Rusă to NE, 3.VI.2018, leg. FK, KI, KJ, PA; Constanţa County: Murfatlar, Fântâniţa, 7-8. VI.2018, leg. FK, KI, KJ, PA.

\section{Larinus (Phyllonomeus) centaurii (OLIVIER 1807)}

New data: Tulcea County: C. A. Rosetti, 14.VII.1962, leg. APG; Pădurea Letea, spre lacul Mesheiul Mic, 10.VI.1991, leg. DR; Jurilovca, Pădurea 6 Martie, 
10.V.1992, leg. NR; Delta Dunării, Haşmacul lui Omer, 18.VI.1993. IM (Coll. Mus. G. ANTIPA).

*Larinus (Phyllonomeus) filiformis PETRI, 1907 (fig. 26)

New data: Constanţa County: Balta Limanu, 5-6. VII.2014, leg. KI, OA, PA, SzL; Oltina (village), 8.VII.2014, leg. KI, OA, PA, SzL.

Larinus (Phyllonomeus) iaceae (FABRICIUS, 1775)

Published data: JaQuet 1899b, Fleck 1905, PerJu and PălăgeşiU 1986, TeOdor and Manole 1996, RADUTA 2014

New data: Tulcea County: M-ţii Măcin, Pricopan (370 m), 13.VI.2004, leg. MM; Cheile Dobrogei (170-200 m), 14.VI.2004, leg. GP; M-ţii Măcin, Pricopan (300370 m), 16.VI.2005, leg. GP; M-ţii Măcin, Dealurile Pricopanului, 14.VI.2007, leg. MC; M-ţii Măcin, Chiscura Chelului (Parcul Naţional M-ţii Măcin), 13.VI.2009, leg. CŞ (Coll. Mus. R. Angheluță); Pădurea Babadag, 20.VI.1963, leg. XS; Babadag, 6.VI.2011, leg. IŞ (Coll. Mus. G. Antipa)-M-ţii Măcin, Valea Fagilor (quarry), 30.V.2005, leg. CsS; Atmagea, 28.IV.2012, leg. KI; Codru Monastery, 23-25.V.2013, leg. KI, PA, RI, SzL, 10-11.V.2017, leg. FK, KI, KJ, PA, 24-25.VI.2017, leg. KI, KJ, PA, TB, 4.VI.2018, leg. FK, KI, KJ, PA; Enisala, 24-25.V.2013, leg. KI, PA, RI, SzL; Enisala (castle), 21.V.2015, leg. PA, RI, SzK, SzL; Nifon, pârâul Pârlita valley, 23.VI.2017, leg. KI, KJ, PA, TB; N from Nifon, 23.VI.2017, leg. KI, KJ, PA, TB; Turcoaia, $6 \mathrm{~km}$ from Cerna to NW, 24.VI.2017, leg. KI, KJ, PA, TB, 3.VI.2018, leg. FK, KI, KJ, PA; 1,5 km from Beidaud to SW, 25.VI.2017, leg. KI, KJ, PA, TB; Enisala, Cetatea Heraclea, 25.VI.2017, leg. KI, KJ, PA, TB; Smârdan, Braţul Măcin, 28.VI.2017, leg. KI, KJ, PA, TB; 3 km from Slava Rusă to NE, 24.VI.2017, leg. KI, KJ, PA, TB; 2 km from Slava Rusă to NE, 3.VI.2018, leg. FK, KI, KJ, PA; Enisala (wooded pasture), 4.VI.2018, leg. FK, KI, KJ, PA, 20.VI.2019, leg. KI, KJ, PA, TB; M-ţii Măcin, Greci, 18-19.VI.2019, leg. KI, KJ, PA, TB; Constanţa County: Mangalia, Pădurea Hagieni, 4.VI.1962, leg. APG; Comorova, 26.VI.1963, leg. XS; Mangalia, Hagieni, 30.VII.1963, leg. ŞN; Agigea, 20.VI.1964, leg. IC; Hagieni, 8.VI.1964, leg. NS, 6.VI.1965, leg. MW, 12.VII.1992, leg. IM; Băneasa, Canaraua Fetii, 20.V.1994, leg. GA, 26.VI.1995, leg. CP; Furnica, Pădurea Dumbrăveni, 15.V.1995, leg. GA, 22.VI.1995, leg. MA; Rezervaţia Naturală Agigea, 17.VII.2003, leg. MF; Mangalia Nord, Staţiunea Olimp, 19.VII.2003, leg. MF (Coll. Mus. G. Antipa) - Gura Dobrogei, 26.V.2013, leg. KI, PA, RI, SzL, 25-27.VI.2017, leg. KI, KJ, PA, TB, 7.VI.2018, leg. FK, KI, KJ, PA; Murfatlar, Fântâniţa, 4-5.VII.2014, leg. KI, OA, PA, SzL, 7-8.VI.2018, leg. FK, KI, KJ, PA; Balta Limanu, 5-6.VII.2014, leg. KI, OA, PA, SzL; Esechioi (forest) 7.VII.2014, leg. KI, OA, PA, SzL; Băneasa, Canaraua Fetii, 7.VII.2014, leg. KI, OA, PA, SzL, 23.V.2015, leg. PA, RI, SzK, SzL;

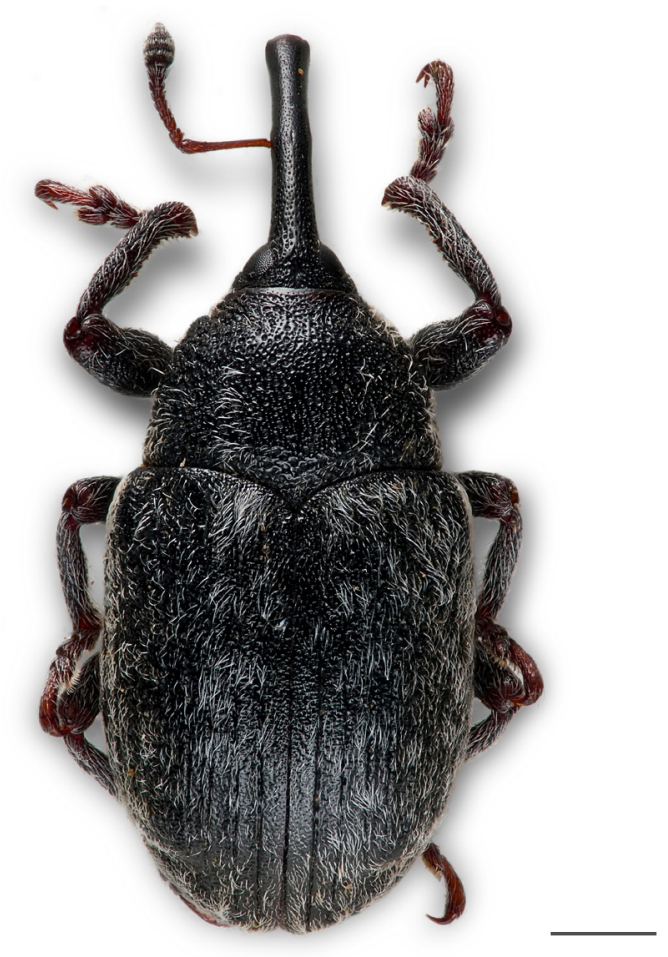

Fig. 26. Larinus (Phyllonomeus) filiformis PeTRI, 1907. Scale bar: $1 \mathrm{~mm}$. Photo Zoltán CSATA

Istria, Cetatea Histria, 21.V.2015, leg. PA, RI, SzK, SzL, 26-28.VI.2017, leg. KI, KJ, PA, TB, 6.VI.2018, leg. FK, KI, KJ, PA; Palazu Mic, 22.V.2015, leg. PA, RI, SzK, SzL; Sinoie, Grindul Lupilor, 27.VI.2017, leg. KI, KJ, PA, TB, 5.VI.2018, leg. FK, KI, KJ, PA, 22.VI.2019, leg. KI, KJ, PA, TB; Vadu, Grindul Chituc, 22.VI.2019, leg. KI, KJ, PA, TB.

\section{Larinus (Phyllonomeus) ovaliformis CAPIOMONT, 1874}

Published data: JAQUET 1899a

Larinus (Phyllonomeus) planus (FABRICIUS, 1792) (= rusticanus GYLlenHal, 1935)

Published data: RADUTA 2014

New data: Tulcea County: Delta Dunării, Lacul Fortuna, 14.VI.2001, leg. CR.

Larinus (Phyllonomeus) sturnus (SCHALLER, 1783) Published data: JAQUeT 1899b, Fleck 1905, NegRU and RoşCa 1967, Teodor and Manole 1996, Raduta 2014

New data: Tulcea County: Pădurea Letea, 27.VII.2004, leg. CŞ (Coll. Mus. R. Angheluță); Caraorman, 6.VII.1968, leg. MW; Maliuc, Mila 26, 8.VIII.1991, leg. IM; Babadag, 6.VI.2011, leg. IŞI (Coll. Mus. G. ANTIPA) - Beştepe, 13.VI.1993, leg.TLA; Canalul Magearu, 16-17.VI.1993, leg. TLA; Gorgova, 16.VI.1993, leg. TLA; Braţul Sfântu Gheorghe, 23.VI.2003, leg. TB; Codru Monastery, 23-25.V.2013, leg. KI, PA, RI, SzL; Enisala, 24-25.V.2013, leg. KI, PA, RI, SzL; Smârdan, Braţul Măcin, 28.VI.2017, leg. KI, KJ, PA, TB; Enisala (wooded pasture), 4.VI.2018, 
leg. FK, KI, KJ, PA; 2 km from Slava Rusă to NE, 3.VI.2018, leg. FK, KI, KJ, PA; Constanţa County: Băneasa, Canaraua Fetii, 12.VI.1962, 26.VI.1995, leg. CP (Coll. Mus. G. AnTIPA) - Gura Dobrogei, 28-29. IV.2012, leg. KI, 30.IV-2.V.2014, leg. KI, IZ, PA, SzL; Murfatlar, Fântâniţa, 4-5.VII.2014, leg. KI, OA, PA, SzL; Istria, Cetatea Histria, 26-28.VI.2017, leg. KI, KJ, PA, TB.

\section{Larinus (Phyllonomeus) turbinatus GYLLENHAL,} 1835

Published data: Fleck 1905, Teodor and Manole 1996, RADUTA 2014

New data: Tulcea County: Pădurea Babadag, 27.VII.2003, leg. CI; Mircea Vodă, 29.VII.2003, leg. CŞ; Slava Rusă (200 m), 13.VI.2004, leg. GP; M-ţii Măcin, Valea lui Arsu, 6.VI.2005, leg. MC; M-ţii Măcin, Plopişor (300-370 m), 15.VI.2005, leg. MC; M-ții Măcin, Pricopan, (300-370 m), 16.VI.2005, leg. GP; M-ţii Măcin, Vf. Țuţuiatu (400 m), 26.V.2006, leg. MC; M-ţii Măcin, Nifon, 12.VI.2007, leg. CŞ (Coll. Mus. R. Angheluță); C. A. Rosetti, VII.1950, leg. IC; Pădurea Letea, 16.VII.1962, leg. MW; Maliuc, Mila 26, 7-8.VIII.1991, leg. IM, 09.08.1991, leg. RS; Pădurea Caraorman, 19.V.1992, leg. CP; Sfântu Gheorghe, Grindul Sărăturile, 13.VI.1995, leg. MiS; Teliţa, Mănăstirea Celic Dere, 20.V.1997, leg. RS; Luncaviţa, Valea Fagilor, 25.V.2005, leg. CB; Slava Rusă, Pădurea Babadag, 19.VII.2006, leg. IM; Zaghen, 16.V.2013, leg. IŞI (Coll. Mus. G. ANTIPA) - Braţul Sfântu Gheorghe, 21-22.VI.2003, leg. TB; Caraorman, Canal Litcov, 12.V.2007, leg. NJ, RE, SoL, VJ; Enisala, 24-25.V.2013, leg. KI, PA, RI, SzL; Doloşman, 11.05.1992, leg. TLA; Canalul Magearu, 16-17.VI.1993, leg. TLA; Iancina, 11.VI.1993, leg. TLA; Periprava, 1.VII.2005, leg. DDC; Babadag (forest), 2.VI.2005, leg. CsS; 3 km from Slava Rusă to NE, 24.VI.2017, leg. KI; Smârdan, Braţul Măcin, 7.V.2017, leg. KI, 2.VI.2018, leg. FK, KI, KJ, PA; Greci (village), 8.V.2017, leg. KI; $2 \mathrm{~km}$ from Slava Rusă to NE, 3.VI.2018, leg. FK, KI, KJ, PA; Constanţa County: Băneasa, Canaraua Fetii, 20.V.1993, leg. AP; Rezervaţia Agigea, 18.VII.2003, leg. RS; Mangalia Nord, Staţiunea Olimp, 20.VII.2003, leg. AA (Coll. Mus. G. ANTIPA) - Murfatlar, Fântâniţa, 22.V.2015, leg. PA, RI, SzK, SzL.

Lixus (Broconius) rubicundus ZOUBKOFF, 1833 Published data: Fleck 1905, Negru and RoşCa 1967, IENişTEA 1968, TeOdor and MANOLE 1996, Raduta 2014

New data: Tulcea County: Enisala, 24-25.V.2013, leg. KI, PA, RI, SzL; Smârdan, Braţul Măcin, 28.VI.2017, leg. TB; Constanţa County: Delta Dunării, G. Babarada-Şontea, 30.VII.2004, leg. GP (Coll. Mus. R. Angheluță) - Murfatlar, Fântâniţa, 22.V.2015, leg. PA, RI, SzK, SzL; Băneasa, Canaraua Fetii, 23.V.2015, leg. PA, RI, SzK, SzL; 2 km from Sinoie to SE, 27.VI.2017, leg. KI, KJ, PA, TB; Istria,
Cetatea Histria, 21.VI.2019, leg. KI, KJ, PA, TB.

Lixus (Compsolixus) albomarginatus BOHEMAN, 1842

Published data: Jaquet 1899b; Fleck 1905; Teodor 1993; Teodor \& Manole 1996; Raduta 2014

New data: Tulcea County: M-ţii Măcin, Vf. Dogaru, 29.08.2007, leg. CI; M-ţii Măcin, Drumul Vinului, 30.VIII.2007, leg. CI (Coll. Mus. R. Angheluță); Constanţa County: Valul lui Traian, 1957, leg. XS (Coll. Mus. G. Antipa) - Vadu env., Grindul Chituc, 21.IX.2010, leg. JK; Murfatlar, Fântâniţa, 7-8. VI.2018, leg. FK, KI, KJ, PA.

\section{Lixus (Compsolixus) ascanii (LINNÉ, 1767)}

Published data: Montandon 1887, 1908; FleCK 1905; Negru and RoşCa 1967; Raduta 2014

\section{Lixus (Compsolixus) ochraceus BOHEMAN, 1842}

New data: Tulcea County: Măcin, leg. ALM; Somova, 28.VII.1987, leg. IM; Celic Dere, 20.V.1997, leg. CH (Coll. Mus. G. ANTIPA) - M-ţii Măcin, Valea Fagilor (quarry), 30.V.2005, leg. CsS; Atmagea env., 24.IX.2010, leg. JK; $1.5 \mathrm{~km} \mathrm{~N}$ of Horia, Lacul Horia (lake), 24.IX.2010, leg. JK; Babadag (forest), 13.IX.2016, leg. KI, KJ, PA, TB; Smârdan, Braţul Măcin, 22.VI.2017, leg. TB; Jijila, 19.VI.2019, leg. KI, KJ, PA, TB; Constanţa County: Grădina env., 19.IX.2010, leg. JK; Gura Dobrogei, 28-29.IV.2012, leg. KI; Murfatlar, Fântâniţa, 4-5.VII.2014, leg. KI, OA, PA, SzL, 22.V.2015, leg. PA, RI, SzK, SzL, 2425.IX.2015, leg. KI, KJ, PA, 14.IX.2016. leg. KI, KJ, PA, TB, 7-8.VI.2018, leg. FK, KI, KJ, PA; Ostrov, Lacul Bugeac, 16.IX.2016, leg. KI, KJ, PA, TB.

\section{Lixus (Dilixellus) bardanae (FABRICIUS, 1787)}

New data: Tulcea County: Periprava, 2.VII.1963, leg. XS (Coll. Mus. G. AnTIPA) - Atmagea env., 24.IX.2010, leg. JK; 2 km from Slava Rusă to NE, 3.VI.2018, leg. KI; Constanţa County: Canalul Magearu, 16-17.VI.1993, leg. TLA; Băneasa, Canaraua Fetii (quarry), 2-3.IV.2016, leg. FK, KI, PA, SzL.

\section{Lixus (Dilixellus) fasciculatus BoHEMAN, 1835}

New data: Tulcea County: Delta Dunării, C. Magearu-D. Veche, 26.VII.2004, leg. GP (Coll. Mus. R. ANGHELuȚă); Sfântu Gheorghe, Delta, 7.VII.1983, leg. NS (Coll. Mus. G. ANTIPA) - Nifon, pârâul Pârlita valley, 23.VI.2017, leg. KI, KJ, PA, TB.

Lixus (Dilixellus) linearis OLIVIER, 1807

(= cribricollis BOHEMAN, 1835)

Published data: JAQUeT 1902b, FleCK 1905

New data: Constanţa County: Dumbrăveni, 23.V.1965, leg. NS (Coll. Mus. G. ANTIPA).

Lixus (Dilixellus) pulverulentus (SCOPOLI, 1763) (= algirus FABRICIUS, 1801; = angustatus FABRICIUS, 
1775)

New data: Tulcea County: Pădurea Babadag, 12.V.1982; Celic Dere, 20-24.V.1994, leg. CP; Izvoarele, lacul Horia, 21.V.1997, leg. RS; Revărsarea, 23.V.1997, leg. CP (Coll. Mus. G. ANTIPA) - Babadag (forest), 23-25.V.2013, leg. KI， PA， RI， SzL, 30.IV.2014, leg. KI, IZ, PA, SzL; Codru Monastery 23-25.V.2013, leg. KI, PA, RI, SzL, 10-11.V.2017, leg. FK, KI, KJ, PA; Enisala, 24-25.V.2013, leg. KI, PA, RI, SzL; Slava Rusă, 24-25.V.2013, leg. KI, PA, RI, SzL; Constanţa County: Esechioi, 26.V.1962, leg. NS; Băneasa, Canaraua Fetii, 18.V.1993, leg. AP, 18.V.1993, leg. CP; Canton Silvic Furnica, Pădurea Dumbrăveni, 13.V.1995, leg. CP; Furnica, Rezervaţia Dumbrăveni, 13-14.V.1995, leg. CP, 14.V.1995, leg. GA; Mangalia Nord, Staţiunea Olimp, 20.VII.2003, leg. RS (Coll. Mus. G. AnTIPA) - Gura Dobrogei, 28-29.IV.2012, leg. KI, 30.IV-2.V.2014, leg. KI, IZ, PA, SzL, 12.V.2017, leg. FK, KI, KJ, PA; Murfatlar, Fântâniţa，4-5.VII.2014， leg. KI，OA，PA，SzL, 22.V.2015, leg. PA, RI, SzK, SzL, 24-25.IX.2015, leg. KI, KJ, PA; Băneasa, Canaraua Fetii (quarry), 7.VII.2014, leg. KI, OA, PA, SzL; Cochirleni, 24.V.2015, leg. PA, RI, SzK, SzL; Ostrov, Lacul Bugeac, 16.IX.2016, leg. KI, KJ, PA, TB; Pecineaga (forest), 14.V.2017, leg. FK, KI, KJ, PA.

\section{Lixus (Dilixellus) punctiventris BOHEMAN, 1835}

New data: Constanţa County: Cochirleni, 8.VII.2014, leg. KI, OA, PA, SzL.

\section{Lixus (Epimeces) cardui OLIVIER, 1807}

Published data: Fleck 1905, Montandon 1908, TeOdor 1993, TeOdor and Manole 1996, Raduta 2014

New data: Tulcea County: Dinogeţia, 23.V.2002, leg. CŞ; Cheile Dobrogei (170-200 m), 14.VI.2004, leg. MM; M-ţii Măcin, Luncaviţa (200 m), 16.VI.2005, leg. MC; Izvoarele Măcinului (300-370 m), 16.VI.2005, leg. MC (Coll. Mus. R. Angheluță); Periprava, Pădurea Letea, 24.VI.1966, leg. ŞN; Sulina, 12.VII.1967, leg. AtR; Ciucurova, 18-19.VI.1981, leg. NS; Insula Popina, Lacul Razelm, 8.V.1992, leg. NG; Capul Dolojman, Lacul Razelm, 11.V.1992, leg. NG; Maliuc, Mila 26, 18-19.V.1992, leg. IM; M-ții Măcin, Dealurile Pricopanului, 14.VI.2007, leg. MC; Izvoarele, 21.V.1997, leg. RS; Celic Dere, 24.V.1997, leg. RS (Coll. Mus. G. ANTIPA) -Agighiol, 12.VI.1993, leg. TLA; Beştepe, 14.VI.1993, leg. TLA; Canalul Magearu, 17.VI.1993, leg. TLA; Iancina, 11.VI.1993, leg. TLA; Caraorman, Canal Litcov, 12.V.2007, leg. NJ, RE, SoL, VJ; Enisala, 24-25.V.2013, leg. KI, PA, RI, SzL, 29.IV-3.V.2014, leg. KI, IZ, PA, SzL; Enisala (castle), 21.V.2015, leg. PA, RI, SzK, SzL, 20.VI.2019, leg. KI, KJ, PA, TB; Smârdan, Braţul Măcin, 7.V.2017, leg. FK, KI, KJ, PA, 2.VI.2018, leg. FK, KI, KJ, PA; 23 August, 17.IX.2016, leg. FK, KI, KJ, PA; S from Hamcearca, 24.VI.2017, leg. KI; Enisala (wooded pasture), 4.VI.2018, leg. FK, KI,
KJ, PA; Constanţa County: Agigea, 15.VI.1994, leg. XS; Băneasa, 30.V.1965, leg. MW; Băneasa, Canaraua Fetii, 31.V.1965, leg. XS; Hagieni, 6.VI.1965, leg. ŞN, 15.VI.1981, leg. NS; Pădurea Hagieni, 6.VI.1965, leg. XS; Târguşor, 8.VII.1992, leg. RS; Limanu, Pădurea Hagieni, 11.VII.1992, leg. RS (Coll. Mus. G. ANTIPA) - Gura Dobrogei, 28-29.IV.2012, leg. KI, 26.V.2013, leg. KI, PA, RI, SzL, 30.IV-2.V.2014, leg. KI, IZ, PA, SzL, 12.V.2017, leg. FK, KI, KJ, PA, 25-27.VI.2017, leg. KI; Istria, Cetatea Histria, 21.V.2015, leg. PA, RI, SzK, SzL, 11.V.2017, leg. FK, KI, KJ, PA; Cochirleni, 24.V.2015, leg. PA, RI, SzK, SzL; Sinoie, Grindul Lupilor, 5.VI.2018, leg. FK, KI, KJ, PA.

\section{Lixus (Epimeces) filiformis (FABRICIUS, 1781)}

(= elongatus GoEzE, 1777)

Published data: JAQUet 1902b, Fleck 1905, Negru and RoşCa 1967, Raduta 2014

New data: Tulcea County: M-ţii Măcin, Greci (400 m), 13.VI.2004, leg. GP; M-ţii Măcin, Chiscura Chelului (Parcul Naţional M-ţii Măcin, 295 m), 13.VI.2009, leg. CI (Coll. Mus. R. Angheluţă); Izvoarele, 21.V.1997, leg. RS; Babadag, 6.VI.2011, leg. IŞI (Coll. Mus. G. Antipa) - Doloşman, 11.V.1992, leg. TLA; Insula Popina, 9.V.1992, leg. TLA; M-ţii Măcin, Valea Fagilor (quarry) 30.V.2005, leg. CsS; Babadag (forest), 2005.VI.2, leg. CsS; Atmagea, 28.IV.2012, leg. KI; Codru Monastery, 23-25.V.2013, leg. KI, PA, RI, SzL, 19.V.2015, leg. PA, RI, SzK, SzL, 10-11.V.2017, leg. FK, KI, KJ, PA; Enisala, 2425.V.2013, leg. KI, PA, RI, SzL, 29.IV-3.V.2014, leg. KI, IZ, PA, SzL; Slava Rusă, 24-25.V.2013, leg. KI, PA, RI, SzL, 20.V.2015, leg. PA, RI, SzK, SzL; Enisala (castle), 21.V.2015, leg. PA, RI, SzK, SzL, 10.V.2017, leg. FK, KI, KJ, PA, 20.VI.2019, leg. KI, KJ, PA, TB; M-ţii Măcin, 5 km S Greci, 8.V.2017, leg. FK, KI, KJ, PA; Nifon, pârâul Pârlita valley, 23.VI.2017, leg. KI, KJ, PA, TB; N from Nifon, 23.VI.2017, leg. KI, KJ, PA, TB; Turcoaia, $6 \mathrm{~km}$ from Cerna to NW, 24.VI.2017, leg. KI, KJ, PA, TB, 19.VI.2019, leg. KI, KJ, PA, TB; S from Hamcearca, 24.VI.2017, leg. KI, KJ, PA, TB; 1,5 km from Beidaud to SW, 25.VI.2017, leg. KI, KJ, PA, TB; Smârdan, Braţul Măcin, 22.VI.2017, leg. KI, 18.VI.2019, leg. KI; Enisala (wooded pasture), 4.VI.2018, leg. FK, KI, KJ, PA; M-ţii Măcin, Greci, 18-19.VI.2019, leg. KI, KJ, PA, TB; Constanţa County: Pădurea Hagieni, 19.VI.1962, leg. AtR, 6.VI.1965, leg. ŞN; Palas, 21.VI.1962, leg. AKI; Băneasa, Canaraua Fetii, 1.V.1965, leg. XS; Dumbrăveni, 23.V.1965, leg. NS; Furnica, Pădurea Dumbrăveni, 14.V.1995, leg. GA, 15.V.1996, leg. MA; Rezervaţia Naturală Agigea, 17.VII.2003, leg. MF; (Coll. Mus. G. AnTIPA) - Gura Dobrogei, 28-29. IV.2012, leg. KI, 26.V.2013, leg. KI, PA, RI, SzL, 30.IV-2.V.2014, leg. KI, IZ, PA, SzL, 7.VI.2018, leg. FK, KI, KJ, PA; Cheile Dobrogei, 2.V.2014, leg. KI, IZ, PA, SzL; Esechioi (forest), 7.VII.2014, leg. KI, OA, PA, SzL; Istria, Cetatea Histria, 21.V.2015, leg. PA, RI, SzK, SzL, 26-28.VI.2017, leg. KI, KJ, PA, 
TB; Palazu Mic, 22.V.2015, leg. PA, RI, SzK, SzL; Murfatlar, Fântâniţa 22.V.2015, leg. PA, RI, SzK, SzL; Băneasa, Canaraua Fetii, 23.V.2015, leg. PA, RI, SzK, SzL; Negureni, Pădurea Valea Cişmelelor, 23.V.2015, leg. PA, RI, SzK, SzL; Cochirleni, 24.V.2015, leg. PA, RI, SzK, SzL; Hagieni, Pădurea Hagieni (forest), 17.IX.2016, leg. FK, KI, KJ, PA; 23 August, 17.IX.2016, leg. FK, KI, KJ, PA; 2 km from Sinoie to SE, 27.VI.2017, leg. KI; Sinoie, Grindul Lupilor, 5.VI.2018, leg. FK, KI, KJ, PA; Crucea, 9.VI.2018, leg. FK, KI, KJ, PA.

*Lixus (Epimeces) scolopax BoHEMAN, 1835 New data: Constanţa County: Murfatlar, Fântâniţa, 7-8.VI.2018, leg. FK, KI, KJ, PA.

Lixus (Eulixus) desertorum GEBLER, 1830

Published data: JAQUET 1899b, FleCK 1905

Lixus (Eulixus) iridis OLIVIER, 1807

Published data: (Jaquet 1899a, FleCK 1905, Negru and RoşCa 1967, TeOdor and Manole 1996, RAduta 2014

New data: Tulcea County: Delta Dunării, Caraorman, 13.VI.2001, leg. CR (Coll. Mus. R. Angheluță); Celic Dere, 20-24.V.1994, leg. CP, 24.V.1997, leg. RS; Valea Teilor, 22.V.1997, leg. CH (Coll. Mus. G. ANTIPA) - M-ţii Măcin, Valea Fagilor (dried lake bed) 30.V.2005, leg. CsS; Lacul Roşu (near the Red Lake), 6.VII.2005, leg. DDC; Greci (village), 8.V.2017, leg. FK, KI, KJ, PA; Nifon, pârâul Pârlita valley, 23.VI.2017, leg. KI, KJ, PA, TB; Smârdan, Braţul Măcin, 22.VI.2017, leg. KI, KJ, PA, TB; M-ţii Măcin, Greci, 18-19.VI.2019, leg. KI, KJ, PA, TB; Enisala, 20.VI.2019, leg. KI; Constanţa County: Dumbrăveni, 1.V.1956., 23.V.1965, leg. NS; Mangalia, Pădurea Hagieni, 6.VI.1963, leg. ID; Negureni, Pădurea Negureni, 18.V.1993, leg. CP; Băneasa, Canaraua Fetii, 18.V.1993, leg. AP; Furnica, Pădurea Dumbrăveni, 14.V.1995, leg. GA; Negureni, 21.VI.1996, leg. CP (Coll. Mus. G. ANTIPA) - Gura Dobrogei, 28-29.IV.2012, leg. KI, 26.V.2013, leg. KI, PA, RI, SzL, 7.VI.2018, leg. FK, KI, KJ, PA; Băneasa, Canaraua Fetii, 23.V.2015, leg. PA, RI, SzK, SzL; Cochirleni, 24.V.2015, leg. PA, RI, SzK, SzL; Ostrov, Lacul Bugeac, 16.IX.2016, leg. KI, KJ, PA, TB; Esechioi, Lacul Bugeac, towards the south Lake Bugeac, 16.IX.2016, leg. KI, KJ, PA, TB.

\section{Lixus (Eulixus) punctirostris BOHEMAN, 1842}

New data: Tulcea County: M-ţii Măcin, Pricopan, 1.VI.2005, leg. CsS; M-ţii Măcin, Greci env., 6.V.2009, leg. JK; Smârdan, Braţul Măcin, 22.VI.2017, leg. KI, KJ, PA, TB, 2.VI.2018, leg. KI.

\section{Lixus (Lixus) paraplecticus LINNAEUS, 1758}

New data: Tulcea County: Sălcieni, Delta, 22.VII.1950, leg. IC (Coll. Mus. G. ANTIPA) Caraorman, 17.V.1991, leg. AR; Smârdan, Braţul
Măcin, 22.VI.2017, leg. KI, KJ, PA, TB.

Lixus (Ortholixus) angustus (HERBST, 1795)

New data: Tulcea County: M-ţii Măcin, Valea lui Smalena, 12.VI.2007, leg. MC (Coll. Mus. R. Angheluță); Constanţa County: Istria, Cetatea Histria, 14.IX.2016, leg. KI, KJ, PA, TB.

\section{Lixus (Ortholixus) bituberculatus SMRECZYŃSKI, 1968}

Published data: Trnka, Stejskal and Skuhrovec 2016

New data: Tulcea County: M-ţii Măcin, Valea Fagilor (dried lake bed), 30.V.2005, leg. CsS; Constanţa County: Hagieni, 4.VI.1964, leg. ŞN; Furnica, Rezervaţia Dumbrăveni, 13-14.V.1995, leg. CP (Coll. Mus. G. ANTIPA) - Hagieni, Pădurea Hagieni (forest), 17.IX.2016, leg. KI; Gura Dobrogei, 12.V.2017, leg. KI.

Lixus (Ortholixus) cinerascens SCHÖNHERR, 1832

(= elegantulus BoHEMAN, 1843)

Published data: Montandon 1887, Fleck 1905

New data: Tulcea County: Murighiol, 9.IX.2007, leg. CI; M-ţii Măcin,Valea lui Moş Kalâm, 5.X.2007, leg. CŞ, CI; M-ţii Măcin, Valea lui Moş Enache, 5.X.2007, leg. CŞ, CI; M-ţii Măcin, Valea lui Arsu, 5.X.2007, leg. CŞ (Coll. Mus. R. Angheluță); Periprava, Delta, 26.VII.1957, leg. IS (Coll. Mus. G. Antipa) Turcoaia, $6 \mathrm{~km}$ from Cerna to NW, 3.VI.2018, leg. FK, KI, KJ, PA, 19.VI.2019, leg. KI, KJ, PA, TB; Constanţa County: Agigea, 18.VI.1962, leg. AtR, 10.VIII.1962, leg. ID; Pădurea Hagieni, 16.VII.1997, leg. AV, 19.VII.1997, leg. CP (Coll. Mus. G. ANTIPA) - Grădina env., 19.IX.2010, leg. JK; Esechioi (forest), 7.VII.2014, leg. KI, OA, PA, SzL; Ostrov, Lacul Bugeac, northward Lake Bugeac, 16.IX.2016, leg. KI, KJ, PA, TB.

\section{Lixus (Ortholixus) vilis (Rossi, 1790)}

Published data: JAQUET 1899b, FLECK 1905

New data: Tulcea County: M-ţii Măcin, Chiscura Chelului (Parcul Naţional M-ţii Măcin, 295 m), 13.VI.2009, leg. CI (Coll. Mus. R. ANGHeluŢă).

\section{Lixus (Phillixus) incanescens BOHEMAN, 1835}

Published data: JAQUeT 1899b, FleCK 1905

New data: Tulcea County: Delta Dunării, G. Babarada-Şontea, 30.VII.2004, leg. GP; Murighiol, 9.IX.2007, leg. CI (Coll. Mus. R. Angheluţă) - Enisala, 29.IV-3.V.2014, leg. KI, IZ, PA, SzL; Sălcioara, 13.IX.2016, leg. KI, KJ, PA, TB; Smârdan, Braţul Măcin, 18.VI.2019, leg. KI; Constanţa County: Furnica, Pădurea Dumbrăveni, 13-14.V.1995, leg. CP (Coll. Mus. G. AnTIPA) - Istria env., Cetatea Histria, 2-4.V.2009, 19.IX.2010, leg. JK; Grădina env., 19.IX.2010, leg. JK.

Lixus (Phillixus) scabricollis BOHEMAN, 1843 
Lixus (Phillixus) subtilis BOHEMAN, 1835

New data: Tulcea County: Delta Dunării, G. Babarada-Şontea, 30.VII.2004, leg. GP (Coll. Mus. R. ANGHELuȚǍ); Maliuc, Mila 26, 8.VIII.1991, leg. IM; Greci, 6.IX.2005, leg. CB (Coll. Mus. G. ANTIPA) - Sulina, 4.VII.2005, leg. DDC; Constanţa County: Comorova, 31.VIII.1963, leg. AtR; Negureni, Pădurea Negureni, 22.VII.1993, leg. GA; Băneasa, Canaraua Fetii, 19.V.1994, leg. GA, 26.V.1995, leg. CP; Furnica, Rezervaţia Dumbrăveni, 13-14.V.1995., 22.VI.1995, leg. CP; Negureni, 21.VI.1996, leg. CP (Coll. Mus. G. ANTIPA) $-3 \mathrm{~km}$ W of Crucea, Allah Bair (Nature Reserve), 1.V.2009, leg. JK; Esechioi (forest), 7.VII.2014, leg. KI, OA, PA, SzL; Băneasa, Canaraua Fetii (forest), 23.V.2015, leg. PA, RI, SzK, SzL, 15-17.IX.2016, leg. KI, KJ, PA, TB, 20.VI.2017, leg. CS; Esechioi, 15.IX.2016, leg. KI, KJ, PA, TB.

Microlarinus cf. lypriformis (Wollaston, 1861)

New data: Constanţa County: $9 \mathrm{~km}$ E of Istria, Cetatea Histria, 4-5.VI.2010, leg.RŠ; 26-28.VI.2010, leg. FP.

\section{Rhinocyllus conicus (FRÖLICH, 1792)}

Published data: JAQUET 1899b, FLECK 1905, Montandon 1908

New data: Tulcea County: Codru Monastery, 23-25.V.2013, leg. KI, PA, RI, SzL; Enisala, 24 25.V.2013, leg. KI, PA, RI, SzL; N from Nifon, 23.VI.2017, leg. KI, KJ, PA, TB; Turcoaia, $6 \mathrm{~km}$ from Cerna to NW, 24.VI.2017, leg. KI, KJ, PA, TB, 3.VI.2018, leg. KI; M-ţii Măcin, Greci, 18-19. VI.2019, leg. KI; Constanţa County: Gura Dobrogei, 26.V.2013, leg. KI, PA, RI, SzL.

\section{[Rhinocyllus oblongus CAPIOMONT, 1873]}

Published data: JAQUET 1899b, FLECK 1905 - Spread: Southern Europe, Northern Africa and Asia Minor: Turkey and Israel. Incorrect identification.

MESOPTILIINAE LACORDAIRE, 1863

Magdalidini PASCOE, 1870

Magdalis (Edo) ruficornis (LINNÉ, 1758)

New data: Tulcea County: - Celic Dere, 20.V.1997, leg. CH (Coll. Mus. G. ANTIPA) - Slava Rusă, 1-3.V.2014, leg. KI, IZ, PA, SzL; M-ții Măcin, to the Peak Ţuţuiatu, 28.IV.2010, leg. TLA.

Magdalis (Magdalis) frontalis GYLLENHAL, 1827 New data: Constanţa County: Hagieni, 22.V.1993, leg. $\mathrm{CH}$ (Coll. Mus. G. ANTIPA).

\section{Magdalis (Magdalis) rufa GERMAR, 1823}

Published data: TeOdor and Manole 1996

New data: Constanţa County: Limanu, 10.VII.1992, leg. RS (Coll. Mus. G. ANTIPA) - Murfatlar, Fântâniţa, 4-5.VII.2014, leg. KI, OA, PA, SzL; Balta Limanu, 5-6.VII.2014, leg. KI, OA, PA, Sz; Crucea, 9.VI.2018,
Magdalis (Odontomagdalis) armigera (GEOFFROY, 1785)

New data: Tulcea County: Caraorman, 14-15.V.1992, leg. TLA; Enisala, 10.V.2017, leg. FK, KI, KJ, PA; M-ţii Măcin, Nifon (forest), 23.VI.2017, leg. KI, KJ, PA, TB; 2 km from Slava Rusă to NE, 3.VI.2018, leg. FK, KI, KJ, PA; Constanţa County: Gura Dobrogei, 28-29.IV.2012, leg. KI.

Magdalis (Porrothus) cerasi (LINNÉ, 1758)

New data: Tulcea County: Slava Rusă, 11.V.2017, leg. KI.

MOLYTINAE SCHÖNHERR, 1823

Cryptorhynchini SCHÖNHERR, 1825

Tylodina LACORDAIRE, 1866

Acalles (Acalles) echinatus (GERMAR, 1823)

New data: Tulcea County: Slava Rusă, 4.V.2009, leg. JK, 1-3.V.2014, leg. KI, IZ, PA, SzL; NE of Topolog, 4.V.2009, leg. JK.

Acalles (Acalles) papei A. SOLARI \& F. SOLARI, 1905 New data: Tulcea County: Babadag (forest), 3.VI.2018, leg. FK, KI, KJ, PA.

\section{Acallocrates colonnellii BAHR, 2003}

New data: Tulcea County: Celic Dere, 22-24.V.1997, leg. RS, CH (Coll. Mus. G. ANTIPA) - Babadag (forest), 22.IX.2015, leg. KI, KJ, PA; $2 \mathrm{~km}$ from Slava Rusă to NE, 3.VI.2018, leg. FK, KI, KJ, PA.

Gasterocercini ZHERIKHIN, 1991

Gasterocercus depressirostris (FABRICIUS, 1792)

Published data: NEGRU 1968

New data: Tulcea County: M-ţii Măcin, Greci, 18-19. VI.2019, leg. KI.

Lepyrini W. KIRBY, 1837

Lepyrus capucinus (SCHALLER, 1783)

New data: Tulcea County: Delta Dunării, Grindul Letea, Haşmacul lui Omer, 10.VI.1991, leg. DR; Celic Dere, 24.V.1997, leg. RS (Coll. Mus. G. ANTIPA) - Gorgova, 16.VI.1993, leg. TLA; Pădurea Letea (forest), 17.VI.1993, leg. TLA; Babadag (forest), 30.IV.2014, leg. KI, IZ, PA, SzL; Smârdan, Braţul Măcin, 22.VI.2017, leg. KI, 2.VI.2018, leg. FK, KI, KJ, PA; Constanţa County: Băneasa, Canaraua Fetii, 19.V.1993, leg. CH (Coll. Mus. G. ANTIPA).

Lepyrus palustris palustris (SCOPOLI, 1763)

Published data: Negru 1968

New data: Tulcea County: Ostrovul Haralambie, 26.VI.2008, leg. MS (Coll. Mus. G. ANTIPA).

Molytini SCHÖNHERR, 1823

Hylobiina W. KIRBY, 1837

Hylobius (Callirus) abietis (LINNÉ, 1758) 
New data: Tulcea County: Sulina, 09.VII.1967, leg. APG (Coll. Mus. G. AnTIPA); Constanţa County: Valul lui Traian, 12.VII.1957, leg. XS (Coll. Mus. G. ANTIPA).

Hylobius (Callirus) transversovittatus (GoEzE, 1777) Published data: Montandon 1908, Negru 1968

New data: Tulcea County: Smârdan, Piatra Fetei, 18.VII.2003, leg. CŞ (Coll. Mus. R. ANGHELuță); Chilia Veche, 27.VI.1963, leg. XS; Delta, Periprava, 5-8.XI.1964, leg. NS; Delta Dunării, Pădurea Caraorman, 24.VI.1991, leg. PG; Caraorman, 26.VI.1991, leg. IP; Celic Dere, 20-24.V.1994, leg. CP (Coll. Mus. G. AnTIPA) - Periprava, 1.VII.2005, leg. DDC.

Molytina SCHÖNHERR, 1823

Liparus (Trysibius) tenebriodes (PALLAS, 1781)

Published data: JAQUeT 1900a, Negru and RoşCa 1967, RADUTA 2014

New data: Tulcea County: Măcin, leg. ALM; Babadag, 23.VI.1955, leg. NS; Codru, 1.VI.1970, leg. NS (Coll. Mus. G. AntIPA).

Plinthina LACORDAIRE, 1863

Minyops senaci DesBrochers des Loges, 1895

Published data: FLECK 1905, Negru and RoşCA 1967, Osella and Bello 2010, Raduta 2014

New data: Tulcea County: W of Horia, 6.V.2009, leg. JK.

Pissodini Gistel, 1848

Pissodina Gistel, 1848

Pissodes (Pissodes) castaneus (DEGEER, 1775)

New data: Constanţa County: Murfatlar, Fântâniţa, 22.V.2015, leg. PA, RI, SzK, SzL.

Trachodini Gistel, 1848

Trachodes (Trachodes) hispidus (LINNÉ, 1758)

Published data: Montandon 1887, Fleck 1905

New data: Tulcea County: Nifon, pârâul Pârlita valley, 23.VI.2017, leg. KI.

Typoderini Voss, 1965

Anchonidium (Anchonidium) ulcerosum (AUBÉ, 1850)

Published data: Montandon 1908

New data: Tulcea County: M-ţii Măcin, 2 km NW Nifon, pârâul Curături, 27.VI.2015, leg. MGy; M-ţii Măcin, Nifon (forest), 23.VI.2017, leg. KI, KJ, PA, TB.

SCOLYTINAE LATREILLE, 1804

Cryphalini LindeMANn, 1876

Ernoporicus caucasicus (LINDEMANN, 1876)

(=Euptilius caucasicus Lindemann, 1876)

Published data: RADUTA 2014

Ernoporus tiliae (PANZER, 1793)
Published data: RADUTA 2014

New data: Tulcea County: Slava Rusă, 1-3.V.2014, leg. KI, IZ, PA, SzL.

Trypophloeus granulatus (RATZEBURG, 1837)

Published data: RADUTA 2014

Dryocoetini Lindemann, 1876

Xylocleptes bispinus (DUFTSCHMID, 1825)

Published data: RADUTA 2014

New data: Tulcea County: Slava Rusă, 1-3.V.2014, leg. KI, IZ, PA, SzL; Codru Monastery, 30-31. III.2016, leg. FK, KI, PA, SzL; Constanţa County: Gura Dobrogei, 30.IV-2.V.2014, leg. KI, IZ, PA, SzL.

Hylesinini ERICHSON, 1836

Hylesinus crenatus (FABRICIUS, 1787)

Published data: RADUTA 2014

Hylesinus taranio (DANTHOINE, 1788) (= oleiperda FABRicius, 1792)

Published data: RADUTA 2014

Hylesinus varius (FABRICIUS, 1775) (= Leperisinus fraxini (PANZER 1779))

Published data: RADUTA 2014

Pteleobius kraatzii (ЕICHHOFF, 1864)

Published data: RADUTA 2014

Hypoborini NÜsSLIN, 1911

Liparthrum arnoldi SEMENOV, 1902

Published data: RADUTA 2014

Ipini BEDEL, 1888

Pityogenes conjunctus ReITTER, 1887

New data: Tulcea County: Codru Monastery, 30-31. III.2016, leg. FK, KI, PA, SzL.

Pityogenes trepanatus (NöRDLINGER, 1848)

New data: Constanţa County: Crucea, 9.VI.2018, leg. FK, KI, KJ, PA.

Phloeotribini Chapuis, 1869

Phloeotribus muricatus (EGGERs, 1929)

(= Phloeophthorus muricatus EGGERS, 1929)

Published data: RADUTA 2014

New data: Tulcea County: Codru Monastery, 19.V.2015, leg. PA, RI, SzK, SzL; M-ţii Măcin, 5 km S Greci, 8.V.2017, leg. KI; Constanţa County: Gura Dobrogei, 26.V.2013, leg. KI, PA, RI, SzL.

Polygraphini CHAPUIS, 1869

Carphoborus perrisi (CHAPUIS, 1869)

Published data: RADUTA 2014

Scolytini Latreille, 1804

Scolytus intricatus (RATZEBURG, 1837)

Published data: RADUTA 2014 
Scolytus kirschii kirschii SKALITZKY, 1876

Published data: RADUTA 2014

Scolytus multistriatus (MARSHAM, 1802)

Published data: RADUTA 2014

Scolytus pygmaeus (FABRICIUS, 1787)

Published data: RADUTA 2014

Scolytus rugulosus (P. W. J. MÜLLER, 1818)

Published data: RADUTA 2014

New data: Tulcea County: Mahmudia, 20.X.1970, leg. ŞN (Coll. Mus. G. ANTIPA); Constanţa County: Pădurea Hagieni (forest), 6.VII.2014, leg. KI, OA, PA, SzL.

Scolytus scolytus (FABRICIUS, 1775)

Published data: RADUTA 2014

Xyleborini LeConte, 1876

Xyleborus dryographus (RATZEBURG, 1837)

Published data: RADUTA 2014

Xyleborus monographus (FABRICIUS, 1792)

Published data: RADUTA 2014

Xyleborus xylographus (SAY, 1826)

Published data: RADUTA 2014

\section{Discussion}

A number of 56 species with very old or old reports were either not found by us or are present in the studied collections. The latest ones were collected between 1950-1977. For these weevils, new research is needed in the locations where they have been reported in the past to see if these species still exist in the area or are extinct.

In the Cooperative Catalogue of Palaearctic Coleoptera Curculionoidea (Alonso-ZaraZaGa et al. 2017) for the species Graptus triguttatus (FABRICIUS) is not specified that it lives in Romania, although it has many older and newer reports from various regions of Romania (TeOdor and ANTonie Vlad 2007). So G. triguttatus (FABRICIUS) is not new to Romania's fauna. Through our reports we confirm its presence in Romania.

The species reported in the past by other authors: Aulacobaris andalusiaca (BRIs.), Aulacobaris prasina (BoH.), Peritelus sphaeroides Germ., Pseudomeira rudis (BoH.), Asproparthenis lusca (Chevrolat), Pseudocleonus (Pseudocleonus) carinatus (GYL.) and Rhinocyllus oblongus CAPIOMONT do not exist in the researched area nor in Romania. They were wrongly identified by the authors of the respective reports. Likewise, Otiorhynchus (Prilisvanus) opulentus GERM. does not exist in the researched area. It lives only in the Carpathians.
In recent publications (ALONSO-ZARAZAGA et al. 2017) Eusomus beckeri TOURNIER is considered synonymous of Eusomus ovulum GERM.. We still present them here separately because there are clear morphological differences between the individuals of these two species (fig. 22). We consider that their taxonomic status should be analyzed again.

For names of the species from the genera Rhinoncus and Stomodes, in addition to the information in Cooperative Catalogue of Palaearctic Coleoptera Curculionoidea (Alonso-Zarazaga et al. 2017) we also used data on the revision of Rhinoncus pericarpius (L.) species group (HUANG and CoLONNELLI 2014) and the revision of in the Stomodes tolutarius Schoenherr species group (BoroveC and HLAvíč 2018).

These 724 species of Curculionoidea from the Danube Delta and Dobruja represent 55\% of the total species of Curculionoidea reported so far in Romania, which highlights the specific richness of this area.

The reporting of 50 new species for the Romanian fauna and of the two new species for science show that this area deserves to be further studied from the point of view of the Curculionoidea group.

\section{Acknowledgements}

The authors bring their warmest thanks for the help of the unknown reviewer who contributed through the corrections, remarks and suggestions made, to a significant improvement in the content and quality of the original version of the manuscript. We thank dr. Melanya STAN (Muzeul Naţional de Istorie Naturală „Grigore Antipa” Bucureşti), Gabriela PATriche (Complexul Muzeal de Ştiinţele Naturii "Răsvan Angheluţă" Galaţi) and (†) dr. MerkL Ottó (Magyar Természettudományi Múzeum Budapest) for providing access to their museums' collections. We also thank Tallósi Béla, FetYKó Kinga, Orosz András, RozNer István, SzÉKelY Kálmán, Csősz Sándor, dr. MaKRANCZY György, NÉDLI Judit, E. ReCueroGIL, Somar László and Vörös Judit for making their collecting material available for us. We thank SzÉKeLY Levente and Kocs János for their unselfish help, making our collecting trips successful. We also thank dr. HANGAY György for the English translation of the results of our work. We thank dr. CsATA Zoltán, dr. NÉMETH Tamás and dr. GYöRGY Zoltán for taking photos of weevils and agreeing us to use these photos in our work. We also thank SzÖKE Viktória for illustration of fig. 22.

\section{References}

Alonso-Zarazaga M.A. (1989) Revision of the supraspecific taxa in the Palaearctic Apionidae SCHOENHERR, 1823. 1. Introduction and subfamily Nanophyinae SEIDLITZ, $1891 \quad$ (Coleoptera, Curculionoidea). Fragmenta Entomologica, Roma, 21(2): 205-262.

Alonso-Zarazaga M.A. (1990) Revision of the supraspecific taxa in the Palaearctic Apionidae Schoenherr, 1823 (Coleoptera, Curculionoidea). 2. Subfamily Apioninae, ScHOENHERR, 1823: Introduction keys and descriptions. Graellsia 46: 19-156. 
Alonso-Zarazaga M.A., Barrios H., Borovec R., Bouchard P., Caldara R., Colonnelli E., Gültekin L., Hlaváč P., Korotyaev B., Lyal C.H.C., Machado A., Meregalli M., Pierotti H., Ren L., SÁnChez-Ruiz M., Sforzi A., Silfverberg H., Skuhrovec J., TrÝzna M., Velázquez de Castro A.J. and Yunakov N.N. (2017) Cooperative Catalogue of Palaearctic Coleoptera Curculionoidea. Monografias electrónicas S.E.A. 8: $1-729$.

Altea T. and Osella G. (1991) Caratteristiche del popolamento a Coleotteri Curculionoidea della Riserva Naturale Orientata "Valle Orfento" (Majella-Abruzzo). Studi Trentini di Scienze Naturali, Acta Biologica, Terento 68: 239-266.

Angelov P. (1976) Coleoptera, Curculionidae I, (Apioninae, Otiorhynchinae). Fauna na Bulgaria, 5, Bulgarian Academy of Sciences, Sofia (in Bulgarian).

BIALOOKI Z.P. (2007) On new taxa of Entiminae (Coleoptera: Curculionidae) from Turkey and SouthEastern Europe. Annals of the Upper Silesian Museum (Entomology) 14-15: 135-195.

Borovec R. (1992) Revision of Lacordairius CH. BRISOUT, 1866, a subgenus of Trachyphloeus GERMAR, 1817 (Coleoptera, Curculionidae). EOS-Revista Espanola de Entomologia 68(2): 147-158.

Borovec R. and Pelletier J. (2009) Revision of the genus Foucartia JACQUELIN DU VAL, 1855 (Coleoptera: Curculionidae: Entiminae: Sciaphilini). Acta Societatis Zoologicae Bohemicae 73: 1-26.

Borovec R. and Hlavéč P. (2018) Review of the Stomodes tolutarius species group (Coleoptera: Curculionidae: Entiminae: Otiorhynchini) from the Balkan Peninsula with description of two new species from Greece. Zootaxa 4497(1): 111-126.

Caldara R. and Podlussány A. (2018) New faunistic data on Palaearctic species of the tribes Mecinini and Tychiini (Coleoptera: Curculionidae, Curculoninae). Folia entomologica hungarica 79: 51-53.

Ciochia V. (1985) La structure et la dynamique de l'entomofaune phytophage et entomophage des agrobiocénoses a betterave sucrière de Roumanie. Travaux du Museum National d'Histoire Naturelle “Grigore Antipa" 27: 193-213.

Colonnelli E. and Whitehead D.R. (1990) Palaearctic thistle weevils of the genus Bangasternus GozIS (Coleoptera, Curculionidae). Fragmenta Entomologica, Roma 22(1): 185-217.

DiECKMANN L. (1963) Die palaearktischen Arten der Untergattung Pseudorchestes BEDEL aus der Gattung Rhynchaenus CLAIRY (Coleoptera, Curculionidae). Entomologische Abhandlungen des Staatlichen Museums für Tierkunde, Dresden 29(2): 275-327.

DrĂGHIA I. 1972. Insectes Mineurs (Insecte minere), L'entomofaune du Grind de Caraorman, Delta du Danube. Travaux du Museum National d'Histoire Naturelle "Grigore Antipa” 12: 221-228.

ENDRŐDI S. (1960) Ormányosbogarak II [Snoutbeetles II], Curculionidae II. Fauna Hungariae 10(5), Akadémiai Kiado, Budapest (in Hungarian).

ENDRöDI S. (1961) Ormányosbogarak I [Snoutbeetles I], Curculionidae I. Fauna Hungariae 10 (4), Académiai Kiadó, Budapest (in Hungarian).

ENDRŐDI S. (1963) Ormányosbogarak III [Snoutbeetles III], Curculionidae III. Fauna Hungariae 10 (6), Académiai
Kiadó, Budapest (in Hungarian).

ENDRöDI S. (1968) Ormányosbogarak IV [Snoutbeetles IV], Curculionidae IV. Fauna Hungariae 10 (7), Académiai Kiadó, Budapest (in Hungarian).

ENDRŐDI S. (1971) Ormányosbogarak V [Snoutbeetles V], Curculionidae V. Fauna Hungariae 10 (8), Académiai Kiadó, Budapest (in Hungarian).

Fleck E. (1905) Die Coleopteren Rumäniens. Bulletin de la Société des Sciences de Bucarest 14(5): 491-570.

FleCK E. (1906) Die Coleopteren Rumäniens. Bulletin de la Société des Sciences de Bucarest 3-4: 3-336.

Holecova M. (1993) Bagous bagdatensis PIC 1904 (= Bagous wagneri DieCKMANN 1964) (Curculionidae, Coleoptera) a little known palaearctic weevil. Entomological Problems, Bratislava 24(1): 73-80.

Huang J. and Colonnelli E. (2014) On the true identity of Curculio pericarpius Linnaeus, 1758 (Coleoptera: Curculionidae). Fragmenta entomologica 46(1-2): 117120.

HuRmuZaCHI C. (1904) Troisième cataloque des coléoptères récoltès par membres de la Société des Naturalistes de Roumanie et déterminé par Const. Hurmuzachi (Cernăuţi). Bulletin de la Société des Sciences de Bucarest 13(1, 2): 52-65.

IEnişTeA M.A. (1968) Ord. Coleoptera, "L'entomofaune de l'île de Letea (Delta du Danube)". Travaux du Museum National d'Histoire Naturelle "Grigore Antipa" 9: 97-114.

IENIŞTEA M.A. (1974) Contributions à la connaisance des Coléoptères du Delta du Danube (Le grind Caraorman). Travaux du Museum National d'Histoire Naturelle "Grigore Antipa” 14: 239-249.

IENIŞTEA M.A. and NeGRU Ş. (1956) Specii de coleoptere noi pentru fauna R.P.R. [New beetle species for fauna R.P.R.] Comunicările Academiei R.P.R. 6(8): 995-997 (in Romanian).

Ionescu M., Mitru L. and ŢurCA I. (1963) Contribuţii la cunoaşterea biologiei şi combaterii gărgăriţei Tychius flavus BEKER, un nou dăunător al culturilor de lucernă din R.P.R. [Contributions to the knowledge of the biology and control of the weevil Tychius flavus BEKER, a new pest of alfalfa crops from R.P.R.] Analele Secţiei de Protecţia Plantelor, Bucureşti 1: 153-162 (in Romanian).

Ionescu M., Tomoroga M. and ȚurCA I. (1967) Noi cercetări asupra bio-ecologiei şi combaterii gărgăriţei lucernei (Tychius flavus BECKER) [New research on bioecology and the control of the alfalfa weevil (Tychius flavus BeKer)]. Analele Secţiei de Protecţia Plantelor, Bucureşti 4: 271-182 (in Romanian).

JAQUET M. (1898a) Insectes récoltés par M. JAQUET en 1897, et déterminés par Mr. Ernest PoNCY, assistant au Musée d'histoire naturale de Genéve. Bulletin de la Société des Sciences de Bucarest 7(1): 52-56.

JAQUET M. (1898b) Faune de la Roumanie. Insectes récoltés par Mr. le Dr. M, JAQUET et déterminés par Mr. E. PONCY, entomologiste à Genéve. Bulletin de la Société des Sciences de Bucarest 7(2): 185-187.

JAQUET M. (1898c) Faune de la Roumanie. Coléoptères récoltés par M. JAQUET en 1897, et déterminés par Mr. E. Poncy, entomologiste à Geneve. Bulletin de la Société des Sciences de Bucarest 7(6): 493-495.

JAQUET M. (1899a) Curculionides récoltés par Mr. le Dr. JAQUET en 1898 et déterminés par Mr. le Dr. STIERLIN 
de Schaffhouse. Bulletin de la Société des Sciences de Bucarest 8(1, 2): 124-126.

JAQUET M. (1899b) Faune de la Roumanie. Curculionides déterminés par Monsieur Le Docteur G. STIERLIN de SchaffHouse. Bulletin de la Société des Sciences de Bucarest 8(4, 5): 366-368.

JAQuet M. (1900a) Faune de la Roumanie. Coleopterés récoltés par Mr. le Dr. Jaquet et detérminés par Mr. E. Poncy à Genève, Bulletin de la Société des Sciences de Bucarest, 9(2, 3): 392-400.

JAQUET M. (1900b) Faune de la Roumanie. Coléoptères récoltés en 1899 par Mr. Le Dr. M, JAQUET et déterminés par Mr. Le Dr. G. STIERlin A'SchaffHOuSE. Bulletin de la Société des Sciences de Bucarest 9(6): 758-760.

JAQUET M. (1901) Faune de la Roumanie. Insectes récoltés par Mr. Le Dr. M, JAQUET en 1899 et déterminés par Mr. le Dr. E. PonCY, entomologiste à Genève. Bulletin de la Société des Sciences de Bucarest 10(5): 483-497.

JAQUeT M. (1902a) Faune de la Roumanie. Coléoptères récoltés en 1899 par Mr. Le Dr. M, JAQUET et déterminés par Mr. Le Dr. G. STIERLin A'SchafFHOUSE. Bulletin de la Société des Sciences de Bucarest 11(1, 2): 314-316.

JAQUeT M. (1902b) Faune de la Roumanie. Coléoptères récoltés en 1900 par Mr. Le Dr. M. JAQUET et déterminés par Mr. Le Dr. G. STIERLin A'SchaffHOUSE. Bulletin de la Société des Sciences de Bucarest 11(1, 2): 606-608.

JAQUET M. (1904) Faune de la Roumanie. Coléopterès récoltés par M. JAQUET et déterminés par M. E. PONCY entomologiste à Genève. Bulletin de la Société des Sciences de Bucarest 13(1-2): 66-69.

KizUB I. and SlutSKy I.A. (2018) New data on the distribution of Pseudaplemonus artemisiae (F. MorawITZ, 1861) (Coleoptera: Apionidae) in Ukraine and the Palearctic Region. Munis Entomology and Zoology 13(1): 98-105.

Kodada J. and Holecova M. (1994) Biologie und Verbreitung der Gattung Dicranthus MoTsCHULSKY, 1845 (Coleoptera, Curculionidae). Verhandlungen des 14 Internaţionalen Symposium für Entomologistik in Mitteleuropa (SIEEC), München: 282-285.

LENGYEL P. http://regithink.transindex.ro

Magnano L. and Osella G. (1971) Revizione del genere Parameira SeIDlitz (Coleoptera, Curculionidae) e descrizione di una nuava specie di Crimea. Memorie del Museo Civico di Storia Naturale di Verona 19: 237-266.

Montandon A.L. (1887) Excursions en Dobroudja. Bulletin de la Société d'Études scientifiques d'Angers 16: 31-64.

Montandon A.L. (1906) Notes sur la faune entomologique de la Roumanie Bulletin de la Société des Sciences de Bucarest 15: 30-80.

MontAndon A.L. (1908) Notes sur la faune entomologique de la Roumanie. Additions au Catalogue des Coléoptéres. Bulletin de la Société des Sciences de Bucarest 17(1,2): 67-122.

NeGRU Ş. (1957) Contribuţiune la cunoaşterea faunei coleopterologice a Mangaliei şi împrejurimilor ei (Nota I). [Contribution to the knowledge of the coleopterological fauna of Mangalia and its surroundings (Note I)] Analele Universităţii C. I. Parhon Bucureşti, Seria Ştiinţele Naturii 16: 117-130 (in Romanian).

Negru Ş. (1968) Ord. Coleoptera, "L'entomofaune de l'île de Letea (Delta du Danube)". Travaux du Museum National d'Histoire Naturelle "Grigore Antipa" 9: 81-95.
Negru Ş. and RoşCA A. (1967) Ord Coleoptera, "L'entomofaune des forêts du sud de la Dobroudja". Travaux du Museum National d'Histoire Naturelle "Grigore Antipa" 7: 138-141.

Osella G. (1973) Descritione di quatro nuove specie di Rhytirhinini d'Anatolia ed una di Dobrugia, e ridescrizione di Horridorhinus phrygius (Coleoptera, Curculionidae). Fragmenta Entomologica, Roma 9(3): 157-178.

Osella G. and Bello C. (2010) Revisione di Minyops Schoenherr, 1823 e Paraminyops nov. gen. (Coleoptera, Curculionidae, Molytinae). Memorie del Museo Civico di Storia Naturale di Verona (II serie), Sezione Scenzie delle vita 19: 5-136.

Osella G. and Di Marco C. (1993) Nuovi Curculionoidea della fauna Siciliana (Coleoptera). Bollettino del Museo civico di Storia Naturale di Verona 20: 345-370.

Osella G. and Magnano L. (1984) I coleotteri attelabidi e Curculionidi a diffusione transadriatica. Lavori della Società italiana di Biogeografia, nuova serie 10: 701792.

PANIN S. (1951) Determinatorul coleopterelor dăunătoare şi folositoare din Republica Populară Română. [The identification guide of harmful and useful beetles in the Romanian People's Republic] Editura de Stat pentru Literatură Ştiințifică şi Didactică, Bucureşti (in Romanian).

PerJu T. and PăLĂGeşıU I. (1986) Cercetări de biologie şi ecologie asupra unor specii de Curculionidae seminifage. [Biological and ecological research on some species of seminiferous Curculionidae] Lucrările celei de a III-a Conferinţe de Entomologie, Iaşi, 20-22 mai, 1983: 469475 (in Romanian).

Perju T. and Teodor L.A. (1998) Agenţi potenţiali de combatere biologică a unor buruieni-problemă din agroecosisteme. [Potential biological control agents for problem weeds in agroecosystems] Lucrările Ştiinţifice ale celei de a IV-a Conferinţe Naţionale Pentru Protecţia Mediului, Braşov: 131-138 (in Romanian).

Procheş Ş. (1999) Five weevil species (Coleoptera: Curculionidae) new for the Romanian fauna. Travaux du Museum National d'Histoire Naturelle "Grigore Antipa” 41: 325-330.

RADutA M. (2014) Biodiversitatea Dobrogei [Dobruja Biodiversity] http://www.scribd.com/doc/245200399/ Biodiversitatea-Dobrogei\#scribd (in Romanian)

SCHUBERT F.T.A. (1956) Ein bemerkenswertes Ergebnis einer mazedonischen Apionenausbeute. Apion breiti WGN.: Richtigstellung und Vervollständigung der Beschreibung. Fragmenta Balcanica Musei Macedonici Scientiarum Naturalium 1: 243-246.

SkuHrovec J. (2009) Digital-Weevil-Determination for Curculionoidea of West Palaearctic. Transalpina: Hypera / Limobius / Metadonus (Hyperinae: Hyperini). SNUDEBILLER, Studies on taxonomy, biology and ecology of Curculionoidea, CURCULIO-Institute, Mönchengladbach 10: 39-47.

Skuhrovec J., Gosik R. and Stejskal R. (2010) DigitalWeevil-Determination for Curculionoidea of the West Palearctic: Transalpina: Brachypera/Donus (Hyperinae: Hyperini). SNUDEBILLER. Studies on taxonomy, biology and ecology of Curculionoidea, CURCULIOInstitute, Mönchengladbach 11: 102-108.

Skuhrovec J., Stejskal R., Trnka F. and Gosik 
R. (2014) Digital-Weevil-Determination for Curculionoidea of West Palaearctic. Cleonini (Lixinae). Snudebiller: Studies on taxonomy, biology and ecology of Curculionoidea, CURCULIO-Institute, Mönchengladbach 15 (227): 1-18.

StüBen P.E., Bayer Ch., Sprick P. and Behne L. (2011) Digital-Weevil-Determination for Curculionoidea of the West Palearctic: Transalpina: Polydrusus (Entiminae: Polydrusini). SNUDEBILLER. Studies on taxonomy, biology and ecology of Curculionoidea, CURCULIOInstitute, Mönchengladbach 12: 12-24.

StüBen P.E., Müller G., KRátKÝ J., BaYer Ch., Behne L. and Sprick P. (2013) Digital-WeevilDetermination for Curculionoidea of the West Palearctic: Transalpina: Ceutorhynchinae (2. part). (Ceuthorhynchini: Amalorrhynchus, Drupenatus Poophagus, Coeliodes, Pseudocoeliodes, Coeliodinus, Eucoeliodes, Neoxyonyx, Thamiocolus, Micrelus, Zacladus, Phrydiuchus, Stenocarus, Nedyus, Ceutorhynchus:Marklissus). SNUDEBILLER: Studies on taxonomy, biology and ecology of Curculionoidea, CURCULIO-Institute, Mönchengladbach 14: 1-24.

StüBen P.E., Müller G., Müller, U., KrátKÝ J., Bayer Ch., Behne L. and Sprick P. (2014) DigitalWeevil-Determination for Curculionoidea of the West Palearctic: Transalpina: Ceutorhynchinae (3. part). (Ceutorhynchini: Datonychus, Ethelcus, Glocianus, Hadroplontus, Microplontus, Mogulones, Mogulonoides, Neoglocianus, Oprohinus, Parethelcus, Prisistus, Ranunculiphilus). SNUDEBILLER: Studies on taxonomy, biology and ecology of Curculionoidea, CURCULIO-Institute, Mönchengladbach 15: 1-25.

StÜBen P.E., Bayer CH., Bahr F., Sprick P. and BehNe L. (2015) Digital-Weevil-Determination for Curculionoidea of the West Palearctic: Transalpina: Phyllobiini. SNUDEBILLER. Studies on taxonomy, biology and ecology of Curculionoidea, CURCULIOInstitute, Mönchengladbach 16: 1-13.

TEODOR L.A. (1993) Contribuţii la cunoaşterea curculionidelor (Coleoptera) din Delta Dunării.
[Contributions to the knowledge of weevils (Coleoptera) from the Danube Delta] Analele Ştiințifice ale Institutului Delta Dunării, Tulcea 2: 193-196 (in Romanian).

Teodor L.A. and Manole T. (1996) Noi contribuţii la cunoaşterea curculionidelor din Rezervaţia Biosferei "Delta Dunării" (Coleoptera: Curculionidae). [New contributions to the knowledge of weevils from the "Danube Delta" Biosphere Reserve (Coleoptera: Curculionidae] Buletin de informare. Societatea lepidopterologică română 7(3, 4): 255-260 (in Romanian).

Teodor L.A. and Antonie Vlad I. (2007) Suprafamilia Curculionoidea [Superfamily Curculionoidea], pp.148168. In: Moldovan O.T., Cîmpean M., Borda D., IEPURE S. and ILIE V. (eds.), Lista faunistică a României (specii terestre şi de apă dulce) [The faunistic list of Romania (terrestrial and freshwater species)]. Casa Cărţii de Ştiinţă, Cluj-Napoca, Romania (in Romanian).

Trnka F., Stejskal R. and Skuhrovec J. (2016) The morphology of the immature stages of two rare Lixus species (Coleoptera, Curculionidae, Lixinae) and notes on their biology. ZooKeys 604(2): 87-116.

YUNAKov N.N. and NADEIN S.K. (2006) New taxa of geophilic Entiminae (Coleoptera: Curculionidae) from the Balkan Peninsula. Caucasus and Central Asia, Entomologische Abhandlungen 63(1, 2): 77-98.

WaGNeR H. (1910) Beitrag zur entomologischen Fauna Rumäniens. Die Arten der Gattung Apion HBsT. (Col.), vorzugsweise gesammelt von Herrn A. L. MonTANDON. Bulletin de la Société des Sciences de Bucarest 19: 939-947.

WANAT M. (1992) The review of Metapion gaudiale- and oculare species groups (Coleoptera: Apionidae). Genus, International Journal of Invertebrate Taxonomy 3(1): 39-61.

Wanat M. (1995) Systematics and phylogeny of the tribe Ceratapiini (Coleoptera: Curculionoidea. Apionidae). Genus, International Journal of Invertebrate Taxonomy, Supplement: 1-406.

https://hu.wikipedia.org/wiki/Dobrudzsa.
Irén Kocs

Székely National Museum,

Kós Károly 10,

Sfântu Gheorghe, RO-520055, Romania

E-mail: kocsiren.sznm@gmail.com
Jiří KRÁTKÝ

Třebechovická 821, CZ-50003

Hradec Králové, Czech Republic

E-mail:macshort@tiscali.cz

Received: 15.11 .2021

Accepted: 30.11.2021

Published online: 31.12 .2021

Article number: ER25202105

doi: 10.24193/entomolrom.25.5
Attila PodLussány

Budapest, Hungary

E-mail: podlussany.1941@gmail.com
Lucian Alexandru TEODOR

Dent of Taxonomy and Ecology

Cluj-Napoca, RO-400006, Romania

E-mail: lucianteodor@yahoo.com,

teodor.lucian@ubbcluj.ro 